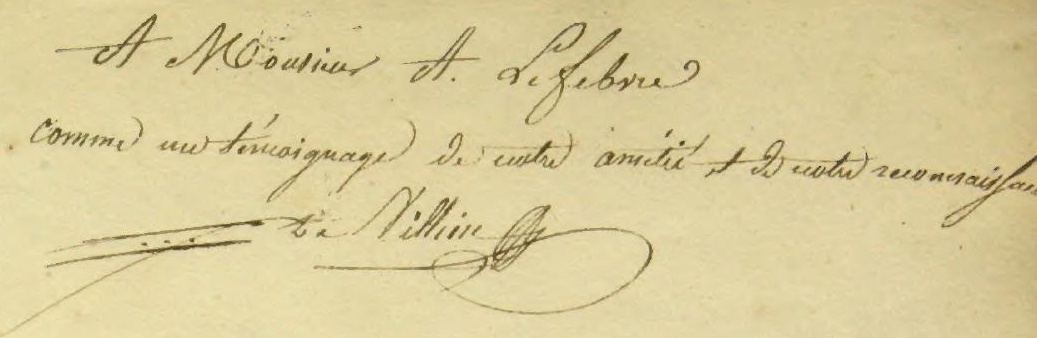

TABLEAUX SYNOPTIQUES

DES

\title{
LÉPIDOPTÈRES D'EUROPE.
}




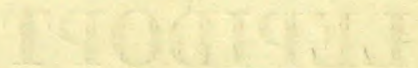

Paris. - Imprimerie de PLASSAN, rue de Vaugirard, $\mathrm{n}^{2}$ a. 


\section{TABLEAUX SYNOPTIQUES}

DES

\section{LÉPIDOPTÈRES D'EUROPE,}

CONTENAXT

\section{LA DESGRIPTION DE TOUS LES LÉPIDOPTÉRES}

CONNUS JUSQU'A GE JOUR,

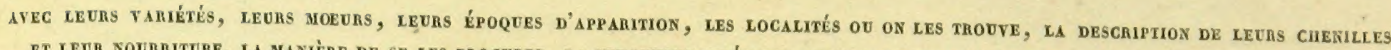

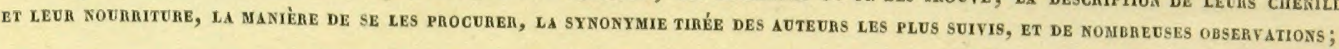

\section{PAR MM. DE VILLIERS ET GUENÉE,}

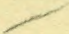
...... Nullum esse librum tam malum ut nón ex aliqua parte prodesset.

Puxk lancien

TOME PREMIER.

\section{DIURNES.}

\section{PARIS.}

MÉQUIGNON-MARVIS PÈRE ET FILS, LIBRAIRES,

RUE DU JARDINET, $N^{\circ} 15$.

A LONDRES, - CHEZ J.-B. BAILLIÈRE, 219 REGENT-STREET,

1835 . 
QL

$$
\begin{aligned}
& 555 \\
& \text { A1 }
\end{aligned}
$$

v75

$+$

की

Entomalogy

c.3882

8.10 

Mettre l'Entomologie à la portée de toutes les bourses et de toutes les intelligences, tel est le but de cet ouvrage. Le plan n'est pas
neuf, mais l'exécution est encore à trouver.

Quant à la forme de ce species, nous renonçons volontiers au mérite de la priorité. Peut-être cependant pourrions-nous la revendiquer avec quelque fondement, puisque l'un de nous s'occupait d'une œuvre à peu près semblable à celle-ci, et avait déjà presque terminé la famille des Diurnes à l'époque où M. Godart entreprit son Histoire naturelle des Lépidoptères, et que ce fut l'amitié qui l'unissait à ce naturaliste recommandable qui l'engagea à discontinuer son travail; mais notre prétention a moins été de faire un ouvrage neuf qu'un ouvrage utile. Analyser ce que les auteurs tant anciens que modernes ont écrit sur cette matière, offrir aux jeunes entomologistes un tableau exact et complet du nombre, de l'habitat et des mœurs des Lépidoptères d'Europe sous leurs trois états, avec une description qui, sans être trop courte, n'exige qu'un coup d'œil pour faire reconnaitre l'objet; suppléer d'une manière commode et élégante aux catalogues manuscrits qui font perdre taut de temps, et surtout remplacer pour les amateurs peu fortunés les traités si dispendieux d'Histoire naturelle, telle a été notre pensée en écrivant.

Cependant les entomologistes exercés qui voudront bien jeter un coup d'œil sur ces tableaux s'apercevrcnt facilement qu'ils ne méritent point le nom de compilation. - Outre que nos descriptions ont été (à bien peu d'exceptions près) faites sur la nature, et que par conséquent le fond nous en appartient, qu'en un mot nous n'avons fait que nous aider des auteurs qui nous ont précédés, sans pour cela les copier, nous avons encore ajouté nos propres observations à celles déjà faites avant nous. Voilà la partie complétement neuve de notre ouvrage, et celle dont nous sommes spécialement comptables à nos lecteurs. Passons donc rapidement en revue les changements que nous avons cru devoir faire tant aux méthodes de MM. Latreille, Ochsenheimer, Duponchel et Boisduval qu'à la
description et à la fixation des espèces.

Nous le répétons, rendre la science plus facile et moins coûteuse à acquérir, tel est notre unique but. Pour y parvenir nous avons dû chercher à en simplifier les éléments; c'est en élaguant des coupes générales tous les caractères surabondants ou peu visibles (1), et en réduisant le nombre des espèces que nous avons tâché d'y parvenir. Mais comme il est juste que le lecteur soit mis à portée d'apprécier tous les systèmes, nous avons conservé comme subdivisions et variétés les genres et espèces que nous avons retranchés; nous avons donc décrit avec soin les variétés souvent même peu tranchées et peu répandues, pourvu qu'elles aient reçu un nom de quelque auteur, mais nous avons donné plus d'extension à la description de ces variétés à mesure qu'elles deviennent plus intéressantes et qu'elles nous ont paru plus susceptibles de former un jour des espèces distinctes quand la découverte de la chenille sera venue lever toute difficulté. Au contraire nous avons restreint les articles qui concernent celles qui nous semblent presque accidentelles; il en est même que nous avons complétement omises, parce qu'elles ne présentent que de ces différences qui se rencontrent très-rarement, une seule fois peut-être, et que leur histoire ne servirait qu'à encombrer nos colonnes sans utilité. Mais il est une dernière classe de variétés que nous n'avons pas décrites; ce sont celles que tous nos soins et recherches n'ont pu parvenir à nous procurer, soit en nature, soit figurées, et qui soǹt cependant citées comme dignes d'attention dans les auteurs les plus respectables. Quoique leur nombre soit peu considérable, nous n'avons pas cru devoir priver les amateurs au moins de leur nom et de leur place, et nous avons laissé celle-ci en blanc, afin qu'elles soient décrites à leur rang par ceux de nos lecteurs qui seront assez heureux pour se les procurer.

C'est encore dans le but de rendre la classification plus intelligible que nous avons ajouté aux caractères principaux tirés de la forme des insectés sous leurs trois états (réunion commandée par l'état de la science) des caractères secondaires, plus variables sans doute, mais plus visibles pour des yeux peu exercés.

Cette distribution de caractères génériques dont plusieurs n'avaient pas été observés avant nous, la suppression de quelques genres

(1) Aujourd'hui qu'on multiplie presque ả l'infini les coupes génériques, cette simplicité paraîtra peut-être peu en rapport avecl'état de la science, mais on sait que, plus un genre est étendu, moins ses caractéres sont nombreux; on ne s'étonnera donc pas de la brièveté
de ceux-ci, qui ne peut, s'ils sont bons et suffisants, qu'être avantageuse.

Quant aux genres nouveaux qu'on fabrique tous les jours, ils ont certainement leur côté utile, en réunissant dans de petits groupes assez naturels les espèces analogues; mais, en diminuant la difficulté pour les caractères spécifiques, ils l'augmentent pour les caractères génériques, et nous croyons que l'élève ne saurait gagner au change. D'ailleurs cette multiplicité de genres n'a souvent d'autre mérite que de donner des noms à des divisions déjà fort bien établies par tous les auteurs, et d'autre cause que l'amour-propre des eréateurs; amourpropre qui, sagement ménagé, est le plus puissant auxiliaire de la science, mais qui, s’il n'est pas réprimé, en devient bientôt le plus redoutable ennemi. Sans prétendre ici blâmer personne, nous citerons un exemple entre vingt de l'inconvénient que présente, à notre avis du moins, cette marche trop suivie aujourd'hui.

Le genre Harpya d'Ochsenheimer, qui ne contenait que six espèces, fut partagé en deux par la création du genre Dicranura. Il ne restait plus dès lors dans les vérilables Harpya que trois espèces, Fagi, Ulmi et Milhauseri; la seconde vient d'être érigée en genre sous le nom d'Uropus. Nous n'avous donc plus d'Harpya que deux espèces, qui sont assez dissemblables sous leurs trois états pour nécessiter la formation d'un nouveau genre avec l'une d'elles; et, si l'on veut être rigoureusement conséquent dans cet esprit d'analyse, il y aura bien peu de genres. si peu nombreux qu'ils soient, qui ne semblent devoir éprouver le même démembrement. L'entomologie sera alors inabordable pour les commençants. 
trop peu caractérisés, et la création d'une nouvelle sous-tribu à laquelle il ne manquait pour ainsi dire qu'un nom, tant elle est naturelle (1); voilà, pour ce premier volume, tout ce qui nous appartient dans la méthode. C'est aux entomologistes instruits à juger si nous n'avons rien retranché d'utile, rien ajouté de superflu.

Quant aux espèces, tout le monde sent aujourd'hui le besoin d'arrêter ce débordement de créations dont on noie la science ; cependant, comme le nombre des découvertes réelles a prodigieusement augmenté depuis peu d'années, nous ne saurions en revenir à la simplicité de Linné et des premiers auteurs. D'un autre côté, cet accroissement augmente l'incertitude des caractères spécifiques, car on rencontre chaque jour des espèces qui font transition entre deux autres déjà connues, et qui, possédant plus ou moins de caractères de l'une ou de l'autre, rendent leurs descriptions fautives ou incomplètes. Il ne faut donc pas se dissimuler que la science, même abstraction faite de la cupidité des marchands et de l'amour-propre des amateurs, devient plus difficile de jour en jour, et que c'est plutôt par une espèce d'instinct qu'avec les descriptions ou les figures que les entomologistes parviennent à distinguer entre elles certaines espèces. Pour pouvoir se retrouver dans ce dédale, il faudrait, autant que possible, suivre des principes constants, tracer des limites bien déterminées pour l'établissement des espèces; voici notre système à cet égard.

Une espèce n'est suffisamment caractérisée que par des différences sensibles, constantes ou analogues dans des localités différentes, de l'insecte sous ses trois etats (ou du moins sous les deux extrêmes) d'avec les espèces voisines. Ce n'est que quand ces conditions sont réunies que tout doute est levé et qu'une espèce devient bien authentique (2). Il serait donc bien à désirer que l'état de la science permît d'appliquer rigoureusement ce principe. Dans les Nocturnes proprement dits, dont presque toutes les chenilles sont connues (nous ne parlons pas des Tinéites, Tortricines, etc.), nous espérons en faire une application exacte; mais dans une partie des Diurnes et des Crépusculaires (Lat.), elle devient tout-à-fait impossible : combien d'espèces en effet, surtout dans les genres Satyrus, Hesperia, Polyommatus, Sesia, dont on ignore et dont on ignorera encore bien long-temps les premiers états! Nous sentons qu'il nous est impossible d'ajourner la fixation d'une espèce à l'époque de la découverte de sa chenille, quoique, nous le répétons, elle n'ait pas acquis pour nous avant ce jour son caractère d'authenticité. Placés entre notre conviction à cet égard et la nécessité de décrire fidèlement des espèces très-distinctes dont les premiers états sont inconnus, nous tâcherons de suivre un parti tempéré, et nous prendrons en considération, pour fixer les espèces de Diurnes, leurs différences bien sensibles, l'invariabilité de ces différences, l'époque d'apparition, les mœurs, l'avis des auteurs les plus suivis, etc. Seulement, inclinant toujours vers le système que nous venons d'exposer, nous serons très-scrupuleux sur l'admission des espèces. Toutefois, comme nous voulons toujours mettre le lecteur en état de décider lui-même la question, nous ne supprimerons jamais une espèce déjà nommée, nous la joindrons seulement en la décrivant dans une accolade avec celle dont elle se rapprochera le plus, et si par la suite il devient certain qu'elle constitue une espèce pàrticulière, rien ne sera plus aisé que de l'isoler en considérant l'accolade comme nulle. En un mot nous laisserons au temps et aux observateurs le soin de décider la question dans la colonne que nous laissons en blanc à cet effet. De cette manière nous épargnerons à la mémoire des fatigues continuelles, et aux boîtes des collecteurs des vides considérables.

Quant au nombre des espèces que nous donnerons, il se présente une autre difficulté. Tous les iconographes qui ont décrit les papillons européens ont été fort embarrassés pour connaître la patrie de certaines espèces limitrophes. Il est assez facile, en effet, d'isoler les espèces des côtés du nord et de l'ouest où notre continent est bordé par des mers; mais, dans quelques parties du sud et dans l'est tout entier, la tâche devient très-épineuse. Comment acquérir la certitude que telle espèce qui vole au sommet de l'Oural ou aux environs de Constantinople n'y est pas arrivée accidentellement d'Orembourg ou de Scutari? Certains auteurs ont mis la plus grande réserve à admettre ces espèces voyageuses, d'autres au contraire semblent avoir pris à tâche de décrire non-seulement les papillons qui habitent nos pays, mais-encore ceux qui pourront y débarquer un jour. Sans prétendre blàmer aucun de ces deux partis, nous tâcherons de compléter nos tableaux sans toutefois y admettre des espèces trop disparates et d'un facies tout-à-fait exotique, surtout parmi les espèces nouvellement découvertes, les autres existant déjà dans la majeure partie des collections. On ne s'étonnera donc pas que, tout en décrivant les Col. Aurora, Satyr. Anthelea, etc, nous nous abstenions de donner non-seulement les Argyn. Niphe, Poly. Echion, Satyr. Clylus, qui sont maintenant bien reconnus exotiques, mais encore les Pap. Xuthus, Danaï Chrysippus, Alcippus, etc., qui n'ont aucun rapport avec les espèces du même genre ou avec les autres genres européens et dont la patrie est réellement étrangère, quoiqu'ils aient pu être trouvés accidentellement sur notre territoire. Au reste pour qu'on ne puisse nous reprocher d'être incomplets, nous décrirons à la fin du volume, dans un petit supplément et avec un numéro d'ordre, toutes ces espèces dont l'habitat est plus que douteux.

Voici maintenant quelques avis sur la manière de faire usage de nos tableaux :

(1) Voyez à ce sujet la note qui se trouve au bas de la tribu des Lycænides.

(2) On a quelquefois objecté à ce système de fixation des espèces par les chenilles, la différence des chenilles elles-mêmes dans une seule espèce. Mais, loin de détruire notre système, il nous semble que cet argument conclut au contraire en sa faveur. Ea effet, nous ne regardons pas comme des espèces de chenilles différentes les variétés constantes qu'on observe chez celles des Chel. Fuliginosa, Phlog. Alulatrix, Catoc. Nupta, Lasioc. Quercifolia, etc., par la raison qu'elles produisent indistinctement le même papillon. Pourquoi donc regarderions-nous comme espèces séparées les Cleopatra et Rhamni, Napi et Bryonice, Ilia et Clytie, Paphia et Valesina, puisqu'elles proviennent indistinctement de la même chenille? Ces raisonnements, sur l'un desquels tout le monde est d'accord, nous semblent se prouver l'un par l'autre. Personne au contraire n'aura l'idée de réunir deux espèces comme Triplasia et Urtica, Dictaea et Dictceoides, etc., quoique très-semblables, par la raison que la même chenille donne toujours le même papillon, et que le même papillon donne toujours naissance par la ponte à la même chenille. 
$1^{\circ}$. Pour pouvoir reconnaitre aisément un papillon sur nos descriptions, nous engageons les jeunes amateurs à avoir sous les yeux, non-seulement les caractères de l'espèce, mais encore ceux des différents groupes et divisions dont elle fait partie ; c'est à différencier entre elles les espèces les plus voisines. De même, les plus positifs, la description spécifique étant surtout destinée nos efforts tendent à exposer les différences de l'une à l'autre plutôt qu'à un groupe présente beaucoup d'espèces analogues, tous geons quelquefois les points de ressemblance les plus saillants. Ainsi, dans les Satyres blancs ète de l'individu, et nous néglipoint des yeux qu'on remarque au bord marginal des ailes inférieures en dessous, et dont les deux arge, Bdv.), nous ne parlons parce que ce caractère est commun à tout le groupe. De même dans les Polyommates Azurins (Cyx antérieurs sont isolés, etc. , que légèrement des points ocellés quand ils n'offrent pas de caractère spécifique, de cette section.

$2^{\circ}$. Nous donnons en tête de chaque description l'envergure de l'espèce expre en que cette envergure n'est que très-approximative. En effet, beaucoup plus petits, soit à cause du climat, soit à cause de non-seulement on rencontre dans une même espèce des individus même papillon étalé de deux manières peut offrir des envergures toutes différentes. Celles qudante des chenilles, mais encore le comme mesure exacte de la taille d'une espèce, que comme point des diférentes. Celles que nous donnons serviront done moins excellent ouvrage suit un autre système : c'est celui de comparaison avec d'autres. - M. Ochsenheimer dans son lui donne encore l'avantage de décrire d'un seul mot le port en tête d'une description une autre espèce de la même taille, ce qui souvent elles-mêmes inconnues aux amateurs inexpérimentés, nous espece; mais celles qu'il cite comme point de comparaison étant dant nous l'emploierons dans les Nocturnes, conperimentes, nous avons dû, à notre grand regret, renoncer à ce moyen. Cepen-

concurremment avec les envergures chiffrées.

ficultés typographiques, et par conséquent le prix de l'ouvrà la synonymie, mais nous aurions ainsi augmenté de beaucoup les difteurs les plus universellement suivis, ceux dont les ouvrages sont de fort peu son utilité. Nous avons donc seulement cité les auner, qui au second titre et quelquefois au troisième, a l'avantage sur tous les iconographes, ot qui donné les meilleures figures, Hubtieuses. Nous avons toujours eu soin de reste, aucun des auteurs que nous avons ces figures que nous avons auparavant scrupuleusement vérifiées, Au consulté la figure qui y correspond, que nous le menté légèrement, et ce n'est qu'après avoir lu attentivement sa description et $4^{\circ}$. Pour faciliter aux jeunes amateurs la recherche toujours dificile synonymique.

$4^{\circ}$. Pour faciliter aux jeunes amateurs la recherche toujours difficile des caractères généraux, nous avons disposé ces derniers
d'après une méthode analytique, comme on l'a fait dans ces derniers to d'après une méthode analytique, comme on l'a fait dans ces derniers temps pour les plantes, de telle sorte que, sans que l'ordre
des divisions soit dérangé, l'élève n'ait le choix qu'entre deux dagos aux plus petites sections sans risquer de se tromper.

$5^{\circ}$. Toutes nos descriptions sont faites sur des mâles, à moins que la femelle seule ne soit connue. A la fin de la description du mâle se trouve celle de la femelle, qui est comparative avec la première, c'est-à-dire que nous indiquons seulement en quoi elle differe du mâle, les autres caractères de la description lui étant communs.

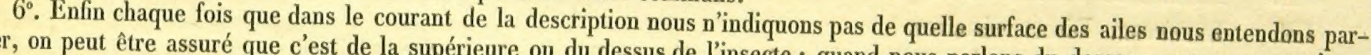

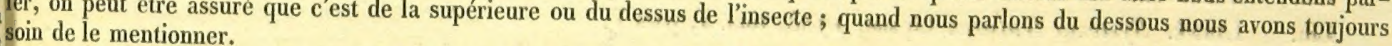

Pour rendre à chacun ce qui lui appartient, nous devons dire ici que nous avons puisé plusieurs bons renseignements dans les beaux ouvrages de MM. Treitschke, Godart, Duponchel et Boisduval. L'Iconographie des chenilles des deux derniers auteurs
uous a surtout fourni une foule de documents précieux.

Enfin nous ne terminerons pas sans adresser ici nos remercîments : A M. Alexandre Lefebvre, entomologiste bien connu, qui a eu l'extrême complaisance de nous confier, malgré l'éloignement
les dangers du transport, les espèces qui nous manquaient;

A notre compatriote, M. Marchand, qui a bien voulu nous laisser consulter sa riche bibliothèque et sa magnifique collection;
Et à M. Bugnion, de Lausanne, qui nous a fourni de nombreux renseignements sur les mœurs des espèces suisses, et en particu-
er sur celles des Satyres nègres. er sur celles des Satyres nègres.

\section{A. GUENÉE ET F. DE VILLIERS}




\section{LISTE}

\section{DES AUTEURS CITÉS DANS LE GOURANT DE CE VOLUME.}

Bdv. Borspuval. Europ. Lepid. Index methodicus. Paris, 1829.

- Icones des Lépidoptères d'Europe connus ou peu connus. Paris, 1832.

- Collection iconographique et historique des Chenilles d'Europe. Paris, 1832.

Bon. Bonelur. Descrizione de novi Insetti Lepidotteri, etc. Mémoires de l'Académie des Sciences de Turin.

Bork. Borkausen. Naturgeschichte der Europæischen Schmetterlinge, etc. Francfurt d̀ Mein, 1789 .

Curt. Curtis. British Entomology. London, 1824 .

Dalm. Dasman. Forsok till systematisk Uppstallning af sveriges Fjarillar (Kongl. veteuskaps academieus Handling for ar 1816 ).

Dup. Duponches. Supplément à l'Histoire naturelle des Lépidoptères de France. Paris, 1832. - Complément à l'Histoire naturelle des Lépidoptères de France (Chenilles).

Engr. Engrasrelle. Papillons d'Europe, peints par Ernst et décrits par Engramelle. Paris, in-4º

Esp. EsPer. Die Schmetterlinge in Abbildungen nach der natur. Erlangen, 1777.

Fab. Fabricius. Plusieurs ouvrages.

Fisch. Fischer. Entomographie de la Russie. Moscow, 1821.

Geoff. Grofrroy. Histoire abrégée des Insectes des environs de Paris. Paris, 1762.

God. Godart. Histoire naturelle des Lépidoptères ou Papillons de France. Paris, 1 S20. -Encyclopédie méthodique, Insectes, tom. IX. Paris, 1819.

Hub. Hubner. Sammlung Europaischer Schmetterlinge. Augsburg, 1805.

-Geschichte Europaischer Schmetterlinge gesammelt von J. Hübner (Chenilles).

Lat. Latreille. Plusieurs ouvrages.

Lef. Lefrebve. Divers mémoires.

Lin. Linné. Systema Naturæ. Lipsiæ, 1788, tom. I, pars V.

Ochs. Ochsenheimer. Die Schmetterlinge von Europa, tom. I, II, 1807-1808, et tom. IV, 1816 .

Treit. Treisschke. Continuateur de l'ouvrage précédent. 


\section{TABLEAUX SYNOPTIQUES}

\section{DES LÉPIDOPTĖRES D'EUROPE.}

L'ordre des Lépidoptères se divise en

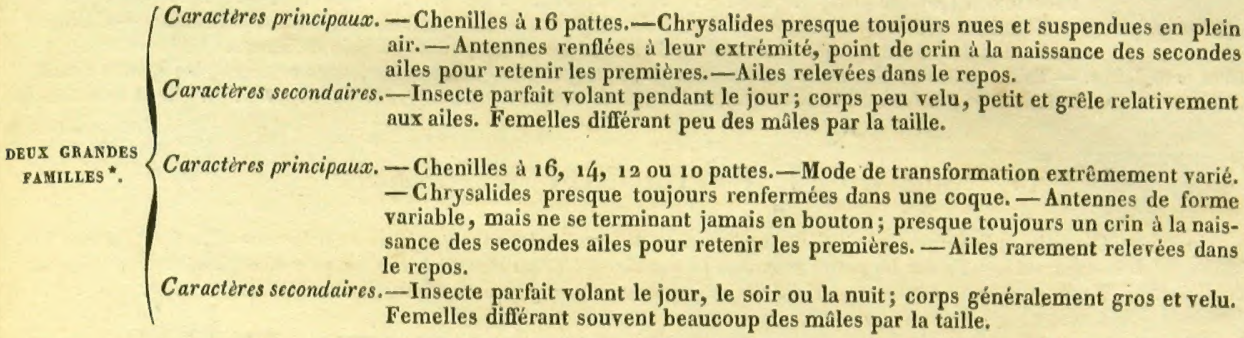

DIURNI.

Famille 1. DIURII (DIURNES).

(Lat. - Papilio. Lin. - Rhopalocères. Duméril. Bdv.)

Caractères principaux. - Chenilles à 16 pattes. - Chrysalides presque toujours nues et suspendues en plein air. - Antennes renflées a leur

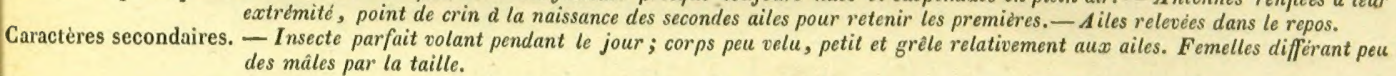
des mâles par la taille.

DEUX GRANDES (Jambes postérieures n'ayant qu'une seule paire d'épines; les quatre ailes élevées parallèlement dans le repos.

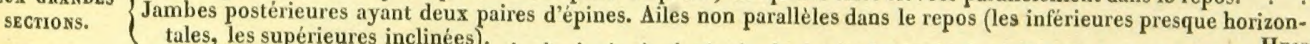
tales, les supérieures inclinées). . . . . . . . . . . . . . . . . . Hesperidi

(1) Jambes postérieures n'ayant qu'une seule paire d'épines, les qualre ailes élexées parallètement dans le repos. (2) $\left\{\begin{array}{c}\text { Chrysalides attachées par la queue et par un lien transversal au milieu du corps, presque toujours la } \\ \text { têt. }\end{array}\right.$

Chrysalides attachées par la queve seulement, la tete

NyMPHALIDI. $(72)$

- Nous partageons l'avis de M. Boisduval, qui pense que les Crépusculaires de Latreille doivent être réunis aux Nocturnes, et que leur séparation n’est pas fondée sur des caractères suffisants. Nous avions d'abord eu l'intention de ne donner sous le premier nom que les espèces dont les antennes ne décroissent point de la base au sommet, et par conséquent d'en retrancher les Procris pour les grouper avec les Emydia; mais un peu d'attention nous a démontré l'impossibilité de cette marche. Bien plus, nous avons su que le genre Procris lui-meme appartient, sous le rapport des antennes, aux deux familles; ainsi la $P$, Statices a les antenpes renflées au sommet, tandis que les mâles des P. Globularia, Pruni, etc., les ont au contraire presque semblables à celles des Emydia; d'ailleurs comment isoler les Procris des $Z_{y}$ gena, avec lesquelles elles se lient si bien par les $S$ yntomis et les $P$ sichotoe? Nous adoptons donc la division des Lépidopteres en deux famille Toutefois nous ferons observer que cette division est encore trés-artificielle, puisque les Diurnes se lient insensiblement aux Nocturnes par les Hesperia, Castnia. Coronis, Agarista, Egocera et Hecathesia. La famille des Diurnes ou Rhopalocères est donc loin de reposer sur des bases stables, et ne peut se caracteriser comparatiyement a l'immense famille des Nocturnes que par des diagnoses exceptionnelles et dont elle partage toujours l'une ou l'autre avec quelque tribu de ces derniers (Les antennes en massue avec les Zygana, l'absence du crin des deuxiémes ailes avec les Hépiales et certains Bombycites, les ailes élevées dans le repos avec plugieurs Phalénites, les métamorphoses et la forme des chenilles avec une foule de Gometra, Noctua, etc., etc.); c'est ce qu'exprime d'aillenrs évidemment le no

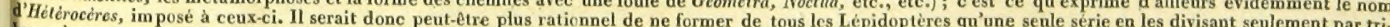
bus; c'est ce que, a près des études plus approfondies sur les exotiques, nous ferons peut-être dans la seconde édition, si le public veut bien épuiser celle-ci. Quant aux dénominations de Diurnes, Crépusculaires et Nocturnes, sans doute elles sont et ne peuvent etre qu'inexactes dans leur signification rigoureuse; mais Rhom est de si peu d'importance quand il s'agit de détruire des travaux si anciens, que nous croyons devoir conserver ceux de Diurnes et Nocturnes, le nom de Rihopalocères d'ailleurs (qui convient également à beaucoup d'Hétérocércs), s'il est moins impropre, n'étant pas plus exclusif,

I. 


\title{
(3) Tribu I. PAPILIONIDI (papillonides).
}

\author{
(Lat. God. - Succincti, Bdv.)
}

Chrysalides attachées par la queue et par un lien transversal au milieu du corps, presque toujours la tête en haut. .

Caractères principaux. - Chenilles cylindriques ou de forme ordinaire. - Chrysalides presque toujours anguleuses (a un

(4) \{ Caracteres secondaires. - Taille grande ou moyenne. - Fond des ailes ordinairement jaune ou blanc, jamais bleu *. genre près). - Crochets des tarses très-apparents. - Toutes les pattes propres à la marche. - Cellule discoidale des secondes ailes fermée.

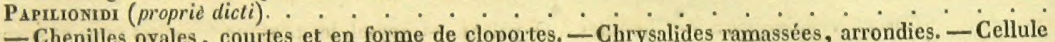
Caractères principaux. - Chenilles ovales, courtes et en forme de cloportes. - Chrysalides ramassées, arron

Caracteres secondaires. - Taille petite ou du moins au-dessous de la moyenne. - Ailes supérieures entières; les quatre ayant le fond bleu, faure ou brun, toujours très-rif en couleur.

\section{(5) Sous-tribu I. PAPILIONIDI (propriè dicti).}

Caractères principaux. - Chenilles cylindriques ou de forme ordinaire. - Chrysalides presque toujours anguleuses. - Crochets des tarses très-apparents. - Toutes les pattes propres d la marche.-Cellule discoüdale des secondes ailes fermée.

Caractères secondaires. - Taille grande ou moyenne. - Fond des ailes ordinairement jaune ou blanc, jamais bleu. .

( Chenilles portant sur le cou un tentacule en forme $d^{\prime} Y^{* *}$. - Bord abdominal des ailes inférieures échancré et formant à

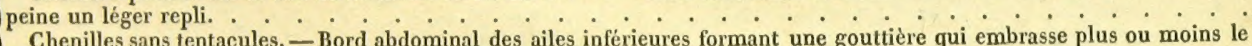
dessous de l'abdomen. . . . . . . . . . . . . . . . . . . . . . . . . . . ( . . . .

(7) Chenilles portant sur le cou un tentacule en forme d’Y.-Bord abdominal des ailes inférieures échancré et formant d peine un léger repli.

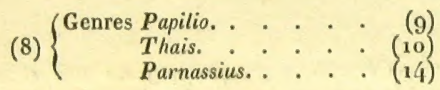

(9) Genre I. PAPILIO (papillon).

(Lat. Ochs. Bdv.-Amaryssus. Dalman.)

Caractères principaux. - Chenille rase. - Chrysalide anguleuse dète bifurquée. - Massue des antennes allongée. - Palpes trís-courts, dernier article point ou peu distinct.-Tete et yeux gros et saillants.

Caractères secondaires. - Taille grande.-Ailes a fond jaune avec des bandes noires, les supérieures entières, les inférieures largement dente avec deux queues très-longues et une tache ocellée d̀ l'angle anal.

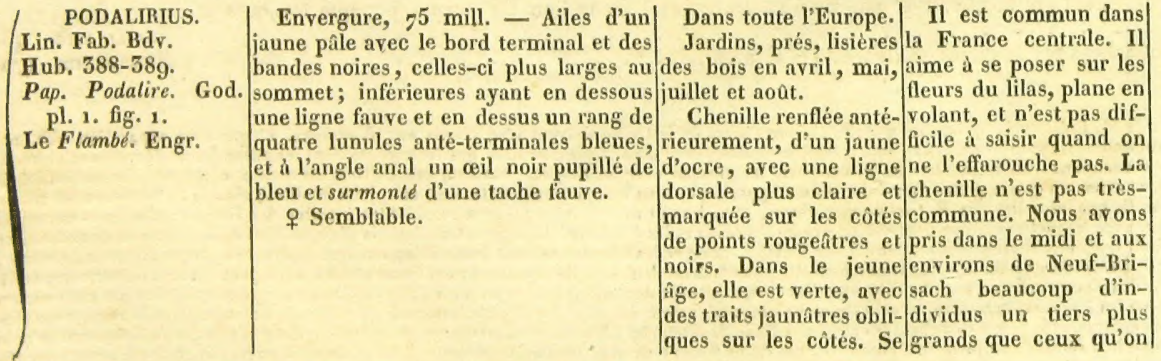

- Ces caractères secondaires ne sont applicables qu'aux espèces européennes.

* Pour voir ce tentacule il faut inquieter la chenille, car dans l’état de repos il est caché sous le premier anneau, d'où elle le fait sortir à volonté. 
Feistianecu.

Dup. Suppl. pl. Ordinairement un peu plus grand, fig. 1. trouve en juin et sep-|trouve aux environs de tembre sur te prunellier, Paris.

le pêcher, l'amandier.

Chrysalide d'un jau-

ned'ocre, avec des rangs

longitudinaux de points

grisâtres, la partie anté-

ricure plus foncée et le

dos foriement relevé en

pointe. Accrocliée aux

branches et aux murs.
Nous n'osons faire

La chenille, dont une espéce séparce de
I. Duponchel a bien ce Papillon, par la rai-

var. God. bord marginal d'un jaune bien plus foncé; voulu nous communi- son que la description Encycl. les inférieures ont le bord plus large- quer la description, est de sa chenille se rapment noir, les queues un peu plus lon-d'un beau vert, avec porte assez bien aux gues et la tache anale plus régulière, lles points ferrugincux, jeunes individus du $P_{0}$. plus vive en couleur et plus ocellée.
une ligne dorsale jaune dalirius. Cependant, il ct d'autres lignes obli- serait possible qu'elle ques latérales de la mê- en fat réellement difféme couleur. observations plus suivies nous apprendront. Le papillon vole, dit-on, sans se confondre arec Podalirius.

ALEXANOR.

Envergure, -5 mill. - Niles d'un

Dalmatie, Morée, ProIl aime les gorges Esp. Bdv. Hub. $787-$ $-88$. God. pl. A r. fig. 1 . tre bandes sur les supéricures, dont les gne.
te prend, posé sur des

des montagnes, où on deux discoïdales très courtes, et une sur Dans les montagnes, chardons dans la plus les inférieures, noirs; bord abdominal en juin et juillet. grande chaleur. Il ne longé par une autre bande de la même Chenille d'unvert pâ- paraît qu'une fois par couleur; bord cxterne marqué de taches le, arec les incisions an. Les individus de anté-terminales jaunes, surmontées de noires et ayant sur cha- Dalmatie et de Morée quelques lunules bleues; angle anal que anneau, excepté le sont plus grands que ayant un ocil noir, pupillé de bleu au-premier, des taches de ceux sle nos pays. dessous duquel est une tache fauve.

ㅇ Scmblable. même couleurentrecou-
pées de jaune. Se trouve en juillet, sur le Seseli dioicus.

Chrysalide grisâtre.

S'accroche à même les rochers.

MACIIAON.

Lin. Fab. IIub. 590-

Envergure, 80 mill. - Ailes jau-

Dans toute l'Europe.

Cette espèce, qui est Prés, bois, jardins, commune, a le vol raarec trois bandes courtes et noires à la champs. En mai, juil- pide, et est assez farou-
cûte, et un rang de taches lunulées de let, aoot et septembre. che quand elle a été 50 .

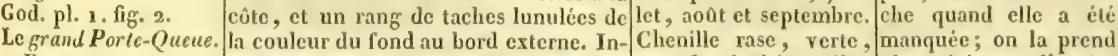
Engr.
férieures ayant au même bord un rang arec les incisions d'un plus aisément l'aprèsde taches bleues, et à l'angle anal un noir relouté, et sur cha- midi. Sa chenille est œil noir renfermant une tache fauve et que anneau, une bande commune.

un arc bleu.

o Semblable.

transverse de même Il est impossible de couleur, marquée de confondre ces trois espoints orangés. On la pices quand on se raptrouve en mai et sep-pelle que la tache fautembre sur le fenouil, la ve anale est au-descarotte saurage et celle sous de l'ocil dans $l$ ' $A$ des jardins.

lexanor, au-dessus dans

Chrysalide verte ou le Podatirius, et renferbrunâtre, avec deux mée par lai dans le Ma-

bandes jaunâtres ou chaon.
noirûtres sur le dos. 
$\mid$\begin{tabular}{c|c} 
Sprurgus. & $\begin{array}{c}\text { Ordinairement un peu plus petit et } \\
\text { ayant plus de taches ferrugineuses sous } \\
\text { les ailes inférieures. }\end{array}$ \\
Var. A. God. Encycl. $275-776$. & $\begin{array}{l}\text { Ailes d'un jaune très-foncé ; taille } \\
\text { plus grande, et oeil anal un peu obli- } \\
\text { téré. }\end{array}$
\end{tabular}

Nous arons vu cette prétenduc espéce enroyée d'Allemagne, et nous l'avons prise nousmême aux environs de Paris. Nous ne saurions la considérer même comme une variété. Germar figure (fasc. IV, tab. 15) une variété accidentelle fort remarquable.

Nous arons pris plusieurs fois celte variété aux environs de Valenciennes.

(10) Genre II. THAIS (Tinais).

(Fab. Latr. God. - Zerynthia. Ochs.)

Caractères principaux. - Chenille chargée d'ipines, charnue et velue. - Chrysalide efilde, terminée antéricurement par unc seule pointe. - Massue des antennes en cône ovale, allonge et un peu courbe, -Palpes de trois articles distincts dépassant le chaperon.

Caractires secondaires. - Taille moyenne. - Les quatre ailes dentelics, d fond jaune avec de nombreuses taches noires ct rouges, les inférieures ayant quelquefois plusieurs pelites queues. - Dessous presquo luisant. . . . . .

(11) Ailes inférieures ayant plusieurs appendices en forme de qucue. . . . . . (12)

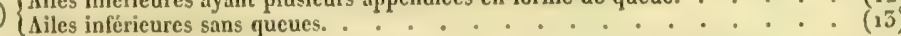

(12) Ailes inferieures ayant plusieurs appendices en forme de queue.

GERisyr. I' Envergure, 58 mill. - Ailes d'un| Grèce. En janvier, féGod. Hubn. Bdv. Icon. jaune pále, supérieures avec de nom- vrier et juin. pl. 2. fig. 1-3.

Dup. Suppl. pl. 2. fig: $1-2$.

breuses taches noires dont la plupart

partent de la côte et s'arrêtent à la nervure médiane. Inférieures a peine tachees de noir et ayant un rang anté-marginal de taches rouges dont la costale isolée et sous lesquelles on apercoit une ligne anté-terminale noire interrompue.

o Plus tachée de noir et ayant la ligne anté-terminale des inférieures continue, plus épaisse et marquée de petits atômes bleus au-dessous de chaque tache rouge.

HYPSIPYLE. Fab.? Bdv. Polyxena. Ochs. Hub $392-39^{3}$.

\section{(15) Ailes inférieures sans queues.} jaune serin clair avec une ligne termi- triche, Morie, etc.
Elle est encore rare dans les collections. Son apparition en juin (époque à laquelle elle a été prise par M. A. Lefebvre) semblerait indiquer qu'clle paraît dcux fois, tandis que ses congénères n'ont qu'une génération par an. Elle est très-commune aux environs de Smyrne. On la distingue toujours facilement des autres Thais par les queues de ses ailes inférieures.

Envergure, 58 mill. - Ailes d'un Italie, Espagne, $\Lambda$ u- Celte espèce ct la suivante ont long-temps nale trés-profondément festonnée, la base, Chenille d'un jaune été considérćes comles nervures et des taches noires. Supé- citron, arec une bande me identiques. N'ayant ricures ayant, avant la ligne festonnée, dorsale brune les épi- point élevé nous-mêune bande noire atteignant les deux nes fauves, cilices de me leurs chenilles, bords et précédée elle-même d'une au-noir, et une rangée la- nous ne pouvons assutre bande courte, partant de la côte où térale de points noirs rer qu'elles soient consclle est souvent marquée d'un point disposés triangulaire-tamment différentes, rouge; cellule contenant quatre grosses ment. Vit solitaire sur nous devons même dire taches dont celle de l'extrémité n'attei-l'aristoloche d̀ feuillesqque l'extrême ressemgnant jamais la côte et ordinairement rondes. On la trouve blance des insectes partraversée par une petite ligne jaune; en aont.

bord interne offrant deux bandes dont Chrysalide

faits nous laisse beauanguleuse coup de doutes at cet 
l'extérieure étroite, en zigzağ, et ne se [d'un brun jaunâtre pâle. jégard, néanmoins nous| liant pas ordinairement a la quatrieme tache de la cellule. Inférieures ayant, au-dessus de la ligne festonnée, un espace noir marqué de einq points bleus surmontés d'autant de taches rouges, et à la côte un trait rouge placé entre deux autres noirs et plusicurs taches noires assez petites sur le disque. Abdomen marqué de quatre lignes rouges longitudinales.

$\Varangle$ Plus grande et un peu plus marquée de noir.

\section{CASSANDRA.}

Hub. 910-913.

Bdy. Icon. pl. 3. fig. $1-2$.

Hypsipyle? God. pl 3. c. fir. 1-2.
La Diane? Engr. 109. A. B.

Dahl.

Envergure, 52 mill. - Ailes d'un Midi de la France, jaunc pate mais moins pur que celui de Environs d'Iyères, ctc. l'Ilypsipyle, avec les mêmes lignes et les En mars et avril. mêmes taches, mais ou le noir domine Chenille différant de davantage; deuxième tache de la cel- la précédente en ce que lule des supérieures très-étranglée au le fond est brunâtre, milieu, et tache du bout de la cellule avec les jointures plus atteignant ordinairement la côte; deuxiè-claires et que la série me bande du bord interne des mêmes latérale de points est ailes large, un peu lunulé et se liant su-remplacée par une ligne péricurement d lu tache du bout de la cel-plus foncée. Vit sur la lule; taches du disque des inférieures même plante.

plus marquées; points bleus plus petits. Chrysalide plus somEspace rouge du bord interne appuyé bre. inféricurement sur un trait blanc.

q Plus grande.

arons da nousen rapporter aux figures d'Ilubner et a l'opinion de II. Boisduval jusqqu'd $\mathrm{cc}$ que des expériences positives soient venues démentir leurs observations.

Voyez la note d'H $H p$ sipyle.

\section{(MEDESICASTE.}

Ochs. God. pl. $\tilde{5}$.

fit. 3-4.
llub. $63_{2}$. et Rumina. $594-395$.

La Proserpine. Engr. 109. A, B. C. D bis. Rumina. var. Bdy.
Elle ne differe sensiblement de Cassandra qu'en ce qu'elle est encore plus. marquée de noir.

Italie.

Nous arons ru cette Thails dans la collection de M. Marchand, qui l'a reçu de Dahl. Elle est à peine variété de Cas. sandra. Nous possédons une rariété analogue, mais plus remarquable en ce que les points rouges ont complétement disparu.

Enxergure, 45 mill. - Ailes un peu Lozere, etc., dans les quemment prise frele bord marginal noir divisé par des lu- garigues. En mai et telnau près M̂ontpellier. nules jaunes et de nombreuses taches juin. noires et rouges sur les quatre ailes. Elle plane a six pouces séric de deux ou trois taches apicales joun vert de terre, et est difficile blanches dur jaunâtre, arec deux ban- à apercevoir à cause de avec la bordure noireses. Inféricures des dorsales d'unjaune- sa couleur, qui la fait avec la bordure noire terminale plus soufre bordées de deux confondre arec lesplanespacée et formant deux lignes noi- lignes noires interrom- tes sur lesquelles elle res parallèles surmontées d'un rang de pues, et deux points la- se pose. Elle est comtaclies rouges. Dessous des inférieures téraux de même couleur mune dans les collecd'un blanc un peu nacré aree les dessins des deux côtés de cha-tions. du dessus. que anneat. Epines Sa chenille vient f Plus grande, avec les taches rou- orangées à sommité plus d'être découverte par ges ordinairement plus délayées. claire et cilices de noir. M. Duponchel.

Tête brune, arec deux taches plus foncées. Pattes écailleuses brunes. Se trouve i la fin de juillet, sur $l^{\prime}$ Aristolochia pistolochia.

Chrysalide semblable at celle de l'Hypsipyle. 
\begin{tabular}{c|c} 
Rowrsa. & Differo de Mrdesicaste par sa taille, \\
Lin Ochs. God. Bdr. ordinairement beaucoup plus grande, sa
\end{tabular} Hub. 633-65.

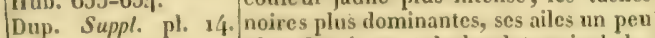
fir. 1-2. plus dentées, et le bord terminal des inféricures dont la ligne noire supérieure est beaucoup plus large, au point qu'elle s'avance jusque sur le disqque, et par cela même renforme les taches rouges au lieu d'en être surmontée; sa partic inférieure est aussi semée d'atômes bleuîtres.

\& Scmblable.

Hosmonati.

Differe de Medesicaste en ce que la Bdv. Icon. pl. 3. fig. plupart des taches noires ont disparu sur 3-5.

les supérieures, ou elles sont remplacées,

$T h$. Honnorat. Dup. savoir : la deuxième, à partir de la base, Suppl. pl. 2. fig. 3. par un point noir, et presque toutes les autres par de larges taches d'un rose vif bordées de' noir. Les inlérieures ont le disque entier du même rose, d̀ l'exception d'une tache basilaire, puis d'une médianc jaune.
Espagne, Portugal.

Environs de Digne.

Elle est très-rare, et ne nous semble qu'une rariété accidentelle de Medesicaste, quoiqu'on en ait trouvé plusieurs individus semblables.

\title{
(14) Genre III. Parnassius (parnassien).
}

\author{
(Doritis. Ochs. - Thais et Parnassius. Lat. God. - Doritis et Parnassius. Bdv.)
}

Caractères principaux. - Chenille pubescente. - Chrysalide arrondie, renfernée dans un lézer réseau. - Antennes courtes, d massue rrosse et oroide. - Palpes tres-zelus.

Caractères secondaires. - Taille grande ou au-dessus de la moyenne.-Les quatre aites entières, arrondies; les supericures presque depourcues d'écailles au bord marginal, dessous luisant. - Abdomen très-volu dans les mâles.

(15) Point de poche cornée sous l'abdomen des femelles, Dorrtis, Bdv.

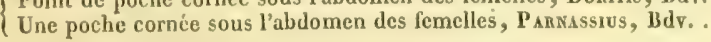

(16) Point de poche cornée sous l'abdomen des femelles.

Genre DonITIS. Bdv.

APOLLINUS.

Ochs. $13 \mathrm{dv}$.

Thais Apollina. God.

Dup. Suppl. pl. x. Gig. 2

Thia. Ilub. 635-656.

$68=-688$.

Le petit Apollon. Engr.
Envergure, 6o mill. - Ailes su-| Griec, Moréc, Calapéricures presque transparentes, sau-bre, sur les montagnes. poudrées de jaune et de noir et ayant $\Lambda u$ commencement du aussi en abondance aux a la côte deux grosses taches de celte printemps.

dernière coulcur; partie marginale transparente bordée d'une ligne jaune ombrée de noir, et peu sensible. Inférieures jaunes, avec le bord interne noirâtre, et sept taches anté-terminales blenes cerclees de noil et surmontées de rouge.

of $\Lambda$ ailes plus arrondics, les supérieures plus strićes de noir, les inférieures sablées de rouge et ayant les taches anté-terminales pliss marquées.
Elle est rare dans les aussi cn abondance at
cnvirons de Smyrne.

\section{(17) Une poche cornce sous l'abdomen des femelles.}

Genre PARNASSIUS. Bdv. God.

APOLlo. Envergure, 80 mill. - Ailes blan-| Alpes, Pyrénées, Lo- Quoique - ses ailes|

'́lin. Fab. Bdv. Inb. ches, les supéricures arec quatre ou zère, Suède, Laponic, soient très-entières, el$5,6-597,750-751$, cinq grosses taches noires, dont une cic. Sur les montagnes. les sont quelquefois telvers le milieu du bord interne aussilEn juin et juillet. |lement plissées qu'elles| 


\section{G. PARNASSIUS.}

God. pl. 2 B. fig. 1. $L$ 'Apollon. Engr. |prononcic que les autres, et une bande| Chenille d'un noir ve- paraissent un peu denanté-terminale d'atùmesnoirs atteignant louté seméde points d'un tées à cause des nervupresque le bord interne. Inférieures blanc bleuatre, avecune res qui rentrent en deavec une bande semblable, mais moins série latérale de taches dans. Il est trés-comprononcée, la base noire jusqu'auxdevs orangées. Tentacule de mun sur les montagnes tiers du bord interne, et trois à quatre la même couleur. Tête et descend même queltaches, dont deux grandes, rondes, d'un et pattes noirâtres. Se quefois en plaine, mais rouge vif, cerclées de noir et pupillées de trouve en mai, sur diflé- il n'est pas trés-facile blanc; les autres petites, réunies ou iso- rentes espéces de $S c d u m$ à saisir, quoique son lées à l'angle anal. Antennes très-cour- et de Saxifraga. Elle est rol soit fort lourd, partes, légèrement annelées de noir et de difficile ì élever. ce qu'il se tient ordinaigris.

Chrysalide arrondie, rement sur des endroits

o Plus grande, arec les taches rou-brune, saupoudrée de escarpés. Il varie prodiges ordinairement un peu jaunatres. gris bleuâtre, et renfer-gicusement. mée dans un léger tissu entre des feuilles.

Nomior. Fisch. Bdr. 4. $f$ ig. 3.

Dup. Supp. pl. द1. tig. 1.

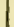

Tache costale externe et tache du pl. bord interne marquées derouge aux su- aoat. périeures. Base des inféricures marquéc d'une tache rouge; espace noir basilaire remontant fortement dans la cellule,

Russie orientale; en et y formant un crochet; frange mêlée de traits noirs longitudinaux. Antennes plus visiblement annelées.

q Plus sablée de noir que le ox.

PHOEBUS.

Falb. Bdr. God. pl.

Envergure, 62 mill. - o Ailes blan2 B. fig. 2.

Delius. Ochs.

Phabus. 567-568, et A pollo Delius. 6-19652. Hub. Var. Accid, 684-865.

HARDWICKIl.

IIope Zool. misc, tab. 4. fig. 1. et 1 a.

MNEMOSYNE

Lin. Fab. Bdr. IIub. 508.

God. pl. 2 B. fig. 3.

Le Semi-A pollon. Engr.

deux, et marquée supéricurement d'un

point rouge, et une bande courte anté-

marginale d'atômes noirs; point, ou bord interne. Inférieures sans bande anté-marginale, avec la base noire, joignant presque l'angle anal ct deux taches petites, rouges, ecrelées de noir, et très-rarement pupillées de blanc. $\Lambda \mathrm{n}$ tennes distinctement annelées.

o Plus noire, arec les taches plus grosses, dont une bien visible au bord interne, et souvent pupillée de rouge; sourent aussi une tache de celte conleur à la base des supérieures; la bande anté-marginale bien marquée, nême aux inféricures, qui ont aussi souvent le bord terminal peu fourni d'écailles. et enfin les deux taches anales comme dans $A$ pollo, mais presque toujours marquées de rouge.

La plus externe des taches costales est marquéc de trois points rouges ainsi que la tache du bord interne, et le bord marginal des inféricures est longé par une série de taches noires pupillies de blanc.

Envergure, 62 mill. - Ailes blan-

lule, puis un espace noirûtre; bord marginal largement dépourvu d'écailles at hes, les Alpes de la Suisse,

ches, les superieures avee trois taches de la seulement ruelques traces de taches an ches, avec les nervuresnoires, supéricu- se, Suéde, Sicile, etc. res arec deux taches noires dans la cel- En juin et juillet. sa partie supéricure, où il est ordinairement marqué de quelques taches blanches. Infericures avec la base et une
Cette variété intermédiaire entre Apollo et Phobus partage lous ses caracteres ayec l'une ou l'autre de ces espèces, qui varient tant. II est done absolument indispensable de connaitre la chenille pou en faire une espèce.

La taille est le principal caractere distinctif de cette espèce, qui varie autant qu'A pollo. Elle habite les prairies humides des montagnes, et se rencontre ordinairement le long des torrents qui descendent des glaciers. Elle est béaucoup moins répandue qu'Apollo, et ne se trouve que dans certaines localités: en Suisse, il faut la chercher dans le voisinage de $\mathrm{Bex}$, de Chamouny, du grand Saint-Bernard, elc. La rariété 684-685 d'Hubner est complétement dépourvue de taches rougesen dessus.

C'est une variété femelle dont la bande anté-marginale des inférieures est très-prononcée.

Il affec tionne les prairies humides des montagnes, et ne descend jamais en plaine. Pour l'avoir frais, il faut le chasser en juin. Les taches noires des inférieures (excepté celte do la 
G. PIERIS.

$\left|\begin{array}{l}\text { tache discoidale noires. Les quatre ailes } \\ \text { sans aucune tache rouge de part et d'au- } \\ \text { tre. Antennes presque entièrement noi- } \\ \text { res. } \\ \text { q Plus obscure, et ayant le plus sou- } \\ \text { rent une bande anté-marginale peu sen- } \\ \text { sible d'atômes noirs sur les inférieures. }\end{array}\right|$

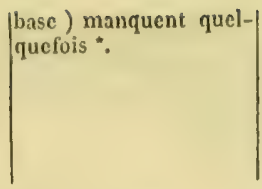

(18) Chenilles sans tentacules. - Bord abdominal des ailes inférieures formant une goutticre qui embrasse plus ou moins le dessous

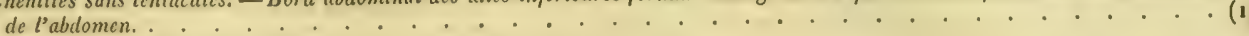

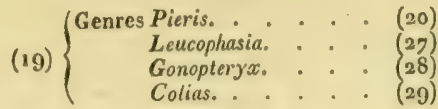

(20) Genre IY. PIERIS (PIÉRIDE).

(Lat., God. - Ponlia, Fab., Ochs. - Pieris et Anthocharis, Bdv.)

Caractères principaux. - Chcnille pubssconte ou mime un peu velue. - Chrysalide terminéc antéricuremont par une seule pointe, arec le dos renfle et le plus souvent carene. - Palpes cylindriques peu comprimés, assez longs, droits; massue des antennes ovoide; cellule discoidale de forme ordinaire. - Corps de grosseur moyenne. - Abdomen ne dípassant pas

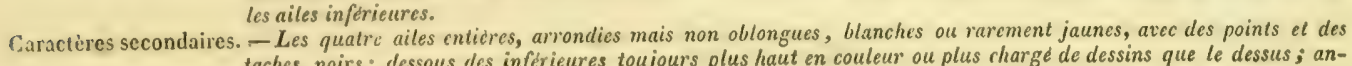
taches noirs; dessous des inferieures toujours plus haut en couleur ou plus rharge de dessins que le dessus; antennes souvent annelées de blanc et de noir. . . . . . . . . . . . . . . . . . . . .

(Chenille relue sur le dos, vivant sur les arbres. - Chrysalide un peu arrondic, terminée antéricurement par une pointe légèrement obtuse. - Liles légìrement allongées, sèches, peu chargées d'écailles en dessous, blanches avec des nervures noires. - Intennes d'une seule couleur, it massue presque insensible, peu renflée, fusiforme.. . . . . . . . . 2) Chenille pulescente, virant sur les plantes, principalement sur les crucifères. - Chrysalide anguleuse terminée antérieurement par une pointe aiguë. - Ailes peu chargées de dessins, les inférieures rarement veinées de noir en dessous, mais jamais marbrées de vert. - Antennes trés-annelées de blane et de noir, terminécs par un bouton aplati.
Chenille pubescente. - Chrysalide terminée par une pointe de forme variable. - Ailes inférieures marbrécs de vert en dessous. - Antennes légèrement annelées, terminées par un bouton aplati.

(22) Chenille velue sur le dos, vivant sur les arbres. - Chrysalide un peu arrondie, terminée antérieurement par une pointe legècment obluse. Ailes ligeircment allongées, sèches, pen chargícs d'cicailles en dessous, blarchics avec des nervures noires. - Antennes d'une seute couleur: a massue presque insensible, peu renflé, fusiforme.

CRATARI.

Lin. Fab. Ochs. Bdr. Hub. $599-400$. Envernue, 65 mill. - Ailes blanches, arec les nervures noires de part champs, prés, jardins. et d'autre, sans dessins ni points; extré- En juin et juillet.

Isa chenille et le pamité des nerrures noirâtre et peu four-

Pier. de l'aubipine. God pl. 2. fig. 3 . Le Gaze. Engr. nie d'écailles, surtout aux supérieures. o Semblable. muns La première lait e cylindrique, beancoup de tort uisante, couverte de arbres fruiticrs.

poils blanchatres, arec Cette espece a un fales côtés d'un rrisplom- cies tout diflérent des bé, et le dos noir, arec autres $\boldsymbol{P}$ iérides sous ses deux lignes longitudi- trois états, et mériterait nales faures. Vit en so-de former un genre d ciété sur l'aubépine plus juste tilre que beau(Cratcogus oxyacantha) coup de Piérides qu'on et les arbres fruitiers. a isolées du genre priOn la trouve en arril et mitif. mai.

Chrysalide d'unblanc verdatre, avec deux lignes latérales jaunes et

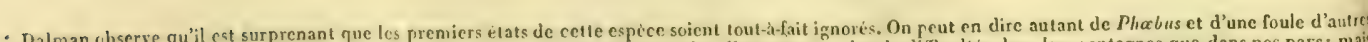

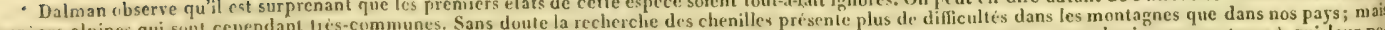

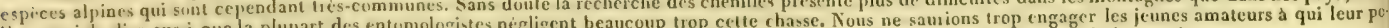
sitir n le permet, a s'y livier avec ardeur. Une ficule de diccuiertes et d'interessantes captures les dedummagera amplement de leurs pejaes.
} 


\section{G. PIERIS.}

beaucoup de taches noires, dont un rang sur l'envcloppe des ailes, qui est bordée d'une ligne dentelée de la même couleur.

(22 bis) Chenille pubscente, vivant sir les plantes, particuliement sur les crucifires. - Chrysalide angulouse, terminco antiricurement par une pointe aiguë. - Ailes peu chargies de dessins; les infírieures rarement veinées de noir en dessous, mais jamais marbries de rert. - Antennes tres-annelées de blanc et de noir, terminées par un bouton aplati.

BRASSIC 2 . Hub. 401-403.:Picr. du chou. God. pl.

$L e$ grand Papillon du leux gros points woirs; dessous des inchou. Engr.

Envergure, 65 mill. - Ailes blanLin. Fab. Ochs. Bdr. Dans toute l'Europe, On rencontre quelcal larcome jardins, prai-quetois des mâles qui cal largement noir. Les inféricures arec ries. Pendant toute li sont marqués en dessus ane tache de la même couleur au bord belle saison. de deux points noirs. inteme; dessous des supérieures avec Chenille d'un vert Hubnerdonne, comdeux gros points moirs; dessous des in- jaunatre, arec trois raies me ćtaut d'Lurope, la féricures jaune, sablé de noir. jaunes longitudinales, Pieris Cheiranthi (God. of Femelle ayant les deux points séparées par des points Bdr.), qui se trounoirs des supéricures apparents en des- noirs tuberculeux, de ve ordinairement it Tésus, une tache de cette couleur au bord chacun desquels s'élève nériffe; comme les auinterne, et les inféricures teintées de jau- un poil. Tête bleue pi- treshuteursn'en parlent ne aussi on dessus.

Lin. Fab. Ochs. Bdv. IIub. 404-405. Pièr. du chou. God. pl, 2. tert. fig. 2. Le petit pap. du chou. Engr. Hub. 904-907.

Lin. Fab, Ochs. Bdv. Hub. 406-407. pl. Gavet. God. Le papillon blanc veiné de noir. Engr. 104. a. b. c. d. quée de noir. Vit en so-pas, et que rien ne nous ciété sur le chow, et sur autorise à la considérer les autres plantes de la comme Européenne, famille des cruciferes. nous ne la comprenons Elle est très-commune, pas dans ces tableaux. ainsi que le papillon. (Yoyez le suplément, à Chrysalided'un blanc la fin de ce volume.)

sale, marquée irréguliè-

rement de noir et de jaunâtre.

Envergure, 45 mill. - Ailes blan- Dans toute l'Europe, Cette espèce est trèsches, les superieures ayant l'angle ipi-champs, jardins, prai-commune, et est toncal tigtremenì noir, le reste comme dans ries, etc. Pendant foute jours beaucoup plus pea précédente.

o Différant du ơ par les mêmes ca- $\begin{aligned} & \text { la belle saison. } \\ & \text { Chenille rerte, arec on rencontre plus sou- }\end{aligned}$

Chenille rerte, arec on rencontre plus soudente.

tudinales, dont les deux desmales tachés de noir latérales souvent inter-en dessus. Elle affecrompues. Tête verte. tionne, ainsi que la prèVitsolitaire,sur la grosse cédente, les lieux et rave, la capucine, etc. les temps un peu huChrysalide d'un gris mides.

sale, pointillée de noir,

et ayant sourent des teintes rosées.

Beaucoup plus petite, ailes plus ar- Dalmatie. rondies; tache inférieure du disque des premières ailes et tache de la côte des secondes manquant dans les deux sexes; dessous entièrcment dépourvu de taches discoildales noires dans les deux sexes.

Enyergure, 40 mill.- Ailes blan- Dans toute l'Europe, ches, les supérieures arec l'angle api-bois, prairies, jont de cssous des inférieures d'un jaune pîle, et l'été. avec des larges veines d'un noir verdatre Chenille d'un vert|sous des ailes inférieusuivant les nervures, et qu'on apercoit obscur, plus clair sur res bien plus coloré et confusément en dessus. lescôtés, avec les stig-plus veiné que dans ㅇ Différant du of comme dans les matesroux entourés d'u-toute autre localité. précédentes. $\quad$ ne petite tache jaune, Quelquefois la femelle des petites verrues blan-|a deux points noirs sur| 


\begin{tabular}{|c|c|c|c|}
\hline & 10 & $\begin{array}{l}\text { ches, des points noirs, } \\
\text { et un léger duret. Vit } \\
\text { solitaire sur le navet, } \\
\text { le réséda jaune, la tou- } \\
\text { rette glabre, la capuci- } \\
\text { ne, eto. } \\
\text { Chrysalide très-an- } \\
\text { guleuse, grisatre, et } \\
\text { pointillée de noir. }\end{array}$ & les ailes supérieurcs. \\
\hline $\begin{array}{l}\text { Napes. } \\
\text { Esper? Nobis. } \\
\text { Hub. 664-665? }\end{array}$ & $\begin{array}{l}\text { Cette variété est plus grande que } \\
\text { l'espèce ordinaire, et n'a que quelques } \\
\text { veines noirâtres et courtes sous les ailes } \\
\text { inférieures. Ses ailes sont aussi plus } \\
\text { épaisses et plus arrondies que dans l'es- } \\
\text { pèce typique, et la o a constamment } \\
\text { deux gros points noirs souvent accom- } \\
\text { pagnés d'une liture au bord interne. }\end{array}$ & $\begin{array}{l}\text { Environs de Paris. } \\
\text { Aoat, septembre. }\end{array}$ & $\begin{array}{l}\text { Nous avons souvent } \\
\text { pris cette rariété aux } \\
\text { environs de Chartres, } \\
\text { où elle ne se montre } \\
\text { que dans l'arrière-sai- } \\
\text { son, et toujours en } \\
\text { moins grande quantité } \\
\text { que l'espece principa- } \\
\text { le. Est-il bien certain } \\
\text { qu'elle provienne de } \\
\text { la même chenille que } \\
\text { Ia Napi? En tout cas, } \\
\text { elle fait le passage de la } \\
\text { Pieris Rapa d̀ l'espece } \\
\text { typique. }\end{array}$ \\
\hline $\begin{array}{l}\text { Bryonix. } \\
\text { God. pl. } 5 \text { E. fig. } 1 . \\
\text { Var. Napi. Bdv, God. } \\
\text { Encycl. } \\
\text { Hub. } 407 \text {. } \\
\text { Engr. 104. a bis. }\end{array}$ & $\begin{array}{l}\text { Variété q qui diffère de l'espèce en } \\
\text { ce qu'elle est d'un gris jaunâtre, sau- } \\
\text { poudrée d'atomes bruns, et en ce que } \\
\text { les nervures y sont très-dilatées en } \\
\text { dessus. }\end{array}$ & $\begin{array}{l}\text { Alpes, Yosges, Sty- } \\
\text { rie, Laponie, Suede, } \\
\text { sur les montagnes. }\end{array}$ & $\begin{array}{l}\text { Après avoir érigé en } \\
\text { espéce cette rariété, } \\
\text { Godart reconnut son er- } \\
\text { reur dansl'Encyclopédie, } \\
\text { et la rapporta da la Napi, } \\
\text { arec laquelle on la trou- } \\
\text { re souvent accouplée. }\end{array}$ \\
\hline
\end{tabular}

(25) Chenille pubescente. - Chrysalide termine par une pointe de forme variable. - Ailes inférieures marbrées de vert en dessous. - Antennes légèrement annelés, terminées par un bouton aplati. . . . . . . . . . . (.

(20) Point de tache aurore au sommet des supérieures dans les máles. . . . . . . . . . . . (25)

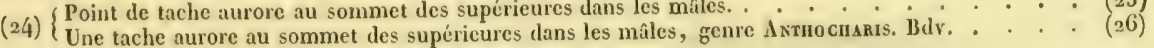

(25) Point de tache aurore au sommel des superieures dans les mâles.

CALIIDICE. Ochs. Bdv.

Hub. 408-409.

God. pl. E Y. fig. $2-5$.
Envergure, 42 mill. - Ailes blanches, avec une bandelette noire à la côtc des supéricures, et leur sommet marqué de deux rungs de points noirs; des-

Alpes, Pyrénées. En villet et aoot. Chenille d'un gris trouve que sur les monqué de deux rangs de points noirs, des-bleuâtretrés-foncé, poin- táones, à une grande avec treize taches sagittées, d'un jaune tre raies blanches, mar- a récemment découvert pâle.

I ailes plus arrondies, et ayant au chaque antieau d'une Ici devrait se plabord terminal, en dessus, une série de tache citron. Stigmates cer la Pieris Raphani taches ovales blanches, sur un fond d'un blanc brunftre. (God.Ochs. Dup.), mais noil.

Tête de la couleur dultous ces auteurs ont décorps, marquéc de cha- crit, et nous avons reçu que côté d'une tache nous-même souscenom jaune. Pattes écailleu-l'Hellica d'Hubner, qui ses noires. Vit en aoat n'habite pas l'Europe. et septembre, sur les Cependant, est-il bien crucifères, auprès des certain que la Raphani neiges permanentes. d'Esper, qui, d'après la Chrysalide grisâtre, figure qu'en donne cet finement pointillée de auteur, en differe notanoir, arec le dos mar-blement, puisqu'clle a qué d'une ligne jaune. quelques rapports avec la $L$. Sinapis, ne soit pas une espèce distincte? N'ayant pas vu cette 
Lin Fab.

IIub. 414-415.

God. pl. 2 secund. fig. 3. et 2 quart. fig. 2. I. Papillon blanc mar bre de vert. Engr.

Brahm.

BenLidice. $9^{5} 4$.

Cinlonidice.

Fisch. Bdy. I con. pl. 6. fig. 5-6.

Hub. 712-7 15 ?

Dup. Suppl. pl. 4. fig 3-5?

Daplidice, var. God Encycl.

Ochs. God.

Hub. 412-415.

Dup. Suppl. pl.111. fig. $1-2$.

Bdv. Icon. pl. 6. fig. $1=2$.
Envergure, 45 mill. - Ailes blan- Dans toute l'Europe, ches, angle apical des supérieures noi- dans les champs, incultes. En mai, ge, coupée par un trait blanc et saupou- juin et juillet. rin cendrélannées sans la revoir périeures jaunatre á la base, celui des bleuâtre, couverte de dans les lieux où on la inférieures d'un vert jaunâtre piqué de petits tubercules noirs prenait aupararant assez noir, avec des taches blanches dont les arec quatre raies blan-abondamment. marginales disposées en bande maculai- ches marquées à chare, celles qui les précédent en bande que jointure d'une tanon interrompue, et les autres, au nom-che citron. Ventre et bre de trois seulement, en triangle. pattes blanchatres, avec of Ayant les ailes supérieures plus une tache jaune au-desmarquées de noir, une tache de cette sus de chacune d'elles. couleur au bord interne tant en dessus Vit sur plusieurs réséqu'en dessous, et les dessins des infé- dacées et crucifères, en rieures répétés en noir en dessus.

juin et septembre.

Chrysalide grisâtre, pointillée de noir, avec quelques raies roussátres.

Un peu plus petite, angle apical Suisse, Russie, HonJes supéricures marqué de noir moins - étendu, moins intense et plus saupou- tres.

dré de blanc; dessous des inféricures d'un vert plus uni, moins mêlé de jaune, et ayant la bande blanche centrale noncontinue mais maculaire, surtout au bord interne; dessous des supérieures ayant la tache noire du bord interne fort peu marquée (dans le mâle) et sourent tout-à-fait oblitérée et n'ayant point de jaune a la base.

옹 Analogue à celle de Daplidice.

Elle est plus petite que Daplidice; Environs de Moscou. aigu même dans la femelle; la tache costale n'est point saupondrée de vert

- en dessous, et les taches noires du dessus sont plus petites et plus isolées; enfin les parties vertes du dessous des ailes inférieures sont d̀ peu près du même ton que dans Bellidice, mais clles sont plus étroites et plus allongées, ainsi que les taches blanches.

Envergure, fo mill. - Ailes blanches, avec le sommet des supérieures noir, traversé par une bande maculaire mars.

- blanche, tache costale noire, carrée et irayersée par une ligne blanchatre; dessous des supérieures ayant au sommet trois bandes légdrement nacries et bien tranchées sur un fond vert. Dessous des inférieures d'un rert foncé, arec des bandes légèrement nacries, transverses, inégales et netlement coupées.

$\uparrow$ Semblable. dernierc espèce en nature, nous ne pourons nous prononcer sur cettc question.

Celte espèce, sans très-commune; on est quelquefois plusicurs

( 
chs. God.

Hub. 546-547.

Dup. Suppl. pl. 5. fig. 5-4.

Bdv. Icon. pl. 6. fig. 3-4.
Envergure, 43 a 45 mill. - $\Delta \mathrm{i}-$ les blanches, arec l'angle apical des supéricures noir, traversé par une bande

blanche maculaire. Tache costale noirc un peu oblongue et traversée par une ligne blanchâtre. Dessous des supérieures ayant au sommet trois bandes inégales, peu tranchées, sur un fond rert. Dessous des inférieures d'un vert jaunâtre, piqué de noir, arec des bandes blanches, transrerses, très-inégales, et moins nettement coupées que dans Belemia.

o Semblable.
Espagne et Portugal. in fevrier et mars.

tevier et mars.

Cette espèce est \& la Belemia ce que l'A usonia est à la Belia. C'est dire qu'elle s'en distingue par son fond d'un vert plus jaunâtre et ses bandes moinstranchies. En la regardant d'un peu loin, on aperçoit sous les ailes inférieures une espece de ligne jaune qui part de la base et se prolonge jusqu'au bord externe. Elle n'est pas moins rare que la précédente. On la trouve aussi en Egypte.

Envergure, 40 mill. - Ailes blan- France méridionale,

Cette espèce, comFab. Bdv. Hub. 417 ches, les supérieures anguleuses, avec le dans les garigues, les et 418 (la fig. 416 est l'Ausonia).

God. pl. F 6. fig. $1-2$.

sommet noir et traverse par une bande jarding
maculaire blanche; tache costale assez avril.

large, marquée en dessous d'un S blane;

côte piquée de noir. Inférieures sans

taches en dessus, et ayant le dessous

d'un vert fonce, avec un grand nombre de

taches irrégulières d'un blanc nacré.

오 $\Delta$ yant le dessus des inférieures un peu jaunâtre.

Var. A. Nobis.

Un peu plus grande, tache costale petite et isolée; dessous des secondes ailes et sommet des supéricures d'un vert plus jaunâtre, arec les taches nacrées plus allongées, de sorte qu'elles forment des bandes assez régulières, quoique moins distinctes qne dans Belemia et Glauce.

TAGIS.
Bdv. Icon, pl. 5. fig. 1-3.

Bellesina. Bdv. Index. Dup. Suppl. pl. 3. fig.

5-6.
Belledice. Hub. 929930. Ramb. Hub, 560 ?

Envergure, 34 mill. - Ailes blan- Midi de la France,

Ticis. Midi de la France,
Provence. En avril et avec le sommet noir, marqué de trois mai. Chenille verte, avec chenille, due a le de sa le ventre plus pâle et zel. C'est la plus petite ment par une liture la côte qui est pi- une bande blanche laté-du grenre.

costale étroite et lunulée, joignant rare-

quée te noir. Dessous des inférieures rale surmontée d'une Nous croyons, ainsi d'un vert un peu jaunâtre, avec des ta-ligne d'un rouge vif. que nous l'arons dit it ches blanches non nacrées, et un petit Tête et pattes vertes. l'article Belledice, qu'il point noir discoïlal.

\& Semblable.

Vit solitaire, en juin, sur l'Iberis pinnata. 政, tirant postérieurement dice se rapporterait alors sur le rose, arec une li- fort bien ì la nôtre, a gne dorsale brune et la l'orthographe près, et sa pointe antérieure très- Belemida à notre T'agis, effilée.

Un peu plus grande, tache apicale Hub. 565-des ailes supéricures moins marquéc de
Corse, Portugal? a laquelle elle ressemble parfaitement.

C'est à peine une varićté de la nôtre; mais| 
Dup. Suppl. pl. 4. fig. blanc; taches blanches du dessous des I-2?

God.P Ochs.?

inféricures moins larges et quelquefois un peu nacrées.

\section{ACSONIA:}

Ochs Gorl. t. 2. pl, 6 E. fig 3-4.

Bdv.

Belia. Hub. 4,6

Envergure, 45 mill. - Ailes blanches, les supéricures avec le somme s. ches confuses; tache costale de grandeur moyenne, irrégulière et n'atteignant pas la cốte, qui n'est jamais piquée de noir en dessus. Dessous des inférieures d'un blanc ordinatirement sans éclat, avec des espaces leger's, irréguliers, et d peine contigus, d'un vert tressaupoudré de jaune.

o Semblable, et ayant seulement le dessus des inférieures teinté de jaunátre.

Simplonia. Bdv. Icon. pl. 5. fig. 4-6. Dup. Sup 3-4. . 5. fig 3-4.
Ausonia. Hub. 582 - glée et réniforme; base des inférieures
glétan583. et Marchanda. plus noire, dessous des mêmes ailes $9^{56}-9^{3} 7$.

.

plus marqué de vert et moins saupou-

Un peu plus grande, sommet des

costale, qui est marquée dans son milieu dré de jaunc.

q Ayant les taches costales et apicales encore plus noires et très-grosses en dessus. supérieures moins aigu, côte sablée de lais, Simpes du Va moir et presque atteinte par la tache En juin et juillet.

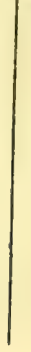

France centrale et
méridionale, dans les lieux secs. En juin. 
(rin EUPUENO

Lin. Fab. Bdv.

Hub. 421.423 .651 . God. pl. 5 e. $f_{g} \cdot 4-5$.

$L$ 'A urore de Provence. Engr.

Esp. Eupher God. Bdv.
Euvergure, 58 mill. - Ailes d'un , les méricures avec - un doc entiro point discoidal noir et une large tache pellier, Lozère, etc aurore cncadrée de noiratre. Dessous Dans les garigues, en des inférieures jaune mêlé de blanc, avxil et mai.

avec quelques bandes tranverses d'un vert noiratre. ayant seulement l'extrémité des supé- de points noirs. Vit sur rieures marquée de jaune orangí.

Le sommet des premières ailes est far. Yerdâtre en dessus, avec une tache aurore ovale et ublique, précédéc en dehors de quelques points blanes dont un isolé et plus gros. La raie verte en zigzag du dessous des inférieures s'approche davantage de la base et du bord postérieur. les cótés du corps blancs

la Biscutella Dydima.

minent et it anneaux de l'abdomen immobiles. Elle est blanchatre ou brunâtre, striée de lignes plus claires. pide, et aime à se reposer sur les cruciferes. Elle est commune, mais on trouve généralement plus de mâles que de femelles. Cette observation s'applique également à la Cardamines. Hubner donne (425) une rariété correspondante à celle de la précédente.

Nous n'arons point vu en nature cette variété, dont nous empruntons la description à l'Encyclopédie methodique. Voyez le Suppl it la fin du volume.

\title{
(27) Genre V. LEUCOPHASIA * (Levcoriasie).
}

\author{
(Bdv. Pieris. Lat. God.-Pontia. Ochs.)
}

Caractieres principaux - Chenille pubescente. - Chrysalide terminće par une seule pointe, avec le dos peu renfé et non caréné. - Palpes un pe recourbis. - Massue des antennes termince par un bouton ovale aplati. - Cellule discoidale, tres-courte et n'atteignan pas méme le tiers de l'aile. - Ailes très-oblongues. - Corps petit et peu velu. - Abdomen grêle, non velu, long et dépas sant les ailes inférieures.

Caractires secondaires. - Ailes minces, les infirieures non marbrées de vert. - Taille au-dessous de la mojenne.

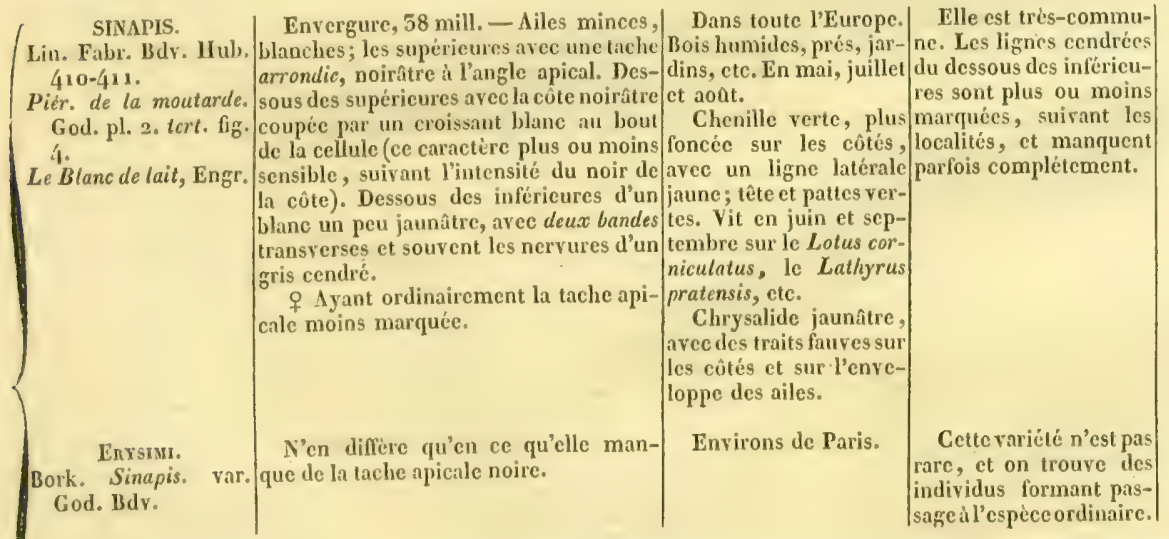

- Beaucoup d'autcurs, et en pasticuliex Dalman (Forsoh till Systematish, cle. Actes de Stochholm, ann. 1816, pag. 4S), se sont aperçus ele l'anomalie que pr

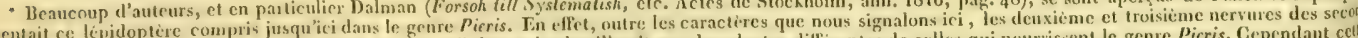

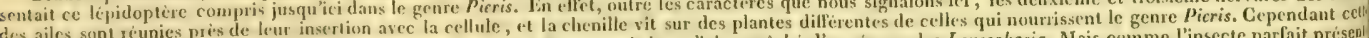

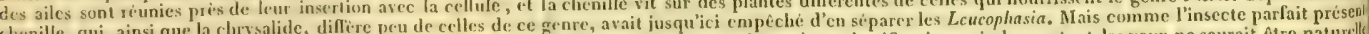

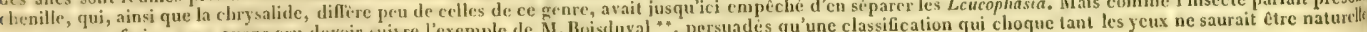
un lcut autre facies, nous avons cru devoir suive l'exemple de M. Boisduval "*, persuade 
Lathy:. Hub. $597-798$.

Pier. de li Gesse. Dup. Suppt. pl. 43. fig $4-5$.
Ailes supérieures coupées un peu/ Provence, Langueplus carrément. Tache apicale triangu-doc, Lozèrc, Suisse, etc. laire et descendant presque jusqu'au bord interne; base des quatre ailes d'un jaune soufre; dessous des inférieures complétement envahi par le gris cendré, à l'exception de deux taches blanches, l'une petite et incertaine, près de la base; l'autre grande, triangulaire, bien arrêtée inférieurement et joignan le bord terminal.
La tache de la base des secondes ailes en dessous est quelquefois presque effacée. La $\boldsymbol{L}$. Sinapis varie tellement que nous croyons indispensable de connaître la chenille de la Lathyri avant d'en faire une espèce. Nous avons pris abondamment prés de Neuf-Brisach une variété qui se rapproche celle-ci.

\section{(28) Genre VI. GONOPTERYX * (govoptenyx).}

(Leach. - Rhodocera. Bdr.-Colias. Lat. God, Ochs.)

Caractères principaux. - Chenille chagrine, comprimèe en arriere, à ventre plat. _Chrysalide à ventre extrimement saillant, et ¿i partie antérieure terminèe par une pointe très-aiguë un peu arquée. - Ailes très-anguleuses. - Palpes comprimés, peu velus, leur dernier arlicle peu sensible et légérement oblus. - Antennes épaisses, courtes, d massue presque inscnsible.

Caractères secondaires. - Ailes jaunes, unies, jamais sablées de brun, avec un point central non argenté et une lache rose il la base des infirieurcs en dessous. - Taille au-dessous de la moyenne. - Ailes supdrieures sinuées a la côte.

RHAMNI. $\quad$ Envergure, 50 mill. - Ailes d'un| Dans toute l'Europe.| Il est extrêmement Lin. Fab. Ochs. Bdv, jaune citron, avec un point central plus Bois, prés, jardins, pen- commun. Il passe l'hi-

Hub. 442 a 444 .

Col. du Nerprun. God. pl. 2. fig. 1. Le Citron. Engx. petit aux supéricures, fauve en dessus, dant toute la belle saiferrugineux en dessous, et les nervures son. terminées par quelques petits points brunâtres.

o Un peu plus grande et d'un blane verdâtre.

une ligne latérale blanche, fondue supéricurement avec la couleur du
Chenille verte, avec miers beaux jours. tond; tête et pattes vertes. Yit sur diflérentes espèces de Nerpruns.

Chrysalide verte, avec quelques points ferrurineux sur le dos et l'enveloppe des ailes.

Cleopatra.

Ailes un peu moins angulcuses; les France méridionale, Lin. Fab. Ochs. Hub. 445-446.

God. pl. 4 D. fig. 1.

Rhamni. var. Bdy.

Le Citron de Provence. Engr. supéricures marquées chez le mâle d'une France D'après M. Boisdu- de ce lépidoptère, nous arge tache discoildale orangée.

o D'un blanc moins verdatre que val, la chenille ne diflère devons nous en rapporRhamni. $\begin{aligned} & \text { point de celle de Rham-| ter au témoignage de } \\ & \text { ni. La chrysalide a l'en- } \\ & \text { veloppe des ailes large- Boisdural, qui le } \\ & \text { ment orangée. }\end{aligned} \mid$ ver, et on le voit sou-

- MLème raison à alléguer pour l'établissement de ce genre que pour les Leucophasia. Mr. Boisdural a cru devoir changer le nom imposé a celui-ci par M. WV. Leach, parce que, dit-il, il existe un genre de Nocturnes appele Gonopicra. Comme la confusion entre ces deux nums et suttout entre ces deux genres nous parait

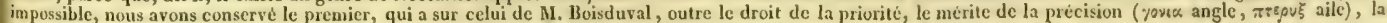
sigaification du dernier ( 


\title{
(29) Genre VII. COLIAS (COLiade).
}

\author{
(Lat, God, Ochs. Bdv.)
}

Caractères principaux. - Chenille chagrinée, subpubescente, cylindrique. - Chrysalide a ventre saillant et d partic antéricure terminée par un, pointe aiguë, droite. - Ailcs trds-arrondies. - Palpes peu comprimés, relus, leur dernier article tres-scnsible et aigu. Antennes courles, a massue visiblement distincte de la tige, qui est médiocrement épaisse.

Caractères secondaires. - Taille moyenne. - Aites d'un juune plus ou moins foncé, plus ou moins sabless de noirutire au moins ì la base; une tache rose ou ferrugineuse a la base des inférieures en dessous. - Partie antérieur'e du corselet et frange d'un rose plus ou moins vif.-Bord tcrminal des superieures, et souvent des inférieures, noir ou brunatre.

AURORA.

Fab. Ochs, God.

Hub. $544-545$.

Bdv. Icon. pl. 7. fig. $1-4$.

Dup. Suppl. pl. 6. fig. 4-5.

Le Vertumne. Engr.
Envergure, 55 mill. - Ailes d'un| Russie orientale, Si-1 orangé rif glacé de rose, les supérieures bérie, etc. lin aoat. ayant le sommet coupd carrément, et une tache costale noire cn ovale aigu, et marquée en dessous d'un point argenté, arec une bordure noire étroite coupée res ayant un point argenté géminé sur le disque, et au bord interne sculement une tache ferrugineuse.

o Plus grande, avec la base des supérieures et les inférieures très-saupoudréc de noirâtre; une large bordure noire coupée de taches jaunes qui se répètent légèrement en dessous. par les nervures. Dessous des inférieu-

M. Boisdural assigne pour patrie à cette espèce rare et mal connue avant lui, la liussie d' $\Lambda$ sie ct la Sibérie, assertion que semble confirmerson facies exotique; aussi ne la donnonsnousque parce rue presque tous les auteurs la comprennent dans les espeeces d'Europe, et qu'il est possible qu'on la trouve dans cette parlie de l'Europe qui aroisine l'Asie, et qui a été jusqu'ici peu explorée.

Cette espèce est fort Envergurc, 45 mill. - Ailcs d'un Dans toute l'Europe jaune souci, avec une large bordure Champs de luzerne et commune; mais sa cheEDab. Ochs. Bdy. God. pl. 2. secund. fig. 1. Hyale. Ilub. 429-45̃x. Le Souci. Engr. au sommet des supéricures; celles-cil En mai, aoat, septemarec une tache discoildale assez grosse, bre.

ronde et noire. Inféricures finement sa- Chenille d'un vert blées de brun, al l'exception d'un espace foncé, avec une raie labessous des supé- térale mêlée de blane et les d’un taches ovarieures souci, avec la bordure d'un jaune de jaune, et ayant sur clair que le fond, mais verdâtre; dessous des inférieures de chaque anneau un point il faut pour cela écarter cette dernière couleur, avec une séric rouge ; tête et pattes beaucoup les ailes supémarginale de taches rousses et un gros veries. Vit sur plusieurs ricures. point rond géminé argenté.

o Ayant la bordure entrocoupée de taches jaunes en dessus, et la base des espéces de trêfles, de supéricures, ainsi que les inférieures, plus sablées de brun.

Chrysalideverte, avec une ligne latérale jaune et quelques points rouges au-dessous. IYRMDONE.

\section{Ochs. Goul.}

Hub. 432-455.

Bdv. Icon. pl. 9. fig. 1-2.

Dup. Suppl. pl. 14. fig. 3-5. Le Safrané. Engr.
Un peu plus petite que l'Edusa,

Hongrie, Styrie, Rusfond des ailes d'un orangé plus vif et glacé de rose, bordure des ailes supé-let, aoot.

ricures un peu plus étroite et non divisée par les nervures; une petite tache blanchâtre au milieu du point costal des supérieures en dessous.

o Avec la bande maculaire terminale des inféricures atteignant le bord interne. et, aoot.

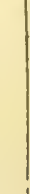




\section{G. COLIAS.}

Dup. Suppl. pl.6. fig.

L'Orange. Engr:
CunYsotaene. Oclis. God. IIub. 426-428. Bdv. Icon, pl. 9. fig. 5-4. $x-3$.
Beaucoup plus petite que l'Edusa, Hongric, Styrie, Caplus pâle, surtout à la côte des ailes su- rinthie, Russie méridiopérieures, qui sont plus arrondies; bor- nale. En juillet, noat.

dure noire plus pâle, moins découpéc et saupoudrée d'atomes jounes; point dessous, quelquefois en dessus, d'unc petite ligne blanchâtre.

ㅇ D'un jaune serin, et orangée sculement sur le disque, ayant parfois la bande maculaire des inféricures prolongice jusqu'au bord interne.

HELICE.

Hub. 440-441.

Var. o de l'Edusa. $B d v$.

God.pl. o. 4. fig. 4.

PIICOMONE.

Ochs. Bdy.

Hub. $436-457$.

God. pl. D. 4. fig. 3. Le Candide, Engr.
Ne différe de l'Edusa que par une taille plus grande et la coulcur jaune France centrale c eridionale. Dans les paile des ailes qui fait micux ressortir champs. En aoat, sepaux inférieures les atomes noirs et la ta-tembre. che discoïdale orangée. jaune pâle et verdâtre tres-aspergé de mi-côte. En juillet et brun, ayant avant le bord postérieur, aoot.

qui est plus foncé, une bande maculaire de la couleur du fond, avec un point discostal noir plus oblong, presque toujours moins gros et marqué toujours en

Elle est plus distincle que la précédente; cependant on ne peut en laire une espèce séparée arant de connaître la chenille. Nous avons d'ailleurs préféré la décrire \& la suite d'E dusa, parce qu'on la reconnaîtra mieux par les différences que dans une description séparéc.

Nous arons pris celte variété aux environs de Nogent-le-Rotrou (Eure-et-Loir); mais elle est moins rare dans le Midi et en Suisse, où on la prend principalement sur les montagnes.

Envergure, 45 mill. - Ailes d'un Montagnes alpines, à Son vol est rapide. coidal noir au milieu des supérieures. Dessous des inféricures verdatre, arec le disque plus foncé et découpant unc bande terminale plus claire, et un point central géminé d'un rose argenté.

o Plus grande, d'un blonc verdâtre, ayant la bande marginale des supéricures plus largement coupée, et celle des inféricures sans taches.

NASTES.

Bdv. Icon. pl. 8, fig. 4-5.

Dup. Suppl. pl. 15,

Plus petite ( 39 mill.). Frange et Cap nord, Islande, côte des supérieures d'un rose plus pâle Laponie.

et un peu plus étendu, point discoïdal 5 , des premieres ailes un peu plus petit et pupillé de blane en dessous. Dessous plus obscur, plus saupoudré de noirâtre; massue des antennes ayant l'extrémité d'un jaune plus clair du côté externe.

HYALE,

Envergure, 45 mill. - Ailes d'un Dans toute l'Europe,

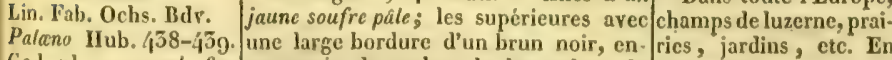
(iod. pl. 2 secund. fig.

Le Soufie. Engr.

fond, n'atteignant pas le bord interne, Chenille d'unvertvegros louté, avec trois bandes que la bordure des supoint noir; les inféricures avec une bor- longitudinales jaunes et péricures n'atteint jadure courte très-étroite, et marquées des points noirs. Vit sur mais dans toute sa larsur le disque d’un point faure géminé. la Coronille bigarrée geur le bord interne. Dessous d'un jaune plus foncé aux in- Coronilla varia).

férieures, qui sont marquées au milieu d'un point géminé argenté et d'une séric anté-terminale de taches rousses.

\& Plus grande et ayant aux ailes inféricures, en dessus, un xang anté-terminal de taches brunes peu marquées.

Envergure, 45 mill. - Ailes d'un Montagnes alpines,

PALIENO

Lin. Fab. Bdy.

| God.pl. D. 1. fig. 2. $\mid \begin{aligned} & \text { jaune verdatre, arec une bordure termi-| } \\ & \text { nale brune, large et atteigmant le bord }\end{aligned}$ aont. elle a a peu pris les mours de l'Hyale, et aime aussi ì se poser sur les fleurs. C'est alors qu'on peut la saisir facilement; elle n'est pas rare.

Sa taille est la seulc différence bien tranchéc qui la sépare de Phicomone. Si la chenille était connuc et différente, il faudrait bien en faire une espèce, mais jusque la elle est $\alpha$ peine pour nous variété de Phicomone.

Cette espécc est tréscommune. On la distinguera toujours facilement des varlétés plus pâles d'Edusa, co Nous arons trouvé une variété dont le point noir discoidal est trèspupillé en dessous, preure qu'on ne saurait invoruer ce caractere comme bien distinctif.

Elle varie beaucoup. Chez certains individus on voit sur les ailes sumais dans tour le bord interne. 
(Europome. Hub. 454435. Le Solitaire. Engr.

Philomene. Hub. 602-603

Pelidie.

Bdv. Icon. pl. 8. fig. 1-5.

Dup. Suppl. pl. 15 fig. 1-3. interne aux supéricures; courte, plus large au milieu et n'atteignant pas l'angle anal aux inférieures. Dessous de celles-ci d'un jaune sablé de noirâtre, avec un point discoidal dont le centre est d'un blanc nacré.

q D'un blanc légèrement verdâtre, avec la bordure des supérieures plus large au sommet, n'atteignant pas ic bord interne, et quelquefois marquée a l'angle apical d'une ou deux taches de la couleur du fond.

Un peu plus pâle en dessus, et sans point noir discoidal aux ailes supérieures, dessous de celles-ci avec la cóte trèsobscure. Dessous des inféricures bien plus saupoudré de noirûtre, surtout sur le disque.

Un peu plus grande que Palano, ailes moins arrondies et moins régulières, bordure brune plus étroite, point discoidal (quand il existe) plus aigu aux extrémités, surtout en dessous; point argenté du dessous des inférieures entièrement teinté de rose, et quelquefois accompagné chez lc mále d'un autre plus petit.

o Plus petite que le mále, et ayant la bordure des ailes supérieures coupée par des taches oblongues de la coulcur du fond, mais très-peu arrêtées ct même souvent dépourvues de noir du côté interne.
Laponie, Suisse.

Islande, Laponie? périeures un point noir discoidal bien marqué; chez d'autres ce point est peu sensible, et se réduit a un petit ovale evidé au centre; chez d'autres enfin il disparaît complétement ; ceux la même sont les plus communs. Cette Coliade n'est pas rare. Elle préfère les prairies parsemées de buissons de Rhododendron.

On la trouve en Suisse, dans les mêmes localités que Palano, mais elle y est beaucoup plus rare.

Cette Coliade nous a été communiquée, ainsi que la Nastes, par M. Lefebrre, qui les a reçues de Laponie; mais nous n'avons pas la certitude qu'elles y aient été trouvées. Il se pourrait que celle-ci dat par la suite former une espece; mais la Palano varie tant, que la connaissance de la chenille nous semble pour cela indispensable. Elle est encore fort rare dans les collec-

\section{(3o) Sous-tribu II. LYCRENIDI (LYCÉNIDES)*}

Caractères principaux. - Chenilles ovales, courtes et en forme de cloportes. - Chrysalides ramassées, arrondies. - Cellule discoldale des secondes ailes ouverte. - Crochets des tarses petits et d peine saillants.

Caractères secondaires. - Taille petite, ou du moins au-dessous de la moyenne. - Ailes supérieures entières, les quatre ayant lo fond bleu, fauve ou brun, toujours tris-vives en couleur.

(51) $\left\{\begin{array}{l}\text { Toutes les pattes ambulatoires dans les deux sexes. } \\ \text { Les deux pattes antérieures plus courtes et en palatine dans les mûles. }\end{array}\right.$

(32) Toutes les pattes ambulatoires dans les deux sexes.

\section{(53) Genre VIII. POLYOMMATUS (polyomate).}

(Lat. God. Dup._Lycana. Ochs.-Zephyrus. Dalm.)

Caracteres principaux. - Chenille cloporte. - Chrysalide courte et obluse aux deux bouts. - Massize des antennes en bouton allonge et presque ovoide.-Palpes de longueur moyenne.

Caractères secondaires. - Ceux de la sous-tribu**.

(a) 50 livraison de l'Jeonce de M. Boisdural,

- Cette sous-tribu était établie et caracterisee depuis long-temps. par nous sous le nom dOniscides, quand parut la 50 istle ne renferme point les Erycincs, n il l'établit aussi de son coté et lui donne celui de Lycénides. Quoique sa tribu ne réponde pas exactement a la notre, puisqu elie ne renierme point les Er àcines, avons renoncé à notre dénomination et adopté la sienne, tant pour éviter le double emploi que parce que notre premier nom existe dej a

** Il ae faut pas oublicr que le principal caractère du genre Polyommatc est d'aroir toutes les pattẹ amabulatoires dans les deux sexes. 
I. Chenilles légèrement pubescentes, convexes, arec la partie postérieure un peu dépriméc.-Chrysalides un peu oblongues. - Les quatre ailes ordinairement entières, arrondies, presque toujours bleues dans les mâles. - Dessous avec beaucoup de points ocellés, et la base des inférieures verdâtre ou bleuâtre. (Cyaniris, Dalm.; Argus, Bdv.)

II. Chenilles pubescentes, convexes, souvent un peu allongées. - Chrysalides courtes et déprimées antérieurement. Ailes inférieures ayant l'angle anal prolongé dans les mâles, et échancrées avant cet angle dans les femelles. - Fond̀ des quatre étant ordinairement d'un faure doré dans les mâles, et semé de points noirs dans les femelles. (IIeodes, Dalm.; Polyommatus, $\mathbf{B d v}$ )....
III. Chenilles pubescentes, moins ramassées que les précédentes, et ayant quelquefois la partie antérieure très-aplatie. - Chrysalides courtes, convexes en dessus, légèrement aplatie en dessous. - Ailes inférieures ayant près de l'angle anal un prolongement souvent très-long et en forme de queue. (Aurolis, Dalm.). . . . . . . . . . .

\section{(35) Division I. CYANIRIS, Dalm. ARGUS, Bdv.}

Chenilles ligirement pubescentes, convexes, avcc la partic postérieure un pcu déprimée. - Chrysalides un peu oblongues. - Les quatre ailes ordinairement entieres, arrondies, presque toujours bleues dans les mäles. - Dessous avec beaucoup de points ocelles, et la base des inférieures rerdâtre ou bleuâtre.

$$
\text { (Les Azurins. Lat. God.-Polyophthalmi. Ochs.). . (36) }
$$

(36) (Dessous des inférieures avec une série anté-marginale de taches faures plus ou moins apparentes: . (5y)

(36) Point de série anté-marginale de taches faures lunulées sous les inférieures. . . . . . . (49)

\section{(37) Dessous des ailes inférieures offrant une série de taches fauves lunulées plus ou moins sensibles. (38)}

$\left\{\begin{array}{c}\text { Ailes inférieures ayant en dessous, d la moitié de leur largeur et près du bord terminal, un espace blanchâtre assez large, } \\ \text { mais court. } \\ \text { Point d'espace blanchâtre au bord marginal des inférieures en dessous. } \\ \text { L'espace blanchâtre plus rapproché de l'angle anal et formant deux gros points souvent marqués d'une tache noire, qui } \\ \text { dépassent une série marginale de taches blanches. } \\ \text { L'espace blanchâtre commençant à former une bande, mais qui ne se prolonge pas au-delà de la lunule discoî̉ale. }\end{array}\right.$

(59) Ailes inferieures ayant en dessous, il la moitie de leur largeur et prìs du bord terminal, un espace blanchûtre assez large, mais court.

$\left\{\begin{array}{l}\text { Frange entrecoupée.. } \\ \text { Frange non entrecoupée. }: \cdots(41)\end{array}\right.$

\section{(41) Frange entrecoupie.}

CORYDON. $\mid$ Envergure, 34 mill. - Ailes d'un| Dans presque toute $\mid$ On rencontre quelFab. Ochs. Bdv. Hub. bleu argenté luisant, avec une bordure l'Europe, dans les bois quefois des femelles qui 286 ì 288. noire large, ocellée aux inférieures. Des- secs et les lieux pierrcux sont entièrement bleucs Poly. Coridon. God. pl. sous des supérieures blanchatre, avec et incultes, les prairies, en dessus. Nous verrons 11 sec. et tert. fig. 1. une rangée marginale de taches ocellées etc. Fin juillet et cou- dans beaucoup d'autres L'Argus bleu nacré. de la même couleur. Dessous des infé--rant d'aoat. Azurins (Alexis, AdoEngr. $\quad$ rieures brunâtre, a base verdatre, avec Chenille d'un vert nis, Argus, etc.) le Var. $\$ 742$. Hub. (Voy. la tache discoildale toute blanche et les foncé, ayant sur le dos même accident se rela note). lunules d'un faure vif. deux rangées de crêtes produire. Les couleurs ㅇ Brune, arec une lunule discoidale saillantes d'un beau jau- de ce Polyommate sont noire sur les supérieures, et les taches ne, séparéesparle sillon fort solides; aussi en ocellées des intérieures marquées de dorsal, mais nulles sur troure-t-on encore de fauve. Dessous d'un brun roux vif, sur- les trois derniers an-très-frais dans le coutout aux inférieures, arce les points neaux; côtés saillants rant de septembre. II gros; bien entourés de blanc, la rangée et entièrement bordés n'est pas rare, mais ses terminale marquée de fauve, même aux d'une ligne jaune au-localités sont ordinairesupérieures, et un très-petit trait noir dessus de laquelle sont ment assez resserrées. dans la tache discoîdale des inférieures. les stigmates, qui sont On distinguera toujours noirs et a peine visibles. sa chenille de celle d' $A$ Tête noire; pattes de la donis, à laquelle elle conleur du fond. Se ressemble extrêmement trouve en mai et juin par la petitesse de ses sur les Trifolium, Lolus, , stigmates ct sa couleur. 
Adunis. var. God. Bdy
Pour crécr cette espèce, Hubner représenté un mâle qui a sur les ailes les ailes France, en vírons de $\mathbf{P}$ noirs le rencontre très-fréquemment; puis une femelle dont le bleu azuré a envahí toute la surface en dessus, de sorte qu'elle est à peu près du ton du mále, avec une séric marginale de lunules fauves aux inféricurcs. Elle est plus rare et habite principalement l'ouest et le midi. premier; les stigmates| très-apparents, noirs, et İ tête de cette même couleur. On la trouve en mai sur les 'Trifolium,

Lotus, Hippocrepis, etc. Chrysalide brune ou roussâtre. $\boldsymbol{\Lambda}$ la surface de la terre.

Il cst très-certain que ce n'est qu'une variéte d'Adonis, comme on en rencontre beaucoup d'analogues dans cette division. Nous l'avons prise dans les environs de Blois, d la forêt de llussy.

\section{(42) Frange non entrecoupce.}

DORYLAS. Envergure, 35 mill. - Ailes d'un| Montagnes alpines, D'aprèsla figure donFab. Ochs. God. II bb. bleu d'azur, avec une petite bordure et en juillet; prairies hu- née par H. Duponchel, 289-291.

Dup. Suppl. pl. 12. fig. des supérieures sans points à la base; mai et juin. 1-4.

des supérieures sans points à la base
dessous des inféricures d'un brun clair,

Bdr. Icon. pl. 14. fig. à base verdâtre, avec des points ocellés,

$1-3$

L'A zure. Engr.

le bord marginal argement blanchâtre, une lunule discoïdale toute blanche et une séric de taches fauves sagittées, appuyées presque toutes sur des points noirs très-petits.

ㅇ Brunc, avec une série marginale de taches faures. Dessous d'un brun plus foncé, arec les taches faures plus grandes, plus vives, les points noirs marginaux plus gros, et un trait noir dans la lunule discoïdale des inféricures.

Gorcus

Hub. 688-689.

ALEXIS,

Envergure, 3.s mill. - Ailes d'un 29: 1 .

Bdv. God. pl. 11 sec. fig. 3.

L'irgus bleu et $l^{\prime}$, $\mathrm{S} r$ gusblcutiolet. Engr. blcu violâtre, soycux, très-finement bor- dans les prés, les jar- des Polyommates. On dées de noir. Dessous d'un cendré clair, dins, les champs de lu- rencontre sourent des avec des points ocellés, dont deux ou zerne, ete, pendant femelles dont le dessus trois d la base des supéricures, et unc toute la belle saison. des ailes est entièrement rangéc de taches faures triangulaires Chenille verte, avec saupoudré de violet, appuyée sur des points noirs. q Brune, plus ou moins saupoudréc sur le raisseau dorsal, correspondante de l' $\boldsymbol{A}$ de violet, arec des taches terminales fatı- et quelquefois deux au-donis. On le prend facileres en dessus, et le dessous d'un cendré tres lignes semblables ment dans toute sa frairoussâtre. lyommate, il paraitrait Tu'cle a quelquefois un point ocellé is la base superieures, preure que ce caractere ne saumitetre invoque comme it n'existe point dans toutes les femelles que nous avons vues.

Dans cette espice, comme dans l'Adonis et plusicurs autres, les points ocellés des ailes supéricures sont quelquefois d'une grosseur démesurée.

Ilne mérite pas même le nom de variété.

sur le dos; tête et pattes cheur. Nous ayons pris noires. Vit en mai et une variété mâle quia au juillet sur la luzerne bord marginal des infé(Merlicago saticu), la rieures, en dessus, une bugrane (Ononis spino-série de points noirs, sa), le fraisier (Fragaria comme le Ceronus nuile resca), l'Astragalus gly-d'Hubner.

ryphyllos, etc.

Chrysalide d'un gris roussâtre. A la surface de la terre. 


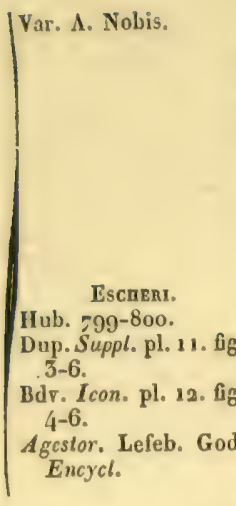

FROS.

Ochs. Bdr. Icon 1. . fig. 4-6.

Dup. Suppl. pl. 14. fig. 5-6.

Tithonus. God. Hub. 555-556.
Elle ne differe de l'espèce que par l'absence des points ocellés de la base des supéricures en dessous. De sorte qu'elle n'en a point arant la lunule discoidale.

Il est plus grand; les lunules faures du dessous sont plus pozere, Suisse. En juin lés de la base des supérieures en dessous manquent comme dans notre variété $\mathbf{A}$. d. riéte \& Analogue \&̀ la précédente.

Environs de Paris. damment les deux sexes de cette variété dans une petite localité près de Châteaudun. Elle fait le passage d'Alexis à Escheri, et proure que ce caractère invoqué pour les diviser n'est pas constant.

La femelle est plus rare que le mâle. La connaissance de la chenille de ce Polyommate, qui n'a, dit-on, qu'une génération par an, est indispensable pour le séparer de l'Alexis. On le trouve en Suisse, mais il y est rare. Dans la Lozère, au contraire, il est assez commun.

Envergure, 51 mill. - Ailes un peu

Alpes du Tyrol, de la

Il a été très-rare et Suisse, Mont-Cenis, commence d le devenir oblongues, d'un bleu argente brillant, Suisse, Mont - Cenis, mité des nervures noires. Dessous d'un Gap. En aoat. gris cendré, arec des points ocellés, dont un ou deux à la base des supérieures, et des rangées marginales de taches noirâtres et faures disposées comme dans Alexis.

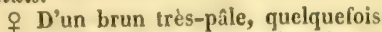
sablée de bleu, avec une lunule noire et une rangée marginale de taches fauves en dessus. un peu moins. Nos in-
dividus nous viennent
de $\mathbf{M}$. Anderegg. Le ton
verdâtre et argenté de
son bleu le sépare faci-
lement de Dorylas et
d'Alexis.

Point d'espace blanchatre au bord marginul des inférieures en dessous.

Frange non entrecoupée. . . . (45)

Frange entrecoupée. . . . . (46)

\section{(45) Frange non entrecoupe.}

ICARIUS. $\quad$ Envergure, 54 mill.-Ailes d'un bleu Suède, Hongrie, La- Il n'est pas trés•comOchs. God. Bdv. Icon. presque azuré luisant, arec une bordure ponie, Pyrénées, alpes mun. L'Agathon de pl. 12. fig. 1-3. très-étroite et l'extrémité des nervures du Piémont, Barèges, God., que nous avons Dup. Suppl. pl. 11. fig. noires, et sourent un rang de points de Mont-Cenis. En juillet vu dans sa collection, $1=2$. cette couleur au bord marginal des infé- et aoct.

A gathon. God. Encycl. rieures. Dessous d'un gris cendré uni,

Amandus. Hub. 752 - avec des points ocellés assez petits, mais -55 .

Var. accid.? 285-285. série marginale do taches d'un fauve pále, visible seulement aux inférieures, qui sont trés-arrondies.

o Brune, arec un arc noir discoidal aux supérieures, et des lunules faures terminales aux inférieures et quelquefois aux supérieures; dessous d'un gris plus foncé, arec ces mêmes lunules yisiblets.s n'en differe que par un bleu un peu plus argenté, la bordure noire un peu plus large et les lunules faures plus isolées en dessous. Ces différences ne peuvent pas même constituer une variété, et ne sont que celles qui s'observent sourent d'un individu it l'autre. Il parait, d'après Dalman et la figure 284 d'Hubner, que la femelle est quelquefois saupoudrie de bleu i| 
Lin. Fab. Ochs. Hub. $3,6-3,8$. Bdv. Icon.

Calliopis*

L'Argus bleu? (80 $\left.\mathrm{f}_{4}\right)$.

Engr.
God. pl. 11 tert. fig. 4

la base et sur les ailes inféricures.

Nola. Il ne faut pas oublier que le principal caractere de cette espèce est celui indiqué par la division.

Envergure, 3o mill. - Ailes d'un bleu Dans toute l'Burope, Cette espece est exviolet foncé, avec une bordure noire or- dans les bois secs et trêmement difficile d̀ dinairement assez large et la frangeblan- couverts de bruyeres. distinguer de la suiche. Dessous cendré, avec des points ccr- En juin et aout. vante, et tous les caracnés de blane sale (mais point à la base Chenille d'un vert tères employés à cet des supérieures), et une série anté-mar- obscur, avec une ligne effet par lesauteurs sont ginale de taches fouves se confondant ferrugineuse sur le vais- fautifs ou peu constants. entre elles, et bordées antérieurement seau dorsal, et d'autres Il existe peu d'espèces par des chevrons noirs surmontés de ta- obliques et bordées de d'ailleursqui varientauches sagittées peu sensibles d'un blanc blanchâtre sur les câtés; tant, quoique les variésale, et extérieurement par des points tête et pattes écaillcu- tés soient peutranchées. noirs sablés de vert métallique.

ses, noires. Vit en mai Nous arouons donc ici

o Brune, presque toujours saupou-sur le Melilotus officina-notre impuissance à drée de bleu à la base, avec des lunules lis, différentes espèces donner de bons caracfauves (manquant souvent aux premiè- de Genista, etc., etc. tères distinctits - entre res ailes, et quelquefois, mais rarement, Elle préfère la feuille clles, et cette opinion aux secondes, ou elles reposent sur des aux fleurs. est le fruit de longues points noirs) et la frange sale. Dessous Chrysalide un peu al- et minutieuses compaplus foncé et plus vif en dessins que le longée, verdâtre ou bru- raisons, tant sur les aumale.

natre, arec l'enveloppe teurs que sur la nature. des ailes plus foncée. Nous dirons seulement que l'Argus est presque toujours plus grand, et que la couleur blanche qui cerne les points noirs et qui surmonte la bande faure des inférieures est ordinairement d'un ton plus sale et moins vif; que la série de points ocellés des premiéres ailes est mieux alignée inférieurement, et que les antennes des miles ont ordinairement Ia massue d'un ton plus foncé. Quant à l'époque d'apparition, elle varic suivant les localités.

On rencontre parfois, comme dans Adonis, Alcxis, etc., des femelles entièrement bleues, et le ton du dessous est quelquefois plus clair, arec les points plus petits. La réunion de ces accidents constitue la rariété figurée par M. Boisduval, Icones, pl. 15, fig. 4-5, mais ils s'observent aussi isolément.

Linvergure, 25 mill. - Ailes d'un bleu Dans toute l'Europe, Les points oculaires

EGON.

Ochs. Bdv. II ub. 3:3-violet fonce, avec une bordure assez lar-dans les bois sees sur sont ordinairement plus 3,5 . ge noire et la frange blanche. Dessous la bruyère. lin juin et gros a proportion que cendré, arec de gros points noirs cernés juillet.

dans l'Argus, mais ce de blanc assez vif (mais point à la base Chenille d'un gris/earactère n'est point

- Les personnes qui adopteront le genre Argus de M. Boisduval devront, pous éviter la répétition du mot, adopter aussi le nom de Calliopis pour cette cspetcr. 
(des supérieures); unc série de taches|bleuâtre, arec une ligne/constant. Les points faures fondues ensemble, et bordée inté- dorsale noire interrom- ocellés inférieurs de la ricurement par des ares noirs, puis par pue et une bande rous- série des premières ailes des taches sagittées d'un blanc assez vif, sâtre sur les côtés; tête rentrent aussi davantage et extéricurement par de gros points et pattes écailleuses noi-en dedans que dans Arnoirs quelquefois sablés de vert métal- res. Se trouve en mai sur gus. Ce dernier caraclique.

le baguenaudier (Colutea tère est constant sur tous

o Brune, avec des taches anté-mar- arborca) et sur le $G_{e}$ - les individus que nous ginales faures assez grandes. Dessous nista scoparia. avons observés, mais plus foncé et plus vif en dessins que le Chrysalide d'un gris nous n'osons assurer

(FaliLTER. ali.? Ochs.? Encycl.

God, Hist, nat. pl, 26 z. 0 g. 5-4.

Cyparissus. Hub. 654$65 \%$.

L'Argus bleu lurquin. lingr.

Optilete.

III). 510.512 . male, et frange plus sale.
Envergure, 27 mill. - Ailes d'un riobrun un peu verdâtre, avec l'enveloppe des ailes plus foncée.
Nous possedons. variété mîle qui diflère de l'espèce en ce que la bordure noire est extrêmement large, et en ce qu'il y a une trè-petile lunule noire sur le disque des supérieures en dessus.

Suisse, cantons des Quoique connu deGrisons, près de Coire puis long-temps, ce Ponoire. Dessous d'un cendré un peu obs- et d'Uri, près de Rẻalp; lyommate est assez rare

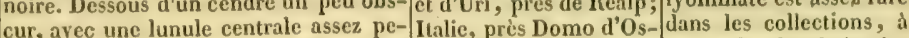
tite, suivie aux supérieures d'un rang sola, Hongric, Suède, cause des localités cir-

de sept i huit points noirs ocellés, et aux Piemont. En juillet et inféricures d'un rang très-pexueux de aoat. huit à neuf; base des mêmes ailes marquée de deux points ocellés, les quatre ayant un double rang anté-terminal de taches plus foncées, dont les trois ou quatre dernières des inférieures marquées entre elles d'une tache faure, et la dernière et l'anté-pénultième de la rangée inférieure saupoudrée de bleu métallique.

o D'un brun noir, avec la base un peu bleue et la frange très-blanche; inféricures marquées au bord terminal d'une petite ligne blanche interrompue.

Plus grand; d'un bleu assez clair. Dessous d'un cendré très-foncé, avec une lunule centrale grosse, suivie aux supéricures de cing points, et aux inférieures d'un rang courbe de six point ocellés; un seul point d la base des inféricures; double rangée de taches terminales plus grosses aux inférieures, l'anté-pinultième seulement marquéc de fiuve.

of Largement bleue, avec une tache lauve aux inféricures correspondant at celle du dessous.
Allemagne? Son rolest assez liapide. Nous ne pouvons affirmer que l'Optilète de Fab, et d'Ochs. soit bien le nôtre, quoique nous le présumions.

Nous n'avons point vu cette variétí en nature, nous ne pourons done certifier que ces differences qui la séparent du Cyparissus d'Hub. (qui est notrc Optilte et celui de God.) soient bien constantes.

\section{(46) Frange entrecoupéc.}

I3ATTUS. | Envergure, 28 mill.-Ailes d'un noir Allemagne, Russie, Il aime à voltiger auOcls. Bdv. Ifub. 328- brunâtre, arec le disque des supéricu-Piémont, Italic, Suisse, tour des buissons. En $\begin{array}{lll}\text { 350. 801-802. } & \text { res saupoudré de bleu-violet et marqué midi de la France, dans } & \text { Suisse, c'est dans les } \\ \text { d'une grosse lunule centrale noire; infé- les bois fourrés. En cantons les plus méri- } \\ \text { Telephii. Fab. }\end{array}$ Telephii. Fab. Telephii. Fab.
Poly. de l'orpin (Tele- d'une grosse lunule centrale noire; infe- les bo ares ayant au bord terminal une série juillet.

dionaux et particulièrephii). God. pl. 25 x. d'urceaux d'un bleu violet. Dessous d'un Chenille d'un vert de ment dans celui du 'T'esfig. - -8. Ulanc terne, avec une multitude de taches mer, avec une ligne vio- sin qu'il faut le chercher.

L'Argus brun. lingr. très grosses non ocellèes, dont plusicurs lâtre sur le dos. Se trou-

s.j sont carrées; inféricures avec un cordon ve cn juillet sur le Sedum presque discoidal de taches faures, bor-|Telephium. 
dées inférieurement par des points, su- Chrysalide obtuse, péricurement par des arcs, noirs. courte, verdâtre, mouo Beaucoup plus grande et n'ayant chetéc de brun. pas de bleu en dessus.

IIITAS. Fals, Ochs, Bdr. II ub. $325-527$,

God. pl. 11 tert. et 11 sec, fig. 5.

L'Aigus licu riolet. lingr.

Payortes. [Jub. $6-0-673$.
Envergure, 22 mill. - - iles d'un bleucondré-violatre, pâle, les quatre ayant une lunule discoirlale noive; inféricures avec

une série marginale te points noirs. Dessons d'un gris cendre avec de gros points légèrement ocellés; inféricures it base bleuatre et ayant, assez loin du bord terminal, une sćrie de taches fauves, arrondies, renfermées chacune entre deux points noirs.

\& Plus grande, d'un biun noirâtre clair, avec un peu de violattre à la base et les points marginaux des inférieures cernés de blanchâtre.

Un peu plus foncé, avec les nervures plus narquéces et les lunules centrales plus grosses, bordure plus large et fonduc arec la couleur du fond; bande fanre alu dessous des inferieures en totalite ou en partie obliterese.

f. Analogue.
Dans toute la France. En mai et ant.
Il n'est pas trís-commun aux environs de P'aris. Il aime à voltiger sur les fleurs de thym et de serpolet: nous le prenions autrefois abondamment près de Cliartres; il est plus commun dans le midi.

C'est à peine une rarićté, ct, ì l'oblitération près de la lsande fauve, les $\boldsymbol{H}_{\text {ylas }}$ un peu passís présentent les nêmes caractères. Nousn'avons ru en nature que le mâtle; mais Hubner figure aussi la femelle.

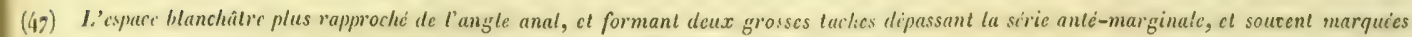
"'un point noir.

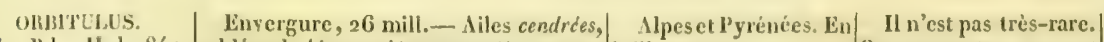
Ochs. Bilv. II ub. 841. sablées de blcu-verdätre très-pâle, argentej juillet et aoot. Meleager. Hub. 522- avec une lunule noire, cernée de blan$525 \mathrm{ct}-6 \mathbf{1}_{-}-6_{2}$. chatre; les supérieures aiguës au somPoly. Orbilulus. God. met, les inférieures arec une série antépl. 25 r. fig. 3-4. marginale de points noirs cernés de blanchatre. Dessous des supéricures d'un cendré clair avec des points noirs ocellés. Dessous des inférieures brunâtre, avec plusicurs taches blanches dont une centrale cordiforme, les autres marginales et marquées presque toujours de points ou eherrons noirs et de deux taches fiuves pris de l'angle anal.

을 D'un brun noir, arec les lunules centrales moins visibles, surtout aux inférieures.

Souilo. 7.8.
Un peu plus petit (22 mill.). Ailes supérieures un peu plus arrondies, dessous
plus sombre (comme dans toutes les espéces boréales).

\& Arec la lunule centrale bien sensible sur les quatre ailes, qui sont du. même ton que celles du mâle, et doni les supérieures sont marquées de deux séries anté-terminales de taches blanchâtres cunéiformes.
Cap-Nord. On rencontre sourent des individus dont les taches blanches des secondes ailes en-dessous ne sont marquées d'aucun point noir ni fauye. Telle est la variété $522-$ 525 d'Hubner.

Le vol dece Polyommate est rapide, mais it sc pose souvent et est alors facile à approcher.

Nous n'ayous vu en nature que le maile, et nous devons dire qu'il nous a paru à peine variété d'Orbitulus, mais si sa femelle est toujour's aussi tranchée que 3. Bdy. l'indique, il est possible qu'il doire former une espèce. Cependant, l'Orbitulus rarie tellement que nous avons cru devoir attendre pour cela la découverte de la chenille. On te trouve aussi cn Sibéfie et au Iabrador. 
(48) Lespace btanchätre commenfant à former unc bande, mais qui ne se prolonge pas au-ileld de la cellule discoildale.

Donzelif. | Envergure, 29 mill. - Ailes d'un Départenient des Bas-| Cette jolie espice est Bdiv. Icon. pl. 15. fig. brun cendré, avec le disque d'un bleu- ses-Alpes, environs de encore rare. M. Bdv. fi$1-3$.

Dup. Suppl. pl. 8. fig. $1-3$.

Hแb. $955-957$.

(Dessous mal figuri.)

verdâtre argenté et un are discoîdal Digne et d'Alloz; I Ia - rnure la femelle avec la noir. Dessous des supérieures d'un cen- tes-Alpes, environs de bandelette blanche du dré jaunâtre avec de petits points ocellés, Briançon, dans les bois dessous dépassant la lumais point a la base. Dessous des infé- de meliezes, $\Lambda$ p pes clu nule, mais il n'en paric ricures avec des points semblables et Vallais. En juillet. point dans sa descriptraversé par une bande blanche qui ra s'appuyer sur un rang court de chevrons lauves, étroits et à peine, sensibles.

o Entièrement brune.

tion. Pour nous, les trois individus que nous arons vus avaient cette bande comme dans la figure de M. Dup., qui est très-bonnc. C'est ce qui nous engage at le laisser dans cette section.

EUMEDON. Ochs. Bdr. Hub. 501302 et 700-701. God. pl. 25 x. fig. 1-2. L'Euméton. Engr.

Emergure, 5i mill. - Ailes d'un Alpes, Pyrénces, PieCette espice n'est pas brun noiratre, les supérieures avec une mont et Allemagne, mi- rare. Nous l'avons prise petite lunule discoîdale plus foncéc. di de la France, ete. En plusicurs lois dans les Dessous d'un cendré grisâtre ou jamnâ- juin, juillet et nout. prairies élevées des Pytre, arec des points ocellés; lunule des supérieures trés-grosse, inférieures arec les points ocellés bien alignés et un rang anté-marginal plus ou moins visible de petites taches faures, surmontées d'un chevron noir et reposant sur un point de même couleur; base largement verdâtice.

o Semblable, mais ayant ì l'angle interne des inférieures et quelquefois même des supérieures en dessus, un rang de taches fuures plus ou moins nombreuses. Celles de dessous mieux marquées. rénées aux environs $\mathrm{d}^{\prime} \Lambda \mathrm{x}$. II paraît qu'on rencontre des femelles qui n'ont point de taches fauves en dessus et qu'elles sont bien plus communes en Suède.

(49) Point de série anti-marginale de taches fauves lunulées sous les inféricurcs.

(Des faisceaux de poils sur le disque des ailes supérieures, ou une bandelette blanche longitudinale sous les inférieures, (5o) remontant presque jusqu'it lit base. - Diles inférieures toujours un peu, quelinefois fiortement, échamerées prés de l'angle

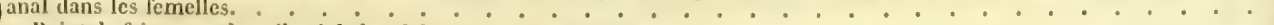
P'oint de faisceaux de poils ni de bandelette, ailes très-entières. : . . . . . . . . . . . . . . . . . (5

(51) Des faisceaux de poils sur le disque des supéricurcs, ou une bandelctle blanche longriludinale sous les inféricures, remontant presque ju qu' a la base. - Ailes inférieures toujour's un peu, quelquefois fortement, échancries prds de l'angle anal dans les femelles.

DAlion. $\mid$ Envergure, 35 mill. - Liles entières| Alpes, Pyrénées, Cé-| Cette belle espèce.est Fab. Ochs. Bilv. Hub. d'un bleu-verdatre-pâle argenté trés- vennes, Lozère, Alle-lrès-commune. Nous $275-277$.

God. pl. 2. x. fig. 5-6. se rétrécissante large bordure brune, magne, etc.

L'Argus bleu a bandes frange blanche. Dessous d'un cendré jaunâtre, aree une raie élevées des Pyrénées et brunes ct une ligne jaunátre; supérieures avec une lunule dorsale plus foncée, dans les montagnes du blanche. Engre. centrale et une série de gros points puis une autre latérale grand duché de Bade
ocellés, inférieures avec une série sem- semblable bordée de qui avoisinent le Rhin. blable et une bandelette blanche, bien blanc, ot suivie d'une marquie et constante.

o D'un brun-noir saupoudré de bleu d'un jaune paille au-desi la base, frange grisâtre et dessous sus des pattes. Setrouve plus roussâtre. i la fin de mai sur les
Hedysarum onobrychis et
supinus.
Chrysalide oblongue,
cylindrique ct obtuse
auxdeuxbouts, d'unjau-
ne ochracé ou verdfitre. 


\section{G. POLYOMMATUS.}

I) OLUS.

Hub. $7955-796$. Var. accid. $828-829$. Bvd. Icon. pl. 15 , fig. 6-8.

Dup. Suppl. pl. 10. fig. $5-5$.

Lefelorei, God, Enoycl. et Tabl. mithod.

MUP'ENTIT. Bdr. pl. $16 . \mathrm{fig}$. 4-6. $1-2$.

IIub. 958-960. Dup. Suppl. pl. 10. fig.

Linvergure, 35 mill. - Ailes d'un| Département du Var toyant, avec un liseré étroit et l' cha- et de la Lozére, sur les fort rare dans les collecmité , a mité des nervures bruns; supérieures la fin de juin au com- du maintenant. M. Duaynt sur le disque un duvet colonneus mencement d'aoat. ponchel l'a pris abonpais et brunâtre; dessous d'un cendré Chenille verte, arec damment danslaLozere, jaunâtre, surtout aux inférieures, arecle dos caréné et bordé d̀ un quart de lieue de une lunule centrale et un rang de decharque cotcide taches Florac.

points ocellés; les inférieures ayant de jaunaties un peu oblilégères lunules terminales blanchátres et ques, séparées par des sourcnt une bandelette blanche longitu- lignes vertes plus mardinale.

quées, côtés violitires

$q$ Brune, sans faisceaux de poils et terminés par une ligne avec le dessous plus foncé et roussatre. jaunatre. On la trouve en mai sur le sainfoin (Onobrychis saticu). Chrysalide obtuse, d'un brun roussître, ou verte ponctuée finement et irrégulièrement de noir.

Envergure, 55 mill, - Ailes d'un Département des liasayant la frange $d^{\prime} u n$ brun sale et un du-zère. En juin ct juillet vet cotonnenx Jrun très-étendu, surtout au bord interne; inférieures légèrement échancrées à l'angle anal. Dessous d'un blanc-jaunatre sale, les supéricures avec un are central et une bande arquice de points ocellés assez gros; les inféricuresarec une bande arquée de points plus petils et dont le deuxième (a partir de la côte) nul ou presque nul, el une baniletetle blanclie irds-bien marquée et constante; point ou sculement quelques traces de lunules ante-marginules plus foncies.

q Sans faisccaux de poils, arec les nervures plus foncées et ayant une lumule centrale noire sur les supérieures, inféricures un peu sinuées, arec la frange blanchatre. Dessous plus foncé, avec les points ocellés des supérieures plus gros.

ADMFTUS

Ochs. God. IIub, 507509 .

Dup. Suppl. pl. 10. fig. (i-7 of (et non o $)$. L'Argus capucin. Engr. égal des deux côtés; les inféricures lé- juits.

gìrement échancrées près de l'angle anal. Dessous d'un cendré jaunâtre, les supéticures avec une bande arquée de points ocellés et un are central plus gros qu'aux inférieures, celles-ci avec une brande arquée de points dont le deuxicme (a partir de la côte) presque aussi marqué que les autres et un double rang de lunules brunes antd-marginales; point de bandcletle blanche.

o Sans faisceaux de poils et ayant une Junule centrale noire sur les supérieures, les inférieures un peu sinuées, avec une rangce ante-marginale de linules |ratces. Dessous plus jaunâtre, it points
On apercoit quelquefois sous les ailes supdrieures de la femelle une trace de handelette blanche longitudinale comme aux inférieures. Ce Polyommate, qui aura sans doutc été confondu long-temps arec le suirant, est très-commun dans les Basses-Alpes. Sa femelle se distinguera: $1^{\circ}$ de celle de Damon par les sinuosités de ses secondes ailes, et l'absence des atomes bleus i) la base; $2^{\circ}$ de celle d'Adnetus par l'absence des lamules faures: 5 de celle de Dolus, avec laquelle elle a les plus grands rapports, par la frangre des superieures, qui est brune, tandis qu'elle est blanchatre dans le premier.

On remarque quelhic; environs de Tou- quefois dans les mâles,
? et de Lyon? lin et fort sotivent dans les lemelles, des traces de bandelette blanche sous les inféricures, mais jamais cette bande n'est prononcée. La femelle se distinguera facilement des espèces roisines par ses lunules faures. Elle ferait à cause de ce dernier caractère exception at sa division (49); mais les lunules fauyes, quoique bien marquées en-dessus des ailes inférieures, le sont bien rarement en des- 
plus gros et it taches anté-marginales teintées de faureaux supéricureset quelquefois inférieures.

MELEAGER : $\quad$ Envergure, 40 mill.-Ailes d'un bleu x. figr. 1-4.
Dıphnis. Ochs. II uh. dure étroite et l'extrémité des nervures nes, Lozère, etc. Dans de ciel argenté, chatoyant, avec une bor$280-282$. blanchüre, avec des points ocellés; les juillet.

L'Argus bleu pále (84 inféricures ayant une série anté-margiA. в.). nale de taches chevronnées à peine disEt l'Argus bleu dicou-tinctes. pé (8i A. B.). Eingr. poudré sur le disque de bleu brillant coupé par les nervures. Supéricures avec une lunule discoildale plus foncée; inférieures fortement dentées, avec une rangée marginale de taches brunes ocellées, surmontées de eherrons blanes. Dessuus d'un gris roux, avec les mêmes dessins que le mâle, mais bien mieux marqués.

Cixves.

Hub. $850-851$.

Ce Polyommate, que nous n'arons vu que dans IIubner, différerait principalement du Meleager femelle en ce que les inférieures ne sont point dentées, et en ce que le dessous des supérieures et le bord marginal des inférieures sont blanchîtres et marqués d'une séric de lunules fauves. sous, et jamais d'ure manière prononeće.

Cette espéce est une des plus grandes et des plus belles du genre. 1. Duponchel, qui l'a prise trés-abondamment clans une localité remplie d'Orobus niger, présume que sa chenille vit sur cette plante. Les femelles prises en Ilongrie sont plus petites et entièrement brunes.

Godart remarque, au sujet de ce Polyommate, que les femelles sont plus petites que les mâles. Cette particularité s'observe anssi dans les quatre espèces précédentes. Dans les Azurins en général les femelles dépassent rarement la taille de leurs mâles, et l'égalent le plus souvent.

Nousnesaurionsnous prononeer sur cette espéce remarquable avant de l'avoir vue en nature. Il est possible qu'elle ne soit qu'une variété accidentelle du Meléager. Hubner ne donne pas le male.

$$
\text { Pcint de faisceaux de poils ni de bandelctle, wiles très-enticircs. }
$$

$$
\left\{\begin{array}{l}
\text { Pas de points noir's sur la surface supérieure des ailes. } \\
\text { Une bande arquée de points noirs sur les ailes supéricures, au moins dans l'un des deux sexes. . . . . . }
\end{array}\right.
$$

\section{(54) Pas de points noirs sur la surface supiricure des ailes.}

10Las. $\quad$ Envergure, 42 mill.-Ailes d'un bleu-| IIongrie, Dalmatie,| Cette belle et rare es-|

Ochs. Bdv. Icon. pl. 11. violatre luisant, avee un liseré noir et Italie; environs de Tou- pèce est anssi une des fig. $1-5$.

la frange blanche. Dessous cendré clair, lon et de Saint-Maximin plus grandes du genre.

Dup. Suppt. pl. 7. fig. avec une petite ligne centrale et des (département du Var). $4-6$.

Jolans, Hub. 8,9-882. points noirs cernés de blanc (ces der- In juin et juillet.

niers plus gros aux supérieures), et une Chenille d'un brun rangée marginale de lunules blanchâ- café ou verdâtre, avec tres marquées chacune d'un point noi- une ligne dorsale noire, râtre, ces points plus apparents à l'an- et une large bande latéfrle anal. rale plus clairc. Tête bru-

o b'un brun noir avec le disque bleu- ne. Vit dans les capsules et trois ou quatre des taches ocellées du Colutea arborescens. anté-terminales apparentes en dessus Chrysalide arrondie, près de l'angrle anal des inféricures. grisâtre, ponctuéc de

ACIS. Enverrure, 28 mill- - Ailes d'un bleu- noir. ir.

Il est tres-commun, Ochs. Bdv. God. pl. 1 riolet foncé, arec un petit trait discoildal, tie de l'Europe, prés et mais un ne le troure|

- La plupart des auteurs ont bien remarqué les faisceaux de poils sur le disque des supérieures dans Admelus, Dotus, ctc., et aucun ne les a vus dans Melcagth lls y existent pourtant, sourent moins prononces, il est rai, que dans ces especes, mais toujours sensibles. 
sec. fig. F. el 1 q quart.|les neroures et une bordure ctroite mais/clairières des bois hu-|abondamment que dans lig. 4 . Argiolus. Fab. $269-271$. fondue dans la conleur du fond, noirs, et mides. En mai et juillet. Le Demi-Argus ( D. C. D). Engr.

Hub. la frange blanche. Dessous d'un cendré obscur, arec une rangée de points ocellés

$(88$ A. et une lunule centrale; séric de points courbe et sinuce aux premières ailes, qui n'ont pas de point basilaire.

of Enticrement d'un brun noir, avec la frange d'un blanc sale, excepté au sommet iles supérieures.
Skmius. HIub. 85 ı-85午. Bdr. Icon. pl. 17. fig. I-5.

Saporta. Dup. Suppl. pl. 9. fig. 5-7.
Envergure, 25 mill.-_ liles d'un bleuviolet foncé, arec un liseré noir étroit et bien arrête, et la frange blanche. Des- d'Hières. Dans les lieu
sous d'un gris de perle, arec un rang de secs, sur les fleurs d points ocellés et une lunule centrale; sainfoin. En mai.

série de points sles premières ailes droite, it la réserve de celui du sommet. o D'un brun noir, arec la base saupoutrce cle bleu-violet et la tirange trèsblanche partout.
ALSUS.

Fab. Ochs. Bdy.

IIub, $278-279$.

God. pl. $26 \mathrm{z}, \mathrm{fig} .5-6$. Le Demi-Argus ( 88 E. F.). Engr?
ARGIOLUS.

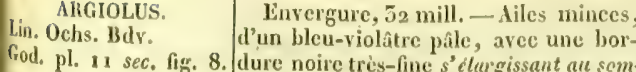

et $x$ is sec. fig.$$
\text { tcis. }
$$$$
L^{2} \text { i }
$$

11 quart. fig. 5 . 274 .

Agus bleu a bandes
brunes. Jingr.
Envergure, 21 mill.- - Liles d'un noir brun, semées d'atomes d'un bleu argen- Dans une gr'ande pararcen- tic de la France, dans les le plus petit des Polyom te. Dessous d'un gris de perle, avec une bois sees, les licux pier-mates. Comme dans lunule centrale, une ligne courbe de pe- reux, sur leshautes her- $A$ cis, on aperçoit soutits points ocellés, mais point à la base bes. En juin et aont. vent près du bord marales supérieures; série de points des Chenille verte, aree ginal des traces de lumêmes ailes courbe, mais non sinuée. une ligne dorsale d'un nules.

ㅇ D'un noir-brun uniforme sans ato- rouge brun bordé de Il riest pas rare aux mes bleus. jaunâtre, puis de chaque environs de Châteaum côté une autre pareille dun et de Chartres.

tormée de traits obliques, ct cnfir, une autre latétale jamnatre. Se trourc cn mai et juillet sur l'Astragalus cicer.

Chrysalide jaunatre ponctuce de noir. Linvergure, 52 mill. - Ailes minces, Dans une grande par- Il est commun, et ai-
d'un bleu-violatre pale, avec une bor- tie de l'Europe, dans les me à voltiger autour dure noire très-fine s'elturgissant al scm- jardins et dans les bois. des buissons. Quand il met des supériew'es, qui ont la frantre li- En avril, mai, juillet et est bien frais, les nergirement entrecoupee. Dessous d'un aont. vures sont marquées en blanc bleutre, arec un arc central et Chenille verte, arec blane sur les ailes suune ligne transverse de points noirs pe- une raie dorsale plus périeures, surtout chea tits et non ocelles.
Ioncée, tête et pattes la femelle. Nous avons 
o Avec la bordure trés-large et un|noires. Vit sur le Rham-fiouré sa chenille en are noir aux supérieures, et des points nus frangula et le lier- septembre sur les fleurs marginaux de la même conleur aux infé- re (Hedera helix), dont du lierre. rieures.

clle mange les fleurs.

En juin et septembre.

Chrysalide brunâtre,

ponctuée de noir.

PHELETES.

Ochs. Bdv. God, pl. 25 x. 6ig. 5-6. $548-549$.
Atys. IIub.

Envergure, 28 mill.-Ailes d'un bleu

Suisse, Alpes, Laponic. En juillet. che. Dessous d'un cendré yerdlitre; suune séric de points noirs ocellés (manquant très-sourent); inférieures aree deux rangs de grandes taches blanches on jaunálres currondics.

옴u brun noir uni.

GYLLAIUS.

Envergure, 52 mill. - Ailesd'un bleuFab. Bdr. God. pl, 1 violatre vif, avec une bordure noire un quait. fig. 3.

Damoetes. Ochs. Ilub. $266-368$.

peu large, surtout it l'angle apical. Des-

sous cendré; les supérieures avec une
lunule et une série de gros points noirs

Suite de l'Argus bleu a ocellés; les inféricures avec la base trèsbandes brunes ( $86 \mathbf{~} \mathbf{~}$. o. M.). Engir.
Dans presque toute Ilvarie pourlatailleet l'Europe, dans les prés, la teinte du bleu qui est les bois humides, ctc. quelquefois, mais rareEn mai, juin et juillet. ment, du même ton que Chenille blanche, gri- dans Pherctes. On roit se, jaune ou rose ou de aussi rarement un point largement teinté de vert argente, et une nuances intermédiaires basilaire sous les ailcs série de points acellés pllus petits qu'aux entre ces couleurs, avec supérieures. Il cst comsupéricures et manquant souvent tota- une lignedorsale rouge, mus. lement.

les carènes du dos plus

+ D'un brun noir, ayec le disque pâles que le fond et plus on moins largement saupoudré de marquées aussi d'une libleu riolet.

gne rouge plus ou moins oblique, enfin une ligne: latérale palle; stigmales blancs et tête brune. On la trouve en juin ct juillet sur les Mertirago, les Trifolium, Onolirychis, etc.

Chrysalide d'un gris cendré, étranglée it sa partie supéricurc, bombée it son abdomen, et marquee d'une lime dorsale brune et de deur rangs de points de la même couleur. S'attache it même les plantes basses.

Envergure, 26 mill.-Ailes d'un bleu

Provence, iles d'Hié res. En arril et mai. Bdr. Icon. pl. 3\% fig. violatre, avec une bordure noire assez
\{-6.
large, surtout aux supérieures, et la
frantre grisatre. Dessous d'un cendré Dup. Suppl. pl.8. fig. $\begin{aligned} & \text { frange grisitre. Dessous d'un cendré } \\ & \text { foncé, supéricures arec un are central }\end{aligned}$
- -5 .

SAPORTI.

IIul. $92 x-925$.

très-ctroit et une rangíe courbe de cing points ocellés trés-gros, surtout ceux du
Il n'est pas très-commun. Son rol est rapile, et il aime it se poser les fleurs des pritipieds au-dessus de la mer. Les femelles sont fort rares. bas; inférieures avec la base d'un rert grisâtre, quelques points ocellés trèspetits et une scrie ante-marginale de taches oculaires plus foncées.

o D'un brun noir, arec le disque bleu et une lunule noire sur les supéricures.
Il vole sur les montagrnes sèches et arides et aime à se reposer sur le thym. Même observation quant aux nerrures que pour l'Argiolus. II II'est pas encore trèsrépandu dans les colleclions, quoiqu'il ne semble pas rare dans les pays qu'il habite.

Ouand M. Boisdural le décrivit dans son index et le nomma MIolanops, il figurait dćja depuis long-temps dans Irubner sous le nom de Saporta, et nous le lui aurions restitué si nons 
MaRCHANDH. Bdr. Revue L̈ntom.
D'un blen un peu plus pâle. Dessous des quatre ailes un pen plus foncé, et ou du Mpanne, bois de pins tous les points ont disparu, excepté la virons de Barcelonenlunule discoïdale des quatre ailes et un En mai. point vers le bord interne des supéricures.

LYSIMON. Finvergure, 22 mill. - Ailes d'un vio0clss. God. IIub. 534- Let luisant, arec une large bordure brune 555.

et la frange du même ton. Dessous d'un

Midi de l'Espagne.

|n'avions pas craint qu’on ne le confonde arec un autre P'olyonmate (notre Sebrus), que M. Duponchel a nommé $S a-$ porla.

Nousn'aronspointyu ce Polyommate en nature; d'aprés la firure et la description, il nous semble une simple variété de Melanops.

Bidv. Icon. pl. 17. fig. brun jaunâtre; supéricures avec une z-8. lunule centrale, un point basilaire et Dup. Suppl. pl. 8. fig. une rangée flexueuse de six ì sept au$6-7$.

tres points plus gros, ocellés; inféricures n'ótant pas verdâtres d la hase, et marquées de points ocellés plus petits qu'aux supérieures, avec une lunule centrale et deux rangs anté-marginaux de taches brunes peu apparentes.

o Brune, avec un peu de violet sur le disque des quatre ailes.
Il se trouve aussi au Bengale, d Madagascar, A l'ile Bourbon, en Barbarie et en Egypte, d'où proviennent les individus décrits. Ils y ont été pris ì la fin de févier. la même époque? Il esı rare dans les collections. parait-il en lespagne a

(55) Une bande arquée de points noirs sur les ailes supéricures, au moins dans l'un des deux scares.

EREBUS. Ochs. God. Hub. 260262.

Dup. Suppl. pl. $13 . \mathrm{fig}$. $1-2$.

Bdv. Icon. pl, 2, fig. i-6.

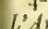
brienes. ble il bandes bruenes. Eingr. 86 A. B. $C$

ILCON. lab, Ochs. Hul, 263 265.

bup. in notis.

Bur. Icon. pl. 13. fig。 $1-5$. Euphemus ('olo pl. a tig. 3 .

fig, 1-2, var. quées.
Envergure, 55 mill. - Ailes brunes, Allemagne, Suisse, Nous avons pris assez saupoudrées de bleuviolatre sur le dis-Alsace, environs de communément cette esque, arec un are discoildal et une ran- Dijon et de Colmar, en- péce dans les prairies gée transverse de points noirs un peu droits montueux. En élcvées et sylvatiques oblongs. Dessous d'un brun bistre, avec juillet et aont. un rang de points noirs légèrement cerclés de gris et une lunule sculement aux supéricures.

et de Schelestadt. En Suisse, au contraire, elle est raxe; e'est dans les cantons de Berne et de Genère qu'il faut la chercher.

peu plus claire.

Envergure, 55 mill.-Ailes d'un bleu

Dans une grande par- Cette espèce, qui se violet, arec une bordure noire assez lar- tic de la France, dans trouve abondamment ge et quelquefois un petit croissant dis-les clairieres des bois. dans certaines localités coïdal, mais sans aucun point noir. Des- Vers la fin de juin. des environs de Paris, sous d'un brun cendré, arec une linule centrale souvent précédée d'un ou deux de points noirs ocellés et un rang antemarginal de lunules légèrement mar-

o Plus grande, d'un biun noir, avec le disque saupoudré de bleu violet et max'gué aux supérieures d'une lunule discoïdale et d'une séric sinućc de points noirs. Dessous plus sombre et mieux marqué. se rapporte bien al l'Euphemus de Godart. Quant it son Alcon, nous pensonsqu'ilu'en estru'une variété; cet Alcon a quelques rapports arec l'indisidu figuré par M. Bdv. (Icon. pl. 13, fig. 7-8) et qu'il regarde avec doule comme variété d'Luplémus.

Tous deux viennent des environs de Lyon, et i] serait possible que tous deux fussent des variétés locales d'Alcon, ee que nous ne pourons décider sur les figures seules. In tous cas I'dlcon de Godart n'est point notre Euphénus. 
H.UPHinus. | Envergure, 55 mill-Ailes d'un bleu| Allemagne, Russic,| Nous l'avons pris aux|

Ochs. Hub. $25 ;-259$.

Dup. in notis. - Balr. Icon. pl. 15. fig.

violet, plus pâte et comme argentévers la Suisse, est de la France. environs de Neuf-Bricôte el vers la bordure, qui est large, dans les prairies humi- sach. Il n'est pas très-

mirquéc et une série un peu arquée vers

la cûte de points noirs. Dessous d'un brun assez foncé, avec les mêmes taches que chez Alcon.

ㅇ Plus grande, d'un bleu violet, avec une très-large bordure brune, une série de gros points noirs un peu arqué sur les supérieures, et une autre série plus courte sur les inféricures. Dessous plus sombre et micux marqué.

Nlitos.

lin. Fab. Ochs, Hub. $254-25 t i$.

biv. fiod. pl. 11. fig. 2 . ('t pl. 11 quart. fig. 1. Subte de l'Argus bleu a bandes brunes, Jingr.

$$
\begin{aligned}
& \begin{array}{l}
\text { pericures qu'aux inferieures. Dessous } \\
\text { d'un cendré un peu jaunatre, avec la }
\end{array} \\
& \text { base des infericures verdatre et ane mul- } \\
& \text { litude de gros points noirs tris-saillants, } \\
& \text { dont la série intermédiaire et la lunule } \\
& \text { discoïdale fortement cerclées de jaunî- } \\
& \text { tre, surtout anx inférieures; supéricu- } \\
& \text { res ayant ordinairement deux points } \\
& \text { ocellés à la base. } \\
& \text { o plus grande. it bordure plus large } \\
& \text { et it points beaucoup plus gros; taches } \\
& \text { marginales ocellies du dessus moins } \\
& \text { insibles. }
\end{aligned}
$$

répandu dans les collecde la confusion qui a été jetie dans son histoire. Sa femelle se distinguera de celle d'Atcon par la bordure mienx arrêtéc, les points des secondes ailes et la rangéc des premières. qui est bien plus droite vers le bord interne. Quant au mâle, les points du dessus le distinguent suffisamment.

On rencontre parfois cles variétés femelles oi le noir a absorbé presque toute la surface supérieure des ailes. On le trouve cà et là aux environs de Paris, mais jamais abondamment.

Cette espece et les deux précédentes, ont la frange un peu entrecoupéc.

\title{
(56) Division II. IIEODES, Dalm. POLYOMMATUS, Bdr.
}

\begin{abstract}
Chenilles pubescenles, convexes, soutent un peu allongées. - Chrysulides courtes ot diprimés antérieurement. - Ailes infiricures ayant l'on

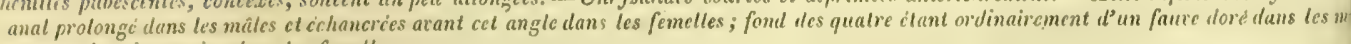
et semé de points noirs tans les fomclles.
\end{abstract}

\section{(Ises Bronsés. Lat. God. Famille 8. Rutili. Ochs.)}

IILHL. $\quad$ Envergure, 28 mill.-Ailes supérieu-| Allemagne, environs II est commun dans Fab. Uchs. Bilv. Iluh. res d'un faure orangi entierement recou- de Leipsick, Suisse, le nord de l'Illemagnc, 551-555. $\quad$ rert de violet, arec une bordure et des dlans les prairies humi- on il parait en mai et God. pl. 25 w. fig. 5-6. points noirs; inférieures brunes, recou-des des montagnes. En aont. Lu Suisse, au conL'Arges myop riolet. vertes de violet sur le disque, avec des mai, juin, juillet et traire, ou il est rare, I.ngr, points noirs et une bordure orangée. aont.

c'est en juin et juillet Dessous des supérieures orangé, avec Chenille d'un vert qu'il faut le chercher. des points ocellćs et une série anté-mar-pâle, ayant sur le dos ginale de points noirs surmontés de che- une ligne plus foncée. vrons blancs; dessons des inférieures Tête ct anus jaunes ou l'un brun faure, avec les mêmes carac- rougentres. Vit sur la lieres, et ayant de plus une bande anté-patience (Rumex Patienmarginale d'un rouge ponceau. tia). Se trouve en juin of $A$ ailes supericures plus arrondies et septembre. "it w'arsant de violet que quelgues points. Chrysalide d'un brun clair, pirtuéc de noir et ayant quelques espaces latéraux blanchitres. 


\section{G. POLYOMMATUS.}

Fab. Bdr.

XANTHE: od. pl. $9 \mathrm{sec}$. fir. 5. et terminale de lunules fauves. Dessous tie de l'Europe, prés et mais plus rare dans 10 sec. fig. 1. d'un jaune pâle et un peu verdâtre, arec clairiéres des bois. En prés. On distioguera sa Circe. Ochs. Hub. 334- beaucoup de points noirs légérement mai, juillet et aoat. phenille decelles d'Ado336.

liArgus myope. Engr. ocellés, dont quatre groupés au centre Chenille d'un vert nis et de Corydon, auxdes inférieurcs et une bande anté-mar-pomme, avec le, vais- quelles elle ressemble ginale fauve renfermée entre deux sí- seau dorsal un peu en-beaucoup, en ce que la ries de points noirs.

foncé et bordé de chaque bordure latérale jaune

o $\Lambda$ ailes supérieures plus arron- côté par des crêtes n'est pas réunie a l'adies, fauves, marquées de points noirs triangulaires d'un vert nus.

et ayant en dessous le disque des mêmes pále, d'un jaune verdâ-

TUERSAMON Fab. Ochs. Bdv. God. pl. 21 v. fig. $7-8$. Xanihe. IIul. $346-348$.

GORDIUS )chs. Bdy. Hub. 545345. liod. pl, $25 \mathrm{w}$. fig. 1-2. Engr, ailes faure.

tre ou d'un jaune pur.

Côtés saillants, bordés

d'une ligne jaune ou

d'un vert pále qui va

du troisième anneau it

l'anus, mais sans s'unir

avec relle du coté opposé.

Stigmates roussátres

tête et pattes écailleuses

de la même couleur. Se

trouve en juin et sep-

tembre sur le genêt.

(Genista scoparia.)

Chrysalide brune hé-

rissće de petits poils,

attachée aux tiges des

plantes.

Envergure, 35 mill.- - 1 iles d'un pon- Italie, IIongrie, Rus- - Cebeau Polyommate ceau vif et foncé, glacées de violet sur sic, $\Lambda$ utriche. En juillet. n'est pas très-commun. leurs bords. Supérieures avec une bordure noire, le plus sonvent sans aucun $\quad$ le midi different sensipoint, quelquefois avec un ou deux points noirs et toujours avec la transparence de ccux du dessous. Inférieures plus sombres jusqu'aux trois quarts, avec une bordure noire surmontée d'un rang de points de même couleur. Dessous des supérieures d'un fauve plus foncé au bord terminal, avec des points noirs trés-ocelles. Dessous des inféricures gris, arec des points semblables, ct une série anté-terminale de taches faures entre des points noirs.

o Plus arrondie, d'un beau fauve orangé, arec une multitude de points noirs dont devix au bord interne des supéricures allongés et parallèles.

blement de ceux de Hongrie. Dans ces derniers, les mâles sont d'un rouge plus sombre et ont les ailes inférieures très-entières. Ils se rapprochent beaucoup du Chryseis. Les premiers au contraire sont plus clairs, tant en dessus qu'en dessous, plus arrondis, et les inféricures sont un peu dentées et pourrues non loin de l'angle anal d'un petit prolongement presque en queue; mais toutes les femelles sont à peu près identiques et se rapprochent de celles de Gordius.

Envergure, 58 mill. - Ailes d'un Alpes, Pyrénées, Il est très commun. faure orangé vif, glacées de violet France méridionale, Nous l'avons pris en avec de très-gros point noirs, dont les Lozère, etc., etc., dans abondance dans les endiscoïdaux sans reflet violet. Dessous les montagnes. En juin droits secs et arides des. des supérieures d'un fauve jaunatre et juillet. avec des points noirs d peine ocellés. Dessous des inférieures d'un cendré jaunfitre, avec beaucoup de points ocellés et une bande fauve continue renfermée entre deux rangs de points noirs.

f D'un fauve plus pale sans reflet et arec les points noirs plus gros. environs d'Ax et de Ulontpellier. Il aime d se reposer au soleil, sur les rochers. Les femclles sont plus faciles at prendre que les males. 
HIEnE. Envergure, 56 mill. - Ailes d'un|. Alpes, Alsace, Lor-| Cette espèce est très-|

Fab. Bdy. God. pl. 25 faure ponceau presqu'cntiercment cachid raine, IIongrie, etc., commune dans la forêt w. $\mathrm{fg} \cdot 3-4$.

Hipponoe. Ochs. sous un reflet violet très-rif, légère- dans les bois. En juin de Wolckamentre Neuf-

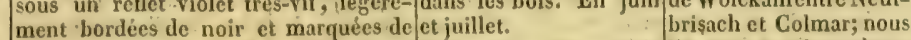
Lampetic. II bb. 356- quelques points de cette couleur. Des- Chenille trés-moni- I'y avons prise abon559. sous cendré, a base bleutitre, avec beau-liforme, d'un vert mat, damment sur les fleurs

$L^{\prime}$ Argus satiné. $9^{5}$ C. D. coup de points' ocellés assez petits et aveo des enfoncemens de l'Eupatoire.

c. bis. Engr. une série anté-terminalẹ de petites ta- sur le dos et sur les ches faures comprisés chacune cntre côtés, et des poils courts deux points noirs.

o Plus grande; d'un brun noir, avec vioirâtres, tête brunâtre. le disque d'un fauye obscur marqué de Vit en arril et mai sur points noirs, dont une série discoîdale l'oseille samvage ( $R u$ mal aligné ; inférieures ayant une-bande mex acetosa).

anté-terminale d'un fauve orangé, Chrysalide déprimée étroite, marquée de points noirs et sou- sur le dos et a abdomen vent surmontée de points bleus. ... très-renflé, grise, arec des points bruns plus des points brun
serrés sur le dos.

CIIRYSEIS. Envergure, 52 mille- - Ailes d'un . Alpes de la Suisse et Nous avons pris cette

Fab. Ochs. Bdr. Hub. auve ponceau vif, avec une bordure de la France, départe-belle espéce dans les 337-358.355. assez large; la côte des supéricurés et ment de la Somme, en- clairières humides de la God. pl. 9 sec. fig. 4. une partic des inférieures d'un noir virons de Paris, dans forêt d'Ilallate, près de et 10 sec. fig. 2. glacé de violet foncé, et un seut trait les bois humides, les Pont-Sainte-Maxence. L'Argus satiné chan-discoidal formé de deux petits points noirs. marais et les prairies Malgré nos recherches geant. $9^{5}$ A. B. E. . . . Dessous d'un cendré jaunâtre foncé, montagneuses. Én juin sur l'indication de Gobis. Engr.

bande anté-marginale, souvent très-

courte aux inférieures, faures et beaucoup de points ocellés.

o Toute brune, aveo le disque des supéricures légèrement fauve, et marqué de deux points discoildaux et de deux séries de points anté-terminaux bien alignes; inferieures arec une bordure anté-terminale d'un fauve pur.

EUnYice.

Hub. $339-342$.

God. pl. 22 v. fig. 5-6.

Il diffère du Chryseis, en ce que la bordure est plus étroite et nullement juillet.

Hautes Alpes. En dart, nous ne l'avons jamais trouvée plus près de P'aris. Sa femelle se distinguera de la précédente par sa double série bien alignée de points noirs et par sa teinte bien plus foncée en dessous.

Eurybia, Ochs.

Chryseis. var.? Bdv.

glacée de violet, en ce que le point discoidal des premières ailes est à peine sensible, et même souvent tout-ĥ-fait nul; en ce que le dessous est d'une teinte un peu plus cendrée, sans aucun restige de couleur fauve, ni aux supéricures, ni aux inféricures.

o N'ayant point le disque fauve en dessus, et ayant ì peine quelqques taches de cette couleur' ì l'angle anal des secondes ailes en dessus et en dessous.

HIPPOTHOE.

Envergure, $5 \rho$ mill.-Ailes d'un

Ouest et Est de la Nous l'avons pris à Lin. Fab. Bdv. Ochs. rouge ponceau très-brillant, arec une France, Ilongrie, ete., la fin de mai dans les Hub. $352-554$.

God. pl. 9 sec. fig. 5 . et ro sec. fig. 3.

bordure éroite et un arc central (ac- dans les lieux marécaCossés des fortifications compagné quelquefois d'un point) geux. Dansles premiers de Neufbrisach, et en noirs. Dessous des supérieures d'un jours de juin et d'aoot. nout dans les prairies $L$ 'Argus satiné daches fauve pâle, plus foncé vers les bords, noires. Engr.

aveo beaucoup de points ocellés et une lunule centrale noirs. Dessous des inférieures d'un 'endré clair, avec la base largement bleuâtre et unc bande anté-marginale d'un fauve rouge, continue et renfermée entre deux rangs de petits points noirs.

\& Ayant plusieurs points noirs sur les supérieures, et les inférieures d'un brun noir avec les nervures et une marécageuses du bord du Rhin, ou il est commun. 
bande terminale fauve, marquéc inférieurement de gros points noirs.

Dispar. Ne differc d'Hippothoe que par sa 13. fig. 5-6. couleur plus vive.

Bdv, Icon. pl. ro: fig.

1-3.

Ilippothoe. var. Hub. 966-968.

VIRGAUREIE:

VIRGAURE.E.
Envergure, 53 mill.- - Alles un peu Alpes, Pyrénées, de Cambridge et de mîles qui avaient jusll untington. En juin et qu'd 45 mill d'enrerjuillet. gure. M. Lefehrre pense que cette espece est le véritable Ilippothoe, dont nous n'aurions en France qu'une variété plus petite. II ub. $349^{-351}$. le mâle), d'un faure ponceau trìs-bril-juillet et aoot.

Il est commun; nous God. pl. 9 sec. fig. 6. et lant, arec une bordure noire assez large ment dans les bois de 10 sec. fig. 4 . L'Argus satiné. Engr. et sans taches sur te disque - a d'un vert sapins des environs laure jaches sur le disque. Dessous d'un foncé, avec une bande d'Ax et dans les Pyrépoints points etune ligne de taches blanches aux par un filet veit et deux sur les fleurs qui croisinféricures.

o D'un fauve moins vif, avec unc lignes laterales d'un sent au bord des ruismultitude de taches brunes, et les in- tesécailleusesnoires. Sc surtout la femelle; celférieures presque entièrement envahies trouve en juin et sep- les qu'on prend dans le par cette couleur.

tembre sur la verge Jura ont une teinte géd'or (Solidago Virga au-néralement plus foncée rea) et la patience ( $R u$ - que celles des Alpes. mex patientia).

Chrysalide d'un brun jaunatre, avec l'enreloppe des ailes plus foncée. \begin{tabular}{c|c} 
OTTOMANUS. & $\begin{array}{c}\text { Enrergure, } 50 \text { mill. - Ailes d'un } \\
\text { Lefebre. Dup. Suppl. }\end{array}$ rouge ponceau brillant, avec une bor- rarin, Thérapia. Ea-
\end{tabular}

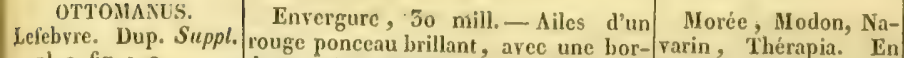
pl. 9 . fig,$x-2$. Bdv. Icon. pl. 10, fig. dure noire. Supérieures entières, avec mars et juin. $\{-5$.

de lunule discoidale, suixie ordinairemen de trois a quatre points noirs; inféricures échancrées avant l'angle anal (même dans le maile), arec la bordure noire dentée. Dessous des supéricures d'un fauve clair, avec des points noirs légèrement ocellés; dessous des inférieures d'un cendré jaunatre, plus obscur au bord marginal, arec des points semblables et une bande anté-terminale, maculaire, $d^{\prime}$ un rouge ponceau, dont la tache anale et quelquefois la précédente plus grande et lunulée.

ㅇ Inconnuc.

IUH TIAS

Lin. Fab. Ochs. Bdr Hub. 562-363. Yar. accid, $756-737$ God. pl. 10. fig. 1. Le Bronzé. Engr.
Envergure, 28 mill. - Ailes brunes;

\begin{tabular}{l|l|l|} 
& Volontiers sur les céréa- \\
les.
\end{tabular} fauve deré an bois, prés, champs de partout. It n'est pas rafauve doré semé de points noirs; les luzerne, etc. En avril, re de trourer des indiinférieures échancrées arant l'angle aoat et septembre." ? anal (même dans le mâle) et ayan (Chenille d'un rert bande faure des inféune bande anté-terminale du même pâle, avec une lighe rieures est surmontêe fauve, appuyéc sur despoints noirs ter- jaune ou rougeâtré lè d'un rang de point bleus. minaux. Dessous des supéricures fauve tong du dos, et une' li- La chenille est mal consur le disque, avec des points noirs as- gne latérale semblable. nue. Ilub. fig., $n^{\circ}-\mathbf{5 6 -}$ sez gros légèrement ocellés; dessous Vit sur l'oseille sauvage 757 , une variété où le des inférieures $d^{\prime} u n$ cendré brun, avec (Rumex acelosa'). de petits points noirâtres peu marqués, $\begin{gathered}\text { Chrysalide d'un brun dublanc; nousnél'avoñ } \\ \text { dún }\end{gathered}$ et une ligne anté-terminale maculaire, clair, ponctuée de noir. jamais vie en hature. rougeâtre, composée d'arcs dont l'anal plus grand.

q Semblable.

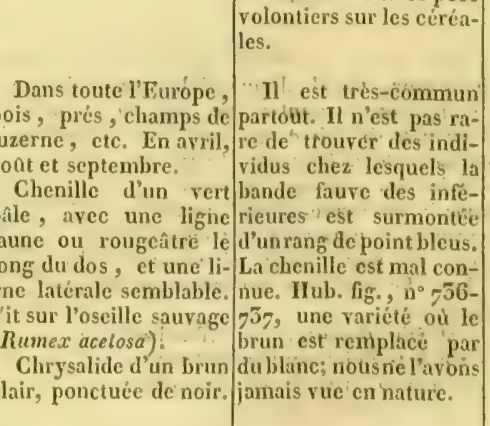

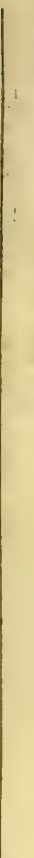

dans les collections. Sur quatre exemplaires mâles que - nous arons vus, un seulmanque de la serie de points noirs
du dessus des supérieures. It a en dessous quelques rapports avec le Phlaas. Les figures de MM. Bdv, et Dup. sont un peu trop petitaille du Vigaurea; son vol eșt vif et il se pose 
I Melanopaleas. Lefeb. Collect. Nobis. ailes supéricures sont entierement brunes environs de Paris. et seulement légèrement saupoudrées de faure doré à la base; les ailes inférieures sont aussi qquelquefois dépourvuesen tout ou en partie de la bande antéterminale fauve.

BALLUS.

Fab. Ochs. God, Ilub. $360-361.550$.

Dup. Suppl. pl. 7. fig. $1-3$.

Bdv. Icon. pl, 10. fig. $6 \rightarrow 7$.
Envergure , 28 mill. - Liles presque Portugal, Espagne, Il a été long-temps rondes, d'un brun cendré un peu plus Sicile, Provence, Pyré-très-rare dans les colclair sur le disque, arec quelques petits nées, environs de Per- lections, mais il y est points fauves a l'angle aual des infé- pignan et d'Hières. En $\begin{aligned} & \text { répandu maintenant. Il } \\ & \text { a beaucoup de rapport }\end{aligned}$ rieures. Corselet garni de poils verts. mars. - Dessous des supériéures ayant le disque chente avec une lí-sieurs auteurs l'ont plafe blanc; dessous des inférieures cou- gne dorsale maculaire cé cntre lui et Rubi, de blanc; dessous des inférieures cou- gne dorsale maculaire ce cntre lui et Rabeu et marqué de quelques pelits points brun-rouge et coupée tention, on s'apercevra rouges accolés di des points blanes. dans le milieu par une qu'il n'a d'autreressem. o $\Lambda$ yant le disque des premières ai- ligne bleue; puis une blance arec ce dernier les, et une large bande anté-terminale série de petits traits que la couleur verte du aux secondes, d'un faure clair sans éclat. obliques, d'un rouge dessous des inférieures; violatre, puis une ligne encore y a-t-il une latérale de même cou-grande différence dans leur. Tête brune, pre-- ce caractère commun. mier et dernier anneau (Le Rubiappartient d'aillavés de rougeâtre. Se leurs évidemment par troure en mai sur le $L o-$ sa chenille et les lignes tus hispidus.

Chrysalide d'un brun division suivante.) Sa marron.

(57) Division III. AUROTIS. Dalm. (Lycana et Thecla. Bdv.)

Chenilles pubescentes, moins ramassées que celles de la dirision précédenle, et ayant quelquefois la parlic antéricure tris-aplalie.-Chrysalides courtes, convexes en dessus, légèrement aplaties en dessous. - Ailes inferieures ayant prés de l'angle unal un prolongement souvent tris-long et en forme de queuc.

(58) $\{$ Ailes inféricures dentées, mais dépourvues de queue.

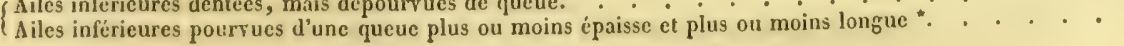

\section{(59) Ailes inférieures dentées, mais dépouroues de queue.}

EVIPPUS, Bdr. Hub. 366-36\% 。 God. pl. 22 v. fig. $1-2$. Roboris, Ochs. Envergure, 54 mill. - - Ailes d'un
brun noir, avec le disque violet; inférieures ayant en outre une sirie anti-dans les montagnes. Ln terminale de trois a quatre points de la juin et juillet. même couleur. Dessous d'un gris jaunâtre satiné, arec une ligne anté-terminale interrompue, d'un bleu métallique, surmontée d'un rang de taches fauves, puis de points noirs chevronnés de blanc. Le tout plus prononcé aux inférieures.

\% Plus grande, ayant ordinairement six points violets au lieu de trois sur les inférieures, et le disque des supérieures moins largement violet.
Nous avons pris ficiquemment cette espece a Castclnau, pris Monpellier. Elle a les mêmes habitudes que le Poly. Quercuss, et aime d se reposer sur les feuilles du chêne vert et du prunier sauvage.

\footnotetext{
- Voyez la note au bas du Polyom. Rubi.
} 


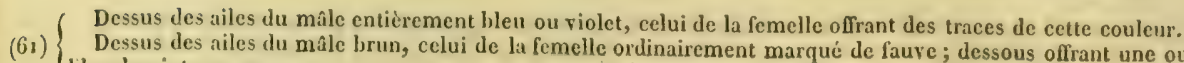
(blanches interrompues. . . . .

(62) Dessus des ailes du male entiirement bleu ou violet, celui de la femelle offrant des traces de celle couleur.

Genre LYCENA. Bdv.

(63) \{ueue médiocrement longue et plus épaisse à son insertion; inféricures un peu denties. . . . . . . (64) Queue longıe, très-grêle et entièrement filiforme; inférieures très-cntières. . . . . . . . . . . . . . 65 (65)

(64) Quette médiocrement longue et plus ipaisse d son inserlion; inferieures un peu dentees.

QUEncus. | Envergure, 54 mill. - Ailes d'un| Dans toute l'Europe, Il est commun, mais Lin. Fab. Ochs. Bdv. brun noir glacces de violet trés-fonce et un dans les bois, en juin et assez difficile à prendre, Hub. 368-37o. peu changeant, dessous d'un gris satiné, juillet. parce qu'il voltige an Var. $q$ accid. 621 (trois avee une ligne blanche continue, légè̀- Chenille d'un rert sa- sommet des chênes. On points faures sur les rement ondec, et deux taches roussâtres le ou roussûtre, arec le s'en saisit plus facilesupéricures). it l'angle interne; celles des supérieures vaisseau dorsal enfoncé mentl'aprés-midi quand poly. du chêne. God. pl. irrégulières et ombrées de noir, celles et découpant chaque il descend sur les taillis;

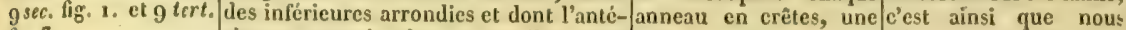
fig. 5 .

\begin{tabular}{|c|c|c|}
\hline $\begin{array}{l}\text { rieure marquée dans son milieu d'un } \\
\text { point noir non métallique. } \\
\text { \& D'un brun noir, avec une large ta- } \\
\text { che bifurquie d'un violet brillant sur les } \\
\text { supérieures. }\end{array}$ & $\begin{array}{l}\text { suite latérale de lignes } \\
\text { obliques jaunes, et sur } \\
\text { le dernier anneau, qui } \\
\text { est plus long et en fer à } \\
\text { cheval, un dessin longi- } \\
\text { tudinal et bizarre. Tête } \\
\text { brune. Vit en juin sur le } \\
\text { chêne. } \\
\text { Chrysalide rousse on- } \\
\text { dée de taches plus clai- } \\
\text { res. }\end{array}$ & $\begin{array}{l}\text { l'avons recueilli en } \\
\text { abondance au bois de } \\
\text { Boulogne. Nous avons } \\
\text { vu en nature, dans la } \\
\text { collection de M. Mar- } \\
\text { chand, la jolie variété } \\
\text { figurée par Hubner } \\
\text { sous le no } 621 \text {, et Dal- } \\
\text { man en parle de son } \\
\text { cutte. Mais celle qu'il } \\
\text { cite et celle que nous } \\
\text { avons vue n'avaient } \\
\text { qu'un scul point fauve. }\end{array}$ \\
\hline
\end{tabular}

(65) Queue longue, tris-grèle et entierement filiforme; inférieures trés-entières.

BOETICUS. | Envergure, 34 mill. - Ailes d'un| France centrale et Il est commun à Lin. Fab. Ochs. Bdr. violet assez foncé, arec une bordure méridionale, etc, dans Montpellier et à La RoHub. $373-375$ (la \& brune et deux gros points noirs a l'an-les parcs, les jardins. chelle; mais dans nos est une var. accid.). gle anal des inférieures. Dessous d'un En aoot et septembre. environs on ne le trouGod. pl. 9 tert. fig. 4. cendré jaunâtre, arec des lignes blan- Chenille d'un vert ve que de loin en loin. et $10.6 \mathrm{gig} .2$. ches ne dépassant pas la cellule discoidale olivâtre, avec le dos M. Marchand l'a élevé Le Porte-Queue bleu sur le disque des supérieures; inféricures jaspé de rouge. Vit en à Chartres, en quantité strie. Engr. ayant d l'angle anal deux points noirs juin et en juillet dans les en 1827 , et nous-mêmes cerclés inféricurement de vert métalli- siliques du haruenaudier avons trouvé cette anque, surmontés de faure et dont l'ante- (Colutea arborescens) née (1834) deux baguerieur beaucoup plus gros. o Plus grande, brune, avec le disque légumineuses.

naudiers dont les graiviolet. Chrysalide jaunatre, tes dévoríes par sa cheponctuée de noir. nille.

Le $n^{\circ} 375$ d'Hub. représente une femelle qui a sur les ailes supérieures deux séries, l'une marginale et ocellée, l'autre anté-marginale et simple de taclies jaunâtres. 
TELICANUS. | Euvergure, 27 mill. - Ailes arron- France méridionale, Nous avons pris cette

Ochs. Bit.

Hub. 3-1-572, 555- points noirâtres près de l'angle anal. parcs, les jardins. En. les espèce a Montpellier 554 . Dessous d'un cendré brunâtre, traversé juillet et aont.

sur les fleurs des légu-

God. pl. $22 \mathrm{v}$. fig. 3-4. de nombreuses lignes blanches très- Chenille veloutéc, en graude quantité.

fexueuses, toutes celles des supéricures d'un rouge purpurin, atteignant le bord interne et les anté- avee des lignes fines et marginales formant une série de taches obliques sur tout le oculées; inférieures ayant à l'angle anal corps et une raic dorsadeux taches oculées d'un vert métalli- le plus foncée. 'Vit en que bordées de roux el égales en gros-aont et septembre sur seur. la salicaire (Lythrum o D'un brun noir, arec le disque salicaria), dont elle previolet et quelques points plus foncés. ' fére les fleurs.

Envergure, 30 mill. - Ailes d'un violetclair, supérieures avec un trait discoidal noir, inférieures avec deux points de cette couleur a l'angle anal. Dessous d'un cendré jaunâtre, les supérieures avec trois bandes plus foncées, un peu maculaires et bordées de blanchatre, la première au bout de la cellule et réIDION. duite à deux points, les deux suivantes IIul). $820-821$. atteignant le bord interne; inféricures arec quatre points ocellés à la base et quatre bandes pareilles à celles des supérieures, les deux discoïdales irrégulières et sinuíes, les deux anté-terminales paralleles et dont l'inféricure terminée à l'angle anal par deux points noirs cerclés supérieurement de fauye.

오 Inconnue.

Envergure, 29 mill. - - Ailes d'un bleu violet vif, avec la bordure noire et tie del'Europe. Endroits bien frais la partic in-

Dans les individus quelques points terminaux de cette cou- herbus, prés et clairiè-férieure de la tache fauleur aux inféricures. Dessous d'un gris res des bois. En mai, ve anale est souvent

de perle, avec un trait discoidal allongé, juillet et aoat. $\quad \begin{aligned} & \text { saupoudrée de vert mé- } \\ & \text { tallique, comme dans }\end{aligned}$ un bande de petits points ocellés, et une double séric anté-marginale de taches brunes peu marquées; inférieures ayant près de l'angle anal deux petites taches lauves appuyées sur un point et sur-

AMYNTAS

Falz, Ochs. Bdv. Huls, 522-324. God. pl. 9 sec. fig. $2 . \mathrm{et}$ 9 tert. fig. 5. Le petit Porle-Queue. Engr.

TIRL-IAS.

Hub. $319-521$.

Polysperchon. Ochs. . montées d'un petit are noir.

o Brune, arec les deux points faures de l'angle anal apparents en dessus.

Ordinairement plus petit, et dépourve des points fiures de l'angle anal tant en lie piement, midh dc dessus qu'en dessous. Femelle saupou- ha France, etc., etc. lréc de bleuatre en dessus.
Nous ne connaisson cette espèce remarquable que par la figure d'Hubner. Elle paraît tenir à la fois du Boeticus et de l'Amyntas, dont elle n'est peut-être qu'un hybride; mais clle a des caractères si tranchés que nous avons cru devoir la conserver comme espèce. Bocticus, etc., quoique d'une manière moins sensible. On roit aussi très-sourent sur les supérieures un trait discoïdal noir. Le Myrmidon d'Engr. n'est qu'un Amyntas plus petit. Nous l'avons recu de Suisse.

On insiste beaucoup pour séparer ces deux Polyommates, et M. Treitschke lui-même, tout en rapportant dans son dixieme volume un article concluant en fareur de leur réunion, n'en persiste pas moins diles séparer; pour nous, nous prenons fort sourent des Amynlas femelles plus petites et qui manquent des points fauves en dessus; elles sont même plus communes ici que les fe- 


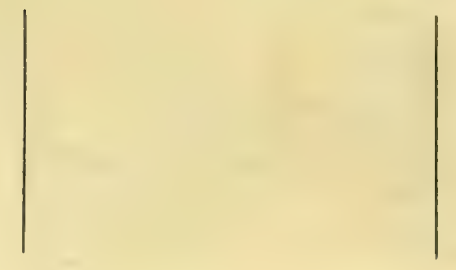

melles d'Amyntas proprement dites, bien que les máles n'offrent : aucune différence sensible. Les antennes sont aussi, comme le rapporle très-bien M. Treitschke dans cette note, très-semblables dans les deux espéces. Dessus des ailes du mále brun, celui de la femelle ordinairement marqué de faute; ilessous offrant une ou deux lignes blanches
interrompues.

Genre TIIECLA. Bdr. .

$(6)$

$\left\{\begin{array}{l}\text { Queue courte, ipaisse, situće à l'angle anal; inférieures fortement dentées en approchant de cet angle *. } \\ \text { Queue longue, filiforme, située près de l'angle anal; inférieures légèrement dentées. }\end{array}\right.$

(68) Queue courte, ipaisse, située d l'angle anal; inférieures forlement dentées en approchant de cet angle.

RUBI.

Lin. Fab. Ochs. Bdv. Ilub, 36, -565.786 .

L'Argus vert, Engr.
Envergure, 28 mill.-Ailes d'un brun| Dans presque toute| Il n'est pas rare. Il un peu luisant. Supéricures ayant ì la l'Europe, dans les bois, aime à se reposer sur côte un point ovale d'un brun terne. sur les buissons. En les feuilles el serait très. Dessous d'un beau vert avec une ligne mars, avril et mai. ' difficile a trourer si on de traits blancs.

o Dépourvue de points bruns i Chenille verte, arec ne le voyait voler, it ne ligne dorsale, un cause de sa couleur, qui rang de taches latérales se confond avee la verobliques et une autre dure. C'est un des léligne au-dessus des pat- pidoptères les plus prétes, d'un jaune clair. coces de nos pays.

Vit en juillet et aoat sur plusieurs espéces de ronce, de cytises, le genêt à balais, ctc

Chrysalide un peu velue, d'un brun foncé, avec les stigmates plus clail's.

(69) Queue longue, filiforme, situe prìs do l'angle anal; inférieures ligerement denteies.

ACACIE Fib. Ochs. Bdr. Hub. $-43-746$.

Poly. de ''Acacia. God. pl. 21 v. fig. 6-7.
Envergure, 27 mill. - Ailes assez larges, d'un brun noiratre, les inféricures
un peu arrondies et ayant a l'angle anal deux ou trois taches faures lunulées. En juin.

Dessous d'un brun cendré clair, à base légèrement verdatre, avec une ligne blanche interrompue, celle des supérieures descendant jusqu'au bord inter$n e$, les inféricures ayant en outre press de l'angle anal une série de taches lau-
France centrale et| Cette espèce, qu'on méridionale, Autriche, a cru long-temps mérietc., etc., dans les bois. dionale, est assez commune aux environs de Chateaudun; mais il est rave de la prendre iraiche.

'Certe queue existe aussi dans la division suivante, mais moins prononcúe que dans Rubi. Dans ce Polyommate la véritable quette est nulle a projremenl parler, et réduite à une simple dent; mais il est si voisin d'Acaciae, et il appartient si évideminent à cette. section, que nous arons mieux aimé forcer le caractère divisionaaire que de l'en séparer, Nous ajoutons cette note afin que l'élève ne puisse s'y tromper. 
|res surmontées d'ares noirs très-légers| et dont l'intermédiaire appuyée sur un point noir. noirs.

o Syant ù l'anus un bourrelet de poils

SPINI.

Envergure, 32 mill. - diles d'un brun Fab, Ochs. Bdr. nofratre; supérieures avec une tache Suisse, midi et est de Nous l'avons pris pluPolvom . Prunellier. costale d'un brun mat; inféricures avec la France, dans les bois sieurs fois dans les bois Gom deux ou trois taches arrondies, fauves, at montueux. En juin et montucux du grand-

$\begin{aligned} & \text { God. pl. } 21 \text { v. fig. 8 }-9 . \text { deux ou trois taches arrondies, faures, al montueur } \\ & \text { Lynceus. Hub. } 69^{2}-69^{5} \text {. l'angle anal. Dessous d'un brun cendré juillet. }\end{aligned}$

duché de Bade, sur les clair, arec une ligne iransverse blanche Chenille d'un vert fleurs de ronce, et aux Lien marquée et légèrement interrom- pomme, avee deux li- ensirons de Montpellier Le Porte-Queue irun ai pue; les inférieures ayant en outre un gnes latérales d'un vert sur le chêne vert. taches bleues, et le rang marginal de taches faures et une plus jaunatre, puis un Ise Lynceus d'Hlub. et eris tache anale grande, carree et entierement rang de petits traits son Spini diffèrent it

Porle-Queue gris tacke anale grande, carré et enticrement rang de petits traits
brun. Engr.
saupoudré de bleu. brum. Engr. saupoutre de bleu. o Manquant de la tache costale aux ombrés de vert foncé, tamment par le dessous, supérieures etayant le disque des mêmes et un sillon dorsal en-bien plus foncé dans le ailes légèrement faure.

foncé et formant des premier; mais sur des petites crêtes dont le figures on ne peut tenir sommet est rosé. Se compte de différences troure en juin sur l'au- aussi légères. bépine et le prunellier. Chrysalide d'un brun clair, parsemée de points noirs dont un oblong sur la tête; attachée à une feuille ou i) une branche.

LYNCEUS. Envergure, 55 mill.-Ailes d'un brun

Dans toute l'Europe,

Il est fort commun. LYNCEUS. Fab. Bdv. God. pl. 9 tert. fig. 1.

i) l'angle anal. Dessous d'un brun un sons, sur les ronces, etc. des mâles qui ont Ilicis. Ochs. Hub. $378-$ peu plus clair que le dessus, avec une En juin et juillet. comme la femelle une 379. limne transverse, blanche, très-interChenille d'un veri tache faure sur les suLe Porte-Queue brun d rompue, a peine sensible aux supérieures pâle, tachetéc de jaune périeures.

taches faurcs. Engr. et n'atteignant que les deux tiers de l'ai-

sur le dos et sur les cô- Nous arons pris cette le; courbe et sinuée aux inférieures, ou tés; tête et pattes écail- année en quantité,volant es en crochet et leuses noires. Vit en arec des Lynceus el des rentre en dedans; inférieures ayant un mai sur le chêne. Acacia, un Polyommate rang marginal de taches d'un faure asChrysalide d'un brun que nous eroyons hysez clair, un peu isolées et de moyenne grandeur.

cair ou jaunâtre, avec bride de ces deux espèAyant sur les niles supéricures une obscurs. tache fauve plus ou moins grande.

Cerar. Hul). 863-866. Ochs. God. Bdv.

Esculi, II ub. 559-560 Var. accid. 690-691.

Poly. du Marronnier. God.
Ne differe du Lynceus qu'en ce que le $\sigma^{7}$ a sur les ailes supérieures une tache faure, et que la $q$ a cette tache plus grande que les Lynceus $q$ ordinaires.

Il est plus petit que Lynceus, dont il se distingue en outre: $1^{\circ}$ en ce que les taches fauves du dessous des deuxièmes ailes sont plus vires, plus petites et par conséquent plus isolées; $2^{\circ}$ en ce que l'avant-dernier trait de la ligne blanche des mêmes ailes est à peine arqué, au lieu d'être en crochet, et mieux aligné arec les autres.
Midi de la France. est tout-d-fait intermé-
diaire, quoique se rap-
prochant davantage du
premier; c'est pourquoi
nous n'avons pas cru
devoir le mentionner
séparíment.

Nous arons vu en nature cette prétendue espèce, qui ne diflere point de notre variété à t.ıches faures du Lynceus.

Nous l'arons souyent pris à Castelnau, près Montpellier, volant sur le chene vert. Il se pourrait que ce Polyommate dat former une espèce, et c'est même i) la sollicitation de l'un de nous que Godart le 
W. ALBBtiM.
Envergure, 53 mill. - Siles un peu Environs de Paris, Il n'est pas rare dans 381 .

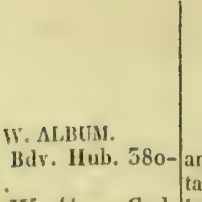

Envergure, 35 mill. - Siles un peu
anguleuses, d'un noir brun, avec une dạn

Environs de Paris,
Ians les bois, les ave-
hues d'ormes. Fin de

|sépara du Lynceus; mais| nous attendrons pour l'imiter la découverte de sa chenille.

tache costale oblongue d'un brun mat, dues d'ormes. Tin de mes; sa chenille est fa pl. 9. fig. 5 et 9 tert. rement deux) point fauve. Dessous d'un de juillet. seule manière d'avoir liğ, 2. brun beaucoup moins foneé que le des- Chenille d'un vert le papillon bien frais; d. Porte-Queue brien d sus, avec une ligne blanche, droite, bien clair, on jaunatre, rare- on se la procure en battaches aurores. 72 A. marquié, s'arrêtant sur les supérieures ment d'un brun clair, tant les ormes. Elle vaB. C. bis. Engr. aux deux tiers de l'aile et formant pris avecle sillon dorsal plus ric beaucoup, et nous de l'angle anal des inféricures une espice foncé et formant de en avons irouvé qui de W trés-anguleux. Ces dernierres petites crêtes; une séric araient le sommet des marquées d'un rang de taches d'un fauve de traits obliques plus crêtes légèrement teinté assez vif, presque toujours contiguës, foncés et souvent unc de rouge-brun, même bordées de noir supérieurement et ligne latérale de la dans le jeune age,

dont les anales appuyées sur de grosses même couleur; tête taches noires.

brune; elle prend

q Dépourvue de la tache costale des une coulcur. rougeatre premières ailes et moins vire en des- quand clle approche de sous.

sa métamorphose. Vit sur l'orme en mai.

Chrysalide d'un brun

clair, pulescente, avec

l'enveloppe des ailes

plus foncée. Sous les

feuilles ou sous l'écorce.

PRUNI.

Lin. Fal, Ochs. Bdv.

llub. 386-58\%.

Envergure, 34 mill. - Ailes un peu Centre et est de la

Ses localités sont asarrondies, d'un brunnoirâtre, avec une France, Sucile, IIon-sez restreintes en Fransérie anté-marginale de taches fauves gric, etc., etc., dansles cc. Il est commun à Poly. du Prunier. God. manquant souvent aux supérieures. bois. En juin. Bondy et dans la forêt pl. 9. fig. 2. Dessous d'un brun jaunêtre, arec une Chenille ayant la de Wolckam, oit hous LePorte-Queue brun a ligne blanche atteignant presque le partie antéricure tris-l'arons pris alsondamlignes blanches. Engr. bord interne aux supéricures; inférien- aptatie, verte, avec une ment. Sa chenille est res avec une large bande anté-margi- ligne latérale jaune, un fort rare. Elle est renale fauve, marque dans toute sa lon- rang de lignes obliques marquable, ainsi que la gueur d'un double rang de points noirs et le sommet des crêt ıs suivante, par l'aplatisdont les supérieurs surmontés d'arcs de même couleur, ces sement de ses premiers blancs.

dernières légèrement anneaux, formant un

q Ayant la bande faure du dessus bordées de brun; tête écusson qui recouvre des ailes supérieures plus prononcée. jaune, arec deux points la tête.

noirs. Vit en mai sur le

pruncllier (Prunus spi-

nosa), l'épine rinette

(Berberis rulgaris), le

chêne, le bouleau, etc.

Clirysalide courte, it

tête rentlée, brune, avec

la partic antéricure

BETULA:

Lin. Fab, Ochs. Bdy.

lïub. $585-585$.

Poly. du Boulcau. God.

pl. 9. fig. 1.

Le Porte-Queue d bande

faites. Enimi.

Envergure, 56 mill.-Ailes d'un brun Dans toute l'Europe, Il n'est pas rare et noirâtre, supérieures avec un trait dis-bois, jardins, parcs. voltige dans les jardins coüdat noir éclairé de jaundète; inférien- En anot et septembre. autour des pruniers. d res ayant i l'angle anal denx ou trois Chenille, ayant aussi une assez grande élétaches fauves. Dessous d'un jaune bru- la partie antéricure trés- vation. On le prend nâtre, les supérieures arec un trait dis-laplatie, d'un vert un souvent fané. Sa checoìdal biun et une ligne blanche; les peu jaunâtre, avee une nillen'est pascommune, inférieures avec une large bande mé- ligne latérale, le som- et nous ne l'arons troudiane d'un jaune plus vif que le fond met des crêtes et deux rée qu'une seule fois et bordée de deux lignes blanches rangs de traits obliques en battant des buissons:

- Aous devons observer que dans toute cette division les taches faures du dessus des o sont tris-sujettes a varier en s'itendant plus ou moins et mene:

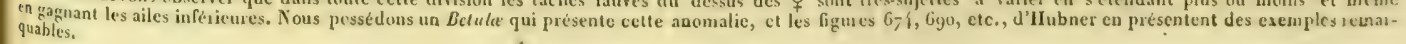

I. ?. 
Jombrées de brun, dont l'interne moitielséparés par des points de prunellier; sa croisplus courle; ces dernières ailes ayant d'un jaune serin ou sance est trés-lente et en outre quelques taches anté-termi- blanchâtre. Vit en juin elle est assez délicate. nales peu arrêtées, fauves. et juillet sur le bouleau

o Beaucoup plus grande (40 mill.) (Betula alba), le pruavec une large tache réniforme et bien nellicr (Prunus spinosa) arrctee, d'un fauve vif sur les supéricu- et le prunier domestires et le dessous des inférieures bien que $(\boldsymbol{P}$. domestica). plus virement coloré que le mâle.

Chrysalide d'un brun roussâtre assez foncé et tiqueté irrégulièrement de noirutre.

\section{(71) Genre IX. HAMEARIS* (HaMEARide).}

(Hub. Curtis. - Melitca. Fab. Ochs, - Argynnis. Lat. God. - Nemeobius. Stephens.)

Caractères principaux. - Chenille onisciforme, courte, hírissece de poils fins. - Chrysalide aussi couverte de poils, renflec sur l'abilomen el "u peu aiguê postericurement. - Palpes ne depassant pas la tête, de trois articles distincts, le deuxieme trés-long, le der nier couri et ovoide. - Antennes assez longues, minces et termines par une massue abrupte et aplatie. - Yeux pu. bescents. - Abdomen plus court que les ailes inferieures, celles-ci ayant au bord abdominal une goullière peu prononcei

Caractères secondaires. - Taille potitc. - Ailes supéricures aiguës au sommet et triangulaires; infírieures un peu aigrtés äl'angle anal ct ligì rement dentes, les quatre brunes avec des taches fauves.

LUCINA. $\quad$ Envergure, 50 mill. - Ailes brunes, Dans une grande par-| Cette espice cst comLin. Fab. Ochs. Bdv. avec des taches fauves, dont celles de tie de l'Europe, bois hu- mune dans certainsbois. Ilub. 21-22. la séric anté-terminale marquées chacu-mides et découverts. En Elle présente une variéArgyn. Lucine. God. pl. ne d'un point triangulaire noir; les supé- mai et aout. té entièrement d'un fau4 quart. fig. 3 et 4 rieures aiguës ì l'angle apical, marquées. Chenille d'un brun ve pâle, avec des taches quint. fig. 5. à la côte de deux points d'unblanc jau-

J.e fauve à taches blan- nfitre de deux sies de ches. Engr.

\section{outre l'anté-marginale; les inféricures} n'en ayant qu'une peu prononcéc. Dessous des supéricures d'un jaune fauve, avec des taches noires et d'autres plus clai. res que le fond; dessous des inféricures d'un fauve plus foncé, avec deux séries de
taches d'un blanc jaunatro et une série taches d'un blanc jaunatre et un

o Plus arrondic et plus marquée de faure en dessus. gne dorsale plus foncée, vit très-cachée, se con-
sur. laquelle se voit a tracte en boule au moinchaque incision une ta-dre attouchement et resche noirâtre, et des sé- te long-temps en cet ries de petits tubercu- état. Elle varie beaules roux sur lesquels coup. it ses différents sont placés des aigrettes âges.

de poils; pattes d'un brun ferrugineux; têtc lisse et ferrugineuse. Vit en juin et septembre, sur la primevère (Primula officinalis) et differentes espèces de Rumex.

Chrysalide d'un brun jaunâtre, velue, et parsemée de points noirs.

- Linnique espece europienne de ce genre, autrefois comprise dans les genres Argynnis ou Mclitaca, est maintenant bien reconnue appartenir au geme Erycit. d. Latreille, tant sous l'etat de chenille et de chrysalide que sous crlui d'inscete parfait; mais ce genre Erycina est loin d'èlre homogene, et prisente une futh d'especes qui ont furt peu d'affiniti: entre elles. Nul duute done qu'il u'ait besoin d'ètre divisé, et Latreille l'a senti lui-mème (Encycl. pag. 555 ); mais, comne est presque entierement composé d'exotiques, les auteurs moderncs s'en sont peu uccupés, et d'ailleurs il est indispensable, pour arriver a une division bien natı relle, d'en connaitre les cbenilles. En attendant nons n'avons cru pouvoj micux faire que d'adopter un genre pour la seule espèce de celte section qu'un trom en Europe, et l. nom d'Ilamearis exislant deja consacré par deux auteurs, nous nous summes empressés de l'adopter pour éviter foute innoration. II virnt probj blement du verbe Auseow, démembrer. 


\section{(72) Tribu II. NYMPIIALIDI (NYMPIALIDES).}

(Lat. God.-Penduli, Bdr.)

Caractères généraux. - Chrysalide fixic par la queue sculcment, et la tête en bas. - Les doux pattes anterieurcs plus courtes ct ne servant point a la marche, au moins dans les máles.

(-3) Les deux pattes antéricures servant à la marche, chez la femelle. . . . . . . . . . . . . . . (

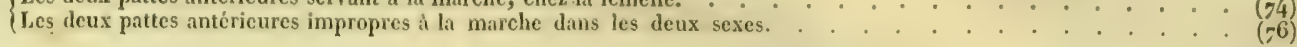

(54) Les deux paties antérieures servant d la marche chez la femelle.

(75) Genre X. LIBYTHEA (LiBythéE).

(Lat. God. Bdv.-IIecaerge, Ochs.)

Caractères principaux. - Chenille légèrement pubescente non ipineuse * - Chrysalide terminée antérieurcment par une pointe mousse. - Palpes formant un bec tris-prolongd et dépassant la tête de cinq ou six millim. - Antennes médiocrement longues et grossissant insensiblement de la base au sommet.

Caractires secondaires. - Taille moycnne. - Ailes supérieures très-anguleuses, a fond brun; inferieures dentées.

GELTIS.

Fab. 0chs. Bdr.

IIub. $447-449$.

Lib. du Micocoulier.God.

pl. 6 r. fig. 5 .

L'Échancré. Engx.

\{Envergure, 43 mill. - Ailes d'un| Mlidi de l'Europe. En) Elle est commune brun un peu jaunatre, chatoyant; les mars et juin. supérieures très-anguleuses, avec six ta- Chenille d'un vert un plus méridionaux de la ches fauves, dont trois réunies, savoir, peu jaunatre et finement France. Il faut frapper une triangulaire ì sa base, une carrée pointillée de blane oulégèrement les arbres près de la côte et une irrégulière et de jaune, avec une li- pour en faire tomber la beaucoup plus grosse sur le disque, ei gne latérale d'un jaune chenille, qui se suspend trois autres, dont une au bord interne pale sur laquelle sont les par un fil, comme celles pariois fondue avec celle du disque, et stigmates bruns et très- des Phalénites. MIM. deux apicales isolées; Ia supérieurc petits; tête et pattes Duponchel, Godart, d'un fauve plus clair. Inférieures trés- vertes. Hubner, etc., ont décrit dentées; avec deux taches également Vit en arril, mai et ou figuré sous le nom fauves, dont la supérieure beaucoup juillet, sur le Micocou-de Celtis une chenille plus petite. lier (Celtis australis). qui ne se rapporte au\& Ayant les taches d'un fauve plus $\begin{gathered}\text { Chrysalide grisatre ou cunement di celte espè- } \\ \text { ferrugineuse, avec deux } \\ \text { pâle. }\end{gathered}$
ce. Notre description est sérics dorsales de points faite sur l'Íconographie noirâtres, et quelquefois de M. Boisduval. entièrement verte.

(-6) Les deux pattes antérieurcs plus courtes el impropres a la marche dans les deux sexes. . .

Chenilles ayant sur le corps ou sur la tête des appendices en forme d'épines ou de tubercules, velus ou grlabres, durs on

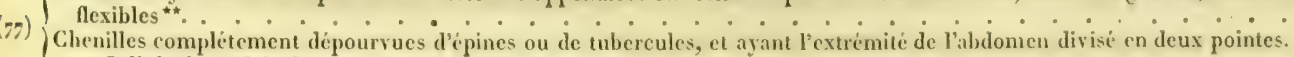

- Cellule discoidale des secondes ailes tonjours fermée.. . . . . . . . . . . . . . . . (96)

(-8) Chenilles a) ant sur le corps ou sur la tete des appendices en forme d’ípines ou de tubcrcules velus ou glabres, durs ou pexibles. (79)

(79) (Epines glabres, molles, flexibles et presque filiformes, - Cellule des secondes ailes frrméc. . . . . . . . . (80) "**

(79) Epines velues ou branchues, de consistance assez ferme - Cellule des secondes ailes ouverte. . . . . . . . . (8s)

- Cette chenille, ricemment découverte par M. Daube de Montpellier, a beaucoup d'afiniti arec celles des Pieris et des Gonopteryx. La clarysalide s'en rapproche aussi un pen, ct l'insecte parfait a, au moins par un des sexes, un grand rapport arec les l'apilfonides. Ce genre ne nous semble done pas pouvoir se placer ailleurs qu'à leur suite, puisqu'il participe à la fois de leurs caractères et de ceux des Nymphatides.

"A l'exception du genre banais, toutes les espèces européennes de cette section ont la cellule discoïdale des secondes ailes ouvcrte, ce qui les fera irès-bien dis-

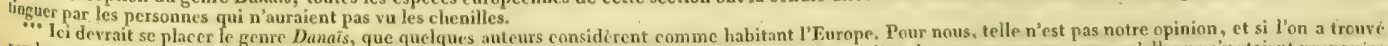

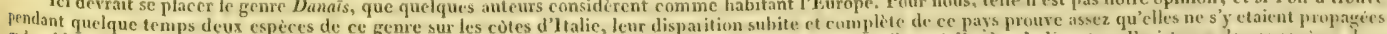
qu'accidentellement. Ce genre a d'ailleurs trop peu d'affinité avec ceux d'Europe pour qu'on puisse altribuex a l'ordre de la nature l'existence de ces cspéces ilans nos contries. Au reste nous en donnons la description, ainsi que les caractères du genre, à la fin de ce rolume. 


\begin{tabular}{|c|c|c|c|c|}
\hline Genre & $\begin{array}{l}\text { Charuxes. } \\
\text { Apatura. } \\
\text { Limenitis. } \\
\text { Vanessa. } \\
\text { Araynis. } \\
\text { M clitar. }\end{array}$ & ? & $\dot{:}$ & \\
\hline
\end{tabular}

(83) Genre XI. CHARAXES (chanaxe).

(Ochs. Bdv. - Paphia, Fab. Dup. - Nymphalis, God. Lat.)

Caractères principaux. - Chenille grosse et courte, ayant le corps sans aucun tubercule, la tete armic de quatre cornes churnues, et le parti postériture échancrie et formant deux pointes peu saillantes. - Chrysalide al angles tris-obtus. - Palpes dépassant lo tête, presque concergents d leur cxtrémite, leur dernier arlicle court et presque nu. - Antennes fortes, asse: longues, massue grossissant insensiblement; goultiire abdominale très-prononcie ef relue.

Caracteres secondaires. - Taille grande. - Ailes superieur's ayant le bord marginal sinué, infirieures munies de deux queues pris de l'angle anal.

\begin{tabular}{l|l} 
JASIUS. & Envergure, 78 mill. $-\Lambda$ iles d'un brun
\end{tabular} Lin. Fab. Ochs. Bdr. velouté; les supérieures arec une borGod. pl. 10, fig. 3-4. dure jaune surmontée d'un rang de taRhea. Hub. 111-112, clies semblables, les inférieures glacées 580-581. et Unedonis de verdâtre, avec une bande anté-termi(la chenille).

\begin{abstract}
nale jaune, surmontée près de l'angleanal de quatre a cinq points bleus et les queues brunes, l'antérieure plus courte: dessous ayant sa première moitić d'un brun-rouge, arec plusicurs taches noires bordées de blanc, et séparíes par une bande transverse d'un blane satiné de la seconde moitié, qui est grisâtre, et lesbandes jaunes du dessus; inféricures ayant à l'angle anol une seconde bande jaune, courbe et formant une espèce d'cil noir bipupillé de violet.
\end{abstract}

† Semblable, mais plus grande.
Lspagne, Portugal, Il est maintenant bien Provence, îles d'uJières, répandu dans les collecenvirons de 'Toulon et tions. II plane en volant. de Montpellier. En juin et aime at se poser sur et septembre. les fruits pourris; nous Chenille plate en des- l'arons pris ainsi à Mont. sous et renflée au mi-pellier où.il est rare. Il licu, d'un vert jaunatre, exhale en mourant une ar ec une ligne latérale odeur de muse très-projaune au-dessus des pat- noncée; la bande jaunc tes, et deux taches ocel-des ailes inférieures esi lées d'un vert jaunâtre, teintée de verdâtie en a pupille bleae sur les se rapprochant de l'anseptieme et neuvieme gle anal; les taches anneaux; tête verte, blenesquilasurmontent avee quatre cornes épi- manquent quelquelois. neuses jaunâtres, ayant le côté cxtérieur et le sommet rougeâtres, Vit en mai et noot sur l'Arbutus unedo.

Chrysalide grosse, ovoïde, lisse, d'un vert pále, arec deux pointes ì sa partie postéricure.

\section{(84) Genre XII, APATURA * (apature).}

(Fab. Ochs. Bdr. Dup. - Nymphatis. Lat. God.)

Caracties principaux. - Chenille presque rase, renflec au milicu, aplatic en dessous, atec la tite plate ct surmontic de deux cornes ipincuses, la partie postérieure fendue et divisée en deux pointes prolongées. - Chrysalide bombie sur le dos, arec la tcle bifude d aiguë.-Dalpes plus longs que la tête, peu velus, convergents a leur extrimité, leur dernicr article très-courtet presque nu. Antennes assez forles, longues, ì massue grossissant insensiblement; goutlicre abdominale tris-concatc.

\footnotetext{
- Quvique d'un facies bien différent de celui des Charaxcs, ce genre s'en rapproche beaucoup si l'on ne considère que les palpes et les antennes; mais nous ne whardons pas les caracteres tirés de ces parties comme exclusifs, et les Apaturn sunt si differentes des Charaxcs a l'état de cluenille el de chrysalide, que nous

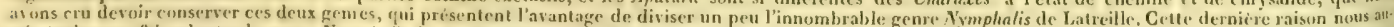
r.tit egalement fait adupter le genre Nymphalis de M. Boisdnval, gui differe un peu des Limenilis par la bouclic ; mais la chenille de l'unique espere curopienue

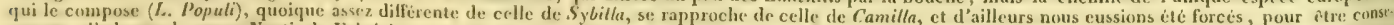

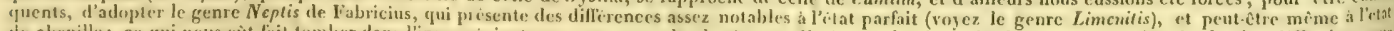

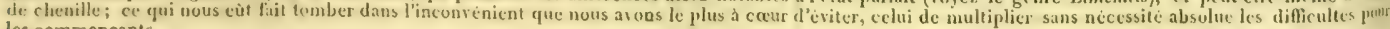
les commençants.
} 
Curnctèren secondiures. - Taille grande ou au-dessus de la moyenne. - Ailes supérieures sinudes; inférieures denties, sans queue, les quatre
ornées de taches oculuires; un reflet violel trís-vif dans les mâles.

ILIA.

Fab. Ochs. Bily.

Envergure, 6o mill. - Ailes d'un

Dans une grande par-1

brun noir, arec un reflet violet tris-vif;

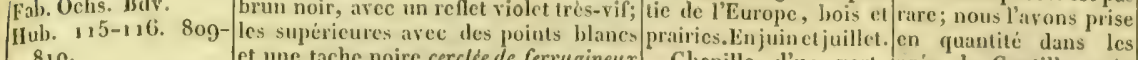

810.

et une tache noire cerclie de ferrugineux Chenille d'un vert-prés de Gentilly press God.pl.6quert. fig. 2. près du hord marginal; les inférieures jaunatte sale, arec plu- Paris, et dans les en-
Le petit Mars chan- avec une bande transverse blanche, di- sicurs lignes obliques droits bas et humides de geant $(62$ e. d. et 6 亿 visée en taches par les nervures; la ta- jaunatres, dont l'inter-plusieurs bois; elle aime e.). Eugr. che de la cellule ne dépassant pas les au-médiaire saillante sur le à se poser sur les feuilles tres et un oeil cerclé de ferrugineux à dlos et deux longitudina- ou contre le trone des l'angle anal; dessous d'un gris jaunatie, les sur le cou; tête an- arbres; les femelles voles supérieures lavées de faure al l'angle guleuse, avec deux lon-lent sourent très-haut, apical, les inféricures arec la bande gues épines fouichues mais elles descendent transverse d'un blanc violatrc nullement au sommet, vertes, bor- vers trois ou quatre ombrée extéricurement, et un ou deux dées de jaune, et ayant heures de l'aprés-midi, points noirs a la base. flet.

sur leur côté antérieur et sont alors faciles it o Plus grande, plus claire et sans reune ligne noire; pattes saisir.

vertes. Viten mai et juin,

sur plusieurs espèces de saules et de pcupliers, au sommet desquels elle se tient.

Clirysalide d'un vert pâle, avec la carène du dos, les deux pointes de la tête et le bord de l'enveloppe des' ailes d'un jaune clair.

Civitie. Hub. $185-114$. tous d'un jaune fauve clair, d l'exception Ilia. rar. Ochs. Bdr. des trois points blancs apicaux des suGod. pl. 6 quart. péxieures, qui restent blanes; en outre fig. 5 .

Le petit Mars orange. (64 c. d. f. g. 66 a. même jaune (dont on ne voit que les b.). $\operatorname{ling} r$.

LE GRAND MaRSORANGÉ. Engr. 63 a. b.

Metis. Kindermann.

Ilis.

Lin. Fals. Ochs. Bdy.

Sous-rariété, femelle, dontle fond est entierement jaune, arec quelques taches brunes et les bandes transverses d'un jaune plus clair que le fond. supérieures une tache également jaune, marquée de quatre points noirs; le fond est d'un brun moins noiratre et le reflet est d'un violet plus rosé.

o Inalogue.

IIul. 11 $=-118.584$.

Diffère de Clytie par le ton plus rougeâtre du fauve, par le reflet plus foncé et plus éclatant, et par l'absence des yeux d̀ l'angle anal des inférieures.

Envergure, zo mill. - Ailes d'un quatre ailes une bande maculaire du

Mêmes localités.

Cette rariété, quoique bien différente au premier coup d'œil, provient de la même chenille qu'Ilia et n'est pas plus rare dans nos pays. Dans le midi on la trouve seule, et elle y parait en juin et aoat; ce qui ferait supposer, dit M. Duponchel, qu'elle a deux pontes par an. On cite des bybrides qui sont llia à droite et Clytie it gauche, et d'autres Clytie en dessus et Ilia en dessous.

On la trouve avec les femelles ordinaires, mais plus rarement. Nous l'avons prise aussi à Gentilly.

Syrmic, France mé- M. Treitschke a vu des individus où les yeux commençaicht it reparaître, et dont la couleur se rapprochait des Clytie ordinaires. Dans une partie de
Elle est plus rare qu'Ibrun noir, aree un reflet violet tries-rif; l'Europe, principale- lia dans nos environs.
les supericures aree des points blanes et ment vers le nord, dans Nous l'avonspriseabonune tache noire (tri-s-arement cerclé les grands hois. En juin damment dans lat forêt de Mlormâle, ar ecla Lim. 
gean $o^{7}$ et le grand les inférieuresarecune bandetransverse Chenille d'un vert|populi. Les femelles II urs non changeant blanche, divisée par les nervures en ta-jaunatre, avec plusieurs sont beaucoup plus diff. $(62$ a. b. 65 a. ches dont celle de la cellule forme une lignes obliques jaunat- ficiles a obtenir que les b.). Engr.

cerclí de ferrugineux a l'angle anal. saillante sur le dos et Dessous d'un grís un peu rosé; les su-terminée pardeux points périeures ayant à l'angle apical une bleus; tête plate, angugrande tache d'un rouge brun neltement leuse, arec deux épines coupee; les inféricures a vec labande trans- fourchues vertes, borverse d'un blanc pur, largement ombrée dées de jaune et ayant de rouge-brun du côté externe.

sur leur cóté antérieur f Plus grande, plus claire et sans reune ligne bleuâtre. Vit en mai et juin sur le peuplier et le tremble, au haut des branches. Chrysalide ayant de plus que celle d'Ilia quelrgues bandes obliques jaunatres sur les côtés.

Fab.

Benor,

Iole. Hub, 622-625. et Iris. 78 4 -785 . Iris. var. God. Bdy.
Differe de l'Iris en ce qu'elle n'a sur les supérieures que les taches blanches de l'angle apical, et qu'il y a absence totale de la bande transverse aux inférieures, ou l'on voit seulement quelques poils blancs,
Celte variété n'est pas constante, et l'on rencontre sourent des individus plus ou moins privé de taches et bandes blanches sans l'être pour cela complétement. Nous n'avons jamais vu de femelles offrant la même anomalie; cependant la fig. $65 \mathrm{c}$. d. d'Engramelle paraît en être une.

\section{(85) Genre XIII. LIMENITIS (LIMínIte).}

(Ochs. - Limenitis. Dalm. - Limenitis et Neptis. Fab. - Limenitis et Nymphalis. Bdv. - Nymphalis. Lat. God.)

Caracteres principaux. - Chenille garnie d'e pines rumeuses ou de tubercules velus d'inégate longucur. - Chrysalide ayant sur te dos une bosse tris-saillante et la tête munie de deux tubercules plus ou moins longs. - Palpes dípassant un peu la tête, un peu écartés, velus; leur dernier article tréswcourt, un peu velu et terminé en pointe. - Antennes longues, a massue grossissant insensiblement.

Caractires secondaires. - Trille arande ou moyennc. - diles dentes, brunes, avec des bandes de laches blanelies el les échancrures de la même couleur; point de reftet ni de taches oculées.

Chenille à tubercules épais, munis de poils dont l'extrémité est en massue, à tête bifide. - Chrysalide terminée antérieurement-par deux pointes obtuses. - Niles subtriangulaires, les supérieures sinuées au bord terminal, les inférieures un peu prolongées à l'angle anal. - Palpes courbes.- Intennes longues et fortes; gouttière abdominale très-prononcéc et ne portant pas l'empreinte des bandes blanches.

Chenille it épines rameuses ou it tubercules épais, munis de poils simples et à tête cordiforme. C Chrysalide termince antéricurement par deux cornes ou oreilles plus ou moins longues. - Ailes et gouttière abdominale comme dans la division précédente. - Palpes presque droits, assez forts, ainsi que les antennes. - Corsclet robuste. . . . . . . .

Chenille et chrysalide...... - Palpes et antennes grêles. - Corselet assez étroit. - Ailes supérieures olılongues, arrondies au bord terminal, ainsi que les inférieures, qui ne sont nullement prolongées it l'anghe anal; goullicre abdominade peu prononcée et portant l'empreinte des bandes blanches.

(8-) Chenille à tubercules épais, munis de prils dont l'extrémité est on massue, à tête bifide. - Chrysalide terminée antéricurement par deui pointes obtuses. - Ailes subtriangulaires; les supérieures sinuées au bord terminal, les inférieures un peuprolongées à l'angle anal. Palpes courbes. - Antennes tongues et fortes; gouttiere abdominale tris-prononcie et ne portant pas l'empreinte des bandes blanches.

\section{Genre NYMPIIALIS, Bdv.}

POPULI. Enrergure, zo mill. - Liles d'un| Europe septentrionaCette belle espece se Lin. Fab. Ochs. Bds. brun noiratie; supérieures aree le bord|le, dans les grandes fo-prouve ga et lia, dans les| 


\section{G. LIMENITIS.}

Llub. 108-110. |terminal longé par deux lignes macu-|rêts. Dans les premiers/grandes forêts de nos| God. pl. 6. fig. 1-2. laires plus foncées, et éclairées de faure jours de juin. Le grand Sylvain et le au sommet de l'aile, et des taches blan- Chenille d'un vert très-commune dans le Sylcain. Engr. ches dont les intermédiaires en forme jaunâtre, avec les qua- nord, surtout dans la fodebande très-sinueuse; inférieuresavec tre anneaux intermé- rêt de Mormale, ou nous une bande médiane de celte couleur, in- diaires d'un, rouge vio-l'arons prise abondamterrompue par les nervures, et le bord latre pale, et deux lunu-ment. Elle aime a se poterminal, glacé de verdatre, divisé par les noires superposées ser sur la fiente des bes. une ligne noire et surmonté $d^{\prime} u n e$ ban- sur le cinquième an- tiauxetdaos les endroits de fauve maculaire placéc entre des lu- neau; épines plus lon- humides des allées des nules noires; dessous d'un fauve jaunâ- gues sur les premiers bois. Il faut se garder de tre, avec lestaches du dessus un peutein- anneaux; tête d'un roux la poursuirre si on la tées de verdattre; supérieures ayant le ferrugineux, avec les manque, car elle revient bord interne largement noir; inferieures côtés noirs; pattes fer-d'elle-même au même avec le bord abdominal, le bord termi- rugineuses. Vit en mai, endroit. La femelle est nal et une tache à la base d'un gris ver- sur les Populus, alba et plusrareque le mâle, vu dâtre pâle, et un rang de points noirâ- tremula, â la cime des-qu'elle se tient an somtres sous sa bande blanche.

$\&$ Un peu plus grande, plus arrondie, avec les taches blanches plus étendues.

Tamaule.

Les taches blanches des supéricures Populi. rariété God. sont presque toutes très-saupoudrées de Ochs.

quels elle se tient. mel des arbres et des-

Chrysalide jaunatre, cend rarement id terre.

nuancée de roussâtre et

ponctuéc de noir.

Mêmes localités.

Cette variété, correspondant à la var. Beroe de l'Iris, se rencontre brun, et la bande blanche des inférieures manque totalement.

(88) Chenille dipines rameuses ou de tubercules épais, munis de poils simples, et à tête cordiforme. - Chrysalide terminée antericurcment pur deux cornes ou orcilles plus ou moins longrues. - Ailes et gouttiere abdominale comme dans la dicision pricédentc (87). - Palpes presque droits, assez forts, ainsi que les antennes. - Corselet assez robuste*.

\section{Genre LIMENITIS. Bdr. Dup.}

CAMILLA.

Fab. Ochs. Bdy. Ilub. $106-107$. Fod, pl. 6. fig. 3. et 6 tert, fir. 2.

Le Sytoain azure. Engr.
Envergure, 53 mill. - Ailes d'un noir Centre et midi de la| On rencontresouvent bleit, avec une bande transverse blan- France, dans les parties des incliridus qui sont che, maculairc aux supéricures, coupée bumides des bois. En marqués de légéres tapar les neryures aux inféricures, et une mai, juin, juillet, aoot, ches rouges it l'angle séric anté-terminale de points noirs éclai- suivant les localités. anal desinférieures; cerés de bleualre; supéricures ayant en ou- Chenille d'un. beaula arrive surtout chez tre trois taches blanches, dont une dans vert, avec le ventre et les femelles. Cette Lila cellule précédée d'atomes bleuâtres; les pattes d'un rouge ro-ménite est moins répandessous d'un noir-brun varié de rouge- sé, et garnie de gros lu-due que la sibylla el brique, arec les taches du dessus; infé-hercules verts ì sommi- n'habite pas le nord. On rieures ayant la base et le bord abdomi- té rougre, velus, inégaux la rencontre sourent au nal largement bleuâtre, avec deux si- et plus grands sur le bord des ruisseaux et gnes noirs et une série anté-terminale cinquième anneau; tête dans les lieux humides; de points noirs renformés chacun entrc rouge. Vit en avril, mai cependant nous l'arons deux taches $d^{2}$ un rouge-brique.

o Plus grande et à taches blanches feuille des bois (Loni- secs et élevés. Pour se plus marquées.

SIBYLLA. Lin, Fab. Ochs. Bdr. llub. $103-105$. fiod. pl. 6 sec. fig. 3 . e 6 tert. fig, 1. - Il cst i remarquer que dans ce genre et les deux precedents les pattes antericures, quoique plus courles que les autres et impropres a la marche comme dans

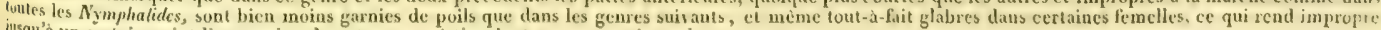
fusqu'à un certain point l'expression de pattes cu palotinc dont se sont serris quelques auteurs. 
I.e petil Sylcain. Engr. foutre unc tache blanche trés-saupoudrée|membraneuses plus pâ-|du même genre. Elle! de brun dans la cellule et deux ou trois les, et une ligne latérale varie beaucoup pour la autres derrière la bande; dessous d'un blanche au-dessus des taille, mais peu pour les fauce ferrugineux, avee les taches du paltes; elle est garnie couleurs; cependant les dessus; inferieures ayant la loase et le d'ćpines ramenses peu bandes blanches s'étiobord abdominal d'un blanc bleuatre, et épaisses et assezcourtes, lent parfois en partic trois séries anté-marginales de points d'un rouge violâtre ou comme chez la $L$. popunoirs, dontquelques-uns fortement iclai- ferrugineux, pattes i- li. On rencontre trèsrés de blanc près de l'angle anal. cailleuses de cette cou-souvent des individus

+ Plus grande, plus arrondie, à ta-leur; tête d'un roux pâ-femelles et quelquefois ches blanches mieux marquées, et at le. Yit en mai, sur le des males qui sont marbande transverse des supéricures non Lonicera periclymenum. qués à l'angle anal des interrompue.

Dans les endroits humi- inférieures et d̀ l'angle
des des bois.
Chrysalide brune,
apical des supéricures
dequelques taches rous-
vec une petite bosse
très-saillantesur le dos,
qui est orné de taches
d'or et d'argent, et la
tête munie de deux cor-
nes ou oreilles très-lon-
gues.

(89) Chenille et chrysalide..... - Palpes et antennes grêles. - Corselet assez étroit. - Ailes supéricures oblongues, arrondies au bord terminnt, ainsi que les inferieures, qui ne sont nullement prolongées d l'angle anal; gouttiere abdominale peut prononcée et portant l'emprcinte de! bandes blanches.

\section{Genre NEPTIS. Fab.}

LCEILAR.

Fal. Oehs. God.

Hub. $101-102$.

Dup. Suppl. pl, 16. fir. $1-2$.

Bdv, Icon. pl. 18. fig. 1.

Le Sylrain canobite. Engr.
Envergure, 52 mill.-Ailes d'un brun| IIongrie, Styric, Piénoir, avec des taches blanches; supé-mont, Autriche, Suisse, rieures aree un petit trait blanc à la base, lissie. En juin et juillet. surmonté de trois points dont celui du milieu plus gros, puis une bande maculaire trís-irrígnliere, composée de taches de diverses grandeurs; inféricures avec une seule bande transwerse, maculaire et attrignant te bort abdominal. Dessous d'un ferrugineux fonce, avec les taches du dessus; inférieures ayant en outre une bande courte hasilaire et une douhle série anté-terminale de lunules grisâtres.

o Semblable, mais plus grande.

ACERIS.

Fal, Ochs. God.

Envergure, 47 mill. - Ailes d'un Autriche, IIongrie, brun noir, avec des taches blanches dis-Servie, Russic, Mora-

Bdr. Icon. pl. 18. fig. 2. posées par bandes; supérieures en ayant vic, etc., dans les bois.

Lim. de l'Erable. Dup. une longitudinale partant de la base, en Ln juin.

Suppl.pl. 16. fig. 3-4. forme de fer de lance interrompu près

$P$ latuilla. Hub. 99-100. du sommet; les quatre ailes ayant en

Le Sytrain di deux bandes outre chacune deux bandes, dont l'interblanches. Engr.

ne des inférieures continue et les autres maculaires. Dessous d'un rouge ferrugineux, avec toutes les bandes du dessus, et de plus trois lignes blanehes a chaque aile; celles des inférieures, dont la côte est anssi blanche à la base, alternant avec les bandes du dessus, et celles des supérieures disposées, savoir, une le long de la côte, et les deux autres non loin du bord terminal.

f Plus grande.
On observe souvent sous les ailes supéricures quelques taches blanches entre la côte et la ligne basilaire. Cette Liménite est rare. On ne la trouve en Suisse que dans la partie la plus méridionale du canton du Tessin, près de Lugano. Elle vole dans les forêts de châtaigniers.

Elle áime ì voltiger autour des pruniers. Elle n'est pas commune. On la trouve aussi dans quelques parties de I'Asie, mais elle est alors généralement plus grande qu'en Europe.

Nola. Outre la loule de caractères qui séparent ces deux especes des précédentes, on les en distinguera encore facilement en ce que la base et le bord interne des inféricures sont concolores en dessous, au lieu d'être blenâtres. 


\title{
(90) Genre XIV. VANESSA (Vanesse).
}

\author{
(Lat. God, Ochs. Bdr.-Aglais. Dalm.)
}

Caractères principaux. - Chenille churgée d'épines velues ou rameuses et d'egule longueur. - Chrysalide terminic anterieurement par deux pointes, et marquée de taches dorées cu argentees. - Palpes vclus, moitié plus longs que la tête. - Antennes longues, d tige assez mince, d massue brusquement renflee ct non crcusée ni aplatie en dessous. - Corselet gros el fort, - Abdomen

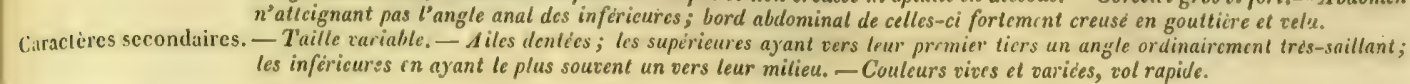

pronsi.

Lin. Fab. Ochs. Bdv. II ub. $94-9^{6}$.

God. pl. 5 sec. fig. 5. et 5 tert. fig. 2.

La Carte geographique brune, Engr.

Bir.

La Carte gcographique rouge. Engre.

Lin. Fab. Ochs.

IIub, $97-08,-28-729$ God. pl. 5 sec. fig. 4 et 5 tert. fig. 5.

La Carte géographiquc faurc. lingr.

Prorsa. var. Ibelv. Dup. Icon, des chenilles.

Envergure, 35 mill. - Ailes dentées, IIongrie, Russie, Li-
'un brun noir, arec la frange blanche vonie, France septen-

Des expériences réid'un brun noir, arec la frange blanche vonie, France septen-tirées ont demontré que entrecoupée; les supérieures ayant l'an- trionalc, environs de Va- ce Lépidoptère et les gle du sommet très-arrondi, marquées lenciennes, de Senlis, deux suirants ne sont dans leur milicu d'une bande d'un blanc de Saint-Quentin, de qu'une scule et même jaunatre interrompue au milieu, puis, Paris, etc., etc. Dans les espèce dont la différenentre cette bande et le bord marginal, forêts, sur les ronces ce est produite par l'éd'une séric de points blancs et d'un qui croissent dans les poque de leur éclosion. trait fauve au bord interne; inférieures lieux humides. In juil- Ainsi les chenilles qu'on arec la bande blanche continue et deux let et aont. trouve en juin éclosent lignes anté-marginales fauves. Dessous Chenille noire ou gri-len juillet et aoot ei d'un ronge-brun foncé, arec une mul- sâtre, piquée de blanc, donnent la Prorsa, et titude de lignes blanches, et la bande du arec les épincs noires celles qu'on rencontre dessus; inféricures ayant en outre a l'an- ou jaunes, et les pattes en automne passent l'higle anal et d'angle du milieu du bord membraneuses noires, al ver et produisent la $L_{e-}$ marginal deux petites taches d'un bleu extrémité jaunatre. - vana. On peut même â violet.

o Plus grande, et ayant les lignes fau* ves du dessus plus marquées.

Ligne faure du bord interne des supérieures plus marquée; bande blanche des mêmes ailes teintée de jaune; bande des infërieures fauve et souvent divisée en deux près du bord abdominal; lignes anté-terminales fauves larges et bien plus marquées. Dessous participant de la Prorsa et de la Levana, et se rapprochant tantôt de l'une, tantôt de l'autre.

On trouve souvent une volonté obtenir l'une ou variété qui offre sur les l'autre, en retardant, au côtés une ligne longitu- moyen d'un froid artidinale fauve. Vit en juin ficiel, l'éclosion des preet septembre sur l'ortie mières.

(Urtica dioica). Cette espèce n'est pas Chrysalide anguleuse rare dans le Nord, mais brunâtre ou roussâtre, aux environs de Paris ayant sourent de petites on ne la troure que de taches métalliques. loin en loin.

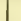
The

Le fond des ailes, au lieu d'être brun, est d'un jaune fauve, parsemé d'un grand
nombre de taches brunes, arec deux ou trois taches costales d'un blanc jaunâtre sur les supérieures, et deux points blancs, dont le supéricur plus gros, placés non loin du bord externe; les inférieures ont une large bande fauve anté-marginale marquée d'une série de taches brunes arrondies, et d'un rang presque terminal d'autres taches brunes lunulées. Le dessous est un peu plus clair, lc jaune y domine davantage; les bandes médianes surtout y sont peu distinctes, ct cellc des inféricures est saupoudrée de brun dins tout son milieu; enfin les quatre
Celte variété, complétement intermédiaire entre Prorsa et Levana, se rencontrequelquefois dans la nature; mais on l'obtient plus facilement en faisant éclore au milieu de l'hiver, et par une chalcur artificielle, des chenilles de Prorsa.

Mêmes localités. En Elle est moins comavril et mai. mune que la Prorsa. Nous l'avons prise plusieurs fois dans la forêt de Mormâle. Elle plane en rolant comme une Limenite, ainsi que Prorsa. 
failes ont a l'angle médian une large tache violatre au milieu de laquelle est un point blanc, et le point de l'angle anal est changé en ligne.

o semblable, mais plus grande.

ANTIOPA.

Lin. Fab. Ochs. Bdv. Hub. $79-80$.

Yan. Antiope. God. pl.

Le Morio. Engr.
5. fig. 1 .

Envergure, zo mill. - Siles veloutées, d'un brun-rouge foncé, arec une bois, prés, jardins. En
large bordure jaune piquée de noir, et une juillet, aoat et septem- bande anté-terminale noire, diviséc parbre. une série de taches bleues; les supérieures ayant deux taches jaunes à la côte, qui est striée de la même couleur; les de inféricures ayant vers le milieu de leur bord externe un angle très-saillant, formant une espèce de queue. Dessous d'un
noir obscur, aree la bordure et un point central d'un blane jaunâtre.

o semblable.

10.

Lin. Fab. Ochs. Bdr. Hul. 77-7

$V$ an. piun au jour

God. pl. 5. fig. 2.

Le Paon de jour. Engr.

Envergurc, 55 mill. - diles anguleuses et dentées, à frange noire. Dessus des supérieures d'un rouge ferrugineux vif, arec deux taches noires et une jaune près de la côte, qui est striéc à sa base de cette derniére couleur, ct i l'angle apical une grante tache acelléc, à prunelle ferrugineuse, cntourée de jaune du côté du corps, et de bleu violet du côté tes pattes membra-chenille vit sur le houdu bord, où on apercoit trois points neuses ferrugineuses. blon : nous ne l'avons blancs, qui forment, avec deux autres Vit en société sur l'ortie jamais trourée sur cette pareils qui sont au-dessous, une ligne dioïque (Urtica dioica). plante, quoique nous l'y transverse; dessus des inférieures poin- On la troure dans la ayons cherchée. Queltillé le jaune à leur base, ayant ì l'angle dernière quinzaine de ques-uns des individus supérieur ure grande tache ocellé, noire, juin et en anot. Elle est qui éclosont dans l'arrièchargée d'atomes bleus, et entouréc fort sujette à être pi- re-saison passent l'hid'un cercle blanchâtre. Dessous des qua- quée par des diptères de ver cachés dans des tre ailes d'un noir-brolé; les inférieures la tribu des Muscides. trous d'arbres, dans des avec une ligne plus foncéc et un point central blanchatre.

Iotors.

)ahl.
\& Semblable au ơ, mais plus grande.

Chrysalide verdatre greniers, etc., et paraisou brunâtre, avec des sent dès les premiers taches dorées, les poin- beaux jour's du printes de la tête et celles du temps. Nous en arons corselet tris-aiguës, et pris volant au mois de deux rangées d'épines décembre $1_{2} 6$, résur le dos.

Ne differe d'Io, suivant MI. Treitschke, que par unc taille moitić plus petite. chaufiés par la chaleur du feu d'une chambre d'auberge nù nous nous trouvions. Il existe dans la riche collection de 11. Marchand une variété complélement dépourvue de tarhes oculies..

Nous n'arons pu nous procurer cette variété; mais M. Treitschkeassure l'avoir élevéc, et n'y avoir remarqué aucune 
ATALANTA. Jin. Fab. Ochs. Bdy. llub. $75-76$. $V^{\prime}$ in. Vulcain. God. pl.

6. 6. 1. l. Vulcain. lingr.

différence dans la chenille ni dans le papillon.

Envergure, 6o mill.-Ailes dentées, Dans toute l'Europe, Cette belle espéce fupé- dans les jardins, dans est fort commune, et se ticures noires, avec une banle transverse les bois, sur les fleurs, Irouve aussi en Barbar'un rouge vif, et une grande tache et plutot cncore sur le ric, en Ligypte et dans blanche placée près de la côte, suivie de trone des arbres qui l'A sie mineure. cing autres en form? de points, dont la laissent suinter leur On nbserve ordinaipremière et la quatrieme plus grosses, sève. rangées en ligue courhe vers le bord, Pendant toute labelle rouge des supérieures qui est légècement nuancé yers l'angle saisun et principalement un point blanc faisant apical de bleu violet; inféricures uni- en automne. formément dentées, noires, arec une Chenille d'un jaune mais ce point manque large bande terminale d'un rouge rif, rerdatre sale, ou d'un assez souvent. Cette essur laquelle est une ligne de quatre cendré vinlatre, cou-péce et la suivante forpoints noirs, terminée a langle anal par verfe d'épines branchue puen une exception une doinble tache bleuatrc. Dessous des jaunes, arec une ou dans ce genre, en ce supérieures parcil au dessus, mais plus deux lignes maculai-que leurs ehrysalides pále, avec quelques traits bleufitres à res et latérales de la ont les angles arrondis leur base et au-dessus de la lyande rou- même coulcur. Tête et que leurs ailes infége, qui est blanchatre à ses extrimités, noiratre et pattes bru- ricures sont uniformél'angle apical grisatre, et deux petits nes. Vit solitaire sur ment dentées et n'ont points ocellés ì son sommet. Dessous l'ortic dioïque (Urtica point d'angle saillant des inférieures brun. marbré de gris et (fioica) et l'ortie griè- vers le milieu du bord cle noir violatre, arec une tache jaunâ- che (Urtica trens), marginal; langle des tre au bord interne, et une rangée de dont elle roule les feuil- supérieures est aussi taches anté-marginales un peu oculées. les pour s'envelopper. nuoins aigu. La forme \& Semblable, mais plus grande. $\quad$ Elle est trés-sujette al de la chrysalide influe êtro piquice par les ich-donc ici fort sensibleneumons. On la troure ment sur l'insecte paren juillet, aont et sep- fiait.

tembre.

Chrysalide d'un gris blanchâtre ou brunâtre, avec les angles arrondis, des taches métalliques, et sur le dos deux rangées de petites épines noires.

CABDUt. Jiin, Fib. Ochs. Bdv. Ilub. $73-74$.

Van. dic Chardon. God. pl, 5 sse. fie. s. Ja Belle-llamr, lingr.

\section{Envergure, 58 mill. - Ailes dentées, Dans toute l'Europe,} 1) Irange blanche lille est trés-commules supúrieures brunes, saupoudrées jardins, champs, etc., ne et répandue sur les suporicures lrunes, saupoudrées sur les chardous. En presque toute la surfatde jounâtre à la base, avec le disque mai, aoot et septembre. ece du globe. Elle est coupé par de larges laches. irrouliè'es Chenille trés-épineu- aisée à prendre, quoid'un jaune fune plus ou moins rosé et se, d'un gris bleuatre qu'clle ait le rol rapide plus vif à la base, et le sommetmarqué ou brunâtre, sale, arec et soutenu, parce qu'on de taches blinchesdontl'interne isolée, quatre lignes jaunes la voit souvent posée large, coupee par les nervures; les sui- sourent confondues. dont à terre ou sur les charvantes au nombre de quatre, dont les deux dorsales et deux dons. Sa chenille est intermédiaires plus petites et les anté- latéra'es. Téte et pattes tantôt rare et tantồ terminales trés-petites; inféricuresbru- brunes. Vit solitaire fort commune. Sa nes, saupoudrées a la base de poils et sur plusicurs espéces chrysalide est encore d'alomes jaunâtres, arec une tache discoi- de chardons, mais sur-plus arrondie que celle dale et une tris-large bande terminale tout sur les C. acunthoi- de l'Aialanta et par suid'un jaune futre un peu rosé, la der- des et nutans. Elle s'en- te ses ailes moins angunière marquéce d'une série de taches reloppe dans un réseau leuses, surtout les surondes, puis d'un rang de point', puis qu'elle file it l'embran-périeures. Nous arons d'une serie de taches terminales noires. chement des tiges, et pris aux environs de Dessous des supéricures d'un rose vif hi ronge le parenchyme Chartres une rariété la base et un pen jaunatre vers le bort des feuilles qu'elle peut dont le dessus des inféinterne, avec les taches blanches et atteindre en sortant a ricures est presque enles parties noires du dessus, mais plus moitie de ce nid. On tierement brun et les contuses. Dessous des inférieures va- la trouye en juin et points ocellés du desrié de brun jaunatre et de blanchâtre. aont. avec une séric de points oblongs sur- Clirysalide a angles grands. Germar figure montés de quatre taches tris.ocelle'cs, tresearrondis, d'unjaune (fasc. VII. Tab. i6.) 
dont les intermédiaires plus petites.|clair ou d'un brun mus-|une autre sarićté ac-

+ Plus grande et ayant les supérieu- sâtre, arec deux rangs cidentelle encore plus res moins aiguës au sommet.

de tubereules dorés sur remarquable.

le dos.

Fn examinant bien cette espece on y retrouve presque tous los caractères de l'A talant", quoique très-modifiés. On roit de même dans quelques femelles un cinquième point blanc placé sur la partic fau-

URTICA:

Envergure, 47 mill.-Ailes dentées, ve des supérienres.

Lin. Fab. Ochs. Bdv. Hub. $87-88$.

Vix. var. 89 .

d'un fauve rourgêtre, arec le hord ter-jardins, prés, bords des commune partout et minal brunatre, coupé d'une ligne noi-chemins, etc. Pendant lu'est point difficile a re et surmonté d'une bande noire char- toute la belle saison. saisir; quelques indivi-

$V$ an. de l'Ortie, God. pl. gée de lunules bleues; supérieures avee Chenille d'un brun dus passent l'hirer ft 5 sec, fir. 1 .

$L$ a petite Tortue, Engr. six taches noires, dont trois costales sé-noirâtre, piquée de rolent les premiers parées par des éclaircies jaunes et sui- jaunâtre, arceuneligne beaux jours, mais en vies it l'angle apical $d^{\prime}$ une tache blanche; dorsale, d'un jaunc ci- maurais état.

les trois autres discoïdales, et dont l'in-tron, divisée dans sa férieure, qui est beaucoup plus grosse, longueur par une ligne éclairée extéricurement de jaune; in- noire et une ou deux férieures anguleuses vers la moitié du raies latérales du même bord externe et ayant la base largement jaune; épines noires ou noirc et éclairée de jaunâtre à la côte. jaunâtres, tête noire,

† Plus grande, avec les éclaircies pattes noirâtres. Vit en jaunes plus larges.

société surquelques espèces d'orties, mais principalement sur I'U. dioica.

Chrysalide d'un gris brunâtre, avec la partic antérieure unpeu dorée ou même entièrement d'un jaune d'or brillant. Le long des toits, des arbres, etc.

ICHNUSA.

Bon. Ramb.
Bdv. Icon. pl, 24, fig. 2.

Envergure, 47 mill.-Ailes dentées,

Corse et Sardaigne. d'un fauve rougeâtre, arec le bord terDup. Suppl. pl. 23. fig. 4.

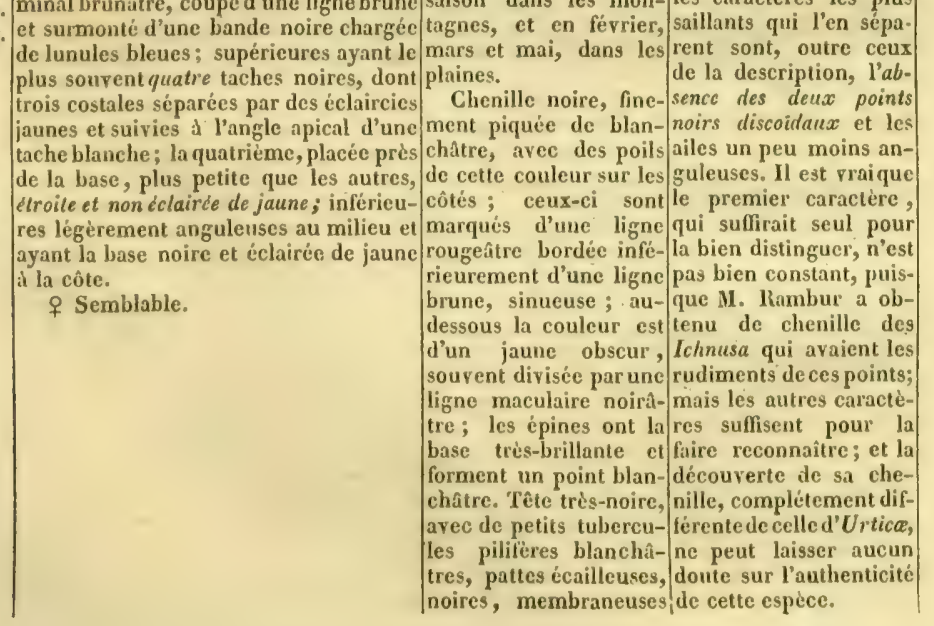


1 POLYCHLOROS. Lin. Fab. Ochs. Bdr.

Hub. $8 s-82$. Van. Polychlore. God. pl. 6. fig. 2. J.a grande Tortuc. Engr.

Pexctum arbon. Dahl.

Esp.

Testudo.

Pyrrhomelana. Hub. $845-846$.

XANTHOMELAS,

Envergure, 58 mill.-diles dentées,

Le fond est d'un fauve plus vif et lo point du dessous des inférieures est plus petit et très-arrondi.

Les deuxieme et troisieme taches noires costales sont réunies en une seule grande tache noire; il n'existe sur le disque que deux points noirs presque réunis et formant une espèce de bande parallèle au bord interne; les inférieures ont le fond entièrement d'un brun noir, avec une seule bande fauve séparée par les nervures. Dessous plus foncé et sans point blanc central.

Fond plus virement coloré et taches anté-terminales d'un bleu plus intense. Ochs. God. IIub. 85-86. de la forme de celles de Polychloros Volliynie, Crimé, Li-
Bdv. Icon. pl. 2 . La Tortue moyenne. nales et anté-terminales que chez Po-bre

Engr.

\section{Dans toute l'Furope,} point

\section{Autriche.}

verdâtres, avec une titclic noire. Vit en société sur l'Urtica hispida.

Chrysalide scmblable à celle d'Urlica.

Elle est très-commud jardins, routes, prome-ne. Chez beaucoup 等, nades plantées d'ormes, d'individus, la bande pées, avec une bordure jaunatre cou-etc., etc. En juillet, noire anté - terminale d'une bande noire assez étroite, un peu Chenille noire, à épi- est longée par une séric quée de croissants d'un bleu var- nes fauves, avec une li- de taches jaunes inceret surmontée aux mêmes ailes d'une foncé, diviśé en deux jamais chez la Xanthobande incertaine plus claire que le fond; par une raic noire et melas. On prend aussi et les discoidalesarrondies, au nombre pattes noires. Vit en discoïdale placéenu-desvarié de brun et de jaunatre, avec un juin et aout. Elle est elle vit en bonne intelChrysalide d'un brun des Cétoines, des Céun pen incarnat, avec rambycins, etc. etc. des épines dor'sales et Elle est facile à élever quelques taches métal- de chenille comme liques. $\quad$ toutes ses congénères.

Elle mérite à peine le nom de variété.

Cette variété est toutì-fait accidentelle. Engramelle en figure une (pl. LV, fig. 5. l. k.) qui s'en rapproche extrêmement.

Celte variété, que nous n'ayons pas vuc est rapportéc ici par II. 'Treitschke, qui pense qu'elle pourrait être une hybride de Polychloros et de $X$ anthomeIns:

On la distingueva toujours facilement de caracteres indiqués, par l'absence de la bande lychloros, mais la bande noire plus Chenille noire, arec jaunftre qui surmonte large, surtout aux inférieures, ou elle deux lignes dorsales la bande anté-terminan'est point lunulée et où clle est mar-blanches, épines noires le noire des inférieures quée de taches diun bleu plus violet; moins branchues que chez cette dernière. La supérieures avec des taches noires celles de Polychloros. Xanthomelas, quoique dont les costales plus grosses, surtout ( La rangée dorsale assez commune dans 
|les deux dernières, qqui sont séparées par|qu'on observe dans/certaines localités, n'est| une ćclaircic jaunâtre et suiviesả l'angle celle-ci manque dans pas extrêmement réapical d'une tache blanche. Taches du Xanthomelas.) Tête et pandue dans les collecdisque grosses, excepté l'externe, quilpattes écailleuses noi-tions. Nous ne savons est beaucoup plus petite et lunulée; infé- res, pattes membrancu- ce qui a déterminé linricures arec une grosse tache noire ses fauves. Elle vit en gramelle à l'appeler la costale très-légèrement éclairée de société en juin et juillel Tortue moyenne, dénojaunâtre.

sur les Salix, capraa, mination qui ferait of Plus grande et un peu moins an- vitellina et glauca. gruleuse.

Chrysalide brunatue, passage de la petite it la saupoudrée de bleu, grande 'ortue, tandis avec des épines dorsa- qu'elle est au contraire les, mais sans taches intermédiaire entre $\boldsymbol{P}_{0}$ métalliques.

lychleros et $V$. album.

V. Al.DUM.

Finb, Ochs, Gorl.

Hub: 85-84.

Dup. pl. 25. fig. 1-2. Bdr. Icon, pl. 2 i. fig. 1 Le $r$. lilanc. Ingr.
TIINGULUM.

Fab. Ochs.

I. Album. Bdr. Hub. 90-91.

God. pl. 10 3. fig. 1-2.

Fals.

F. ALвем.

C. Alistem, rar, Goul.

L. Album, var, Bdr.

I. \เ.แ1ห.
Envergure, 58 mill.-Ailes dentées, trìs-anguleuses, d'un fauve foncé, avec une bordure d'un jaune roussattre précédée d'une bande brune; supérieures avec de nombreuses taches noires, dont les costales plus grosses, séparées par assez semblable i celle V. Dans les exemplaires de légères éclaircies jaunatres et suivies de $C$. album; clle a une que nous possédons il d'angle apical d'une tache tres-blanclie; raic dorsale violette ressemble à un petit inférieures plus sombres, avec une tache ou noire, et deux lignes point à peine triangucostale noire dicisée par une tache trés-Intérales d'un jaune laire. Nous avons vu blanche et une série anté-terminale de paille ou fauve; les une variété dont le destachesjaunatres. Dessous d'un gris vio- épines sont blanches. sous des ailes est preslâtre plus loncé jusqu'au milieu; supé- Vit en juin sur l'orme que unicolore et en gérieures arec une serie ante-terminale et le saule.

de points bruns; inférieures avec une tache discoïdale d'un blane terne en lorme de $\mathbf{V}$.

ㅇ Plus grande.

Enyergure, 45 mill.-Ailes dentées et fortement anguleuses (les supéricures ayant deux angles principaux, les inféricures quatre), d'un jaune fauve, avec une ligne terminale brune, etroite, surmontée d'une autre ligne brune ou rousse également étroite et interrompue, précédée elle-même d'un rang de lunules jaunes peu marquécs; supérieures arec cing points noirs dont trois discoïdaux et deux près de la côte, et une petite bande noire etroile au bout de la cellule; inférieures aree une tache coslale noire, dont on n'apercoit bien qu'un point is la base et deux a l'extrémité, et un point dans la cellule; dessous d'un jaune-brunâtre tres-clair, strié de brun; les supéricures avec une ligne médiane delaye axant d'arriver is la côte, les inféricures avec un signe central en forme de $<$.

$q$ Un peu moins anguleuse, ì coulenr plus vive et plus teintic de roux en dessus.

Plus petite, taches discoidales des supérieures plus grosses et un petit trait noir apical; inférieures arec une seule tache noire et le hord postéricur olsscur. Dessous plus foncé et trait des inférieures ayant la forme d'un F.
Chrysalide d'unblanc autant de modifications jaunitre, arec quatre pour la couleur que grandes taches argen-celle de $C$. album. ties.

France méridionale,

Fille est commune. Ilongrie, etc., etc. Son corselet est, comDans les jardins, le me celui de la suivante, long des haies, etc. En garni de poils verts. juin et septembre. lille aime it se poser sur le trone des arbres cariés, sur. les murs qui séparent les jardins, les fruits pourris, etc. Quoirque son rol soit vif elle n'est pas farouche et se laisse facilement approcher. Nous l'avons prise abondamment all environs de ll ontpellier. Nous lui restituons le nom de Fabricius,quinous semble plus juste que celui de Godart et qui d'ailleurs lui est antérieur.

liussic méridionale.

Nous n'avons point vu cette rarićté, nous ne saurions done dire si elle appartient plutôt aे C. album qu' à triangulum.

C'est d'après l'autorité de MI. Bdr. que 
C. MLBLM. Liı. Fall. Ochs. Bdy. [1]ub. $9^{2-9 j}$. Vial. accid. $65 z-658$. Van. C. blanc. God, pl. 5. fig. 5 et 5 tert. fig. 1. Le Gamma. Eugr.
Envergure, 35 mill,- - Ailes dentées

et fortement anguleuses (angles comme dans Triangulum, mais plus fortement fardins, pres, chemins, spatulés ì leur extrénité), d'un fauve juillet et septembre. vif", avee une bordure anté-terminale d'un brun roux, surmontéc d'un ran Chenille à épine de taches d'un jaune faure, toujours faure roussate, d'un cerclées supúrieurement de roux aux tous les anneaux il pasinféreures; supérieures ayec des taches lir dn cinquième enticnoires très-marquées, dont une au bout rement blancs et maude la cellule large et rectangulaire; in-qués d'une bande lalérieures ayant outre la tache costale térale de la couleur du deux autres taches sur le disque. Des- fond; épines de la cousous jaune ou brun, varié de brun fon- leur des partics quileur cé; supérieures arec la moitié anté- donnent naissance; têrieure plus foncée et nettement coupée te noire, arec deux pemême d la côte; inféricures ayant au tites aigrettes de nême bout de la cellule un signe d'un blanc couleur. Vit solitaire brillant en forme de C., les quatre ayant :ur l'orme, Ulmus c.tmun rang anté-terminal de taches d'un pestriset quelquesauties vert foncé plus ou moins marquées. o Semblable.

\section{(91) Genre XIY. ARGYNNIS (ARgynne).}

(Fab. Ochs. Dalm. Bdv, - Les Nacrés. Latr. Gud. (G. Argynne). arbres.
Chrysalide d'un brun clair, avec quelques nuances plusfoncées, le dos tres-creux et marqué.de six taches argentées. nous plaçons ici celte rariété, que nous n'avons pu nous procurer.

Elle est très commune et a les mêmes habitudes que la précidente. Il est rare qu'on la rencoutre fanćc quoiqu'elle vole beaucoup et rapidement. Elle varie assez, principalement pour l'intensité et la grandeur des taches noires. M. Marchand a obtenu de chenille la variẻté figuréc dans Iubner. (65j-658). 
Chartotta. Sowerby. Brit. Misc. tab. 2.

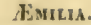

Acerby. Voy, au CapNorit.

ADIPPF. Lin. Fab. Ochs. Bdr. IIub. 65-64. God. pl. 5. fig. 2, et 5 sec. fig. 2. L.e grand Nacré. Engr.

Creonoxa.

Esp. Herbst. Adippe. var. God. Bdy. Ochs.

II ub. $859-860$.
Envergure, 58 mill. - Ailes un peu dentées, d'un fauve très-vif, avec de nombreuses taches noires et la frange fauve. Supéricures avec les penultieme et antépénultieme nervures très-renfles au milieu; inférieures avec une rangée discoidale de quatre à cinq points noirs,
dont l'anterieur beaucoup plus pelit que le suirant; dessous des inféricures d'un
jaune faure pâle, avecbeaucoup de taches argentées, dont quelques-unes ombrées
de ro: $x$, et un rang de points argentés cerclés de ferrugineux.

o D'un faure plus pâle, arec les nervures des ailes supéricures non renales. Lin. Fab. Ochs, Bdv. Hub, 61-62. God. pl. 2 G. fig. 5-5. Le Chiffire. Engr.
Elle ne differe d'A dippe qu'en ce que les taches argentées du dessous des ailes disparaissent complétement. et sont remplacées par des taches d'un jaune clair, excepte la prunelle des ycux ferrugineux, où la couleur argentéc persiste ordinairement.

Un peu plus grande qu' 1 dippe, d'un faure plus jaune, avec la frange blanchâtre. Points noirs un peu plus petits, dessous des inférieures d'un ton un peu verditite, ayant a peine quelques traces de ferrugincux et point de taches nacrées.

Envergure, 50 mill. - Ailes un pen lentés foures, ayec la base ct le bord de l'est et lu midi de la rare. Son rol ést rapide, iuterne trés-obscurs et de nombreuses France, Suède, Suisse, et elle se pose peú. On taches noires. Supérieures avec les ner- Russie, Iollande, etc. la distinguera d'Adippe rures du disque et du bord interne lé-En juillet et aoat. parsataille, lesnervures gerement renfées. Dessous des inférieu- Chenille brunatre, des premières aileschez res d'un jaune pâle un peu cerdâtre, sur. arec une ligne dorsale le mâle et les caractères tout $d$ lu cóte et au bord interne, avec les noire bordée de blanc, indiqués en italique.

Mêmes localitis.

Elle n'est point rare dans nos environs, où elle vole avec les $A d i p-$ pe ordinaires. En Suisse, on ne la trouve jamais en-deçà des $\Delta$ lpes qui séparent ce pays du Piémont, tandis qu'elle est commune alt-deli.

Nous ne sommes pas bien sars que les indiviclus sur lesquels nous frisons cette description soient de véritables Chlorodippe. Ils ont été pris en Sicile, au sommet des monts Madonicrs, par M. Alex. Lefebre * Forêts montagneuses Elle n'est pas très-
Elle a été prise cn juillet dans le Bedfordshire. M. Lefebvre, qui I'a vue dans la collection d'llaworth, assure que ce n'est qu'une variété.

Oschs. la rapportc d̀ l'A glaia, et Dalmani qui a vu l'exemplaire original, assurc que ce n'est qu'une varièté accidentelle.

Dans unc grande On la trouve commupartie de l'Eurape, fo- nément dans les bois ts. En juillet.

d'une certaine étendue; Chenille d'un brun elle affectionne spéciaune bande dorsale ronce. Elle offre un blanche, interrompue, nombre prodigieux de des stries obliques variétés. Plusicurs aupattes membraneuses l'Adippe de Linné se l'un brun plus clair. rapportait di la Niobe, Tete noirâtre. Vit en par la ruison, disaientjuin sur les $V$ iola odora-ils, que la dernière se trouve seule en Suede. avec des taches et la deux espèces $y$ sont base des épines dorsales communes. argentíes. (n) 
Waln.

AGLAOPE. cyclopédie.

Enis.

Schön.

fnervures noires; beaucoup de taches, fune ligne latérale noiquelquefois arźntées, bordées de noir, re et, entre les deux, et une rangée d'yeux ferrugineux à pru-des taches triangulaires nelle argentée.

blanches. Tête et pattes

f Plus grande, plus pâle, d'une d'un roux juunatre, teinte un peu verdatre; et à ailes supé- épines d'un blanc sale. rieures plus arrondies.

Vit"en mai sur la Violette, Viola odorata.

Chrysalide d'un gris brun, arec quelques ta-

ches argentees.

Dessus des quatre ailes plus clair a la
Alpes.

base et taches marginales du dessous

des secondes ailes plus fortement bordées de rougeâtre.

Elle differe de Niobe par sa couleur fauve un peu plus terne, par la base plus largement noirâtre, les taches noires plus prononcées et le sommet de l'aile jaunâtre à la côte. En dessous le le disque des supérieures est d'un fauve un peu rosé, et celui des inférieures ne differe de la variété sans nacre de Niobe qu'en ce que les taches ferrugincuses y sont très-nombreuses et très-foncées.

CYRENE.

Bon. II ub. $822-825$. Bdr. Icon. pl. 21. fig. 1-3. pl. 18. fig. 3-í.

LATIIONIA. Lii. Fab. Ochs. Bdr. God, pl. 3. fig. 5. Lathona. Ilub. $59-60$. Le petit Nacrd. Engr.

Asp
Envergure, 5o mill.-Ailes un peu dentées, arecla base un peu plus obscu- Corse et de la" Sardaire et des taches noires, rares, petites et gne. En juillet. isolées; supérieures un peu arrondies au . bord marginal. Dessous des inférieures d'un jaune pâle, un peu verdâtre à la basc et al la cóte, arec beaucoup de taches argentées, petites, et dont les anté-marginales presque réniformes et surmontées d'un épais triangle d'un brun roussatre, et un rang d'yeux inégaux de la même couleur, pupillés d'argent.

o D'un fauve plus terne et plus sombre en dessus, avec les taches noires un peu plus grandes.

Envergure, 36 mill.-Ailes légère- Dans toute l'Europe, ment dentées, les supérieures ayant al bois, prés, jardins-, l'angle apical une partie saillante arron- chemins verts, etc., die, les inférieures formant un coude etc. En mai, aoot et vers le milieu du bord marginal; les qua- septembre.

tre d'un fauve un peu terne, avec lit ba- Chenille d'un gris se.et le bord interne largement reritatres ligne aime à se poser ì terre. dies. Dessous des inférieures d'un faure deux liznes latérale, très-clair, avec beaucoup de taches ax-d'un jaune brunâtre et gentées, dont cinq trés-larges sur le dis- les incisions de la même que, et un rang anté-marginal d'autres couleur. Pattes et. épiégalement assez larges et surmontées nes d'un jaune d'ocrc. d'une bande ferrugineuse marquée Vit en mai et juillet sur d'yeux à prunelle argentée.

ㅇ Plus grande et semblable.

la Viola tricolor, l'II $\ell-$
dys. onobrychis et l'An-
chusa offecinalis.
Chrysalide d'un gris
bleuatre, avec le dos
très-creux et une large
tache blanche sur cha-
que côté de l'abdomen.

Les taches nacrées du dessous des in-
Nous n'arons pas ru cetle variété, que nous décrivons d'après Godart.

Euyoyéc sous ce nom à M. Marchand par M. Schönherr.

La coupe d'ailes et la petitesse des taches noires distinguent cette Argynne de toutes les précédentes. M. Rambur, quil'a prise abondamnient en Corse, en a probablement élerí la chenille, mais ilne l'a pas encore publiée. Le papillon est encore peu répandu dans les collections, d cause des localités circonscrites qu'il habite.

Elle cst très commune et paraît pendant une grande partie de l'année, mais clle est plus abondante a Cettc variété, que 
Lathona. Hub. 613? férieures sont réunies en forme de bandes, et les taches noires du dessous des supérieures sont plus grosses et absorbent en partic la couleuridu fond.
PAPHIA. Hub. 69-7o.

God. pl. 3. fig. 1. et 5 sec. fig. 1 .

Le Tabac d'Espagne. Engr.

VALESINA.

\section{Esp. Herbst.}

Paphia.var.Hub. 6 ; -68. riété 9 .

Le $V$ alaisien. Engr.
Lin. Fab. Ochs. Bdv.

Bdy. God. Ochs. va-
Euvergure, 75 mill.-_liles supérieures arec une partic saillante et arrondie à l'angle apical; inférieures dentées;

Dans toute l'Europe, ois. En juillet.

Chenille d'un roux nous n'avons point vue en nalure, nous semble tout-í-fuit accidentelle, elle se rapproche beaucoup de la fig. 615 d'llubner.

Elle est trés-commune dans les grands bois, ou elle se pose volonliers sur les ronces et anté-terminal de taches quadrangulai- sur les côtés, avec une les chardons en fleurs. anté-terminal de taches quadrangulai- sur les cótes, avec une les chardons cn heurs. taches arrondies noires; supérieures parée par un filet brun. élevé. Rösel, qui repréayant en outre les quatre dernieres ner- Tête et pattes brunes, sente très-lien la fevures tres-renflées, noires et velucs. Des- épines d'un jaune fauve melle (tab. VII), n'a sous des inférieures d'un jaune clair, à sommet brun, celles point indiqué sa teinte luisani, glacé de vert sur le disque, et du cou beaucoup plus verdatre. Aurait-il roude blanc violâtre nacrí au bord mar-longues.

lu figurer une variété ginal, avec plusicurs bandes nacrées et On la trouve à la fin oppesée à Valezina, ou un double rang antć-marginal de gros de mai dans les en-bien est-ce, ce qui est points verts.

droits humides des bois, plus probable, une ero $\mathbf{A}$ ailes plus arrondies, d'un fauve sur la $V$ iola canina, et reur d'enluminure? mélangé de vert en dessus, avec les quelquefois, dit-on, sur Cette Argynne varie taches noires plus larges, sans renfle-d'autres plantes de fa- peu; cependant Engraments aux nervures; et ayant le fond des milles éloignées. melle en donne des vainiéricures en dessous d'un vert plus Chrysalide d'un gris riétés accidentelles asfoncé que le mâle. violatre strié de brun, sez curieuses. Pour avec quelques taches nous, nous ne connaisargentécs sur le dos et sons qu'une variété près de la tête, et le constante c'est celle que bout de l'abdomen ar- nous décrivons. ci-desmé de deux grosses sous. On observe de pointes.

Difrèro de Paphia par une teinte gétemps en temps aussi des individus hermaphrodites dans cette espèce; M. Eschier, de Zurich, en possède un lont les deux ailes de gauche sont, celles de Pap hia inale et les deux de droites celles de $\mathbf{V a}$ lesinu femelle. nérale d'un noir un peu verdâtre et par cantons méridionaux temps en temps cette deux taches blanchâtres placées vers de la Suisse, surtout belle variété dans la foles $\frac{3}{3}$ do la côte des superieures; le des- da ces tacheg et celui des inféricures est d'un vert plus foncé que chez la femelle de Paphia.

PANDORA.

Envergure, 80 mill.-Ailes supérieuOuest et sud de la M. Duponchel:l'a prise en notre présence. Nous l'avons prise nous-mêmes dans la forêt de Mormûle, accoupléc avec Paphia. M. Lel'ebvre nous, assure avoir ru le: mâle de cette variété.

Ochs. Bdv. II ub. 21-72. 606-607.

res avec une partie saillante et arron作

Cynara. Fab. God. pl. les quatre drun faurc entirement glace Hongric, Autriche, ete. 7 c. fig. $1-2$.

Le Cardinal. Engr. decert excepté à la côte et a l'angle api- En juin et juillet.

cal des supérieures, avec des taches

noires dont une double séric de points surmontés aux inféricures d'une bande en zig-zag: supéricures avec les deuxièmes et troisiemes nervures, a partir du| ou elle est trés-commune. Elle aime à se poser sur des chardons en lleur, et vole assez rapidement si on l'effarouche. 
bord interne, très-renflées et velues. 1)essous des supérieures d'un bea' rouge, avec le sommet verdâtre et des taches noires. Dessous des inferieures vert, ayec trois bandes argentées ou jaunatres et une série antí-terminale de petits points argentés, ombrés de roussâtre.

잉 arrondic, ayant les taches noires plus grosses et les nervuresinon renflées.

LAODICF.

Fab. Ochs. God.

Dup. Suppt. pl. 8. fig. 1-2.

Bdr. Icon. pl. 21, fig. 4-6.
Envergure, 6o mill.-Ailes un neu dentées, les supérieures un peu sinuées mée, Valachie, Livonie,
au bord terminal, les quatre d'un beaulet., etc. En juin laupe, avec des taches noires, arrondies juillet.

pour la plupart. Supéricures avec les deux nervures du bord interne renflées et noirâtres: Dessous des inférieures ayant la premiere moitié d'un jaune verdatre, avec deux lignes d'un rouge brun et la seconde d'un pourpre violätre, avec deux séries ante-terminales de taches arrondies plus foncées. Ces deux parties séparées par une bande sinuée, irrégulière, maculaire et un peu argentée.

\& Plus grande, plus arrondic, avec les nervures non renflées et une petite tache blanche triangulaire à l'angle apical.

DAPHNE.

Fab, 0 chs. Bdr.

Ilub. 45-46.

fod. pl. 8 n. fig. $1-2$. La grande Violette. Engr.

Ochs. Bdv

INก.

God. pl. 8. fig. 3-4.

byctinna. Hub. 40-4n. dies, dentées, d'un beau fauve rif, arec
Envergure, 55 mill. - Ailes arronde l'est de la France, des infes Allemarne, environs $\mathrm{d}^{\prime} \boldsymbol{A} \mathbf{x}$ et de des inferietures ayant la première moi- Honoric, Russic, etc. Schélestadt, sur les liú d'un janne clair, avec deux lignes Fin juin et courant de médianes rousses, dant l'intéricure um-juillet. brée supéricurement de roux an brun ainsi que celui de la de la base, et la seconde moitié variée noir, aree des lignes suivante, s'éloigne de de rose, de jaune, de roux et de violet, longitudinales jaunes celui de ses congénéres fondus ensemble et chatoyants, avec ou blanchitres, et une et se rapproche des une série d'yeux ferrugineux mélés de large bande dorsale de mélitées. noir, à prunelle verdâtre.

o Plus grande, plus pâle, et ayant a six rangs d'épines les taches plus grosses. dies, dentées, d'un fanve clair, avec la ct dos taches noires; pt est de la France, dans celle de la précédente, celles de la série anté-terminale lunu-les forêts. En juin et est le principal caraclées' et les précédentes punctiformes et juillet. assez petites. Dessous des inférieures Chenille d'un jaune Ia bande médiane ayant la première moitié comme cliez blanchâtre, striée lon-violâtre du dessous des Daphne, mitis avec les partics rousses gitudinalement debrun, inférieures, est quelplus foncées et plus anếices, et la se-avec une ligne dorsale quefois à peine visible. conde d'un fauvetrès-panaché du même brune et des épines de Au reste, celle bande roux, avec une bande médiane incer-la couleur du fond et qu'ou commence à taine et quelquefois le bord marginal ciliées de noir. Tête mi- apercevoir danscetteesinférieur d'un blane violatre, et une sé-partic de brun et de péce', se retrouvera plus rie d'yeux roux it prunelle fauve.

o Semblable, mais plus grande. jaune pâle. V'it en mai ou moins distincte dans surl'ortic(Urticaurens). presque toutes les Ar- 
THORE.

Ouhs. Goul. Bdy.

Ilub. $57 \imath-573$.

Dup. Suppl. pl, 19. fig. 1-2.

Bdv. Icon, pl. 20. 6ir. 5-4.
Envergure, 42 mill. - Ailes arrondies, ì peine denticulées, faures, saupoudrées de noirâtre par places, avec des taches noircs très-largesqui absorbent presque la couleur du fond, surtout aux inférieures. Dessous de celles-ci d'un roux ferrugineux, avec la base et une bande transverse d'un jaune d'ocre, derrière laquelle est une bande interrompue d'un blanc violatre, un peu nacré, puis une séric de gros points ferrugineux inégaux, non pupillés, puis enfin une hande presique terminale d'un gris bleutetre on violatre.

\& Un pen plus grande.
AMATHUSIA.

Fab. Ochs. Bdy. God, pl. 8 I. fig. 5-6. Titania. Ilub. 47-48. Viana. 51 \& 5 ?.
TREYA.

God. Hub. 55-56.

Dup. Suppl. pl. 19 fig. 5-4.

Freya. Oc ]: Bdr. Icon. pl. 19. fig. 4-5.
Envergure, 44 mill. - Ailes dentées, d'un fauve foncé avec la base, et des ta- Tyrot, ches noires, dont celles de la rangéc an- de la France, etc., etc. té-terminale trés-sagittées en dessus et En juillet et aoat. en dessous. Dessous des inféricures Chenille d'un gris d'un rouge briquete, avec de petites ta-foncé, arec une bande ches d'un jaune clair id la base, unc dorsale maculaire noire bande transverse du même jaune, lise- et des épines jaunes,
rée de noir et envalic en partic par la dont labase est entourée couleur du fond, puis une bande d'un de noir. Tête et pattes blanc-violattre nacré, puis une série de noirattres. Se trouve it gros points d'un ferrugineux foncé, la fin de mai sur le $P_{0}-$ presque igaux, appuyes sur la pointe des lygonum bistortu. taches sagittées. Antennes d'un gris- Chrysalide d'un grisjaunâtre, annelées de noir. dic.

$q$ Un peu plus grande et plus arronbrun, avec les stigmates

Envergure, 40 mill. - Ailes d'un Laponie, Suède, Isfauve pâle et terne, avec la base large- lande. En juillet.

ment noiratre, surtout aux inférieures, et des taches noires, dont celles de la rangée anté-terminale chevronnées et surmontées d'un rang de taches rondes, dont les intermédiaires ordinairement très-grosses. Dessous des inférieures roux, arec quelques taches d'un blanc jaunâtre à la base, une bande transferse du même blanc saupoudrée de roux dans son milieu et fortement bordée de noir, puis une autre bande plus étroite, sinué, du meme blanr, et un rang terminal de petites taches étroites, encore lu même blanc, surmontées de chevrons noirs et de taches également noires, petites, isolées et d'inégale gran-l verdâtre, nuancée de bordés de blane et des épines assez longues à base argentée.

Chrysalide d'un brun|gynnes suivantes. L'Ino jaunatre, aveclestuber- est commune dans la cules épincux d'un jau- forêt de Mormâle, où nousl'arons prise abondamment. Elle se pose de préférence sur les ronces.

Elle varie pour l'intensité des taches nojres, mais elle est toujours plus sombre que les autres espèces. Elle était autrefois fort rare, mais elle s'est répandue dans les collections depuis qu'on l'a trouvée, non pas, comme le dit

M. Duponchel, dans une grande partie de la Suisse, mais dons deux cantons seulement (Berne et Unterwald), dont elle n'habite que des localités très-restreintes, comme la vallée du Hasli, les pâturages de Reusti et quelques parties des Alpes surèncs.

C'est principalement de Suisse qu'on reçoit cette espèce, qui y est très-commune. Elle se tient ordinairement dans les vallées et recherche les prairies humides et ombragées.

$$
\text { ats }
$$

Outre les différences qui ressortent de cette description, on distinguera cette espece d'Amathusia par sa taille, ses couleurs généralement plus pales et d'un autre ton, par ses antennes, etc., etc.M.Bdr. la compare d Selene, mais les individus que nous possédons et ceux que nous arons rusn'ont que des rapports bien éloignés avec cette Arm gynne. Du reste, la Freya varic pour la laille et peut-être aussi 


\section{G. ARGYNNIS.}

\begin{tabular}{|c|c|c|c|}
\hline & $\left\{\begin{array}{l}\text { deur. Antennes rousses, légèrement an- } \\
\text { nelées de noir en dessns. } \\
\text { o Un peu plus grande ct plus arron- } \\
\text { die. }\end{array}\right.$ & & $\begin{array}{l}\text { pour les couleurs. Elle } \\
\text { est rare dans les collec- } \\
\text { tions. }\end{array}$ \\
\hline $\begin{array}{l}\text { Var. A. Nonts. } \\
\text { Freijn. Hub. } 71-772 \text { ? } \\
\qquad\end{array}$ & 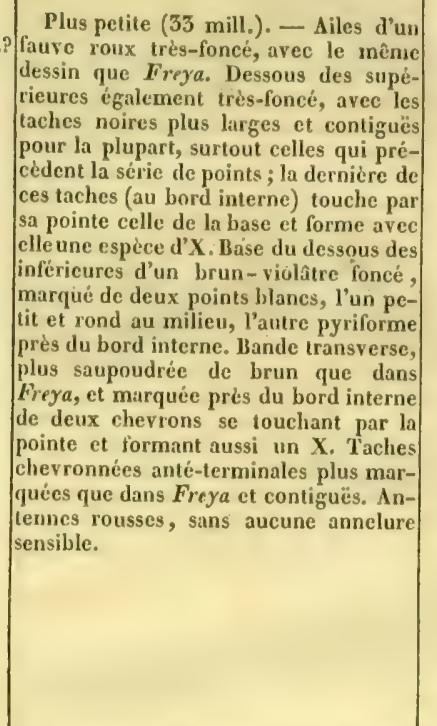 & res & 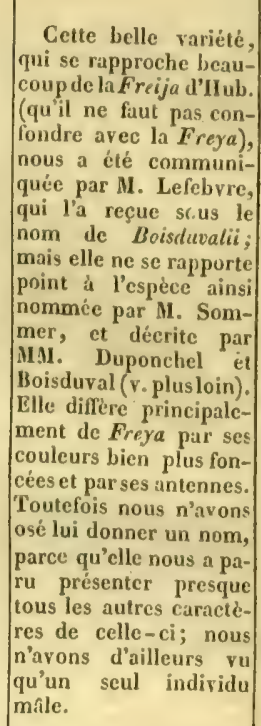 \\
\hline $\begin{array}{l}\text { POLARIS. } \\
\text { Bdr. Index. Icon. pl. } \\
\text { 20. fig. 1-2, } \\
\text { Dup. Suppl. pl. 20. fig. } \\
\text { 1-3. }\end{array}$ & $\begin{array}{l}\text { Envergure, } 58 \text { mill. - Ailes d'un } \\
\text { fauve terne, avec la base largement } \\
\text { noirâtre, surtout aux inférieures, et des } \\
\text { taches noires dont celles de la série an- } \\
\text { té-marginale non chevronnées, isolées ou } \\
\text { réunies. Dessous des supérieures arec } \\
\text { des taches noires étroites et une série de } \\
\text { traits blanchátres touchant par une ex- } \\
\text { trémité le bord marginal. Dessous des } \\
\text { inférieures d'un brun-riolître foncé, } \\
\text { avec beatucoup de taches blanches, celles } \\
\text { du milieu formant une bande transverse } \\
\text { saupoudrée de brun au milieu, les sui- } \\
\text { vantes une autre bande peu arrêtée et } \\
\text { appuyée sur des points noirs, et les ter- } \\
\text { minales carríes ou en forme de T et en- } \\
\text { tourées de brun. Espace qui est entre el- } \\
\text { les et les points noirs un peu plus clair } \\
\text { et plus jaunatre que le fond. } \\
\text { \& Semblable. }\end{array}$ & Laponie, cap Nord. & $\begin{array}{l}\text { Elle est jusqu'ici ex- } \\
\text { trêmementrare et rarie } \\
\text { un peu pour la taille. } \\
\text { L'individu figuré par } \\
\text { M. Duponchel sous le } \\
\text { n² est une variét仑 re- } \\
\text { marquable si les cou- } \\
\text { leurs n'en sont pas ou- } \\
\text { trées. La massue des } \\
\text { antennes est très-forte } \\
\text { chez cette espèce, et la } \\
\text { tige en est à peine an- } \\
\text { nelie. }\end{array}$ \\
\hline $\begin{array}{l}\text { FRIGGA. } \\
\text { Ochs. God. IIul., 49- } \\
\text { 5o. } \\
\text { Dup. Suppl. pl. 19. fig. } \\
\text { 3-5. } \\
\text { Bdv. Icon. pl. 19. fig. } \\
\text { 6-7. }\end{array}$ & 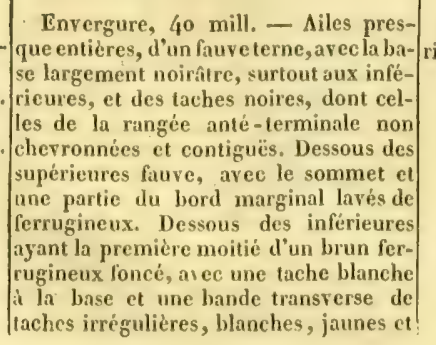 & $\begin{array}{l}\text { Suède, Laponie mi- } \\
\text { ridionale. En juillet. }\end{array}$ & $\begin{array}{l}\text { Elle est très-raredans } \\
\text { lcs collections, comme } \\
\text { la plupart des espèces } \\
\text { boréales. }\end{array}$ \\
\hline
\end{tabular}


|saupoudrées pour la plupart de ferrugineux, mais non celte de la côte, qui cst blanche et souvent isule. Seconde moitié des mêmes ầles $d^{\prime}$ un brun-violatre clair, éclairée de blanc intérieurement dans sa partic supérieure et traversée par une séric de points obscurs un peu ocellès et une rangée anté-terminale de lunules d'un violet obseur: le tout peu marqué.

o Plus grande et plus obscure en dessus.

DIA.

Iin. Fab. Ochs. Bdr. Hub. 51-35.

God. pl. I scc. fig. 1. c 4 quint. fig. 1.

In petite Violeltc. Engr.

CHARICLEA Hub. $768-769$. Dalman.

Borsulyalis. Som. Bdv. Icon. pl. 20. fig. 5-6.

Duip. pl. 20. fig. /.
Envergure, 54 mill. - Ailes légère-

Dans une grande pararent denties, lauves, avec la base i tie de l'Europe. Dan peine noirittre el des taches noires assez les bois un peu sees. grosses, dont celles du disque et de la En mai et noat.

base ordinairement assez rapprochées.

Chenille noire ou Dessonsdes supéricures faure, avecl'an-d'un brun foncé, avec

gle apical marqué de ferrugineux, le dos plus clair et maréclairé intérieurement de blanc-violatre qué d'une ligne longitunacré. Dessous des inféricures (qui sont dinale noire. Epines coupées tris-carrement d li cóte) d'un d'un gris blanchâtre, ferrugineux violatre, varié de jaune, pattes noire. Vit en avee une bande transverse de taches al- juillet et septembre sur lernativement d'un blane nacré et d'un différentes espèces de jaune à peine marqué d'atomes ferrugi- Viola.

neux, puis une bande bien continue et incertaine, d'un blanc-violatre brillant, et an rang terminal de petites taches blanches ou jaunes, très-isolées et surmontées d'une série de gros points d'un brun rouge, dont les intermédiaires pupillés de jaunc. Antennes n'étant pas sensiblement annelies.

\section{o Semblable.}

Envergure, 58 mill.-Ailes d'un assez beau faure jaunatre, arec la base noirître et des taches noires, dont les anté-terminales isolées et presque punctiformes. Dessous des supérieures fauve, avec le sommet et le burdmarginal trìs-marqués de brun rouge et la base et le disque de signes noirs. Dessous des inféricures d'un rouge brun, plus foncé à la base, qui est marquée de petites taches nacrées, puis traversé par une bande bien distincte d'un blanc nacré mû́lé de taches jaunâtres et un peu saupoudrées de roux, bordée extéricurement de noir. Reste de l'aile un peu mêlé de jaune, avec une bande incertaine et peu sensible d'un blane violatre, une série de points ferrugineux, dont quelques-uns légèrement pupillés et un rang marginal de taches d'un blane nacré, ovales - oblongucs, bien marquées et surmontées de iriangles ferrugincux.

\section{\& Semblable.}

Sous ce nom on nous a communiqué trois Argynnes différentes. La première est celle dont nous parlons a l'article Freya; la deuxième est la Chariclea, qui a été envoyée sous ce dernier nom et dans laquelle quelques personnesveu-
Elle n'est pas rare. Elle plane en volant et ae s'éleve jamais beaucoup all-dessus du sol. Ses ailes inférieures se rapprochent déjì un peu par la coupe de celles de la Pales. 
1 MALES.

Fab. Ochs. Bdr. II ub, 34-55. God. pl. 9 x. fig. 12 (Type).

La Pales. Engr.
Yar. accid. 61 $z-618$. tent voir une simple variété de Boisdurabeaucoup de l'Arsilache Hubner, et nous semble n'en être qu'une modification. Pour nous, nous pensons que l'argynne Boisduralii n'est autre que la Chariclea d'Ifubner et de Dalman, qui varic beaucoup at ce qu'il paraît, et qui cst si peu connue et si rare qu'clle a bien pu donner lieu à ce double emploi. lii; la troisieme enfin se rapproche

$$
\text { rxuchisur }
$$

fomme touchant toutdi-fait la question. Nous avons vu trop peu d'individus de chaque espèce pour nous prononcer décidément ì ce sujet; nous appelons sculement sur ce point l'attention des entomologistes que leur position met à même de le vérifier.

Envergure, 32 mill.-Ailes d'un beau Alpes, Pyrénées, Elle est très-comfaure vif, avec la base et des taches Suisse, Itolie, Norwège, mune et varic extrêmenoires ordinairement petites et isolées, Suède, Laponie, etc. ment. Les femelles surles supérieures un peu aiguës au som-
met et ayant dans la cellule et près de Les auteurs alle-- $\begin{aligned} & \text { Eariétés entre elles. Le } \\ & \text { tout affent une foule de }\end{aligned}$ la base deux points noirs superposés; les mands rapportent a coudedes ailes inféricuinférieures légèrement polygonées, cou- cette espèce la chenil- res la fera toujours dispées droit au bord d'en haut et for-le figurée a tort par tinguer de toutés celles mant a la cinquieme nervure un angle Ifubner comme étant du genre; mais nous ou conde très-sensible; dessnus des su-celle de Sclene. Celtedevons dirc que cher périeures ordinairenent peu ou point opinion, comme le dit quelques individus ce taché de noir; dessous des infériemres Mi. Treitschke, acquiert conde est moins distinct. varié de rouge briqueté et de jaune tine graude probabilite Elle habite les prairies clair, saupoudré de brunâtre près dusi l'on considère que élevées des montagnes, corpy, ayant al la base un point blanc toutes celles des espé- aime à se poser sur les sur une bande ronge et des taches ar- ces voisines sont con- fleurs et se laisse prengentées, dont les plus apparentes au nues. Voici sa descrip-drefacilement. Dalman bout de la cellule, ì la cóte et au bord tion : prétend que lar variété terminal; ces dernières surmontées de Elle est d'un noir de $61 ;-618$ Hub., doit se de chevrons et d'une série de points velours, arec une ligne rapporter it Selene. ferrugineux, dont celui du cinquieme sur le dos et deux sur Nuus ne sommes pas de espace internervural souvent nul, ou les côtés plus obscures cet avis, car elle offre seulement, visible par transparence dn que le fond; les épines bien tous les caractères dessus; ce cinquième espace étant lui- latérales manquent sur de Pulcs. AI. Bugnion même occupé pạ une large tacheles premier, deuxié- nous signale, sons le jaune.

me, troisième et on-nom de Palemelas, une zième anneaux; cellẹs variété qui s'en rapprodu dos sont placées sur che assez et qui nous des taches d'un jaune semble tout-i-faitaceicitron et sont elles-mê- dentelle.

mes de cette couleur jusqu'a - leur milieu. La têt sont d'un brun roussittre Elle vit en mai sur la Viola Montana.

Isis.

Plus grande, d'un fauve plus vif et Hub. 38-39. 563-564. plustougeâtre; le dessous des inféricures Pales. var. God. Tr. est, moins chargé, de rouge et la séric Bdr, etc.

AnsILACHE. IIub, 36-37. Treits. Suppl. p. 12. Schneid. Freyer. Pales. var. God. Bdr. Napar. Dup. Suppl. pl. 48. fjg. 5-6.

tamment taché de noir. Le dessous
Mêmes localités. de points anté-terminaux y est ì peine distincte.

Un, peu plus grande que Pales. Lilçs plus larges et bien plus arrondies; supéde des insues au sommet, cou-Constance. Dans les sis, et derra probablede des inféricures peu sensible; taches prairies sylvatiques et ment constituer unc noires plus, grosses, surtout celles du dans les plaines basses. espèce distincte, surdisque Dessous des supérieures cons- En juin. des inlérieures est semblable jusqu'i la bande transverse; mais celle-ci est plus régulière, moins coudéc au milieu, le reste de l'aile est plus saupoudré de rouge; la bande, d'un blane vio- 


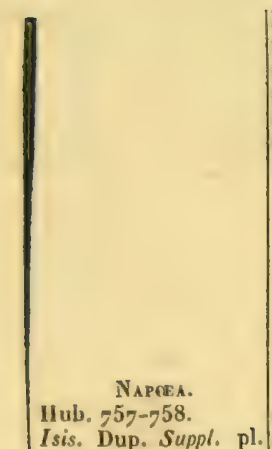

latre, y est bien plus distincte; les points forment une série bien continuc, sont souvent pupillés, et la tache jaune du cinquième espace internervural est toujours moins nette, plus rétrécic et très-souvent tout-â-fait nulle.

Isis. Dup. Suppl. pl 48. $\mathrm{fig} .7-8$.

Cinariclea. Ochs.? God. Encycl.

Dup. Suppl. pl. 48. fig. $7-8$.

EUPHIROSYNE.

Lin. Fab. Ochs. buts. llub. 28 i 3 o.

fiod. pl. 4. fig.

4 tert. fig. 2.

lées. Dessous des inférieures d'un jaune citron carié de rouge vif, arec une

Envergure, qo mill: - Ailes entie- Dans toute l'Lurope, res, arrondies, d'un beau fauve, avec la bois. Ĺn mai, juillet et base et des taches noires, dont les aont. Chenille noire, ayec vidus pris en Laponie une ligne laterale de sont souvent plus obsbande discoildale de la première con- petites lignes blanches ques variétés, entrauleur, au milleu de laquelle est une ta-qui tendent a se rappro- tres une appelée Finche argentée, et sept taches terminales cher sur le dos de cha-gat par Laspeyres et également argentées et surmontées de que anneau. Pattes citée par Dalman, qui chevrons et de points rouges.

o Plus grande, plus arrondic et ayant tres; épines noires, sine. ordinairement la base plus largement quelquefois jaunes sur noiratre.

de connaitre les chenilles. Nous avons vu plusicur mâles, mais une seule femelle, qui nous a paru n'en différerque par les caractìres ordinaires. Nous ignorons quels sont les motils qui ont déterminé ii. Duponchel at transporter a celte Argynne, le noin de Napcea et it celte dernière celui d'I sis.

Elle se trouve avec Pales, mais plus rarement qu'clle.

Nous n'avons. vu qu'une femelle de cette Artzyne, que nous ne saurions considérer que comme une des innombrables variétés de Pales; le dessous participe un peu d'Amathusia, mais on y retroure tous les caractères de Pales. Le bord terminal en dessus est continu dans l'individu que nous avons vu; mais Ochsenheimer dit qu'il est ordinairement entrecoupé de jaune, ce qui s'observe chez les femelles de Pales. Cette Argynne n'a rien de che arec notre Chariclea, bien qu'elle ait été envoyée en même temps et sous le même nom à M. Lefebvre.

Elle est très-communeetvaric peu, dumoins dans nos pays. Les indi-
Un peu plus grande que Pales. Desio brun a reflet violitre; dessous des marque de ronge sale ou brunatre,

o Semblablc. pace jaune comme dans Pales. le dos et à sommité noire. Tête noire. Vit en juin et septembresurles $V$ io Ia Canina et Montana. 
(1) SELENE. Fab. Ochs. Bdr. IIub. 26-27. Var. accid. $752-735$. 83. God. pl. 4 tert. fig. 4 . Le petit Collier al"gente Engr.

Tinera.

Ilub. $57-58$.
OSSIANUS.

Dup. Suppl. pl. 20. fig. 5.6.

Bdr. Icon. pl. 19. fig $1-5$.

Aphirape. IIub. 734735 . et Tricla (Exot. Samml.).

Ochs, Bds.

APIIRAPE,

Ilub. $23-25$.
Envergure, 38 mill. - Siles presque entières, d'un faure un peu terne, arec la base un peu noirâtre et des taches noires, dont les anté-terminales contiguës. Dessous des inférieures $d^{\prime} u n$ de gris obscur, avec les fectionne surtout les jaune d'ocre clair, varie de ferrugineux, épines d'un jaune roux grands bois. Cette Aravec une bande discoídale de la pre- ciliées de noir et deux gynne varie beaucoup, mière couleur marquée de trois taches taches blanches latéra- et se trouve également nacrées, puis une bande également na- les sur le premier an-en Laponie. En Suisse crée interrompue au milieu, et une sé-neau; tête noire, pattes clle vole sur les Alpes rie de sept taches terminales aussi na-d'un brun rouge: Vit en et n'habite pas la plaicrées et surmontées de chevrons et de arril et septembre, sur ne. points noirs.

o Semblable, mais ordinairement un peu plus terne.

La partic marginale est beaucoup plus pâle, et coupée par de larges taches noires; le dessous des inférieures est jaune jusqu'au milieu, et Ic reste est coupé par des taches rayonnées alternativement faures et argentées et dont l'intermédiaire s'avance plus loin que les autres.

Dans une grande par- Elle devance un peu ie de l'Europe, bois. Ia précédente, et est giéEn mai, juillet la violette.

Chrysalide d'un brun jaunatre, avee des taches et des épines noires.

C'est d'après l'autorité d'Oclisenheimer, que nous rapportons it la Sclene cctte rariété que nous n'arons pas vue en nature et qui nous semblerait plutî
Chrysalide obtuse, d'un gris brun, arec plusicurs points argentés sur l'abdomen et les côtés de la poitrine. appartenir à l'Euphrosyne. Elle nous paraît accidentelle.

Nota. On connait encore plusicurs autres rariétés de Selene, sous les noms de Lycorias, $P$ lintlus, Marphise, $R i$ naldus, Julia, etc., etc. Mais toutes ces variétés qui sont sorement accidentelles, offrent trèpeu d'intérêt.

Envergure, 38 mill. - Ailes entières, Norwége, Laponie, Nous avons vu plusieurs individus de cette espece, tous parfaitement semblables, et elle nous parait distincte de l'A phirape; toutefois on ne pourra trancher enticrement la question que quand les chenilles seront connues.

L'Ossianus est trèsrare dans les collections.

laches légerement nacres; seconde moitié d'un jaune nankin ondé de roux, et ayant au bord terminal un rang de sept taches un peu réniformes, légèrement nacrées et surmontées d'un rang de points jaunes cercles de noir.

o $D^{\prime}$ un fauve plus clair et un peu plus grande.

Envergure, 40 mill. - Ailes entic- Laponic, Suède,

Ellen'est pas ırès-réres, d'un fauve terne, avec la base, les Prusse, Bavière, Livo-pandue dans les collecnervures et de petits points et signes/nie, departements des lions, ce qui nous fai! 
Var. accid. $81 \%$. God. pl. 9 x. fog. $5-4$.

HECA'TE.

Fab. Ochs. Bdr. Hub. 42-4\%.

God. pl. 9 1. fig. 5-6. L'A gare et l'Ino? Engr. rétrécis noirs, et dont ceux de la série| Yosges et de l'Isère? En|douter qu'elle se trouve anté-marginale contigus. Dessous desjuin. effectirement en Frunsupéricures d'un fauve clair, avec le Chenille courte et ce. La série de points le sommet jaune; dessous des inférieu- épaisse, d'un gris ar-des ailes supérieures res d'un jaune citron ondé de roux vers grenté clair, sans ligne est quelquefois pupillée le bord marginal, avec deux bandes dorsale, avec un trait de jaune; ce qui s'obdiscoüdales fauves, dont l'antérieure noir accolé à une ligne serve également chez la peu sinuée, et une séric marginale de plus claire, sous chaque précédente. La chenille chevrons noirs, étroits, contigus et sur-épine dorsale, puis une récemment découverte, montés chacun d'un point jaune cerclélligne latérale blanche; forme une exception rede noir.

$\uparrow$ Plus grande, plus pale et sabléc de noir en dessus. ventre d'un brun noir, marquable dans ce gendepuis le quatrieme re, en ce qu'elle manjusqu'au huitieme an-que des deux longues neau. Epines blanchâ- épines du premier antres, très-courtes, et neau; elle a été trouvée n'elant point plus lon- à terre entre les herbes, gues sur le cou. Tête et on ne connaît pas au petite, d'un gris brun. juste sa nourriture. Se troure en mai.

Chrysalide d'un gris pále. plus clair à la partic postérieure, avec une lizne plus foncée t des taches argentées sur l'abdomen.

Envergure, 42 mill. - Ailes un peu Autriche, Allema- Ce qui distingue dentées, d'un fauve foncé, avec la base ct des taches noires, dont les anté-terminales rondes ou ovales, jamais chene, Russie, midi de principalement cette esla France. En juin. pèce de ses congénères, c'est sa double rangée de points. Le noir du dessus des ailes est souvent un peu chatoyant, surtout chez les femelles, observation qui peut également s'appliquer a l'A phirape.

L'Ino d'Engramelle nous semble appartenir a celte espice, mais sa figure est trop grossière puur que nous puissions l'affirmer.

\section{(92) Genre XVI. MELIT EA (MELITée).}

(Fab. Ochs. Bdv. Dup. Dalm. - Argynnis. Lat. God. (Les Damiers).

Caractères principaux. - Chenille d tubercules charnus et pubescents, d'egalc longueur. - Chrysalide arrondie, obtusc antérieurement, garnie sur le dos de boutons non épineux ct peu saillants, sans taches mélalliques. - Antennes terminées en bouton court aplati ou pyriforme. - Palpes dépassant un peu la tele, velus, dcartés d leur extrémité; leur dernier article terminé en pointe et quelquefois velu. - Ailes n'dtant jamais marquées en dessous de taches nacrées.

Caractẻres secondaircs. - Taille moyenne. - Ailes entières ou dentécs régulicrement, fauves, arec des taches noires formant le plus sourcnt des réseaux. - Abdomen presque toujours aussi long que les ailes inférieures.-Yeux moins gros que dans les ARG YNNEs. (95)

(95) $\{$ Tac

\{Taches noires du dessus des ailes punctiformes et ne formant pas le réscau. .

Taches du dessus des ailes linéaires ou fasciées et formant des réseaux plus ou moins étendus.

(94) Taches noires du dessus des ailes puncliformes et ne formant pas te réseau.

DIDYMA.

Ochs. Bdv.

Envergure, 40 mill.-Ailes un peu| Suisse, Russie, Au-| Elle est tré-commudentées, d'un beau fauterouge, avec la triche, Ilongric, midi, ne dans le midi ainsi God. pl. 4 sec. fig. 2. base, le bord marginal et des taches centre et est de la qu'aux environs de et pl. 4 tert. fig. 5. noirs; celles de la série ante-termina- France. Dans les bois Neufbrisach et de ChatCinxia. Hub. 9-10.', le ordinairement isolées. Dessous des $/$ secs. Fin de juin fleaudun. Nous ne l'a- 
Var. accid. $273-574$. |inféricures d'unbcau jaune citron, avec| Chenille d'un gris/vons jamaistrouvce plus Le Damier. 5' espéce. de nombreux points noirs et deux ban-ardoisé, avec la partie prés de Paris que dans Engr. des transverses d'un faure rouge, dont antéricure de chaque cette dernière localité. l'antérieure bordée de noir des deux anneau plus foncée, Elle vario prodigieusecôtés et la postérieure bordée seulement marquée de points ment, fant pourla taille en arrière de petits ares noirs isolés sur-blancs et garnie d'un que pour l'intensité des montant un rang anté-terminal de points rang d'épines alterna-couleurs et la disposironds de la même couleur. Massue des tivement blanches et tion des taches. antennes terminće par un point fauve fauves a sommité blantres-apparent. Palpes entierement fuuces. che; ventre d'un jaune \& Plus grande, plus pâte et plus ar- pâle. 'Tête et pattes rondie, avec plusieurs rangs de points membraneuses fauves, fauves sur l'abdomen. res. Vit en mái sur différentes plantes, telles que Plantago, Linaria vulgaris, etc.

Chrysalide d'un gris ardoisé, avec des points noirs, le veutre jaundtre et quelques rangées de boutons faures sur l'abdomen.

Andusa. $\quad$ Ne diffère essentiellement de Didyma Mussie, bords du Presque tous les auFab. Ilerbst. God. que par une rangée de points noirs pla- Volga? Bdy. cés sur la bande fauve postérieure du dessous des ailes inféricures.
Trivia.

chs. II ub. 11-12.

Dup. Suppl. pl. 22. fig. $4-5$.

Bdv. Icon. pl. 22, fig. 1-2.
Athulia et Fascelis. Fab. Didyma, rar. God. Encyclopidie.

Le Damier. Lingr. 29 a. b. c. d bis. i non g. h.).
Envergure, 36 mill.-Ailes un pen Piémont, Hongrie, , commune dans les coles chaudes et séches. lections; elle varie asnoires dont les anté-terminales conti- En juillet et noat. sez, surtout pour la tailgues et découpant un rang de lunules Chenille d'un gris le. Godart fait observer de la couleur du fond; inférieures ayant blane, arec plusicurs que les diférences qui le bord alsdominal et les nervures noi- lignes longitudinales séparent les chenilles res. Dessous des inféricures d'un jaune et plusieurs points par- des Mel. Trivia et Dipâle, avec de nombreux pointsnoirs et lie brunatres, partie dyma sont bien minudeux bandes fauves dont la postéricure bleuatres et plus fon-tieuses: " $O$ n en trouve, bordée des deux cótés d'arcsnoirs et sur- cés sur les cótés; épi- dit-il, de plusfrappantes montant un rang anté-terminal, de nes à base rousse, al dans celles su $\boldsymbol{b}$. Petit points noirs triangulaircs. Massue des sommité blanche, ci- paon. "Mais elles n'inantennes noire, avec un petit point a liées de noir. Tête rous- fluent pas, comme ici, peine distinct d'un fauve terne au sommet. $\mathrm{se}$, avec des points (l'une manicre constante Palpes noirs intérieurement. noirs، Pattes blanches, sur les insectes parfaits. † T'antôt de même taille, tantôt plus tachées inférieurement Ces deux espèces sont grande, du méme fauxe que le mâle, plus de noir. Vit en société donc bien distinctes. arrondic et ayant l'abdomen dépourrulau mois de juin sur le Nous avons vu un inde points faures. Verbascum nigrum et dividu de Fascelis Fab. quelquefois sur le $\boldsymbol{V}^{\prime}$. au pas de Suze; il ne Thapsus. diffère pas essenticlleChrysalide d'un gris ment de Trivia. bleuâtre, très-ponctuéc de noir, avec des points orangés sur l'abdomen et sur la tête.

(95) Taches du dessus des ailes linéaires ou fasciées et formant des réseaux plus ou moins ctendlus.

CINXIA Ochs. Bdr.
| Envergure, 40 mill.-Ailes un peu| France, Ilongrie, Elle est très-commudentées, d'un faure terne, réticulées|Suisse, Autriche, Suè-|ne dans les bois. Sa 
God. pl. 4 quart. fig. 1/de noir; inférienres un pou aiguës à|de, etc., etc. Dans les/chenille est également l'angle anal, ayant le deuxieme rano bois. En mai, juin et très-facile ì rencontrer:

Dclia. Fab. Hub. - -" anté-terminal de taches fauves marqué aont. Delia. Fab. Iub. F-".
Le Damier. $4^{e}$ espèce.
Engr. inférieures ayant l'extrémité d'un blanc les incisions marquées qui soit dans ce cas. plus ou moins jaunatre, arec quelques de points blancs. Têle Cette Mélitée varie peu. points noirs et deux bandes d'un faure et pattes membraneuterne, bordées de noir, dont l'antéricure ses d'un fauve rouge, très-sinueuce et la postérieure marquée épines et pattes écaild'une série de petils points noirs.

o Semblable mais plus grande.

leuses noires. Vit en société au mois d'aoat, septembre et avril, sur les Plantago, l'Hieracium pilosella, etc., etc. Passe l'hiver sous une tente soyeuse, et se change a la fin d'avrit en une chrysalide d'un brun jaunatre, piquée de noir et ayant sur le dos plusieurs rangs de tubercules faures.

PIIOEBE.

Fab, Ochs. Bdy Hub. 15-14. God. pl. 4. fig. 2, et 4 quint. fig. 5.

Le grand Damier. Engram.

Melaxina. Luc. Bonaparte.

$P$ hrebe var. Dup. Sup plem. p. 145

ETIERE IIub. $8-5-8,8$.
Envergure, 40 mill.-Ailes d'un fauve-jaunatre pâle, variées de taches $d^{\prime} u n$ fauve roux et réticulées de brun; les supérieures sinues au bord marginal, les inférieures légèrement dentées et ayant le bord terminal brun, entier, surmonté d'une séric de taches jaunâ-
tres lunulées, puis d'une autre de taches d'un faure roux, arrondies. Desches d'un faure roux, arrondies. Des- violet. Dessous du ven-
sous des inféricures d'un jaune très- tre et pattes membra clair arec des points ou traits noirs a la neuses d'un gris rous hase et deux bandes liscrées de noir dont l'antéricure fauve, très-irrégulière excepté celles qui sont En Suisse, elle donne et longée extéricurement par une ligne placées sur la bande en juillet et n'habite noire interrompue, la postérieure d'un fiuve et qui sont deque les cantons mérijaune plus foncé que le fond et marquée celte dernière couleur. dionaux.

de srosses taches rousses tris-rondes; bord Vit en mai et septemterminal longé par un filet noir, a peine bre sur la jacéc Centausensible, sourent nul, surmonté d'une rea jacca.

séric d'arcs de la même couleur.

q Beaucoup plus grande et plus arrondic.

Diffère en ce que les taches jaunes du dessous des inférieures sont remplacées par des taches faures et par deux bandes d'un brun rouge foncé, l'une médiane et l'autre terminale.

Le fond est d'un faure plus foncé et uniforme, c'est-it-dire qu'il n'est point ou à peine varié de roux; les taches du|
Chrysalide d'un gris violâtre, marbrée de brun, avec les incisions brunes et plusieurs rangs de tubercules orangés.

Italic.

Nous exirayons de l'ouvrage de M. Duponchel ce qui est relatif a cette varieté, que nolls n'avons vue ni en nature ni en figure.

Sicile.

M. Duponchel décrit dans son supplément une sous-variété chez

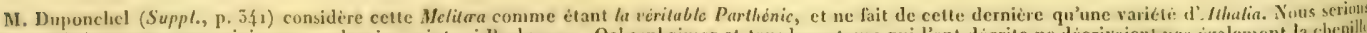
tres-pertes à partager son opinion sur ce dernier point, si Borhausen, Ochsenlecimer et tous les auteus qui l'ont decrite ne décrivaient pas igalement la chenille qui semblesait dillerer beaucoup de celle d'Athatia; mais nous avons di respecter le temoirnarge de ces auteurs et considérer cemme distincte la Parthinic, mal.

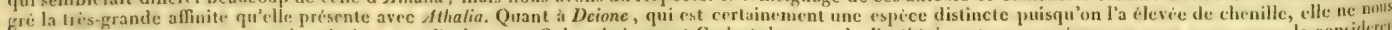

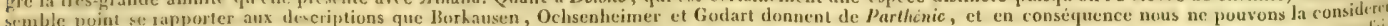

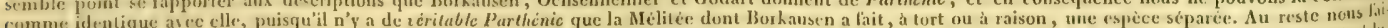

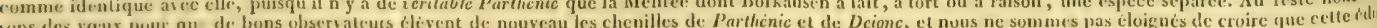

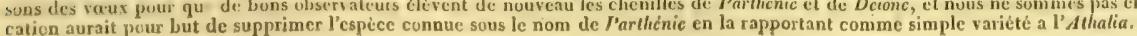


(Dup. Suppt. 1). 44. disque sont plus pelites, plus rares et fig. 4-5. forment plutôt des traits ou points noirs que des réscaux. Une des bandes média. nes brunes est convertie sur les quatre ailes en une série de points contigus ou isolés; en général le brun domine beaucoup moins que chez P/abe; les supérieures sont plus arrondies, moins sinuces au bord terminal. Jeur dessous se rapproche de celui d'Athatia; celui des inférieures ne differe pas sensiblement de Phabe. Quelquefois seulement la ligne fauve qui précéde intérieurcment la deuxième bande est très-large.

DEIONF. Ilub. $9475-950$. Dup. Suppl. pl. 44. fig. Envergure, 35 mill. - Ailes $d^{\prime} u n$ fauve-jaunatre pâle, variées de taches $1-3$.

fauves a peine plus foncées et réticulces de brun; les supéricures arrondies an Parthénic. id. id. p. 341. bord terminal; les inférieures légèrement dentées, avec le bord terminal brun, souvent coupd d'une légere ligne fauve et surmonté d'un rang de taches lunulées, puis d'un autre de taches plus loncées, presque carrés, et ayant le bord abdominal d'un fauve clair. Dessous des inféricures d'un jaune très-pâle, arec les nervures brunes et deux bandes liserées de noir, dontl'antéricure très-irrégulière, d'un fauzc pâte, la postéricure d'un jaune clair, marquéc d'un rang de taches faures, arrondies, contiguës au liscré inféricur. Bord terminal longé par deux lignes noires parallèles.

o Semblable au mâle, mais un peu plus grande.

PAHTHENIE.

Envergure, 35 mill. - Niles légèrc-

France centrale et Cette espèce, trèsment dentées, faures, avec la base et de méridionale, Allema- voisine d'Athalia, s'en lrís-lèger's réscaux noirs. Dessous des gne, Russie, etc., etc. distingue par sa taille inféricures d'un jaune pâle, avec deux Dans les bois secs et constamment plus pebandes faures liserées de noir, dont ćlevís. En juin et aoat. tite, sa coulcur toul'antéricure courte, très-sinuée, joignant Chenille noire, arec jours bien moins charune troisième à la base, et longée du de très-petits points gée de noir en-dessus, cốté opposé par une ligne noire assez blanes à peine distincts, et ses palpes. Nous l'aécartíe, et la postéricure doublement quelques poils fins de vonspriseabondamment liserée de noir en dessus et renfermant cette couleur et une près du Hârre, dans la des espaces plus clairs; frange précédée série latérale de taches Sologne et sur le somd'une ligne un peu sinuée, de la conteur jaunatres faiblement net des Pyrénées; Godu fond, entre deux filets noirs; palpes exprimées. Vit sur le dart nous semble avoir faures endessus. ㅇ Semblable.

Ochs, Bul. Ochs. Bdy.
incnt dentées, arrondies, d'un brun noir, et 4 quint. fig. 2 . Maturna. Fab. Hub. $17-18$.
Le Damier. $5^{\circ}$ espèce. Engr.

France méridionale. Cette espece interest noir chez Parthenie,

liork. Ochs. Dalm. Bd

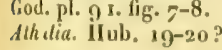

avec de nombreuses taches d'un faure Chenillenoire, semée Lille varie beaucoup, uniforme, disposées par bandes très-ap- de points blanes dans surtout pour l'intensité purcntes et assez larges sur lesquatre ailes. Ies incisions, hérissée de la couleur, ou l'on Dessous des inférieures d'un jaune pâle de poils nombreux, voit dominer tantôt le Ou blanchâtre, arec deux bandes faures blancset noirs, arec les faure et tantôt le noir. liserées de noir; la supéricure large, se tubercules nombreux, Sa chrysalide est une Chrysalide obtuse, taille qui la sépare petite, d'un gris cen-d'Alhatia, du moins de, aree deux rangs n'arons-nous jamais ferrugineux trouvé d'individus aussur le dos. Dans toute l'Europe. Elle est très-commuDans toute l'Europe. mediaire entre Phabe
et Parthenic se distingue facilement de la premiere par sa exprimés en italique, et de la deuxiéme par son faure plus clair, ses ailes plus oblonnal des inférieures, qui etc. Elle est bien caractérisée et sa chenille est connue, quoiquesa cncore été publiée. Le papillon est jusqu'ici très rare dans les coltrés-rare

laquelle le dernier caractere que nous signa-
lons dans notre descriplion est très-prononcé.
Elle a été trouvée dans la lussie méridionale. réunissant à une troisieme à la base, et gros, coniques, d'un fau- des plus jolies parmi longée du côté opposé par une ligne ve clair, les pattes noi-celles des diurnes; noire, peu écartée; l'inférieure plus|res et la tête faure. Elle nous avons plusieurs| 
Jétroite, doublement liserée de noir su-|vit sur diverses plantes, fois élevé la chenille, périeurement et marquée de lunules plus mais surtout sur le qui n'est pas très-rare. foncées, hormis près de la côte; frange Plantago et le Melamprécédée d'une ligne sinuée, un peu plus pyrum sylvalicum. On foncie que le fond, entre deux filets la trouve dans le counoirs. Palpes noirs on dessus. o Semblable.

rant de mai et de septembre.

Chrysalide d'un blanc jaunatre, arec des taches noires et fauves sur l'enveloppe des ailes, cing rangs de petits tubercules peu sensibles, lićs par des bandes faures et bordés de points "noirs sur l'abdomen, et deux taches fauves en forme de $\mathbf{C}$. sur le corselet.
Pxnoxis. Hub. 585 \& 588 . quelqques points dans la cellule ; en des-

ApIIEA.

Hub. 758-750.

Alphera. (var. Alha-

lia). Bdr. Index.

Dalm

Uекта.

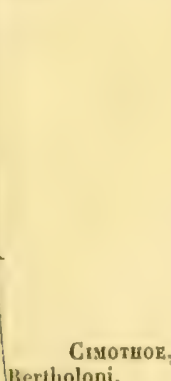

DICTYNNA.

Ochis. Bdy.

God. pl. 4. fig. 3 et pl. 4 quint. fig. 4 .
Le fauve remplit tout le disque aux supérieures, et ne forme aux inférieures qu'une bande anté-marginale avec sous la première moitić desquatre ailes est noire, avec quelques espaces fauves; la seconde est fauve aux supérieures, et aux inférieures clle est jaune avec une seule bande, simple, courte, étroite, fauve; la ligne sinuée terminale n'est point filetée de noir supćrieurement.

o Plus sombre et ayant au-dessus de cette derniere ligne une bande d'atomes noiratres.

Mêmes localités.

Elle ne differe d'Athalia que par'ses taches noires, qui sont confondues endessus de maniere a former des bandes inégales. Le dessous n'est que légèrement modifié.

Ailes un peu dentées, brunes, supérieures, aree une bande maculaire, inférieure, avec une série de points, faures.

(Traduct. de Dalman, pag. 77).

Suèdc méridionale.

Elle ressemble, dit Dalman, à une variété de l'A thalia, figurée par Herbst. ; c'est ce qui nous a engagés d la placer ici, car nous ne l'avons vue ni en nature ni en Ggure. Il décrit aussi, pag. 27 , une autre Mélitée sous le nom de Fulla; mals nous ne savons a quelle espèce la rapporter, d'après sa description, qui est trèsvague.

Italie.

M. Duponchel la regarde comme une variété d'Alhalia, trìsrapprochée de la Pyronia, Ilub. Nous ne l'arons pas rue.

Envergure, 38 mill. - Ailes légèrc- Environs de Paris, ment dentées, d'un noir brun, avec Nord de la France, la seule localité des endes taches disposées par bandes, fau- Suisse, IIongrie, Au- virons de l'áris où nous ves et étroites sur les supéricures, triche, dans les bois ayons pris cette Mélitée, 
Curylhatia. Hub. 15-16. thes-petiles et d'un fuuve blanchâtre sur|couverts. En juin. qui n'yest pas très-rare. Le Damier. 6' espéce. Ifs infirieures. Dessous des sujéricures Chenille d'un brun Il cn est de même dans Lingr.

d'un fuuve brunâtre, avec des points violitre, ponctuéc de la forêt de Mormâle, noirs et l'angle apical jaune. Dessous gris bleuatre, avec les mais elle cst généraledes inféricures d'un jaune sale ou blan- cines un peu plus pâ- ment peu répandue. châtre, avec deux bandes d'un fauve les à sommité noire, et Quelquefoisla ligneterbrunatre, liserées de noir; la supé- troislignes longitudina- minale du dessous des rieure très-sinuée, se réunissant à une les noires; paltes de la inféricures est seulement troisieme à la base et longée du có-couleur du corps; tête teintée de fauve, mais té opposé par une ligne noire: l'infé- noire, avec deux taches cela n'arrive jamais i ricure trè-large et renfermant une d'un gris bleuâtre. Vit l'Athatia. Sourentaussi série de lunules rousses bordées su- en mai, sur la Veronical l'espace compris entre périeurement de noir, et marquées au $A$ grestis. milieu đ'un point noiv' éclairé de jaunâtre; frange précédée d'unc ligne sinuée fauve, entre deux filets noirs; une tache triangulaire il l'angle anal, mi-partie de jaune clair et de fauve brun.

o Plus arrondie et un peu moins obscure en dessus.

la premiere bande fauve et la ligne noire qui la suit est plus foncé que le reste de l'aile $\mathrm{ct}$ découpe ainsi des taches blanchatres; mais on observe la même chose chez plusicurs autres Mélitées. C'est surtout pour l'intensité du fauve que la Dictynna est sujette at varier. Les individus des Alpes sont ordinairement moins rembrunis que ceux de nos enrirons.

CYNTIIA. \begin{tabular}{ll|l} 
Ochs. God. & d'un brun noirâtre, avec destaches d'un voie, sur les hautes cette espèce sont très-
\end{tabular}

Envergure, 40 mil. - Ailes entières Suisse, Tyrol, Sa- Les deux sexes de Hub. 608-609. 569- beau blane et des taches faures, dont montagnes. En juillet dissemblables, ainsi 5 7o. et Mysia. $9^{3} 9-$ la seconde rangée d̀ partir du bord
$944.945-946$. terminal formant une bande étroite, d'aoot.

Dup. Suppl. pl. 21. composée de taches toujours arrondies, Chenille d'un jaune la deseription. Les mâfig. 3-5. Pl. 21. même aux ailes supérieures arrondies, Chenille d'un jaunelles varient beaucoup, Bdv. Icon. pl. 22. fig. y sont visibles, et le plus souvent mar-pâle sur les côtés, plus les uns ont beaucoup 3-5. quées aux inféricures plus souvent mar-pâle sur les côtés, avec de taches faures, d'auLe Damier o ques aux inferieurestepetitspointsnoirs. une ligne noire qui si- tres en sont presque enblanches. Engr. sâtre, arec trois bandes d'un jaune pâ- épines noires; tête d'un mais celles de l'intérieur le, liserées de noir, dont l'antérieure brun roige. Vit sur le des cellules et de la séirréguliere et interrompue, l'anté-mar-Plantago lanceolata. ginale lunulée, et la médiane large et divisée par une ligne noire qui la coupe par la moilié seulement prés de la cóte. Antennes ì massue un peu oblongue et presque entierement noires en dessus.

f Beaucoup plus grande, plus arrondie, ayant le fond d'un lauve jaunâtre, réticulé de noir, avec des taches d'un fauve plus foncé, répondant à celles du male.

La couleur brune est plus pâle et un peu transparente; les taches blanches Dalman.

Maturna. IIub, $80 \%-$ 808 .

in ?. 600-601? sont remplacées par du jaunatre trèsclair, et forment, ainsi que les taches fauves, des séries bien continues et coupcies sculement par lesnervures; les inférieures sont toujours depourvues de points sur les taches fuuves; ces caractéres se répètent en dessous; la ligne noire de la bande médiane des inférieures (quand elle existe) la coupe bien par la moitié dans toute sa longueur, et in bande faure qui la suit est bordée de noir des deux côtés. Ĺn genéral. Ie dessin est plus net et micux marqué, rie des inféricures manquent très-rarement. Elle n'est pas très-rare dansles localités qu'ellc babite.

Laponie méridionale. Cette Mélitée scmble au premier coup d'ocil bien distincte de Cynthia, et devra probablement former par la suite une espèce séparée. Le principal caractère invoqué par Dalman pour la séparer de Maturna (l'absence de la ligne noire sous les inféricures) n'est pas constant, ainsi qu'on peut le roir par notre description. Elle a d'ail-

.




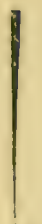 \\ ICINEA. 5-6. \\ MATURNA. Lin. Ochs. God. Bdv. Icon. pl. 25 . fig. 5-́́.}

Dup. Suppl. pl. 22. fig. 1-3.

Cynthia. IIub. 1-2.

Le Damier d taches fauces. Engr.

IIn). 5.

\section{$M Y-1 \mathrm{~A}$.}

H.

Matrinka.

Hub. 598-509. |surtout en dessous, oì les nervures sont aussi plus noires. Enfin la ligne terminale fauve est bien marquée de part et d'autre.

Differe de Cynthia en ce que les deux sexes sont scmblables. Le mâle ressemble à la femelle de celle-ci; mais il est plus foncé, d'un fauve plus uniforme, le brun y domine darantage, surtout sur les inférieures, ou les bandes médiane ct anté-termirale sont presque effacées; la bande jaune antéterminale du dessous des inférieures est plus étroite, et composée de tuches plus réniformes; la bande médiane est plus étroite, et les points noirs qu'on voit sur la partie fauve sont beaucoup plus gros.

Encore plus obscure et plus arrondie.

Envergure, 43 mill. - Ailes entières, les supéricures aiguès au sommet; les

Suède, Laponic, Laponie boréale. quatre d'un brun noirâtre, arec des grie, Liyonie, Russie, bandes, dont l'anté-terminale compo- En juin.

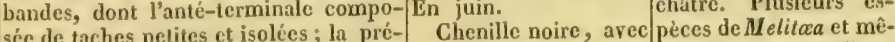
ce de taches petites et isoles, la pre- Chenille noire, arec peces de Hellica et mecedentes tivisées seulement par les ner-d'unjaune soulire, dont le même accident. Le vures, et la précédente composée en la dorsale diviséc par véritable nom de cette grande partie de taches jaunes, dont une ligne noire; tête mélitéc est Manturna, les plus grandes et les plus claires à la et épines noires. Vit en ainsi que l'écrit Dalman, cûte des supérieures. Dessous des infé-mai sur les Plantago, la mais le nom de $\boldsymbol{M}$ aturricures d'un fauve rouge trís-vif, arec Scabiosasuccisa, et même na existant depuis long-: trois bandes jaunes liserées de noir, dit-on, sur le tremble; temps, nous ayons cru trantéricure très-interrompue, mais cette dernière as- devoir le laisser subsisl'anté-terminale étroite et lunulée, et sertion mérite confir-ter. Elle n'est pas comla médiane régulière, d'égale largeur et mation, aucune espèce mune. On a dit qu'elle sourent divisée par une ligne noire qui voisine ne virant sur se trouvait dans le déla coupe par moitić dans toute sa lon-les arbres. partement de l'Isère; gueur. Antennes ayant le sommet de Chrysalide jaunatre mais il n'est pas à noia massue larmement faure.

o Plus grande, plus arrondie, avec de noir. la deuxième bande anté-terminale encore plus large.

C'est une variété dont plusieurs des taches discoildales sont blanches.

La troisieme séric anté-terminale est entièrement blanche aux supérieures, ct grisâtre aux inféricures; on voit aussi sur les premières deux autres taches blanches, dont une dans la cellule et une au bord interne. La deuxieme sé- ou verdatre, ponctuée tre connaissance qu'elle y ait eté prise. Les nôtres viennent de la province du Bannat, en IIongric.

Nous avonstu en nature des variétés de Maturna, qui sc rapprochaient beaucoup de cette figure; nous croyons done que c'est ici qu'elle doit se rapporter. Il n'en est pas de même de Mysia, $939-944$, etc.

Nous n'avons pas vu en nature cette remarquable variété. Nous ne sommes done pas certains qu'elle doire se rapporter à Maturna; 
frie anté-terminale de taches fauves des inférieures est plus étroite et placée beaucoup plus haut; le dessous des premières ailes se rapproche de Cynthia, celui des secondes ne differe de Maturna qu'en ce que sa bande anté-termina-

DESFONTAINII. God. Encycl. Desfontainesi. Bdv. pl. 23. fig, $1-2$.

ARTEMIS.

Fab. Ochs. Bdr. Hub. 4-5.

God. pl. 4 sec. et 4 tert. fig. 3.

Le petil Damier a ta ches faures. Engr.

Menope,

De Prun. Dup. Suppl. pl. 21. fig. 1-2. Treits. Suppl. p. \&.

I.

Envergure, 48 mill. - Ailes entie1. de jaune et réticulées de noir, avec bande anté-marginale de couleur plus vive; cette derniere marque d'une série de points, jaunes sur les supérieures, noirs. nières ayant le disque noir, avec beancoup de taches irrégulières de la couleur du fond, dont les externes plus claires et disposées en bande; dessous des mêmes ailes d'un rouge fauve, avec trois bandes d'un jaune clair liserées de noir, la premiere droite et accompagnée d'une tache au milieu, la seconde courbe et s'étendant a l'angle anal, la troisième terminale, lunulée et surmontée d'une série de points noirs cerclés de jaunâtre.

o Plus grande, plus claire et à ailes supérieures plus arrondies. Le fauve et le jaune y sont plus tranchés.

Envergure, 35 mill. -Ailes entières; points noirs cerclés de jaune.

res plus arrondies. le est blanche.

elle pourrait appartenir à Cynthia. Quant it la femelle, représentée sur la même planche ( nos 600 et 601 ), elle nous parait lien plus voisine de l'/duna, ainsi que nous l'arons dit d son article.

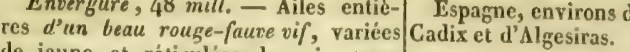
deux taches dans la cellule et une large sur les inférieures. Dessus de ces der-

Nous avons ru une paire bien conservée de cette Mélitice, qui nous semble assez distincte d'Artemis. Le mále surtout se rapproche davantage de $\mathbf{M} a$ turna; la rangée antéterminale de points jaunes sur la bande des supérieures y est très-peu apparente, et il est probable qu'elle doit quelquefois s'étioler tout-d-fait. Ce n'est donc point lì, comme le pretend M. BdY., le caractère qui séparc cette espece d'Artemis chez laquelle cette rangée de points existe quelquefois d'une manière très-prononcée. Nous ignorons aussi pourquoi cet auteur a changé le nom qui arait été imposé à cettc Mélitée par Godart, qui l'a fait connaitre lc premier. Nous le lui arons restitué. les supéricures un peu anguleuses, les Dans presque toute Elle est très-commul'Lurope, dans les bois. ne dans les bois un peu inférieures arrondies, les quatre d'un En mai et aoot. étendus. Sa chenille, fauve rougeâtre clair, variées te jature et
Chenille noire, avec qu'on confondrait d'aréticulées de noir; supéricures ayant la trois bandes longitudi-bord aree celle de deuxième bande anté-terminale étroite, nales et maculaires de Cinxia, en diffère maculaire et souvent marquée de points composées de petits par satête, qui est fauve jaunes; inférieares ayant la même points blancs; pattes chezcette dernicre. Le bande large, continue et marquée $d^{\prime} u n e$ membraneuses fauves. pajpillon variebeaucoup, série de points noirs. Dessous des infé- Tête et épines noires. surtout pour les courieures d'un faute roussâtre pâle, avec Yit, en société dans le leurs du fond, qui sont trois bandes d'un jaune très-clair, lise- jeune âge, sur la Scabio- sourent confondues rées de noir, dont l'antérieure très-ma- sa succisa. En arril, ensemble. culaire, la médiane étrojte et n'étant juillet et septembre. traversée d'aucune ligne, et la posté- Chrysalide d'unblanc rieure terminale, traversée d'une petite jaunâtre, avec des taligne noire et surmontée $d^{\prime} u n$ rang $d t$ ches noires et des ta-

Plus petite : toutes les taches, a l'ex- Hautes Alpes de la ception de la séric quí aux inférieures et deux ou trois autres Suisse, de la Savoie, sanne, nous mande que aux inferieures et deux ou trois autres du Piémont. En juillet celte espèce différe it la base, sont d'un jaune pâle et com- et aoot
me étiolé.

ensemble.

M. Bugnion, de Lau-
sanne, nous mande que
celte espece difrere
d'Artemis par son vol
et ses moeurs, qui res-


Bdr. Icon. pl. 22. fig.
G-7.
Artemis. Hub. 653.
Proviscists.
Bdv.

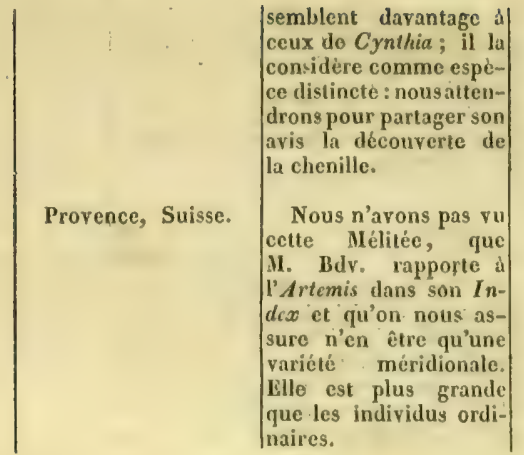

(96) Chenilles completement dépourcues d'ipines ou de tubercules, ct ayant l'extrémité de l'abilomen diviséc en deux pointes *. - Cellule discoidale des secondes aites toujours fermée. .

\title{
(97) Genre XVI. SATYRUS (SATYRE).**
}

\author{
(Lat. God. - Hipparchia. Ochs. Tr. - Erebia. Dalm. - Satyrides (tribu des) Bdv.)
}

Caractères principaux. - Chenille assez courte, un peu renfle au milieu, trés-altinuée postéricurement, ayant l'anus garni de deux pointes formant une petite fourche. - Chrysalide assez ramasse, peu anguleuse ou tout-d-fait obtuse. -Palpes assez grèles, hérissés de poils en avant, comprimés latéralement et dépassant le chaperon.-Antennes de forme variable.

Caractères secondaires. - Chenilles vivant cxclusivement de gramines, presque toujours rugucuses et pubescentes, quelquefois rases. Tuille variable. - Ailes assez arrondies, souvent dentées; les supericures ayant a l'angle apical un petit ceil visible en dessous, plus ou moins apparent en dessus, et tres-souvent l'une ou l'autre des principalcs nervures renflée d sa base; les inferieures ayant en dessous trois lignes plus ou moins visibles (quelquefois effacées), ct dont les deux intérieures forment souvent entre elles une bande plus foncée ${ }^{* \star \star}$. - Vol irrégulier, peu soutenu. - Ailes supérieurés plus oi moins cachées par les inférieures dans le repos. .

(Chenille pubescente. - Nervure costale seule renlée a Ja base des supérieures. - Antenncs épaisses, longues, Uroites, à massue grossissant insensiblement et fusiformic. - Ailes dentées, blanches, avec des dessins et taches noirs. - Taille un peu au-dessus de la moyenne.

Chenille pubescente. - Aucune des trois nervures des supérieures n'étant bien sensiblement renflée à la base. - Antennes grêles, à massue oblongue, ovale, aplatie. - Ailes rarement dentées, arrondiess, d'un brun foncé ou noir, ayant presque toujours de larges bandes anté-terminales d'un roux ferrugineux, chargées de points ou d'yeux noirs. - Tuille

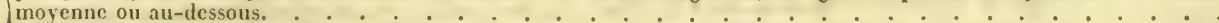
Chenille glabre. - Nervure costale très-renflée a la base, la médiane seulement un peu dilatéc.-Antennes grêles, à massue pyriforme.- - Ailes arrondies, d'un brun-noir uni dans les mâles. - Taille au-dessus de la moyenne. Chenille glabre. - Nervures costale et médiane également très-renflées à leur base; couleurs variées. - Taille moyenne et au-dessus.

\footnotetext{
- Nous devons faire observer que, dans le genre suivant, les Chrysalides ne sont pas toujours suspendues par la queue. Quelquefois elles ne sont fixées par aucup lien et posées immédiatement sur la terre sous des touffes d'herbes, quelquefois mème elles sont enterrées peu profondément. Ces espéces forment donc une ex. ception dans les Tymphalides, comme le genre Parnassius dans les Papillonides, et nous fournisscut de nouveau la preuve que la nature se refuse à toute espiec de classification absolue.

- Nous aurions voulu pouvoir adopter quelques-uns des genres qu'on a créés aux dépens de celui si nombreux des Satyres; mais ces genres, appuyés seulemeal sur la conformité du facies des insectes parfaits, nous ont paru avoir des bases trop peu solides, et nous nous sommes bornés, a l'exemple de M. Duponchel, à diviser le genre Salyrus en groupes dont nous lui avons emprunté la majeure partie, sans pour cela les caractériser toujours comme lui,

*** Pour abreger le plus possible nos descriptions, nous nommerons ligne basilaire celfe qui est la plus rapprochée de la base, ligne médinne celle qu'on remat que vers le nilieu de l'aile et qui est souvent celaire de blanc, et ligne ante-ferminale cclle qui longe le bord terminal et qui est souvent interenpue. Ces lignes deterninent presrue toujours le's dessins Jes ailes inférieures, $\mathrm{ct}$ avec un peu d'altention un en retrouvera la trace chez presque tous les Salyrus. La basilaire est la seule qui disparaisse parfuis complétement. (Voyez la planche explicative.)

"..* Ces deux divisions sont très-artificielles et souffrent quelques exceptions, surtout dans certaines femelles de Nègres (Erebia. Bdv.). Nons ne les doanons done que comme un moyen de scinder un peu les groupes nombreux qui composent ce genre, afin d'ea rendre la recherche plus facile aux commençants.
} 
(101) Chenille pubescente. - Nervure costale (des supérieures) seule renfléc de la base. - Antennes epaisses, longucs, droites, à massue grossissant insensiblement et fusiforme. - Ailes dentées blanches, avec des dessins et des taches noirs. - Taille un peu au-dessus de la
moyenne.

Genre ARGE. Bdy. - Hipparchia. fam. D. Ochs. - Satyres Leucomelaniens. Lefebv. - Les Graminicoles. Dup. Satyres blancs vulgairement.

(102) \{ Ligne noire terminale des secondes ailes n'étant jannais double. . . . . . . . . . . . . . (105)

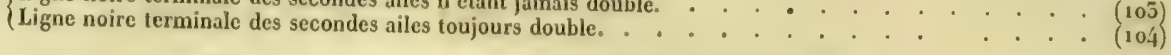

(103) Ligne noire terminale des secondes ailes n'étant jamais double.

LACHESIS.

Ochs. Bdv,

Hub. $186-189$.

God. pl. 19 s. fig. 1-2. Le Demi-Deuil. var. Engram. fig. 60 e. f. pl. 30.

GALATIIEA. Lin. Fab. IIub. 18518 年. God. pl. 8. fig. 2. Golatea. Ochs. Bdv. Lo Demi-Deuil. Engr.
Envergure, 55 mill. - Ailes d'un| Sud-Ouest dela Fran- Il est commun dans blanc un peu jaunâtre, avec la base al ce et de l'Europe, envi- les pays qu'il babite. sins noirs. Côte des des- rons de Nimes, de Nous récolté sins noirs. Côte des supéricures blan- Perpignan, etc. En mailabondamment près de che ou seulement un peu grisatre et juin.

dans les deux sexes. Tache du disque des premières ailes nettement exprimée, et figurant grossierement une boule appuyée sur l'extrémité d'un rectangle assez long. Dessous des inférieures avec des dessins gris formant une bande médiane interrompue au milieu, et cing yeux anté-terminaux pupillés de bleu pâle.

Plus grande, et ayant le dessous des inféricures lavé de jaune d'ocre.

Envergure, 47 mill. - Ailes d'un blanc soufre, avec des taches noires IIabite presque toutc la bitse de la même coulcur entourant le secs et herbus. En juin minent plus ou moins commencement de la cellule, qui est de et juillet.

Montpellier. La base de ses quatre ailes le fait distinguer au premier coup d'oil de ses congénères. Godart s'est trompé en disant qu'Engramelle n'a pas connu cette espèce, c'est elle sans nul doute que représente sa figure 60. c. f. $x$

la couleur du fond; tache annulaire ${ }^{\star} n^{\prime} \ell-$.

juillet.
Chenille verte ou constituent les variétés suivant la latitude, et tant jamais arrondie ni évidéc au mi-roussâtre, arec une li- suivantes.

lieu; un petit point noir au sommet gne dorsale plus foncée des supérieures, souvent confus en et plusieurs autres lidessus et parfois ocellé en dessous. gnes latérales semblaBordure noire des secondes ailes bien bles, dont quclquesmarquéc, nettement coupée supérieu- unes liserées de coulcur rement et renfermant les yeux, qui sont pâle, et entre lesquelpeu visibles en dessus, très-visibles les se voit un espace mais fort peu épais en dessous. Bande livide renfermant les médiane du dessous des mêmes ailes stigmates, qui sont roux interrompue au milieu. o Plus grande, et ayant le dessous noir; tête et pattes des inférieures et la côte des supéricu-ćcailleuses roussâtres, res lavés de jaune d'ocre roussâtre. membraneuses de la couleur du fond. Vit en avril et mai sur les graminées.

Chrysalide épaisse, obtuse, à ventre renlé, d'un gris roussâtre, avec l'enveloppe des ailes plus clairc et un stigmate noir très-saillant à leur naissance. Non suspendue et cachée sous des touffes d'herbes.

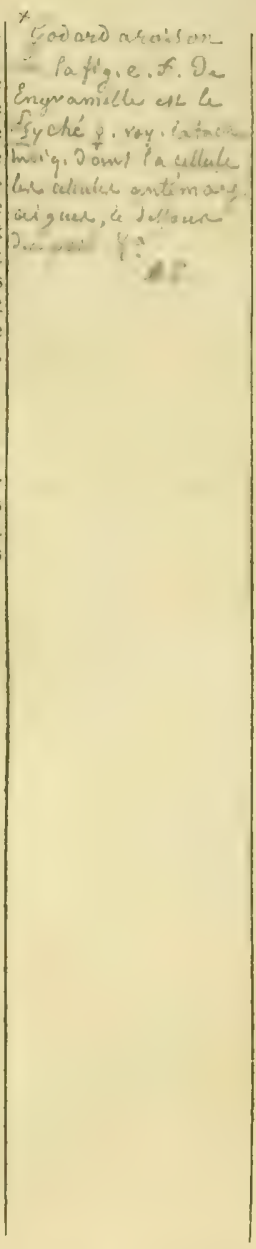

L'Mnsemble de a clonné le non de tache annulaire à celle qui est à l'extrémité de la cellule des supérieures ct qui s'appuie sur une autre fache sourent carree. qua se trouve sur le milicu de l'aile. (Voyez qui se trouve sur le milicu de l'aíle. (Voyez la planche explicative.) 


\begin{tabular}{|c|c|c|c|}
\hline 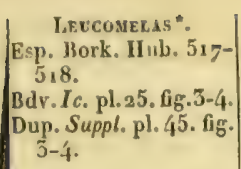 & $\begin{array}{l}\text { Lo dessous des inferieures est d'un } \\
\text { jaunâtre ou d'un jaune ochracé unifor- } \\
\text { me (suirant le sexe), et les dessins y } \\
\text { ont presque complétement disparu, sur- } \\
\text { tout les yeux. Le dessus est sourent } \\
\text { aussi plus chargé de noir. }\end{array}$ & \begin{tabular}{|c|} 
Hongrie, Croatie, \\
midi de la France.
\end{tabular} & $\begin{array}{l}\text { C'est surtout des fe- } \\
\text { melle qu'on rencontre } \\
\text { dans cette variété, et } \\
\text { les mâles sont très- } \\
\text { rares. }\end{array}$ \\
\hline $\begin{array}{l}\text { Galese. } \\
\text { Ochs. } \\
\text { Galatea. var. Ochs. } \\
\text { Tr. }\end{array}$ & $\begin{array}{l}\text { Diffère de Galalhca, en ce que la } \\
\text { bande anté-marginale du dessous des } \\
\text { inféricures est maculaire et sans yeux. } \\
\text { Cette variété se rapproche beaucoup de } \\
\text { Leucomelas; mais ce sont surtout des } \\
\text { matles qu'on rencontre, tandis que c'est } \\
\text { le contraire chez la premiere. }\end{array}$ & & $\begin{array}{l}\text { Elle avait été érigéc } \\
\text { en espèce par Ochs., } \\
\text { qui plus tard la rappor- } \\
\text { ta a Galutea. M. Treits- } \\
\text { chke l'a trouvée plu- } \\
\text { sieurs fois accouplée } \\
\text { avec Galatea ainsi que } \\
\text { Leucomelas. }\end{array}$ \\
\hline $\begin{array}{l}\text { Procids. } \\
\text { Herbst. Hub. 658- } \\
659 \text {. } \\
\text { Bdy. Icon. pl. 25. fig. } \\
\text { 5-6. } \\
\text { Dup. Suppl. pl. } 45 . \\
\text { fig. 5-6. } \\
\text { Galaxera. Esp. }\end{array}$ & $\begin{array}{l}\text { Tantôt de même taille que Galalhea } \\
\text { et tantôt un peu plus grand. Le noir do- } \\
\text { mine bien davantage sur ses quatre } \\
\text { ailes et absorbe presque complétement } \\
\text { en dessus les taches blanches anté-mar- } \\
\text { ginales. }\end{array}$ & $\begin{array}{l}\text { Piémont, Italie, mi- } \\
\text { di et quelquefois cen- } \\
\text { tre de la France. }\end{array}$ & $\begin{array}{l}\text { Nous l'avons pris aux } \\
\text { environs de Chartres. } \\
\text { Les indiridus qu'on } \\
\text { prend dans le fles de la } \\
\text { Grèce et aux environs } \\
\text { de Constantinople sont } \\
\text { particulierement très- } \\
\text { grands et très-chargés } \\
\text { de noir. }\end{array}$ \\
\hline $\begin{array}{l}\text { HERTA. } \\
\text { Dahl. Hub. 900-9o3. } \\
\text { Bdv. I Icon. pl. 28. fig. } \\
\text { 1-5. } \\
\text { Treits. Suppl. p. 39. } \\
\text { Larissa. Lefeb. Ann. } \\
\text { de la Soc. Entom. de } \\
\text { France. pl. 2. fig: } 5 . \\
\end{array}$ & $\begin{array}{l}\text { Envergure, } 52 \text { mill. - Ailes d'un } \\
\text { blanc assez pur, avec la base d'un gris } \\
\text { obscur et des taches noires. Supérieures } \\
\text { ayant a l'angle anal un point noir et } \\
\text { dans la cellule une petite ligne etroite, } \\
\text { filiforme, coutée au milieu; tache an- } \\
\text { nulaire arrondie, plus claire au centre; } \\
\text { lunules anté-terminales des inférieures } \\
\text { grandes, bien marquées et surmontées } \\
\text { de deux ou trois yeux placés sur une } \\
\text { bande noire plus ou moins épaisse et } \\
\text { qui remonte presque sans interruption } \\
\text { jusqu'd la côte. Dessous des supérieures } \\
\text { n'ayant de bien marqué en noir que } \\
\text { l'angle interne et la lignemédiane. Des- } \\
\text { sous des intéricures avec les lignes ba- } \\
\text { silaire et médiane bien marquées en } \\
\text { noir et formant une bande non interrom- } \\
\text { pue depuis la côte jusqu'i l'angle anal; } \\
\text { ligne anté-terminale nette, et surmon- } \\
\text { tée des yeux, qui sont grands et bien } \\
\text { marqués. Antennes noires, a massue } \\
\text { souvent blanchâtre, avec l'extrémité } \\
\text { ferrugineuse. } \\
\text { \& Plus grande, plus jaunâtre et un } \\
\text { peu plus marquée de noir que le male. }\end{array}$ & 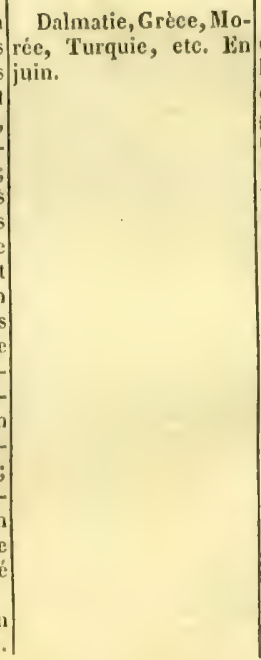 & $\begin{array}{l}\text { Cette espece, connue } \\
\text { dans beaucoup de col- } \\
\text { lections sous le nom } \\
\text { de Larissa, était dési- } \\
\text { gnée depuis très-long- } \\
\text { temps sous celui de } \\
\text { II erta par Dahl, sui- } \\
\text { vant le témoignagne de } \\
\text { WI. Boisduval, qui le lui } \\
\text { restitue. Nous avons } \\
\text { suivi son exemple. } \\
\text { Nora. Plusieurs an- } \\
\text { teurs modernes don- } \\
\text { nent sous les noms de } \\
\text { Darceti, Titea et IIyla- } \\
\text { la, un satyre voisin de } \\
\text { celui-ci; mais ils lui as- } \\
\text { signent tous pour pa- } \\
\text { trie la Syrie et le } \\
\text { Mont-Liban. Il sort } \\
\text { donc tout-à-fait de } \\
\text { notre cadre, malgré la } \\
\text { ressemblance de son } \\
\text { facies avec celui des } \\
\text { copeces de cette sec- } \\
\text { tion. }\end{array}$ \\
\hline
\end{tabular}

- M. Boisduval dit, en pailant de ce Satyre, qu'il est une varicté accidentelle cé constante du Galathea (Icon., p. 133). Sans prétendre blamer aucunement cet entomologiste, nous devons a cette note pour réparer une omission que nous avons faite à ce sujet dans notre préface.

Suivant nous, une variłté accidentellc (en latin abcrratio) est une deviation purement fortuite du type commun, un véritable monstre, qui, tout en s'éloignant quelquefois prudiricusement l'espèce oudie quel

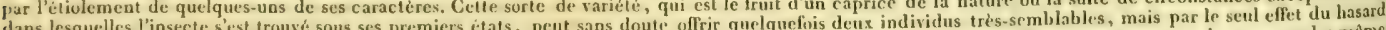
dans lesquelles l'insecte s'est trouvé sous ses premiers etats, peut sans doute offrir quelquefois deux individus tres-semblables, mais par le sent eftet du hasares Une variété constante, au contraire, forne pour ainsi dire une race à part, oflrant toujours les menes caractères et diflërant constamment du type par les méts sont exceptions; elle est due \l'influence du climat ou à d'autres causes suuvent inexplicables, et ne diffëre d'une espéce distincte qu'en ce que ses premifrs etats sone semblables a ceux de l'espece typique; car si elle s'en distinguait aussi constaminent sons la forme de chenille et de chrysalide, elle constituerait pour nous uneespiece séparie, sans que nous cherchions a nous enquérir si ces modifications sont dues al'influence du climat ou à toute autre cause, question que nuous considerons comme purement physiologique.

Il résulte de ce que nous venons de dire: $1^{\circ}$ que nous ne saurions qualifier un Jipideptère de varietét à la fois constante et accidentelle, comne le fait M. Boisdu-

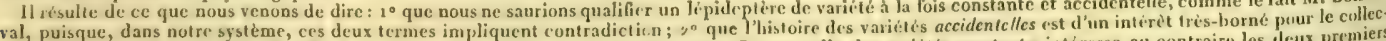
leur et liconorgaphe, tandis qu'il offre au contraire un vasle clamp au pliysiologiste; $3^{\circ}$ que celle des varietés constantes interesse au contraire les deux premiers au wlus haut point, puisque de la solution des questions qu'elles font naitre depend la fixation invariahle des especes, Ces principes sont ceux qui nous guidero aus le courant de cet ouyrage, et il était d'autant plus gecessairc de les expliquer ici, qu'ils different a quelques égards de ceux adoptés par plusieurs icono graplies. 


\section{G. SATYRUS.}

\begin{tabular}{l|l} 
LaRrssa. & Les ailes sont un peu plus jaunatres \\
Pareyss. Lelebv. IIub. & et plus chargées de noir en dessus. Cet-
\end{tabular} $896-809$. te couleur envahit presque totalement Bdv. pl. 28 . fig. 4-6. les lunules anté-terminales, ct en desDup. Suppl. pl. 26. fig. sous des supérieures la ligne qui précè$1-4$.

de l'anté-terminale est presque aussi marquée de noir que la médiane.

CLotı. Envergure, 60 mill. - Ailes d'un ochs. I.efebr. Hub. blanc pur ou légèrement jaunatre, Piémont, Calabre

Mêmes localités.', Herta ce que Procida est is Galalica, et se fond insensiblement avec lui par des individus intermédiaires.

190-19x. avec la base obscure, et une bordure juin et juille

Dup. Suppl. pl.25. fig. anté-terminale réduite d une simple li$1-4$.

gne et découpant des lunules grandes et

Bdv. Icon. pl. 25. fig. bien marquées; supérieures un peu an1-2.

Arge, Fab. God. guleuses au sommet, où ellessont marquées d'un point noir précédé d'une ligne courte, et ayant une petite ligne en zig-zag dans la cellule avant la tache annulairc, qui est arrondie, évidée au milieu et qui commence une espèce de bande étroite, très-sinuéc et irrégulière, donton voit une semblable sur le disque des inférieures (où elle est souvent coupée de petites taches blanches). Yeux anté-marginaux bicn pupillés et entourés seulement d'un léger cercle noirâtre. Dessous des inférieures ne différant du dessus que par la bande médiane, qui est vide au milieu, et les yeux qui sont bien plus marqués et lavés de jaune; antennes ferrugineuses en dessous et au sommet.

o l'lus grande.

Cleanthe.

Bdr. Icon. pl, 26, fig. 1-3.

Clolho, var. Lefebv, Dup. Suppl. pl. 25. fig. 5-6.

Dahli.

Lyssianassa.

Atropos. Hub. 192193.

Clotho, rar. Lef. Bdy.

Plus petit; base des ailes plus noire t plus couverte de poils, les supérieures moins anguleuses au sommet, ligne en zig-zag plus épaisse et brisée au milieu, tache annulaire des supérieures ayant la partic postéricure en carré long, dessins généralement plus épais; yeux des inférieures saupoudrés de noir à l'entour. Ailes d'un blanc très-pur, rarement jaunatre en dessous.

o Un peu plus grande et plus marquée de noir.

Taille de Lachesis; ailes de même forme que celles de Cleanthe, mais ayant au contraire le fond d'un blanc très-jaunatre; taches noires, généralement plus dilatées en dessus et altérant par conséquent beancoup la pureté de la ligne anté-terminale et la netteté des lunules blanches. Yeux des inféricures très-entourés d'atomes noirs. \& Analogue.
Alpes de la France. Environs de Digne.

Sicile, Calabre.
On distingue souvent aussi dans ce Satyre un petit point noir intermédiaire s'alignant avec les yeux des inférieures tant en dessus qu'en dessous. Il est possible qu'il doive constituer une espèce séparée, ce que la connaissance de sa chenille nous apprendra.

Nous croyons deroir considérer comme identiques le Lyssia nassa de Dahl et l'Atropos d'IIubner. Ils ont un facies assez différent de Clotho, mais on les reconnaîtra toujours au moyen de la ligne en zig-zag des ailes supérieures.

(104) Ligne noire terminale des secondes ailes toujours double.

ARGE.

Envergure, 52 mill.-Ailes blanches, Calabre, Sicile, Italic Cette belle espice Ochs. Ilerbst. Lef. arrondies, grisûtres à la base, avec la li-méridionale. En juin. est celle de tuute la Dup. Suppl. pl. 26. gne anté-terminale très-peu empatée fig. 5-6.

de noir aux supérieures, très-nctte nux inférieures, el découpant des lunules blanches, larges et bien marquées; susection out le blanc domine le plus. Elle est rare dans les collections et offre un assez 


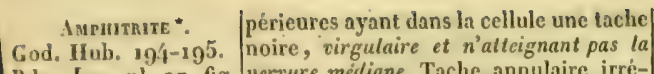
$13 \mathrm{dr}$. Icon. pl. 27. fig. nervure mediane. Tache annulaire irré$1-x$.

gulitre, anguleuse intérieurement, Le Demi-Douil aux évidée au centre et jetant ì sa partie yeux blens. lingr. postérieure un simple rameau sinué, aigu par en bas. Tache noire apicale

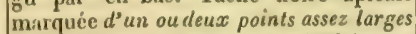
d'un beau bleu. Dessous des inférieures marqué des yeux ordinaires, qui sont roux, pupillés de bleu et cerclés de jaune et de brun, et de deux lignes brunes partant de la côte et formant près du bord abdominal une espèce de parenthèse. Tous ces caractères se répètent en dessus, mais les lignes ne s'y voient qu'en transparence.

o plus grande et encore moins marquée de noir.

Phervsa.

D)ahl.

Bdv. Icon. pl. 26, fig. $4-6$.

Psyche. var. Lefebr.

Arge var. Treitschke.

PSTGHE.
God. pl. 39 s. fig. 5-4.

llub. 198-199.

Var. accid. 676-6-67. $6,6-697$.

Syllius. Och,s.

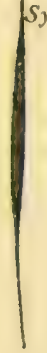
rieures, qui est plus large et se lie arec les précédentes, par la ligne anté-termiet en ce que tous les yeux, tant aux supérieures qu'aux inféricures, sont réduits à de simples points dont les deux de la côte des superieures plus petits. En dessous ce satyre se rapproche beaucoup de $\boldsymbol{P}$ syche, dont il ne differe que parles dessins roux plus étroits, plus pâles, surtout aux inférieures, où toutes les lignes sont bien moins prononcées et ou les yeux sont plus petits et presque isolés.

ㅇ Plus grande, ayant les dessins plus marqués, les yeux plus grands et souvent pupillés en dessus. la tache noire du bord interne des supe-
Diffère d'Arge par le noir, qui domine davantage surtout i la base, par

Envergure, 5o mill. - Ailes un peu nale qui forme deslunules plus aiguës,

Sicile.

Midi de la France. Il n'est pas rare aux Dalmatie, Sardaigne, environs de Montpelayant le bord interne noiratre, une etc. En juin et juillet. lier.

bordure anté-marginale assez large, surtout ì l'angle interne, découpant des lunules petites et inégales, et dans le milieu de la cellule une ligne sinué, terminée supéricurement en crochet et se joignant inféricurement d la tache annulaire, quui est arrondie, bien évidée au milieu et appuyée sur une tache carrée a partie inférieure virgulaire; inféricures ayant la double ligne marginale surmontée d'anneaux noirs, sur lesquels sont les yeux, grands, bien pupillés et saupoudrés à l'entour de noirâtre. Dessous des supérieures avec les nervures, la bordure, les anneaux, deux lignes partant de la côte, deux parenthises au| grand nombre de variétés, soit pour le nombre et la grandeur des yeux, soit pour la netteté et l'intensité des dessins.

II participe à la fols d'Arge et de Psyche. Nous en arons ru quatre exemplaires, deux pris par M. Lefebvre en Sicile, et qui se rapprochaient beaucoup de Psyche, et deux autres enroyés par Dahl et qui ont plus de rapports avec Arge. II nous serait done fort difficile de trancher la question qui partage à ce sujet deux de nos plus sarants entomologistes; mais M. Treitschke, qui a comparé un très-grand nombred'individus, pense que le Pherusa n'est qu'unc variété d'Arge, avec lequel nous devons dire qu'il a beaucoup de rapports, surtout par la ligne intra-cellulaire.

$$
\text { |llet. }
$$

- Les personnes qui adopteront le genre Arge de M. Boisduval derront prendre ce dernier num pour éviter la répétition. 


\section{G. SATYRUS.}

bord abdominal d'un brun ferrugineux, et les yeux très-grands, d'un roux pâle, pupillés de bleu, cerclés de jaunâtre et entourés de brun. Antennes très noires. of Semblable, mais à dessins plus épais.

Ixora. Un peu plus empaté de noir au bord Buv. Icon. pl. 27. 0g. terminal des supéricures et sans yeux

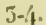

Psyche. Ilub. 694 tont en dessus qu'en dessous. $69^{5}$ ?

INI:S.

Hoffm. Lefeb. Dup. Suppl. pl. 24. fig Envergure, 47 mill. - Ailes blanches, $1-4$. Bdr Icon. pl. 27. fig 5-6.

Thetis. Hub, 196-197. d'un gris obscur à la base; les supéMidi de la Franec et de l'Europe.

Variéte accidentelle pour laquelle on pouvait s'épargner la peine de creer un nom; fig. 694-695 d'Hub. a beaucoup d'analogic arec elle.

\section{Espagne et Portugal. Il est très-rare dans} les collections. très empátce de noir et découpant des . Iunales petites et inégales; milicu de la cellule marqué d'une ligne noire, epaisse, coudéc et anguleuse au milieu et atteignant les deux nervures, contiguë inferieurement a une autre plus fine qui va rejoindre le gris du bord interne. Dessous des supérieures avec des dessins noirs bien marqués, la cóte striée de noir et un ail apical ferrugineux. Dessous des inférieures avec deux lignes noires médianes, dont l'extéricure plus longue et répétée en dessus et cing yeux virement colorés, rouges, à prunelle bleue et cerclés de jaune et de noir; antennes faures en dessous.

\& Plus grande, plus jaunatre et ayant le dessous des inféricures ochracé.

(105) Chenille pubescente. - Aucune des trois nervures des supéricures n'ctant bien sensiblement renfée a la base. Antennes greles, à massuc oblongue, ovale, aplatie. - Liles rarement dentées, arrondies, d'un brun foncé ou noir, ayant presque toujour's de larges bandes antiterminales $u^{\prime} u n$ roux ferrugineux chargées de points ou d'yeux noirs. - Taille mojenne ou au-dessous.

Genre EREBIA. Bdy. - Ifipparchia. fam. E. F. Ochs. - Satyres melaniens. Lefeb. - Ies Alpicoles. Dup. - Satyres nìgres vulgairement. . . . . . . . . . . .

(106) \{rrange des quatre ailes n'étant pas entrecoupée de gris et de noir, du moinș dạns les mâles. . . . . . (10-5)

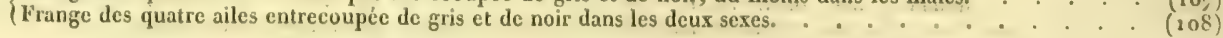

(107) Frange des quatre ailes n'etant pas entrecoupée de gris et de noir, du moins dans les mâles.

CASSIOPE. Fab. Ochs. Bdy. Hub. 626-629. God. pl. 15 o. fig. $1-2$. Le petit Nigre d bandes faures. lingr.
Envergure, 35 mill. - Ailes d'un| Styric, Pyrénées,| Mabite à une assez brun noir, les supérieures avec unc Alpes de la Suisse, du grande élévation et vabande ferrugineuse, un peu maculaire, Tyrol, etc. En juillet. ric assez, principalepeu tranchée, marquée de trois à quatre points noirs non ocellés dont les apicaux plus gros et le troisième rejeté vers le bord; inféricures avec une série de taches petites, arrondies, marquées chacunc d'un point noir non ocelle. Dessous des supérieures ne différant du dessus qu'en ce que les taches ferrugineuses y sont confluentes. Dessous des intérieures arec la base plus foncée jusqu'audeda du milicu, mais sans bande sensible, avec les points du dessus raremeut cerclés de ferrugineux.

\& Un peu plus palle, ayant les points ment pour les ailes inféricures, dont les points noirs sont sourent obli. térés, surtout en dessus. II n'est pas très-commun, les femelles surtout sont difficiles id obtenir. 
noirs plus apparents, le disque du dessous des superieures roussatre et le fond de cette surface d'un brun plus jaunatre.

Necasus.

Bdv. var. Cassiope.

Epipmox. Knoch, Ochs. Treits. Janthe. IIub. 402? God. pl. 16 P. fig. $5-4$ ?

Nous ne l'avons pas ru. Il ne differe, dit-on, qu'en ce que les points noirs sont en totalité ou en partie effacés.

Les points des ailes supéricures sont Montagnes du Hartz, Les différences que généralement plus sentis, souvent pu-clairières des bois de nous signalons ici ont pillés de blanc, et le troisième est ali- sapin, dans les lieux été observées par M. né avec les autres au lieu d'être rejeté exposés au soleil. en arricre; les points des femelles sont presque toujours pupillés de blanc.

MELAMPUS,

Envergure, 5o mill.-Ailes d'un bruu

Prairies des Alpes Sa petite taille est son /Ochs, God. pl. 16 fig. 5-6.

Bdy. Icon. pl. 35 . 0 5-6.

oir; les supérieures arec une bande ferrugineuse, divisée par les nervures de la Suisse, de la Savoice, le la France, du tinc en taches presque rectangulaires, dont Tyrol, de la Carinthie trois ou quatre marquées d'un trés-petit etc. En juillet.

Janthe. Hub. 624-625. point noir, les inferieures arec une ban-

Le Montagnard. Engr. de semblable, mais dontles taches sont bien plus arrondies, surtout les anales, et marquées aussi de points noirs. Dessous d'un brun roux, avec les mêmes taches, mais plus claires et ordinairement plus incertaines, vu le moins d'intensité du fond, surtout vers le disque des supérieures.

ㅇ D'un brun moins foncé; plus ponctuée, arec le disque des supérieures plus roux et les inferieures grisatres en dessous.

Piante.

Un peu plus grand. Taches ferrugi- Mêmes ipoques Ochs. God. IIub, 491-neuses tout-ì-fait dépourvues de points localités. 494. noirs, celles des ailes supérieures étant Bdv. Icon. pl. 35. fig. encore plus rectangulaires que dans 7-8.

Melampus, celles des inférieures un Dup. Suppl. pl. 34. peu ovales, l'anale toujours plus petite fig. $1-2$. dividus du musée de Vienne et sur ceux de la collection de iI. Treitschke, qui en tenait un de Knoch et d'Ochs. Pour nous, on nous a communiqué quatre Epiphron; mais deux ne différaient point de Cassiope, ct les deux autres étaient des Melampus. Nous ne saurions donc regarder cette especc comme bien authentique, d'autant plus que M. Treitschke lui-même dit dans son supplément qu'elle n'est probablement qu'une variété locale de Cassiope, assertion que confirme encore sa rareté et la localité circonscrite dans laquelle on l'a trouvée jusqu'ici.

principal caractère distinctif, car il raric la bande ferrugineuse. Il aime a se poser sur les fleurs, et se laisse aborder et prendre assez facilement.
Hêmes mocurs que Melampis. Cette variété étant très-constante, il est possible qu'clle doive former une espèce; mais on ne pourra décider cette questinn que lorsqu'on connaitra les chenilles. et manquant quelquefois complétement. 
MNESTRA.

Hub. 540-5 45.

Bdv. Icon. pl. 55. fig. $1-4$.

Dup. Suppl. pl. 34. fig. $3-4$.

IIlaw.

Ochs. $3 \mathrm{dr}$.

PYRIHA.

Hub. 255-256, 616 . God. pl. 15. fig. 5-4. Sat. Machatie. God. Encycl. Le petit Nègre hongrois, Engr.

IIub. $213-284$

Bdv. Icon. pl. 35. fig. 5-6.

Dup. Suppl. pl. 48. fig. $1=2$.

Meisner.

(Trans. de la Soc. Hèlvét., an. $1827, p$. ;8.)

0 chs. God.

OEME.

Hub. 55o-555.

Bdv, Icon, pl, 54, fig. 5-8.
Envergure, 32 mill.-Ailes arron-| Prairies des monta-| On le rencontre dies, d'un brun noir; supéricurcs avec gnes de la Suisse et de dans quelques localités une bande ferrugineuse s'etendant sur la Savoic. En juillet. des alpes des cantons le disque du côte interne, coupée par les nervures en taches rectangulaires presque égales, dont la deuxième et la troisième souvent marquées chacune d'un point noir très petit; inférieures avec une bande semblable, mais plus courte et sans points noirs. Dessous des supérieures avec tout le disque ferrugineux et la côte et une bande marginale bien arretée, d'un brun clair teinté de roussâtre. Dessous des inférieures du même brun, arec un sentimentde bande anté. terminale.

o $D^{\prime}$ un brun plus clair, arec la bande plus pâle, visible en dessous et marquée de part et d'autre, aux supérieures, de deux gros points ordinairement oculés et sourent aux inféricures, en dessus seulement, de trois points semblables.

Ecosse.

Nous n'avons pas vu cette variété

Envergure, 40 mill. - Ailes d'un brun gonées), arec une bande ferrugineuse, Pyrénées, etc., etc. En pes de la Suisse. II formant aus supérieures destaches ova- juillet et aoot. n'habite pas a une les, dont les deux premières et quelquefois la quatrieme marquées d'un point noir, et aux inférieures de petites trches rondes, souvent marquées chacune d'un point noir. Dessous d'un brun. melé de ferrugineux arec les mêmes bandes, mais plus claires, surtout aux inférieures, où elles forment unc bande d'un fauve jaunâtre, large, souvent continue et plus prononcée il la crite, ces dernières ailes ayant souvent aussi $d$ la base deux ou trois taches ferrugineuses.

Plus pale, ayant le dessous des inférieures d'un brun jaunatre, avec la bande toujours continue et d'un jaune d'ocre, et la base du même ton et dessinant par en haut la ligne basilaire.

Differe de Pyrrha en ce qu'il est en-

Mêmes localités, grande élévation; son rol a quelques rapports avec celui de Blandina. Peu de Satyres nègres varient autant que lui ; les indiridus des Pyrénées, du Piémont et de l'Auvergne sont ordinairement moins : marqués de fauve et constituent sourent la variété suirante. un brun terne en dessus et mais particulièrement nement qu une variéte en dessous, sans aucune tache; quelque-Pyrénées, et surtout Au- de Pyrrha, avec lequel fois cependant on remarque quelques/rergne. petits traits fauves en dessous, it la place qu'occupent ordinairement les ban-

des, surtout dans la femelle.

Alpes de la Suisse.

variétés intermédiaires.

Envergure, 58 mill.-Ailes entières, Alpes de la France, 11 habite les mêmes un peu oblongues, d'un brun noir; les de la Suisse, du Tyrol localités que Pyrrha, supéricures marquées à l'angle apical et de la Savoie. Fin te mais il est moins réd'une tache ferrugincuse, géminée, por-juin et premiers jours pandu; il le précede et tant deux petits yeux noirs qui se répé-de juillet. dure peu de temps. tent toujours en dessous. Dessous d'un| |Nous possédons une ra- 
Dup. Suppl. pl. 34. fig.|brun un peu plus terne que le dessus, 5-8.

Hub. $5-8-5-9$.

Ochs. Bdr. God. pl. 16 P. fig. $1-2$. ayant ordinairement en dessus et en dessous quatre taches ferrugineuses rondes, marquées chacune d'un ocil noir dont le deuxième et le quatrième (en partant de la côte) plus grands.

o Plus pale, ayant les yeux bien plus marqués, surtout aux inférieures. Dessous plus jaunatre et un peu roux sur

Envergure, 40 mill.-Ailes d'un brun

taches ferrugineuses, petites, oblongues, uni, sans aucune bande; les inférieures le disque, aux supérieures. noirâtre, un peu plus intense et tris-four- de la Suissc et du Tyrol. ni de poils a la base, arec une série de En juillet. ct dont plusieurs aiguès du côté interne, et chargées chacune d'un petit ail noir pupillé de blane et au nombre ordinairement de six sur chaque aile. Dessous plus terne et ofirant les mêmes carac. tères; les inférieures sans bande sensibie.

Plus grande, plus pale, ayant les taches plus jaunes et les ailes inféricures légèrement polygonées.

MEDUSA. Fal, Ochs. Bdr. Hub. 203-204. God. pl. 15 o. fig. 5-6? Le Franconien. Engr.

IIippomedusa.

Psodea.

Ochs. God.

Hub. 497-499.

Bdv. Icon. pl. 3/. fig. 3-4.

Dup. Suppl. pl.40. fig. 1-2.

Eumenis. Dahl.
Envergure, 42 mill.- - iles entières, arrondies, d'un brun noir, avec uneban- Allemagne, Suisse, de maculaire d'un ferrugineux plus ou dans les bois élevés. En moins jaunatre, composée de taches mai et juin.

dont les premières, quatrième et sixième plus petites aux ailes supérieures, tendre, arec une bande quand elles y existent, et les deux der-dorsale d'un vert foncé nicres très-arrondieset très-isolées et dont liseréc de blanc et trois les deuxième, troisième et cinquième autres lignes latérales ves, et pour le ton de (quelquefois même la sixième) chargées du même vert dont leur couleur. N'ayant chacune d'un oil noir, dont ceux du l'inféricure également pas vu en nature l'indisommet rapprochés, mais non con-liscréc de blanc; tête et vidu figuré par Godart, fuents; infericures ayant trois ì quatre pattes rertes. Vit en nous ne sarons si c'est taches anté-terminales fauves, arron- avril et mai sur le $\mathrm{Pa}$ - une variété de Medusa dies, assez égales et chargées chacune nicum sanguinale. d'un ceil noir. Dessous d'un brun un peu moins intense, un peu teinté de roux et offrant les mêmes caractères que le dessus, seulement les yeux apicaux des su. péricures sont sourent seuls visibles et toujours séparis.

q D'un brun plus pâle et plus jaunatre, avec les taches fauves plus pâles et les yeux plus grands.

N'en differe que par une taille beaucoup plus petite.

Les taches faures sont plus grandes, presque contiguës; anx supérieures elles portent cinq yeux, dont les deux apicaux beaucoup plus grands, confluents et surmonlés sourent d'un sixieme plus petit; les inférieures ont également les yeux plus grands. Le dessous est un peu plus terne et les inféritures y sont marquées de six yeux.

f Plus pâle, plus jaunâtre, arec les taches faures plus grandes, plus pîles et les yeux plus grands. riété qui est entièrement dépourvuc d'yeux en dessus.
Cefte espèce n'est pas commune et ses lo-
calités sont assez restreintes en Suisse. Son vol est analogue a celui de Blandina. Elle aime it se poser sur les tleurs et se laisse approcher facilement.

Il n'est pas rare. On le trouve d'ordinaire. montagnes, cependant il descend quelquefois en plaine. Il varie beaucoup, surtout pour If nombre des taches fauou bien un Celo mate. Npes de la Styrie.
Styrie et IIongric. Ln juillet.

L'Eumenis de Dahl, que nous arons sous les yeux, n'est pas même variété du Psodea, dont les individus different seulement un peu entre eux par la taille et la vivacité des taches faures. Nous pensons que la connaissance de la chenille est indispensable pour séparer le $\boldsymbol{P}$ sodea du $\boldsymbol{M}$ edusa, 
Ochs. 13dr. Pyrene. II ub. 223-224.
God. pl. 14 N. fig. 1-3.

Envergure, 42 mill.-Ailes entièes, t'un Suisse, Tyrol, Piémont, le vol et les mocurs de avee une bande d'un ferrugineux foncé, Styrie, etc., etc. Fin de Pyrrha. Il présente une très-légèrement sinuée extérieurement, juin etcourant de juillet. assez grande quantité profondement denté interieurement, ce qui la fait paraître maculaire, surtout par en bas, chargée de trois yeux, dont les deux du sommet contigus et le dernier isolé, et souvent d'un ou deux autres plus petits, dont l'un apical et plus petit, l'autre intermédiaire et souvent sans pupille. Dessus des inférieures avec une bande maculaire chargée de trois at cinq yeux. Dessous des supérieures brun, ayant la bande ferrugineuse enliere, large, bien tranchee, et sur laquelle ne paraissent point les deux yeux accidentels du dessus. Dessous des inférieures du même brun, arec une bande anté-marginale un peu plus claire, à peine sensible, sur laquelle sont les yeux du dessus, mais plus petits et presque toujours sans iris ferrugineux. Antennes. brunes en dessus, blanches en dessous.

o Plus terne, arec les yeux accidentels plus marqués en dessus et ayant en dessous le sommet des supérieures et le fond des inférieures saupoudrés de gris, qui dessine souvent sous ces dernières la ligne médiane en l'éclairant de blanchâtre, et parfois, mais plus légèrement, la basilaire.

AIELAS.

Envergure, 47 mill. - Ailes un peu fig. $1-2$. Bdv. Icon. pl. 35. fig. 3-4.

Dup. Suppl. pl. 59 . fig. 1-4. dont certaines variétés| se confondent avec lui. de variétés surtout dans les femelles, qui sont tantôt semblables aux mâles et tantôt très-différentes. oblongues, noires; supéricures avec deux yeux apicaux contigus, très-pupillés de inférieures avec trois yeux pareils. Des-

Montagnes de la IIongrie, environs de Media. En juin et juillet. sous noir; les supéricures avec un sentiment de bande un peu plus claire et quelquefois légèrement ferrugineuse; les inféricures avec une bande anld-terminale à peine sensible et les yeux du dessis.

ㅇ D'un brun noir, arec les yeux apicaux des supérieures saupoudrés à l'entour de ferrugineux et un quatrième œil aux inférieures. Dessous des supérieures brun, à sommet cendrí, arec une large bande ferrugineuse bicn entière. Dessous des inferieures d'un gris cendré, strié de brun, arec la ligne médiane plus foncée et les yeux du dessus.
Il est encore rare dans les collections. Toutes les femelles que nous avons vues differaient extrêmement des males, ainsi qu'on peut le roir par notre description; cependant M. Boisduval dit qu'elles en different peu, ce qui fait penser que sa description a été faite sur une rariété.

Cette espèce est bien voisine de Stygne, dont elle differe surtout par l'absence du ferrugineux; mais on nous assure que le mâle a quelquefois un léger iris de cette couleur autour des yeux apicaux. Il serait done possible que le Melas fot une variété locale et très-constante de Stygne. 'Toutefois comme les auteurs modernes, et surtout M. Treitschke, qui en a vo une énorme quantité, persistent à les séparer, nous arons suivi leur 
LEFEBVRE!,

Bdv. Icon. pl. 53. Gg. $1-2$.

Dup. Suppl. pl. 55. fig. 3-4 et 39 . fig. 5-6.

Treits. Suppl.

Nelo. Hub. 105-106?

Alecto. God. pl. 14 N. fig. 5-6.
Envergure; 42 mill.-Ailes entières, d'un noir brun; les inférieures sans bande fauve, les supérieures en ayant quel- midi, cascades de Gaquefois des traces plus ou moins qenibles, avec des yeux semblables pour le gnoles. I la fin d nombre et la disposition a ccux de juillet.

Stygne, mais souvent plus grands. Dessous des inférieures d'un noir plus foncé et plus velouté qu'aux supérieures, mais sans aucune bande sensible. Intennes d'un noir brun en dessus et en dessous, avec le côté externe de la massue teinté de gris ou de roussatre.

of Plus pale, ayant toujours la bande fauve apparente, surtout en dessous, souvent les yeux plus grands et plus nombreux et quelquefois des traces de bande sous les inférieures. exemple. Le Melas de| Godart, que nous arons vu dans sa collection, est bien le véritable. Hautes - Pyrénées,
de Levitz et du coup au Melas, mais on l'en distinguera toujours facilement par les antennes et l'absence de bande sous les inféricures: dans les mâles. II est possible que le Nelo d'Hubner doive se rapporter au Melas: Quant a Alecto de Godart, il est d'autant plus probable qu'il se rapporte ici, qu'à son retour des. Pyrénées, en 1822 , M. Lefebyre, qui avait pris plusieurs Lefeborei, donna une grande quantité de doubles a Godart; l'un d'eux lui aura donc probablement servi de modele pour sa figure d'Alecto.

Le Lefebrrei est encore rare dans les collections.

ALEC'TO.

Ochs. Hub. 515-516. 528-529.

Bdv. Icon. pl. 32, fig. 4-7.

Dup. Suppl. pl. 33. fig. $1-4$.

Esp.

Pluro.

ARACHNL:

G'est à peine une variété. Il est seulement d'un ton plus foncé et sans apparence de bande.

Envergure, 45 mill,-Ailes entières, Enrergure, 49 mill.-Ailes un peu Alpes de la France,
- Alongues, d'un brun noir, tantôt sans de la Suisse et duTyrol. aucune tache, tantôt avec une légère En juin et noot.

éclaircie ferrugineuse près de l'angle apical; tantôt enfin, mais plus rarement, areo deux petits yeux apicaux a pein peu près du même ton que le dessus, avec un sentiment de bande plus claire ou légèrement ferrugineuse. Dessous des infericures d'un noir velouté, sans bande ni taches.

o Plus grande, plus claire, avec la bande fauve plus sênsible et se continuant sourent-sur les inférieures. Dessous des supéricures avec le disque plus ou moins rougeatre. Dessous des inférieures d'un brun terne, un peu sablé deblanchâtre, arec une bande terminale plus claire, mais peu sensible.

Fab. Bdr. Hub. 215un peu oblongues, d'un brun noir; lessu- etc., etc., dans les prai217.

périeures aree une bande ferrugineuse ries des montagnes. PreGod.pl. 16 r. fig. $7-8$.

Pronoe. Ochs.

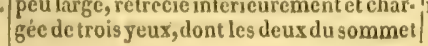
Pyrénées, Iongrie,
e., ete., dans les prai-
iére quinzaine d'aout. Sommet des alpes du

Il vole dans les endroits rocailleux les plus élevés des Iautes- $\Delta$ lpes, près des neiges éternelles, et n'est pas commun. Il est bien certain que celte espèce est quelquefois oculée, cur II. Lefehrre a $v u$ deux mâles qui offraient cette particularité; la figure d'IIubner n'est donc pas fautive, comme on l'a prétendu. Cette observation nous prouve de nouvcau que tous les Negrestendent at se rapprocher d'un typo commun, et c'est ce qui les rend si difficiles it étudier. M. Lefebvre s'est assuré chez H. Escher que les Papillons Glarialis et Tisiphone d'Esper sont bien des $A$ lecto. Quant à Godart, il n'a pas connu cette espèce. u canton de Berne.

Il est assez répandu, et varie suivant les localités. Dans les Pyrénées et en Allemagne, il s'écarte peu de celte des- 
contigus et plus grands; les inférieures avec trois yeux plus petits, placés chacun. sur une tache ronde ferrugineuse. Dessous des supérieures avec la bande plus large et marbre de gris rosed a l'angle apical. Dessous des inférieures sans yeux, d'un stis rosé, strié et ondé de de brun, avec deux bandes d'un brun rougedtre; la première médiane, large et sinueuse, la seconde terminale et plus étroite.

우 D'un brun plus pâle et jaunâtre, avec les yeux plus grands et l'inférieur suivi quelquefois d'un quatrieme plus petit. Dessous des supérieures ayant le disque ferrugineux, la bande plus jaunatre, le gris rosé remplacé par du gris jaunatre et les bandes brunes plus claires et non rougeatres.

P'iтno. llub. $574-577$.

BLANDINA.

Fab. Bdr. et 7 quint. fig. 3 . Ethiops. Id. Encycl. Medea. Ochs. Hub. 220222.

Le grand Nigre à bandes fauves. Engr.

NEORIDAS. Bdv.Index.Icon. pl. 29. fig. $1-4$. Dup, Suppl. pl. 36. fig. 5-6.

Treitsch. Suppl. gineusedes supérieures réduite à deux taches a l'angle apical et quelquefois tout-d-fait nulle. Yeux plus petits ot souvent non pupillés; inféricures sans yeux. Dessous d'un ton plus chaud.

† Analogue à la précédente.

Envergure, 44 mill.-Ailes d'un brun

lieu interieurement et exterieurement et imitant grossièrement une semelle, sur laquelle sont quatre yeux, dont les deux supérieurs plus gros et réunis, l'inféricur isolé, et l'intermédiaire très-petit, quelquefois nul, presque toujours sans prunelle et rejeté à. l'extérieur; inféricures légèrement dentées, avec trois ou quatre yeux sur autant de taches ferrugineuses. Dessous des supérieures plus clair que le dessus et mêlé de ferrugineux. Dessous des inférieures $d^{\prime \prime}$ เn rouge brun, arec deux bandes blanchâtres, dont l'une basilaire, l'autre antéterminale, plus apparente, sinuée, point ou peu denticulée en dedans, et sur laquelle sont les yeux du dessus, dont la pupille est seule bien apparente.

o Plus grande, plus claire, ayant la frange d'un blanc jaunâtre et le dessous des inférieures d'un ton jaunâtre ou verdâtre, les bandes plus blanches et plus prononcées, surtout celle de la base, et les yeux ordinairement plus apparents et s'élevant quelquefois jusqu'd cinq.

Envergure, 41 mill.- $-\Lambda$ iles tres- $€ n$ atteignant presque te bord interne, ayant
Ailes moins oblongues, bande ferrunoirâtre: supérieures plus obscures sur Allemagne, Suisse,

Alpes de la Suisse, ura, etc.

assez courte, arrondio, déprimée au mi- et aoot. Départements de l'I- Il a ù peu près les lieres, très-arrondies, d'un brun noirâ- sère, de la Drôme, de mœurs du précédent, tre, avec une bande d'un faupe ferru- la Lozère et des Basses- mais il préfere les mongineuse, large aux supérieures, surtout Alpes. Depuis la fin de tagnes séches, quoiqu'il par en haut, un peu aiguë par en bas et juin jusqu'it la mi-aont. n'habite jamais à une quelquefois une légère échancrure ì son milieu, mais seulement du côtè externe; ehargée d'yeux pareils pour le nombre (cription; en Suisse, au| contraire, il en est assez. diflérent et constitue presque toujours la variété suivante. Le bord marginal des quatre ailes en dessus est sourent. saupoudré de gris cendré.

C'est le plus commun des Satyres Nègres. I toute la plaine et surse poser sur les graminées et est facile à prendre. Il dure très longtemps, puisqu'on le trouve quelquefois jusqu'a la fin de septembre. Il varie peu; aussi n'a$t$-on point encore formé d'espèce à ses dépens. très-grande élévation. II n'est pas cncore trèsrépandu dans les collec. tions, sans doute parcel 
fet la disposition à ceux de Blandina, mais dont l'intermédiaire manque presque toujours. Inférieures marquées de trois yeux sur des taches ferrugineuses. Dessous des supéricures d'un brun un peu rougeatre et marqué de gris cendré à l'angle apical. Dessous des infétrieures d'un brun grisatre terne, arec une bande anté-terminale plus claire, médiocrement marquée, légèrement violitue, fortement dentée en dedans, un peu fondue extérieurement et sans yeux.

+ Plus petite, ayant les bandes fauves plus claires, le disque des supérieures roussâtre en dessous, et les inférieures d'un gris-verdâtre pâle, avec la bande blanchâtre.

\section{NERINE.}

Treits.

Bdr. pl. 51. fig. 6-7.
Dup. Suppl. pl. 34 fig. 5-6.

Escher.
Envergure, 44 mill. - Ailes entières, d'un brun noir; les supérieures un peu aigués au sommet et ayant une bande . d'un ferrugineux foncé, légèrement maculaire, un peu rétrécie inférieurernent, et marquée de deux yeux contigus; les inférieures arec une bande semblable, plus maculaire, formant quatre taches, dont les trois postérieures marquées chacune d'un cil. Dessous des supérieures $d^{\prime}$ un ferrugineux foncd, avec la bande plus claire, plus large qu'en dessus et coupée netternent, el presque droil par le bord terminal, qui est brun. Dessous des inférieures d'un brun noir jusqu'd la ligne médiane, qui est dentée intéricurement el suivie de quelques atomes blanchatres, puis un peu plus clair, avec les yeux du dessus, mais sans bande faure. Antennes grises en dessous.

을 les ailes supérieures plus arrondies, les inférieures dentées, les quatre avec la frange entrecoupée, d'un brun beaucoup plus clair et plus jaunâtre que le male, les bandes fauves moins rouges, moins maculaires; les yeux du sommet plus grands, réunis et accompagnés ordinairement d'un troisieme plus bas. Dessous des supérieures d'un faure jaunatre à sommet gris; dessous des inféricures gris jusqu'à la ligne médiane, qui est coupce bien nel, puis blanchâtre, avec deux d cinq yeux bien marqués. Ligne anté-terminale bien visible, continuc et exactement parallèle aux dentelures du bord terminal.

Plus grand (50 mill.), plus arrondi; bande ferrugineuse plus large, plus vive, micux tranchée, et chargée de quatre dans la plaine. In juille yeux plus grands. Dessous des supérieu- et nonf.

res ayant la bordure marginale brune plus large et plus foncée; dessous des inférieures d'un brun plus foncé et noirûtre, arec les lignes moins distinctes. Antennes brunes en dessous.

+ Un peu plus petite, d'un brun plus pále, avec les bandes d'un fauve jaunitire, et offrant quelques atomes grisatres sous les inféricures.
Suisse, canton des. Grisons et du 'Tessin, et
Alpes de la Carinthie, Hongrie?
septembre.

aodit $c$

Il est fort rare et encore peu répandu dan les collections. Nous. avons longuement décrit la femelle, parce qu'elle est encore peu connue, surtout en France. Flle differe autant du male que celle de Melas. Le mâle, au contraire, pourrait être confondu avec celui de $\mathbf{S}$ cipio, mais on l'en distinguera par le dessous des inférieures, ou les yeux du dessus se répètent, et dont la ligne médiane est dentée et suivie d'atomes blanchâtres. Les femelles se distingueront facilement par la ligne médiane du dessous des inférieures, qui n'existe point d'une manière sensible chez $\dot{S}$ cipio. Le Nerine varic en ce qu'on roit quelquefois un quatrieme oil, mais plus petit, sur la bande faure des inférieures. qu'on l'aura confondu
avec Blandina, dont notre description le fera facilement distinguer.
Nous n'ayons point vuce Satyre, et les différences que nous donnons ici nous ont été communiquées par $\mathbf{M}$. Bugnion, qui les a fait également insérer dans les Annales de la Société Entomologique. Depuis il a trouvé la femelle, qui, nous dit-il, differe de son mâle comme Goantc femelle diflere du sien. 
SCIPIO.
Bdv. Icon. pl. 3o. fig. Les supérieures un peu aiguës au som$1-6$.

Dup. Suppl. pl. 38. fig. 5-6.

\section{DROMLS.} clopídie.

Cleo. IIub. 209-213.

God, pl. 17. fig. 5-6. Tyndarus, Ochs.

Corcodronus.

Variété plus petite, plus foncée, ayant Lefeb. Collect. Nobis. à peine quelques traces de la bande ferrugineuse et complétement dépourvue d'yeux. Dessous plus foncé.

q Semblable.

me oil et trois aux infericures, dessous de ces dernières $d^{\prime}$ un gris cendre uni, sans aucune ligne, avec un à quatre points noirs sans pupille. Inféricure légerement dentées.

Envergure, 35 mill. - Ailes arron-

neux foncé, sur laquelle on voit aux stpérieures deux yeux apicaux contigus. Inférieures tantôt sans yeux, tantôt avec trois ì quatre placés sur des taches d'un ferrugineux foncé. Dessous des supéricurcs d'un rouge brun, avec la côte et le bord d'un gris cendré, une lunule fermant la cellule, puis un trait, bruns, et les deux yeux du dessus. Dessous des inférieures d'un cendré plus ou moins blanchatre, avec une large bande médiane denticulé des deux cótés et plus foncée sur les bords, puis une termiarrêtée. P'alpes et dessous des antennes $d^{3}$ un gris blanc.

o Ayant la bande fauve plus pâle et les yeux plus gros. Dessous plus pale; supérieures avec les traits bruns plus prononeés, formant quelquefois une bande plus foncée sur ses bords et renfermant la lunule discoildale; inférieures plus pûles.
Sa description est trop] courte pour que nous puissions juger si le Styx constituc une espèce distincte du Nerine, ou seulement une variété locale.

Département des Bas.

Ce Satyre rarie en ce ses-Alpes et de l'Isére? que les ailes inféricures du mille sont quelquefois marquées de trois. ycux, mais plus petits qu’aux supérieures, el en ce que celles-ci ont quelquefois, au lieu du troisième œil, un petit point noir sans pupille. La femelle nous a été communiquéce par $\mathrm{H}$. Lefebvre, qui la posséde depuis fort long-lemps et croit l'atroir prise aux environs de Grenoble. Le Scipio est encort très-rare dans les collections. dies, d'un brun-noirâtre très-chatoyant se, Italic, Styrie, etc. au vert, avec une bande d'un ferrugi- En juin et juillet. nale de la même couleur, mais moins

est commun et varie i l'infini, tant pour les yeux que pour la taille et l'intensité des couleurs. La figure de Godart a été faite sur. des individus pris dans les Pyrénées et trèsoculés; dans les montagnes plus froides les yeux deviennent plus petits, et leur pupille disparaît; enfin ils s'effacent quelquefois complétement, comme dans la variété ci-dessous. On distinguera toujours sarement tous ces indiridus des espèces voisines par leur petite taille, la couleur grise du dessous des inférieures, ctc.

Le Dromus labite les plus haules montagnes, mais jamais à une trèsgrande élévalion; son rol est rapide, mais il se pose souvent par terre ou sur les pierres.

Mont Talifie.

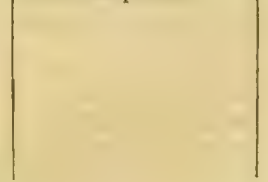


Esp.

Neleus. Freyer.

MANTO.

Fab. Ochs, lidr.

Hub. 5 12-514.

Var. accid. $207-208$.

God. pl. $17.6 \mathrm{fg} .7-8$.

Le grand Negre bernois. Engr.

ARETE

II ub. $25 z-232$.

Ochs, Goul. Bdv.

STIRIUS.

God. Encyc!

Parmanio. Bneb. Bulv.

GORGk.

Ochs. Bdv.

IIub. 502-505.

God.pl. 34 \%. fig. 3-4?
Plus grand; bande fauve du dessus Hongrie, Dalmatic,

plus clairc, plus vive et plus large; yeux Autriche, etc.

apicaux tout-at-fait coniluents. Dessous

d'un gris mêlé de jaune.

$q$ Encore plus claire et à bande fauve très-jaunatre.

Envergure, 40 mill. - Ailes arrondies, un peu oblongues, d'un brun terne, avec une bande d'un ferrugineux noirâtre, peu arrêtée sur ses bords et chargée de points noirs non ocelles, au nombre de quatre aux supéricures, et le plus souvent de trois aux inférieures. Disque des premières un peu roussátre en approchant de la bande, et souvent marqué de traits noirs dans la cellule. Dessous des inférieures d'un gris brunâtre, arec les lignes basilaire et médiane brunes, sinuées et formant une bande qui se rétrécit au bord abdominal. Palpes d'un brun noiratre. Antennes it massue ferrugineuse en dessous.

† Plus terne, plus pâle en dessous, arec les lignes des inférieures mieux marquées.

Envergure, 53 mill.-Ailes entières, d'un brun noiratre; supéricures un peu anguleuses au sommet, arec une bande ferrugineuse, continue, marquée de trois points noirs, dont les deux apicaux oculés; inféricures avec cinq points oculés, placés tous, cxcepté l'anal, chacun sur une tache ferrugineuse. Dessous des premières ailes d'un roux clair, avec la côte verdâtre et la trace des yeux apicaux. Dessous des inférieures entièrement d'un gris verdâtre, arec les points du dessus marqués en blanc et ombrés intérieurement de noir.

Malgré toutes nos rccherches, nous n'arons pu parvenir at nous procurer cette espéce, qui n'a jamais été figurée. En voici une description d'après Godart. Envergure, 2 pouces environ.-Ailes d'un noir brun, avec une bande ferrugineuse chargée aux supérieures de deux yeux dont l'antérieur double, aux inférieures de quatre. Dessous des supérieures ferrugineux, arec les bords cendrés. Dessous des inférieures entièrement cendré, avec une bande anté-terminale plus claire et sinuée, sur laquelle sont quatre yeux correspondants at ceux du dessus.

Envergure, $5 \eta$ mill. - Ailes un peu anguleuses, d'un brun noir'; supérieures du Tyrol, Pyrénesarec une bande ferrugineuse foncée, juillet et anot.

Russic, Styrie.

Alpes autrichiennes. ce Satyre que par la figure d'Hubner, qui a été faite sur un individu femelle unique que possède le Musée impérial de Vienne. Aussi son excessive rareté ne nous a-t-elle pas permis de juger s'il constitue une espèce distincte.

L'individn femelle unique sur lequel Godart a fait sa description, appartient \& M. Dejean. Dans son Index M. Boisduval assurc qu'il n'est qu'une variété du Parmunio de Bober: Cependant il est surprehant que cet entomologiste n'ait donné dans son liones ni l'un ni l'autre de ces Satyres si peu connus.

Il n'est pas très-com. mun. Il habite le sommet des montagnes, et préfére les endroits rocailleux. Son rol est plus rapide que celui de ses congénères. Il se distinguera des suivants 


\section{G. SATYRUS.}

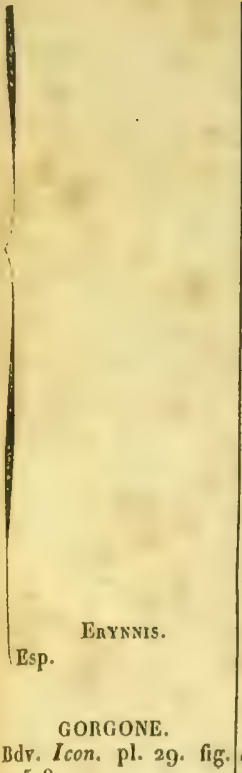

5-8.

\section{GOANTE.}

Ochs. Bdr.

God, pl, 17 Q. fig. 3-4. Scaa. Hub. 233-234. ment très-petits, rapprochés, mais sé-| parés, et rarement d'un troisième; inférieures avec la bande ferrugineuse plus arrêtée intérieurement et souvent chargée d̀ son extrémilé marginale de trois petits yeux. Dessous des supéricures ferrugineux, avec la bande un peu plus claire, denticulée extérieurement, la côte et le bord marginal d'un brun légerement strié de gris. Dessous des inférieures d'un brun noir, strić de gris, avec les trois lignes plus foncées, découpées en dents aiguës, la médiane et l'anté-terminale laissant entre elles une bande plus claire.

q Plus pále, arec la frange légèrement entrecoupéc, la bande ferrugineuse des supérieures plus pâle, s'étendant un peu sur le disque par en haut, et dont le bord intéricur est plus denticulé; dessous plus pâle; celui des inféricures plus clair, plus jaunatre, avec les lignes plus visibles et quelquefois les yeux du dessus.

N'en différe que parce qu'il est dépourvu d'yeux en dessus et en dessous.

Envergure, 36 mill. - Ailes enticres, g. arrondies, d'un brun noir plus foncé sur le disque, avec une bande maculaire d'un rouge brun fonce se confondant presque avee la couleur du fond, chargée aux supéricures de trois yeux, dont les deux apicaux contirus mais non réunis, le troisième plus éloigné et quclquefois nul, et aux inféricures de trois yeux et souvent d'un point noir antérieur. Dessous des supérieures ayant le disque d'un forrugineux foncé, avec la côte et le bord terminal d'un brun noiratre légèrement strié de blanchatre, et les yeux du dessus. Dessous des inféricures de cette dernière couleur, arec la ligne médiane et l'anté-marginale seules apparentes, laissant entre elles une bande plus claire surmontant les yeux du dessus.

ㅇ De la même taille, $d$ ailes plus oblongues; plus claire, avec la baude, d'un ferrugincux jaunâtre, plus large non maculaire, plus détachée du fond et portant quatre à cinq yeux. Dessous d'un gris jaundtre, avec le disque des supérieures ferrugincux, et aux inféricures les trois lignes visibles; les deux postérieures laissant entre elles une bande plus claire et très-nettement marquée.

Envergure, 42 mill. - Ailes brunes, sans reflet bien vif; supérieures avec Suises du midi de une bande continue d'un fauve-ferrugi-l Piémont, etc. En juillet. neux vif, presque droite interieurement, dentée extérieurement et chargée de trois yeux assez grands, dont deux apicaux souvent réunis, et un autre éloigné par sa taille et le ton foncé du dessous des inférieures. La figure de Godart est très-mauvaise et en donne une fausse idée. Peut-être se rapporte-t-clle à Gorgone.

Pyrénécs. En juillet.

Cette variété ressemble un peu, en dessus, au Mnestra.

Il existait dans beaucoup de collections, confondu avec Gorge et Goante, dont M. Boisduval l'a séparé avec raison. Il s'en distingue au premier coup d'œil par sa taille supérieure au premier, inféricure au. second, son reflet vert bien vif, le ton de sa bande ferrugineuse, et par les ailes de sa femelle, qui sont oblongues, non dentées, ni à frange entrecoupée. Nous arons vu une variété mâle dont les yeux aux supéricures étaient séparés par un point noir.

Le ferrugineux de ce Satyre est d'un ton plus ou moins chaud suivant les localités, mais il n'atteint jamais l'intensité de celui de Gorgone. Le nombre des/ 
plus petit. Inférieures avec la même bande chargée de trois à quatre yeux. Dessous des supérieures d'un fauve ferrugineux, plus foncé jusqu'à la bande, avec la cóte et le bord marginal d'un brun clair strié de blanchâtre. Dessous des inféricures de ces dernières couleurs, avec les trois lignes, dont la médiane plus distincte et plus bordée de blanchâtre, et trois ot quatre petits yeux.

오 Plus claire, ayant quelquefois un petit œil au-dessus des dcux apicaux. Dessous beaucoup plus marqué de blanchâtre, avee les nerzures des inférieures de celte couleur. Inférieures dentées, frange légèrement entrecoupée.

EVIAS.

Envergure, 45 mill. - Ailes entieres, Linndenne.

God. Bdv. Icon. pl. 31 fig. 3-5.

Dup. Suppl. pl, 37. fig. 1-2.

Treitsch. Suppl.

Bonellii. IIub. $892-895$.

rrondies, brunes, avec une bande d'un alpes de la Suisse. En fauve ferrugineux; supérieures ayant juillet.

cette bande large, mais assez courte, continue, arrondie inféricurement, marquée de cinq yeux noirs, dont les deuxiéme et troisieme plus gros, réunis, confluents, placés un peu obliquement, le quatrième et le cinquième isolés; inféricures avec la bande plus étroite, parfois maculaire et marquée de quatro à six yeux. Dessous des supérieures semblable au dessus. Dessous des inférieures d'un brun foncé velouté, légèrement strić de blanchâtre, avec une bande anté-marginale sinueuse, plus claire, plus striée de blane, et sur laquelle se voient plus ou moins les yeux du dessus.

o Plus pâle, ayant le disque lavé de ferrugineux en dessous, et les inférienres plus, pâles, surtout à la basc.

EPISTYGNE.

Envergure, 43 mill.-Diles entières, Département du Var Bdv. Index Icon. pl.51. arrondies, d'un brun noirâtre; supé- et des Basses-Alpes. fir. 1-2.

IIub. $855-858$ et Stygne. $639-640$. ricures avec une éclaircie dans la cellule Italie. En mars.

Dup. Suppl. pl. $3 \eta$. fig. 3-6. ct une bande ante-terminale d'un jaune d'ocre pale; celte dernière large, longue, déchiquetée intéricurement, rétrécic par en bas et chargée de cing à six yeux dont les trois supérieurs conlluents, les deuxième et troisieme plus gros, placés très-obliquement; inférieures avec une bande un peu maculaire, $d^{\prime}$ un roux obscur et marquées de cinq à six yeux. Dessous des supérieures ferrugineux, arec une tache costale et le bord interne noirs, et les yeux du dessus. Dessous des inféricurs d'un brun clair très-strié de blanchâtre, avec les nervures de celte couleur, une large bande médiane plus foncée, et les yeux très-petits et à peine risibles.

\& Plus pâle, arec la bande jaune plus large, micux arrêtée inféricurement, yeux des ailes supéricures s'ẻlère quclquefois jusqu'd cinq ou six et est une nouvelle preuve de cette observation, déjì bien connue, que le nombre des yeux ne saurait être invoqué comme un caractere bien constant dans les Satyres nègres. LC GQante n'est pas fort commun; il habite les prés, le bord des chemins pierreux et des torrents; il a le vol et les moeurs de Blandina La figure de Godart est fort bonne.

On pourrait décrire le dessous des ailes inférieures de cette espèce (ainsi que de plusieurs analogues) en disant qu'elles sont d'un brun pâle, strié de blanc, arec une bande médiane large, sinueuse, plus foncée, surtout sur ses bords, et une autre terminale plus ćtroite; ce qui serait exact, surtout pour la femelle. Mais ta base de l'aile dans los mâles est quelquefois si chargée de brun, que la partie interne de la bande médiane y devient fort per distincte. Nous avons donc préféré considérer le brun foncé comme le fond de la conleur. L'Evias n'est pas commun.

Il n'est pas encore très-répandu dans les collections. Son yol est lourd et il selaisse facilement. approcher. Il dure très-peu de jours et n'a qu'une seule gínération par an. Il est sujet à graisser, surtout les femelles. 
|plus pâle aux inféricures, les yeux plus grands, l'inférieur souvent bipupillé et le dessous des secondes ailes plus clair.

AFILA.

Fab. God. Bdv. \begin{tabular}{r|r} 
Envergure, 40 mill.-Niles entières, & Russie méridionale, \\
assez targes, d'un brun noirátre, avec & Dalmatie belle espèce,
\end{tabular} (ranchée, se rap-

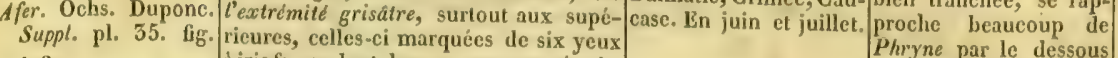
$1-2$. a iris faure dont deux au sommet réunis,

Phegea. Ilub. 500- rentrant en dedans, et surmontés d'un 501. $749-751$.

autre plus petit et très-rejeté extéricurement; les srois autres égaux, alignés, et dont le dernier quel quefois double; inféricures marquées aussi de six yeux. Dessous des supérieures ayant le disque légèrement ferrugineux. Dessous des inférieures brun, avec les nervures blanchâtres, deux traits de cette couleur dans la cellule et sept à huit yeux entourés de gris ou de fauve et dont les extrêmes souvent sans pupille.

f Plus grande, avec l'extrémité des ailes d'un gris plus jaunâtre et les yeux plus grands.

Dalmata.

God. Encycl.

périeures sont contigus, à l'exception Afra. Bdv. Icon. pl. de l'apical, et dont l'inferieur manque 34. fig. 1-2? en dessous. des inféricures; elle paraît varier fort peu, du moins tous les individus que nous arons vus sont-ils très-semblables. Elle est jusqu'ici trèsrare dans les collections.

Dalmatie.

(108) Frange des quatre ailes entrecoupée de gris et de noir dans les deux sexes.

EURYALE. Ochs. Bdv. Hub. $789-790$. God. pl. 13. fig. 3-4.

Phlomera. IIub. $218-219$.

ADYTL Hub. $759-760$.
Envergure, 42 mill.-Ailes d'un brun| Alpes, pyrénées, etc., 11 n'est pas rare et noir arec une bande ferrugineuse; cel-|etc. En juillet et aoot. chargée de trois à quatre points noirs, petils, ordinairement non oculés; celle des inférieures chargée aussi de trois d quatre variant de taille et plus souvent oculés; ces dernières dentées. Dessous d'un brun très-mélangé de ferrugineux, surtout sur le disque des supéricures, ou la bande est toujours plus clairc. Dessous des inférieures avec une bande anté-marginale d'un gris brun plus clair que le fond, sur laquelle se roient les ycux du dessus, plus petits, souvent cerclés de ferrugineux et dont l'anal plus gros.

+ Plus pále en dessus et plus souvent oculéc. Dessous des inferieures d'un brun-verdâtre pâle, avec la bande blanche ou blanchitre, beaucoup micux tranchée du côté de la ligne médiane où elle est très-dentée, surtout vis-à-vis de la cellule.

Très-légère variété femelle qui a les yeux un peu plus grands, ceux du soinmet toujours conflents et un quatrième œil intermédiaire sur les supérieures, oculé ou non.

Ordinairement plus petit qu'Euryale (53 a 35 mill.). Ailes supéricures un peu plus arrondies el marquées des mêmes points, mais plus gros et constamment les prairies des montagnes. Il varie prodide ses variétés se rapprochent du Ligea ; mais il est constamment plus petit, et le mále n'a point de bande blanche en dessous. Les individus d'Allemagne different is quelques égards de ceux qu'on trouve en France. Les points noirs manquent quelquefois complétement. Nous signalons cette espéce comme une des plus importantes à étudier parmi les Satyres nègres. gieusement, et plusieurs 
foculés aux quatre ailes. Les deux de| l'angle apical réunis.

LIGEA.

Lin. Fab. Ochs. Bdr.

Hub. $225-227$.

God. pl. 13. fig. 1-2.

Envergure, 48 mill.-Ailes d'un brun

IIongrie, forêts du Il est commun. Ses noir, avee une bande ferrugineuse bien nord et de l'est de la mœurs sont celles du tranchée aux quatre ailes, continue, si- France, etc., etc. En Blandina. Il habite de nuéc des deux cótés et un peu rétrécic juillet et aoat. préférence les bois siLe grand Nigre hon- au milieu sur les supérieures, ou elle Chenille épaisse, tués au pied des mongrols. Engr.

est marquée de quatre points noirs courte, d'un gris-jaunâ- tagnes, mais il descend trés-gros, ocules, mais a pupille petite, tre clair, avec unc lignc aussi dans la plaine; et bien visible seulement sur les deux d'un brun foncé sur le c'est un des plus grands supérieurs, qui sont aplatis, contigus et vaisseau dorsal, bordée de cette division. souvent réunis; chargée aux inférieures de deux lignes plus claide trois yeux égaux, bien pupillés; ces res, puis deux autres mêmes ailes. dentées, ayant le dessous lignes de ce dernier ton, d'un brun roussâtre, avec une bante mé- sous la dernière desdiane blanche, étrnite, irrégulière, inter-quelles sont les stigmarompue, partant de la côte, où seule-tes, qui sont nettement ment elle est bien visible et se perdant vers marqués en noir; tête le milieu de l'ailé, et les yeux du dessus. et pattes de la couleur of Plus terne, avec les yeux mieux du fond. Se troure en pupilles, encore plus visibles, surtout septembre, puis en mars en dessous, et souvent accompagnés et avril, sur les gramiaux supérieures d'un point noir placé nées.

sous le dernier oil. Bande blanche des Chrysalide renflée, inféricures se répétant du côté de la li-d'un brun jaunâtre, gne basilaire. ponctuie de noir, areo plusieurs traits de cette couleur. Se trouve cachée entre les herbes et non suspenduc.

EMBLA.

Thunberg. Nobis.

Ethus, Fab.

Dioxippe. Hubn, 538539 .

Dup. Suppl. pl. 56. fig. $i=2$.

DISA

Thunberg. Nobis.

Griela. Fab. God. Hub. 228-229.

Stheno. Ilub. 561 -502 ,

Embla, Ochs.

Bdv, Icon, pl, 32, fig. 1-3.

Dup. Suppl. pl. 3\%. fig. 3-4.
Envergure, 44 mill.-Diles d'un brun roussâtre; les supérieures légèrement Torneo, Dalécarlic. $\Lambda$ saupoudrées de gris au sommet, surtout la fin de juin.

en dessous, et chargées de part et d'au-

tre de quatre points noirs a iris d'un fauve jaunatre, dont les deux supéricurs beaucoup plus gros, réunis, a iris commun, tris-rentrants en dedans, toujours oculés, et les deux autres plus petits, souvent sans pupille; inférieures légèrement polygonees, avec trois a quatre points noirs $a$ iris faure et quelquefois oculés. Dessous des inférieures brun, saupoudré de gris au bord terminal et a lignes ordinairement non visibles, mais indiquées par deux ou trois taches d'atomes blanes, plus serrés et plus apparents a celle du bout de la cellule; des points noirs correspondant aux yeux du dessus.

Plus terne, avec les yeux plus grands, un peu plus égaux, di iris coniluents et accompagnés d'un cinquieme au bord interne des supérieures.

Envergure, 44 mill. - Ailes entieres d'un brun terne; les supérieures sau-
poudrées de gris au sommet et marquées d'une bande continue, courbe, d'un ferrugineux clair, qui est chargée de quatre points noirs isolés, rarement ocellés, et dont les deux premiers trèspeu rentrants et presque alignés, surtout en dessous; inlérieures sans aucune tache. Dessous des supéricures différant|
Laponic, environs de

Laponie méridionale.

En juillet.

Cette espéce et la suirante ont été confondues par presque tous les auteurs, sans doute à cause de leur grande rareté, et leur synonymic était fort embrouilléc. Nous arons vu les deux sexes de l'un et de l'autre, et nous pensons, arec M. Duponchel, qu'elles doirent former deux espèces distinctes; sculement, il cst facheux que cet auteur u'ait pas figuré les deux sexes, qui sont fort différents, ainsi qu'on peut en juger par notre description. Nous avons da restituer à ces Satyres les noms que Thunberg, qui les a bien décrits, leur a imposés le premier.

Cette espèce n'est pas moins rare que la précédente, mais elle est plus répandue qu'elle dans les collections; où clle porte a tort le nom d'Émbla. Elle offre quelques variétés, mais peu tranchées. Tel est le Stheno d'Hubuer, qui a 
du dessus en ce que la bande fauve est maculaire et forme un iris à charque œil. Dessous des inféricures brun, trés-saupoudré de blanc et paraissant cendré, avec les trois lignes bien visibles, la ba. silaire et la médiane formant entre elles une bande large, dentée, plus foncée, bordée extéricurement de cendré plus blanchatre que le fond, surtout près de la côte, et l'anté-terminale isolée, étroite et netlement détache des deux côtés.

q Plus terne, plus roussâtre, a bande ferrugineuse plus pâle et comme salie, avec les points plus gros et légèrement locellés.

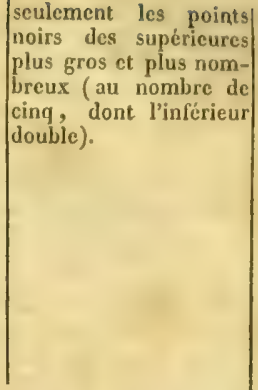

(109) Chenille glabre. - Nervure cosiale tress-renfle d la base, la mediane seulcment un peu dilatie. -Antenncs greles, à massue pyriforme. Ailes arrondies, d'un brun noir uni dans les mâles. - Taille au-dessus de la moyenne.

\section{Les Ericicoles. Dup. *}

PILEDA.

Lin. Ochs, Bdv.

Hub. 127-129.

God. pl. 7 quart. fig. 2 Le grand Negre des bois. Engr.
CORDULA,

Fab. Treits, Bdy.

God. Encyel.

Ochs. Ilub. 149-150. Var. $q .619-620$.

Bryce. Ochs. Hub. $724-727$.

God. pl. 12 x. fig. 5-4 Ferula. Fab.
Envergure, 55 mill.-Ailes d'un brunnoir chatoyant en violet; supérieures arrondies, entières, arec deux yeux noirs séparés par deux points blancs; inféricures polygonées ou légèrement dentées, arec un petit œil près de l'angle anal. Dessous des supéricures avec l'ocil apical plus grand et cerclé de jaune. Dessous des inféricures brun, strié de brun plus foncé, arec les lignes médiane et anté-terminale plus foncées, bordées extérieurement de stries blanchâtres, et le plus souvent deux points noirs entre elles, prés de l'angle anal.

$q$ D'un brun jaunatrc, arec une bande faure plus ou moins marquéc, mais formant toujours, au moins aux supéricures, un iris à chacun des ycux; inféricu-
Il est communet aime d se poser sur la bruyère. Quelquefois un des deux yeux des supérieures est accompagné d'un tre et cerclés de jaunatre en dessous; cendré ou roussâtre pâ-autre plus petit. Telle inferieures avec un seul, beaucoup plus le, avec une ligne dor- est la femelle figurée plus 作 brunes, pattes écailleu- environs d'Auxerre, et ec ses brunes. Vit en juin dans laquelle les ailes lus sur l'Avena elatior. du cóté gauche sont traChrysalide arrondie, versées de part et d'autre d'un fauve clair, cott-parunelarge bandeblanchée à terre entre les che; tandis que, du côté herbes. droit, elles sont comme dans les indiridus ordinaires. Engramelle a aussi figuré celte singulière varjété.

Alpes, Piémont, Cette belle espece Suisse, Espagne, etc. n'est pa's très-rare; nous En juin et juillet. l'arons vue voler fréquemment dans les montagnes de la Catalogne. Presque tous les auteurs en ont fait deux especes; cela vient de ce qu'on rencontre quelquefois des mâles arec un peu de jaune sous les ailes supérieures. Le nombre des yeux varie et s'élère parfois jusqu'd quatre, au moyen de ce que les points blancs intermédiaires sont cernés de noir;

- M. Boisduval, en partageant le genre Satyrus en neuf races (Icones, p. 199), u'a fait aucune mention des trois espèces qui composcnt cette dirision. Nious ignorons si c'est uu simple oubli ou s'il a voulu créer avec elles un nouveau geare; cette dernière supposition nous semble peu probable. 


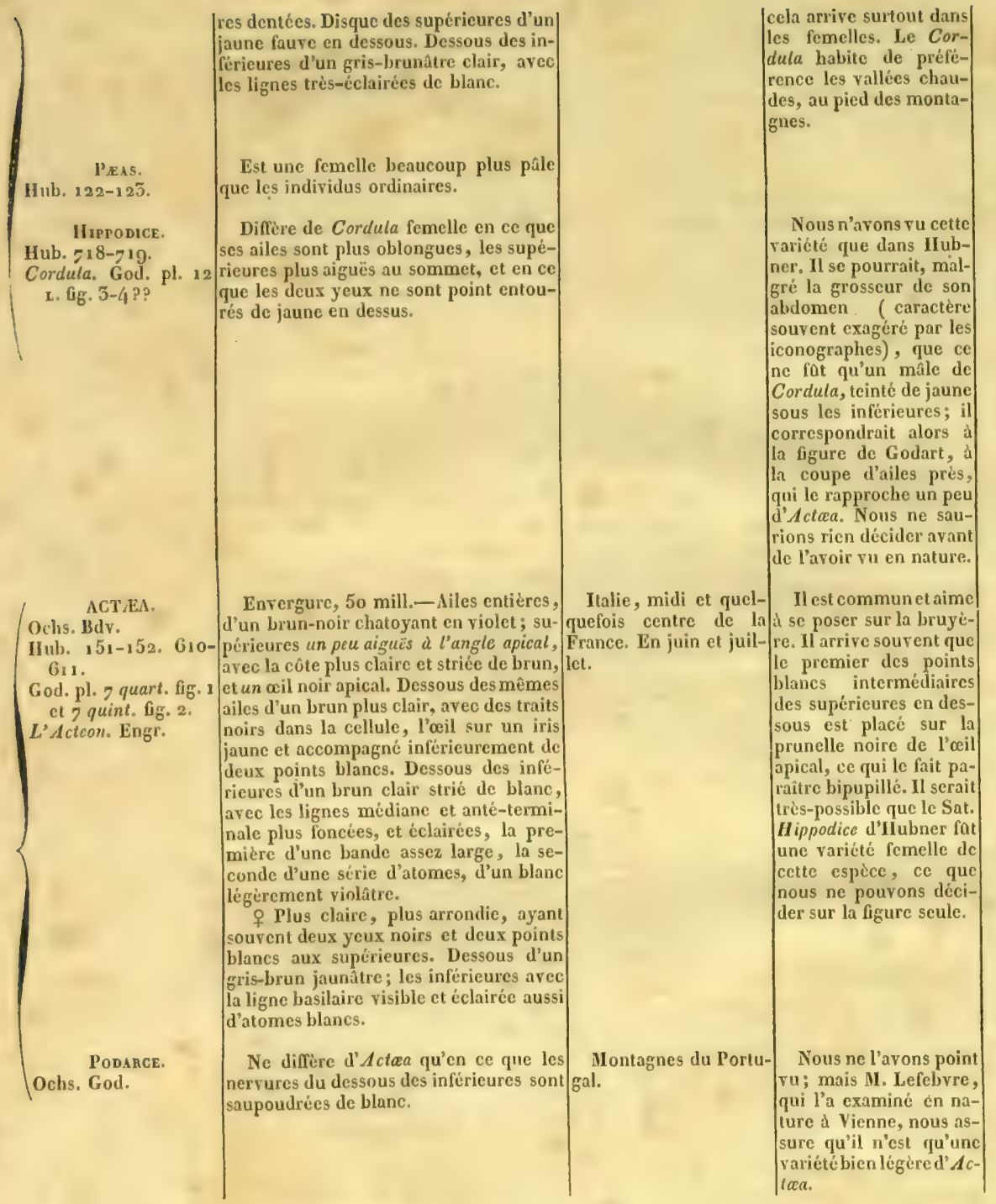

(110) Chenille glabre ou pubescente. - Nervures costale et midiane également tres-renfies d la base. - Couleurs varièes. - Taille mojenne et au-dessus.

(11) $\{$ Antennes à massue en bouton, distincte de la tige. . . . . . . . . . . . . . (112) Antennes d massue grossissant insensiblement et confonduc arec la tige. . . . . . . . (120) 
(112) Antennes d massue en bouton, distincle de la tige.

Les Rucipoles. Dup.

$(1,5)$

Ailes d'un brun-cendré foncé, sans bandes blanches ni fauves; supérieures ayant deux yeux noirs, séparís

ordinairement par deux points blancs, au moins en dessous. . . . . . . . . . . . . . (114)

Ailes d'un brun fonce, traversees par une bande d'un blanc jaunitrc ou faufe. . . . . . . . . . . (185

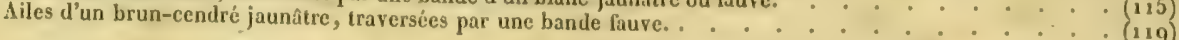

(114) Aites d'un brun-cendre foncé, sans bandes blanches ni fauves; supéricures ayant deux yeux noirs, séparcis ordinaircment par deux points blancs, au moins en dessous.

Fab. Bdv.

God. pl. 7 tert. fig.

et 7 quint, fig. 1.

IIub. $507-509$.

Statilinus. Ochsenhei

Treits.

Le Coronis et le Faune. Engr.

Envergure, 46 mill. - Ailes d'un brun

Dans une grande par-| 11 n'est pas rarc. On cendré; supérieures plus foncées et plus tie de l'Europe. En aod. voit souvent sur les invelues sur le disque, arec deux gros points noirâtres anté-marginaux, quelquefois pupillés de blane, surtout l'antérieur, et séparés par deux petits points blancs; inferieures avec la ligne antéterminale un peu plus foncee et un point noir près de l'angle anal. Dessous d'un gris cendré, plus foncé jusqqu'au milicu; les supérieures arec les yeux du dessus cerclés de jaune; les inférieures avec la moitić postérieure nébulcuse, et une bande d'un cendré blanchatre longeant la ligne médiane. Frange des quatre ailes d'un gris sale.

o $\Lambda$ peine aussi grande, ayant une large bande anté-terminale d'un jaunc d'ocre saipoudré de brun.

Allionia 0chs. Treits. Ilub. 818-819 et Fauna, 510-511. L'Arachne. Engr.

AUTONOE.

Fab, God, Treits.

Hub. $157-138$.

Bdy. Icon. pl. 41. fig. 5-6.

Dup. Suppl. pl. 28 . fig. $3-4$.

L'Icare. Engr.
Plus grand, plus foncé en dessus, Italic, mididela Franbeaucoup plus obscur en dessous, ou le ce, Hongrie, etc., etc. deuxième oeil des supérieures est ordinairement sans iris jaune; lignes ou traits de l'intérieur de la cellule plus marquées. Inféricures très-foncées en dessous, avec la ligne basilaire toujours bien marquée.

Envergure, 53 mill.-Ailes d'un brun cendré, avec la frange trés-blametén de la Russio

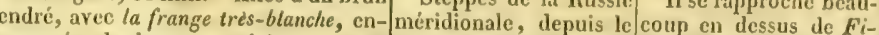
trecoupéc de brun; supérieures arec Don jusqu'au Caucase? dia. On roit sourent, une large bande anté-terminale plus bords du Volga, Cri-entie les yeux des supéclaire, sinuẻe, nettement coupée inté- mée? En juillet. rieurement, fondue extéricurement et portant deux yeux noirs ćcartés; inférieures avec la même bande, légèrement striée, marquée près de l'angle anal d'un petit œil, et suivie d'une ligne anté-terminale interrompue, plus foncéc. Bande du dessous des supérieures lavée de jaune d'ocre. Dessous des inférieures d'un brun clair tres-strié de blanc, avec les nervures blanches, les trois lignes plus foncées et éclairées de blanc, surtout ln médiane, qui est suivie d'un rang de très-petits points blanes.

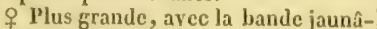
tre et s'étendant sur le disque des supéricures. férieures une série de points blanchûtres audessus de la ligne antéterminale. Il habite les endroits arides et converts de loruyères, vole peu, et se pose de préférence sur les pierres en cachant ses ailes supéricures avec ses inféricures.

La connaissance de la chenille nous parait indispensable pour l'ériger en espìce séparéc. points blancs comme dans Faune; mais lo mále que nous arons vu. en était totalement dépourvu. On observe éralement sur le disque des supérieures un épi relu et noiratre, mais plus large et plus foncé que dans Fidia. Autonoc est fort rare dans les collections, quoique commun, à ce qu'il parait, dans los localités qu'il habite. L'individu décrit par Ochsenheimex n'est qu'une varićté de Scmele. 
FIDIA.

Jin. Fab. Ochs, Bdv. Hub. $147-148$. God, pl. 11 K. fig. 3-4. Le Faune. Engr.

Envergure, 5 z mill.-Ailes d'un brun Italie, Portugal, FranIl est tris-commun| cendré, ayant la frange très-blanche, en-. ce méridionale, etc. En dans les endroits secs et trecoupee de brun, arec une apparenee juillet.

de bande anté-terminale, large, et distincte par des places plus claires de son côté interne, bordée de son côté externe par une ligne ondulcuse noiratre; supérieures ayant cette bande chargée de deux gros points noirs faiblement pupillés de blanc et séparés par deux gros points blanes; inférieures ayant un petit point noir près de l'angle anal et parfois une série de petits points blancs. Dessous des supérieures gris, avec des taches blanchatres et les yeux cerelés de jaune. Dessous des inférieures varié de gris et de blanchâtre, avec les trois lignes marqquées en noir; la médiane formant un angle très-saillant vis- $i-v i s$ l'extrémité de la cellule.

o Plus grande et plus arrondic.

pierreux des environs de Montpellier, où nous l'arons pris abondamment. Il a les mours de Fauna.

(15) Ailes d'un brun fonce, traxersées par une bande d'un blanc jaunâtre ou fauve.

(116) \{ Bande transverse d'un blane plus ou moins jaunatre. . . . . . . . . . . (117)

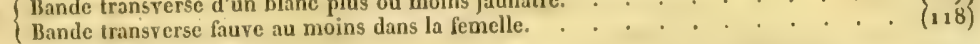

(117) Bande transverse d'un blanc plus ou moins jaunâtre.

BRISEIS.
Lin. Fab. Ochs. Bdr. Hub. 150-151. God. pl. 7 . fig. 1. L'Hermite. Engr.

\section{Pinata.}

Esp. Hub. 604-605.

Ochs. God.

ANTHE.

Bdr. pl. 40. fig. 3-4.

Dup. Suppl. pl. 27. fig. 5-4́.

Persephone. Hub. 589590. 710-711.
Envergure, 5o mill. - Ailes brunes, Dans une grande par-| Il est commun dans arec une bande transverse d'un blane tie de l'Europe. En juil- les endroits pierreux jaunâtre; supérjeures axec la cóte jaunâ-let et aoot.

tre, le disque velu, et la bande divisée

par les nerrures et marquée de deux yeux noirs; inféricures a bande continue et fondue extéricurcment, arec un petit oil près de l'angle anal. Dessous d'un jaune d'ocre très-pâle, arec les bandes continues et des taches brunes; inférieures avec les lignes médiane et basilaire laissant entre elles une bande brune interrompue dans la cellule, et formant ainsi deux grosses taches. Antennes d'un gris blanchâtre.

ㅇ Beaucoup plus grande, arec les bandes plus larges, mieux arrêtées, et les inferieures n'ayant pas les deux taches noires près de la base, mais seulement les lignes plus obscures.

Variété femelle plus grande, d'un ton plus chaud, arec la bande d'un jaune Bohême. En juillet et d'ocre foncé de part et d'autre. Dessous aout.

des inférieures d'un gris plus cendré.

Envergure, 65 mill. - Ailes brunes, Russic méridionale, avec une bande d'un blane jaunatre et Syrie. En juillet.

la frange entrecoupée; supérieures cen-

drées à la base, arec la bande divisée en taches par les nervures. l'apicale joignant la cote en se recourbant intéricurcment, et marquée d'un gros point noir non pupille, la suivante très-petite ou nulle, et la quatrieme marquéc pareillement d'un gros point noir, non pupillé; inféricures et arides. En Dalmatic et en Turquic les individus sont plus grands que dans le centre de l'Europe et atteignent presque la taille de Circe.

Nous l'arons souvent pris sur les rochers des montagnes, a Castelnau, près Montpellier.

Il est encore tres-rare dans les collections. Les individus sur lesquels nous faisons notre description ont été pris sur le mont Liban, le 21 juillet. Ce Satyre offre aussi, comme Briseis, une variété dont les bandes sont d'un jaune 


\section{G. SATYRUS.}

dentées, avee la bande continue comme

dans Briseis. Dessous des supéricures

variẻ de gris et de jaunâtre strié, avec

les yeux pareillement areugles. Dessous

des inféricures gris, strié de brun, avec

les neroures delachées en blanchâtre.

ㅇ Semblable.

CIRCE.

Fab. Bdv.

Envergure, 72 mill. - - Ailes d'un brun

noir, aree une bande transverse blanche, méridionagne, France

méridionale, etc., etc. le mill common blane: supericures ayant la bande divi- aoot. séc par les nervures en taches dont l'a- Chenille rase, épaisse, Il aime également d̀ se picale marquée d'un gros point noir, luisante, d'un gris livi-poser sur le trone des parfois oculé, les troisieme et quatrième de, striée par places de arbres cariés. Nous l'apyriformes ou aiguës extérieurement; in- roussâtre, avec trois vons souvent pris ainsi féricures dentées, arec la bande conti- bandes d'un noir verda- aux environs de Mont-

nue. Dessous des supérieures avec la tre, dont une dorsale pellier.

même bande, l'œil toujours pupillé, et plus foncée, et, au-desdeux taches blanches dans la cellule. Des- sus des pattes, une bansous des inféricures strié de gris blanc de jaunâtre sur laquelle et de brun, avec la bande du dessus, et sont placées les stigmade plus, la ligne basilaire très-éclairée de tes, qui sont noirs. Tête blanc. Massue des antennes noire, al rousse, rayée de noir; sommité fuute.

ventre et pattes d'un

o Plus grande, ayant ordinairement gris - rougeâtre livide. it la côte des supérieures une tache blan- Vit en mai sur plusieurs che qui va rejoindre intérieurement la graminées, et se cache tache qui porte l'œil, et quelquefois un sous les pierres pendant second ocil sur la quatrième.

le jour.

Chrysalide arrondie, d'un brun rougeâtre, avec les stigmates, et surtout ceux du cou, grands et saillants. Dans une petite cavité, sous la terre et sans être attachée.

HERMIONE.

Lin. Fab, Ochs. Bdr. Hub. 122-124. God. pl. 7 sec. fig. 2. Le Syloandre. Engr.

Ochs

Atcrose.

Hub. 125-126.

Envergure, 70 mill.-Ailes d'un brun Dans une grande pararec une bande transyerse un tie de l'Europe, bois une grande partic de la blanc enfumé, et la frange entrecoupie; secset pierreux. En juil- France. Il a les mour: supérieures ayant la bande presque con-let et aoot.

tinue, à peine interrompuc par les ner- Chenille rase, rilée vures, très-saupoudrée d'atomes bruns, transversalement, d'un surtout vers le haut, où elle est chargée gris roussâtre, arec un d'un ooil brun; inférieures dentées, avec double filet dorsal brun la bande continue et un petit oeil près et une bande latérale de l'angle anal. Dessous des supérieures d'un gris cendré borayant la bande teintée de jaune, l'œeil noir dée d'une ligne noire et distinct et le sommet strié de blanc. liserée de blanc. 'Tête Dessous des inféricures brun, strié de d'un jaune d'ocre, rayée gris, arec les trois lignes marquées en de noir. Vit en mai sur noir et l'oil anal. Massue des antennes plusieurs graminées. toute noire.

Chrysalide et mours

f Plus grande, avec la bande des su- semblables a celles de périeures moins obscure et souvent Circe.

marquée de deux yeux noirs.

Plus petit (6o mill.).-Bande des in- Allemagne, DalmaDup. Suppl. pl. 27. éloignée du bord; bande des supéricu- En juillet ct aoat. fig. 1-2.

Bdv. Icon. pl. 40, fig. $5-6$.

res marquée, souvent dans le mâle et

Dessous des inférieures plus marqué de Hermione, var, God. noir.

Le petit Sylucund. Engr.

les collines pierreuses.

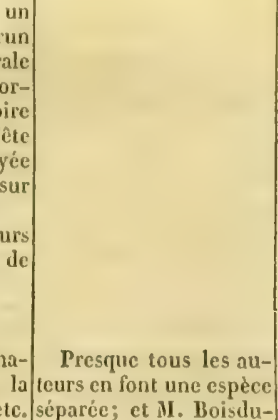

du précédent. ochracé, La figure 589ce cas.

$$
\begin{aligned}
& \text { Il cst commun dans } \\
& \text { ne grande partic de la } \\
& \text { rance. Il a les mours }
\end{aligned}
$$
ral, tout en hésitant is suivre leur exemple, annonce qu'il donnera sa chenille incessamment. Nous attendrons qu'elle ait été fidèlement com- 
(118) Bande transverse fauve, au moins dans la fomelle.

NEOMIRIS.

God. Encjcl. Hist. pl. 11 K. fig. $1-2$.

Bdr. Icon, pl. 42 , fig. 6-8.

Marmor 817 .
Envergure, 50 mill. - Ailes d'un brun noir, avec la frange entrecoupée et une sur les hautes montabande fauve transrerse; supéricures gnes En juillet.

ayant cette bando maculaire inférieurement, très-saupoudrée de brun qui la cache en partie supérieurement, et marquée d'un œil noir apical et quelquefois d'un point noir sur la partie faure; inférieures un peu dentées, ayant la bande fauve large, plus claire intérieurement et marquee auprès de l'angle anal d'ur point noir. Dessous des supérieures arec la bande faure nette, large et seulement un peu teintée de brun par en haut, ou l'oeil apical est renfermé entre deux iraits blanchâtres. Dessous des inféricures brun, strié de plus foncé, arec une bande blanche nettement coupée par la ligne médiane, fondue de l'autre côté l'œil du dessus et une série de petits points blanes peu marqués.

ㅇ Avec la bande faure nette aux supérieures, où elle est toujours marquéc de deux yeux. Dessous des inférieures plus clair.

ANTIIELEA

Lefeb. BdY. Icon. pl. 41. fig. $1-4$. o Hub. 861-862.

Dup. Suppl. pl. 27 fig. 5-6.

o Telephass pap. exot.

Dup. Suppl. pl. 28. fig. 1-2.

THELEPHASSA. Hub. pap. exot.
Envergure, 50 mill, - Liles brunes, avec une large bande irrégulière, blanla frange entrecoupée; les supérieures entieres, avec un large trait noir longitudinal dans la cellule, et la bande ré. trécie au milieu et marquée de deux gros yeux noirs écartés, quelquefois sans pupille; iniérieures dentées, avec la bande interrompue avant le bord abdominul, plus large en cet endroit et y portant un petit oeil sur la partie rousse. Dessous des inférieures d'un brun strié, avec la ligne médiane bien marquée et éclairée par un large espace blane correspondant à la bande du dessus.

o Plus grande, plus arrondic, ayant les bandes du dessus fauves; celle des supéricures se prolongeant sur le disque, et deux points blancs entre les yeux. Dessous des supérieures fauve au milieu. Dessous des inférieures d'un gris mêlé de jaunâtre et strié de brun, sans espace blanc, et arec les lignes peu marquees.

Ailes un peu plus claires, bande du dessus entierement fauve et quelquefois
Ce Satyre a des rapports tris-marqués avec le précédent, et nous ignorons pourquoi les auteurs modernes l'en ont tant éloigné. Il n'est pas commun dans les collections, à cause des localités assez circonscrites qu'il habite. Il vole ordinairement sur les montagnes à 5 ou 600 toises d'élévation, mais il descend aussi quelquefois en plaine. La chenille a été découverte par M. Rambur, mais n'u pas encore été publiée.

Nora. Plusicurs auteurs citent comme synonyme de cette especc un Sat. Iolaus d'Ilubner. Ce Satyre n'existe point dans cet auteur; mais le Neomiris y est très-bien figuré sous le nom de Marmora, du nom de celui' qui l'a trouvé le premier.
Midi de la Hongrie? En juia.
Les deux sexes de ce Satyre sont si différents, qu'Hubner en arait fait deux especes; mais M. Lefelorre les a pris, le 12 juin, accouplés aux euvirons de Smyrne, où il n'existait point de mále a hande lauve, ce qui lève lout doute à cet égard. La véritable patric de ce Satyre est l'Asie-Mlineure, et il. n'est pas bien sar qu'il ait été pris en llongric; cependant, comme cette localité est indiquée par plusicurs auteurs, nous arons $=\mathrm{da}$. l'admettre dans nos tableaux. II est rare dans les collec. lions.

Nous n'arons pas vu len nature cette variélć. 
aussi deux points blanes entre les yeux des supéricures.

I. Boisduval observe qu'on devrait peut-être tha regarder comme l'espèce typique.

(19) Ailes d'un brun-cendré jaunâtre, tracerses par une bancie faute.

AHETHUSA.

Fab. Ochs, Bdv. Ilub. 154-155, 95

$9^{58 .}$
God. pl. 7 tert. fig. 2. la bange entrecoupće; supérieures avec
la bande formant cing ou six taches bien Le petit $A$ greste. Engr. nettement sciparies, et dont la premiere marquée $u^{\prime} u n$ gros point noir ; inférieures ayant la bande d'un fauve plus vif, formant quatre à einq taches ovalesoblongues, excepté l'anale, qui est ronde et marquée d'un point noir. Dessous des supéricures d'un jaune d'ocre, strié de gris à la cóte et au sommet, avec l'œil du dessus pupillé. Dessous des inféricures d'un gris brun très-strié de brun plus foncé, avec les lignes assez confuses, la médiane courbe, legerement sinueuse et éclairée de gris blanchatre.

o Un peu plus grande, plus claire, arec la bande faure plus large, moins maculaire et souvent marquée aux supéricures d'un second point noir. Dessous plus jaunitre.

ERYTII. II ub. $59^{1-59^{2}}$. Lin. Fab. Ochs. Bdv. Hub. 143-144. Var. accid. 82 $832-855$. God. pl. 7 tert. fig. 1. L'Agreste. Engr.

D'un ton généralement plus chaud. fort petites et arrondies; quelquesunes des supérieures marquées de petits points noirs: Disque des supérieures d'un jaune vif en dessous. Dessous des inférieures plus foncé, arec les trois lignes nettes, distinctes, fortement ombrées de brun foncé, la médiane et la basilaire formant cntre elles une bande brune, l'anté-terminale surmontée d'une séric de petits points blancs, comme dans Neomiris.

Envergure, 50 mill. - Ailes d'un

\section{large, presque insensible et chargée de} Bande transverse réduite à des taches Italie. Dans toutc l'Europe, brun-jaunatre clair, avec la frange en- dans les bois secs et ro-
trecoupée; supérieures aiguës au som- cailleux. En juillet et trecoupée; supérieures aiguës au som- caille
met et ayant une bande anté-terminale aoat. Chenil Ghenille d'un brun On rencontre parfois des deux yeux bruns écartés et lérerement cendrí joundividus plus grands et éclairés de jaune; inférieures dentées, cing. lignes plus ou sont d'une taille démearec la même bande, mais plus dis-moins marquées, dont surée. Nous arons au tincte et marquée près du bord terminal une rougeâtre latérale, contraire trouvé un inde quatre tackes d'un jaune d'ocre, dont la et une autre liserée de dividu chez lequel ces dernière chargée d'un oil noir. Dessous blanc, qui porte les stig- taches sont très-petites des supérieures d'un jaune d'ocre, plus mates; tête pâle, avec et sans pupille.

foncé a la base, arec les yeux du des-trois lignes brunes; sus écrits en noir. Dessous des inférieu- pattes roussátres. Vit en res d'un gris cendré trís-strié de brun, avril et mai sur les graaveo les trois lignes, dont la médiane minées.

distincte, très-sinueuse et éclairée d'une Chrysalide courte, large bande blanche. epaisse, non suspen-

o Plus grande, avec la bande des su-due, roussître, arec périeures bien marquée en jaune d'ocre, l'enreloppe des ailes et les ycux noirs. Bande blanclue du des- plus claire et le dos forsous des inférieures moins apparente. tronc des arbres cariés. bite. Ses moeurs different peu de celles; de ses analogues. Toutetois, il se prend, non-seulement sur les rochers, mais encore dans les bois ombragés. Les femelles sont moins communes que les mâles. Chez celles-ci l'œil apical est sourent pupillé en dessus.

Noтa. Plusieurs aurtcurs rapportent ici la var. Aristceus Bonelli, mais elle appartient évidemment à l'espèce suivante.

Il est commun et aime à se poser sur le

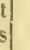
s. 
Aarstixs.

Bonelli.
Plus grand et plus vivement coloré.

o Plus grande, avec les bandes bien netles, d'un faure très-vif; celle des supérieures envahissant en outre unc partic du disque; celle des inférieures entièrement faure, et n'oflrant point, par conséquent, de taches de cette couleur. Yeux noirs et bien marqués. Dessins du dessous très-prononcés.

HIPPOLYTE

Ochs. Bdv. Icon, pl. 42.

Envergure, 50 mill. - Ailes un peu fig. 1-2.

Dup. Suppl. pl. 28. fig. 5-6.

Agaxe. Hub. 159-1/10. dicyone. Fab. God L'Hippolyle. Engr. oblongues, brunes, arec une bande d'un fauve jaunatre, liserée de noir, dentéc intéricurcment, marquée aux supérieures de deux gros points noirs écartés, et dont l'antérieur pupillé en dessous, ct aux inférieures d'un très-petit point près de l'angle anal. Dessous des inférieures d'un gris brun strié, avec les nervures blanchâtres, les lignes peu marquées; la médiane un peu éclairćc de blanchâtre à la côte; l'anté-terminale composée d'une suite de cherrons peu marequés, et lc point du dessưs accompagné d'un autre plus petit.

ㅇ Inconnue.
Corse, Sicile, Sar-l Le faure domine teldaigne. MI. Rambur a Iement chez la femelle élevé la chenille, et il de cette belle variété, ssure qu'elle ne différe qu'on pourrait dire arec point de celle de Semele. justessic qu'il forme le fond de la couleur.

Russie méridionale.

Cette espèce est exIrêmement rare. Nous ne l'avons pas vue en nature, et nous la décrirons sur les figures et description de M. Boisdural, qu'il assure être très-exactes et fitites surle seul individu existant en France et appartenant a M. Chardiny, de Lyon.

(120) Antennes d massue grossissunt insensiblement ct confondue arec la tige.

(Les IHerbicoles. Dup.)

NARICA

Hub. $704-707$.

Bdv. Icon. pl. 42, Gig. 3-5.

Dup. Suppl. pl. 29. fig. i-3.

EUDORA.

Fab. Ochs. Bdv. IIub. $165-36$ 亿.

God. pl. $18 \mathrm{n}$. fig. $1-5$. Le Misis. Engr.
Envergure, 42 mill.-Miles supérieu-| Russie méridionale, 11 est aussi extrêmeres entieres, faures, avec la côte, le bord entre le Volga et les ment rare. Toutefois interne et 1 bord marginal bruns, et monts Ourals. ayant sur le disque une bande oblongue noiratre, partant du bord interne et s'avançant obliq̨uement jusqqu'au bout inférieur de la cellule, puis ì l'angle apical un oil noir, aveugle en dessus, pupillé en dessous; inférieures légèrement dentées, brunes, sans taclies. Dessous des supéricures privé de la bande noire du dessus. Dessous des inféricures d'un gris brun sablé de noirâtre, arec les nerrures plus claires, les trois lignes sensibles, la basilaire et la médiane formant entre elles une bande plus foncée el ćclaircie Ie blanc dés deux cốés, l'anté-terminale sinucuse et un peu éclairée de blanc extéricurement.

$\$$ yant les ailes supérieures dépourvues de la bande noire discoïdale, et marquées près du bord interne d'un second point noir plus petit et non visible en dessous.

Envergure, 42 mill.-Ailes d'un brun Allemagne, Suisse, jaunâtre; supérieures aiguës au som-midi de la l'rance, etc. met, arec le disque velu et marqué d'un Én juillet et aoot.

épi grisâtre sans poil, et un point noir Chenille pubescente, recugle à l'angle apical; inféricures den- verte, avec une large tées, d'un brun uni. Dessous des supé-lligne latéralé, variée delsupérieures et la taille l'assertion de M. Boisduval, qu’il n'cxiste en France que les exemplaires de M. Chardiny et ceux de M. Franck, est dénuće de fondement, puisque nous avons fait notre description sur un mâle appartenant i M. Lefebvre. II n'est pas exact non plus de dire que cette espèce figure sur les catalogues des bords du Volga et de l'Oural. Nousn'arons pas vu la femelle *

II est très-commun et a les mêmes mœurs que Janira. Les femelles varient pour la largeur de la bande fauve des est asiatique, puisqu'elle 
Ochs. Bdiv.

God. pl. 7 sec. fig. 1.

Janir $a$ o ct Jurtina ơ Lin.

Fab.

Jurtina. Hub. 161162 .

Lin.

TITHONIUS,

God. pl. 7. fig. 2.

Tithonus. Ochs. Bdr.

Pilosella. Fal..

Ilerse, Hub. , 56-15 612 .

L'Amerillis, lingr.

Esp. Illig.

II ub. $59^{3}-596$. lo Ja inia.

Le Mirtil. Engr.

ricures d'un jaune d'ocre encadré de jaune et de ferrugineux, des yeux, surtout de gris, avec l'œil pupillé. Dessous des in-au-dessus de laquelle l'inférieur, qui s'obliférieures cendré, légèrement strié, avec est une ligne blanche; tère parfois. Nous avons les lignes à peine sensibles. Massue des tête verte, arec un trait un mâle au contraire, où antennes $d^{3}$ un fauce clair en dessous. roux bordé de blane; ce second oeil parait sur

q Plus pctile, ayant le disque des su- pattes vertes; pointes une bande légérement périeures d'un jaune d'ocre, avec la caudales ferrugineuses. fauve. La taille de ce base plus ou moins grisatre, jusqu'à une Vit en mai et juin sur Satyre varie aussi et ligne médiane plus foncée, et formani plusieurs graminées. égale parfois celle du ainsi une bande jaune marquée de deux Chrysalide suspen-suirant. Il a d'ailleurs yeux noirs.

due, entièrement verte comme lui sa variété ou d'un brun roux, arec Iispulla.

des lignes longitudina-

les blanchatres.

Envergure, 46 mill.-Ailes brunes; Dans toute l'Europe, Il est extrêmement supérieures entières, arec un épi velu et bòis, prés, etc., etc. En commun, La femelle al plus foncé sur le disque, et un oeil api- juin et juillet. cal à iris fauve, souvent suivi de quel- Chenille verte, un inférieures teintéc de ques taches de cette couleur; inférieu- peu velue, avec une li- fauve, mais jamais aures dentées, d'un brun uni. Dessous des gne plus foncée sur le tant que la variété suisupérieures d'un jaune fauve encadré de vaisseau dorsal, et deux vante. Engramelle figure gris jaunâtre, avec l'oil du dessus. Des- autres latérales blan- une foule de rariétés acsous des inférieures d'un gris jaunâtre, chatres, têtc et pattes cidentelles. Godart rapplus foncé jusqu'd la ligne médiane, qui vertes; pointes caudales porte à cette espéce l'Eest suivie de un a trois points noirs cerclés mêlées de roussâtre. Vit rymanthea d'Ésper; ne de jaune, et dont l'anal, quand il sur les graminées, en l'ayant jamais vu, nous existe, plus petit. Massue des antennes arril et mai. ne pourons dire si c'est d'un roux obscur en dessous. $\quad$ Chrysalide suspen-ici ou a l'Eudora qu'il o Plus grande, ayant aux supéricu- duc, d'unvertpâle, avec faut le ropporter, ou res une bande anté-terminale fauve, qui destubercules jaunâtres même s'il ne constitue s'étend plus ou moins sur le disque, et sur le dos et deux lignes pas une espéce distincte. sur laquelle est l'ail apical souvent gé-brunes sur l'enveloppe miné, et aux inférieures une bande anté- des ailes. terminale un peu plus claire que le fond. Dessous des inférieures plus clair.

Plus grand. Dessous des inférieure plus jaunâtre et marqué ordinairement de-quatre points.

o Plus grande, ayant la bande des inféricures entièrement fauve, ainsi que le disque des supérieures. Dessous des inférieures parfois un peu violâtre, et lavó de jaune près de la ligne médiane.

Envergure, 37 mill.- - Ailes faures,

Midi de la France, On rencontre parfois Espagne, Portugal, Si- cette rariété aux envicilc, etc. rons de Paris, mais elle n'y cst jamais bien tranchée. encadrées de brun; supérieures ayant tie de l'Europe, dans les milliers dans les bois de sur le disque une tache oblongue, velue, bois. En juillet et aoat. nos environs. Il préfére moins foncée sur ses bords et partant Chenille courerte de ceux qui sont secs et du bord interne, et un œil noir apical petits poilscourts et bi-couverts de bruyères, - bipupillé ; inférieures obscures a la base fides, verte ou roussa-mais il s'accommode et légérement dentées. Dessous des su- tre, arec une ligne dor- également des autres et péricures sans tache discoidale. Dessous sale rousse, puis une la- même des chemins bordes inféricures d'un gris roussâtre, arec térale semblable, puis dés de haies. Le mâle a la. ligne médiane éclairée d'une bande une autre au-dessus des souvent, et la femelle d'un jaune d'ocre, sur laquellesont pla- pattes, accolée a une presque toujours, un cés trois a quatre points blanes largement raie d'un blane jaunâ- petit oil près de l'angle cerclés de roussâtre, et dont les deux su- tre; tête rousse, rayée anal des secondes ailes périeurs isolés.

de brunatre; pattes et en dessus.

o Plus grande, d'un fauve plus clair, pointes caudales de la et dépourvue de tache noire discoïdale couleur du corps. Vit sur les supéricures. graminées.

Chrysalide suspendue, grisitre ou rougeattre, arec des lignes et dues points brunâtres. 
IDA.

Fab. Ochs. Bdv.

I ub. 158-r5y.

God. pl. 18 R. fig. 4-5.

PASIPHAE.

Ochs. Bdv.

IIub. $167-15 g$.

Bathseba. Fab.

God. pl, 18 k. fig. 6- $\%$,

Salome. Fab.

Le Tilire. Engr.
Envergure, 54 mill.-Ailes arrondies, Midi de l'Europe, Il n'est pas rare dans | fauves, encadrées de brun ; supérieures bois montueux. En juin. les garigues de Montayant sur le disque une tache brune par- Chenille couverte de pellicr, où nous l'avons tant du bord interne, coupde carrement poils courts et bifides, pris en quantité.

au sommet et interrompue par les ner- d'un gris roussâtre, avec vures, et un œil apical noir bipupillé. une ligne dorsale noiraDessous des supéricures dépourvu de tre, puis une bande plus la tache discoìdale. Dessous dés infé- claire marquée de six rieures d'un gris-brun nébuleux, avec ou sept points noirs, la ligne médiane coudée au tiers, éclai- puis au-dessus des patrée de gris satiné et d'une tache jaunc tes une ligne blanchaau bout de la cellule; base des mêmes tre bordéc d'une ligne ailes nuancée du même gris; ligne anté- rousse; tête grise, rayée terminale peu sensible et perdue dans de noir et de blanchaun espace d'un brun foncé : le tout sans tre; pointes anales de yeux.

Plus cetto dernibe couleur; - Plus grande, plus claire, et de-pattes de la couleur da pourvue de tache discoidale sur les su-fond. périeures. sur les graminées, et
surtout sur le. Triticum cespitosim.

Chrysalide suspen-

due, courte, épaisse,

d'un brun jaunatre, avec des points et des stries noirîntres.

Envergure, 42 mill. - Ailes arronEspagne, France midies, d'un jaune faure, encadrées de ridionale, sur les monbrun; supérieures' ayant depuis la base tagnes boisées. En juin jusqu'au milieu une large place brune et juillet.

légèrement coupée par les nervures, et i l'angle apical un oeil noir ordinairement bipupillé; inférieures un peu dentées, avec la base obscure et une série de petits yeux noirs, dont le deuxieme plus petit et souvent nul. Dessous des inferieures d'un brun clair, avec la ligne médiane suivie d'une bande d'un jaune clair, et surmontant une série d'ycux noirs a iris fauve, dont les deux supéricurs séparés des autres par une tache blanchâtre.

우 Plus grande, d'un fauve plus clair, avec lc disque des supéricurs non coupé par une tache brune et seulement un peu teinté de grisatre.
Il est fort commun dans les environs de Montpellier, où nous l'arons pris abondamment volant avec Ida. Cher la femelle, l'oil apical est quelquefois accompagné d'un point noir.

(121) Antennes très-distinctement annelées de blanc dans toute leur longueur en dessus et en dessous. . . .

(122)

( Chenille pubescente. - Nervure costale très-renfléc à son origine, la médiane également rẹnflée, quoique moins

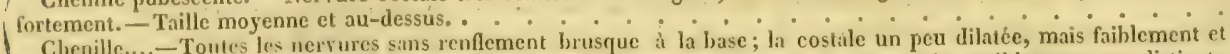
Chenille....-Tontes les nervures sims renflement brusque a la base; la costile un peu dilate, mas faiblement et (122) longuement; lit médiane i peine plus forte que les autres. - Antemnes a masstle grossissant insensiblement, peu distincte de la tige et courbée à l'extrémité. - Taille moyenne.
Chenille glabre et luisante. - Les trois nervures des supérieures très-renflées a leur origine, an moins dans les mâles. - Massue des antennes distincte de la tige. - Taille petite.

(125) Chenille pubsscente. - Norvure costale tres-renfece d̀ son origine; la médiane également renflic, quoique moins forlement. - Tuille moyenne et au-dessus.

Antennes annelées de blane seulement dans la femelle, à massue distincte de la tige, mais un peu allongée. -Taille

(12/1) $\left\{\begin{array}{l}\text { at1-dessus de la moyenue. } \\ \text { Antennes annelées dans les deux sexes, terminces brusquement en bouton aplati.-Taille moyenne. } \\ \text { Antennes annelees dans les deux sexes, it massue grossissant insensiblement et non distincte de la tige. - Ailes }\end{array}\right.$ 
(125) Antennes annelies de blize seulement dans la femelle, a massue distincte de la tige, mais un peu allongée. - Ailes inferieures fortement denlées. - Taille au-dessus de la moyenne.

GLYMENE. Fab. Uchis. God. Ulub. $165-166$. Bdv. Icon. pl. 42. 6g. 4-6. Dup. Suppl. pl. 29. fig.

Envergure, 5a mill.-Ailes d'un birun- $\mid$ Russie méridionale, Il a été fort rare dans jaunatre clair, avee la frange entrecou- Turquic, Hongrie, Vol- les collections, et n'est pée; supéricures ayant le disque fauve, hynic. En juis.

pas encore très-répandu, quoiqu'on le prenne communément sur les même plusicurs taches a même couleur, dont une ou deux marquées d'un point noir; inférieures ayec trois points noirs anté-terminaux sur des taches faures. Dessous des supérieures faure, cocadré de gris jaunâtre. Dessous des inférieures d'un jaune-verdatrc sale, avec une série anté-marginale de sept yeux noirs à iris fauve, et une éclaircic près de la côte.

․ Plus grande, plus pâle; taches apicales d'un jaune blanchâtre. Dessous des inférieures d'un cendré clair, avec la ligne médiane et l'anté-terminale distinctes.

HOXELANA. Fab. Ochs. God. Hub. 680-685. Dup. Suppl. pl. 30. fig 1-4. Bdr. Icon. pl. 45. fig. $1-3$.
Envergure, 56 mill: - Ailes d'un brun-jaunatre clair, avec la frange enquic, Crimée. En mai trecoupé; supérieures ayant le disque juin et juillet.

faure, arec une large tache costale brune, un point noir apical, et la nervure inféricure très-sinuéc; inlérieures très-dentées, avec quelques yeux bruns cerclés de grisâtre et peu marqués. Dessous des supérieures beaucoup plus clair que le dessus et sans tache costale. Dessous des inférieures cendré, avec les lignes basilaire et médiane sinuées et peu marquées en brun, l'anté-terminale double, plus claire au milieu et surmontée d'une série de sept yeux; les deux premiers et les trois derniers grands, noirs, cerclés de jaune, et les deux autres petits, gris et ì peine visibles; une tache blanchâtre au bout de la cellule.

o Plus grande, ayant la tache discoildale des supérieures coupée par les nervures et par une ligne transverse brunes, et en outi'e plusicurs taches apicales d'un jaune clair. Inférieures arec les yeux mieux marqués; dessous de celles-ci d'un gris varié de blanchatre, ayec les lignes et les yeux mieux marqués.
Grìce, Hongrie, Turfrontières de la Turquic. La femelle est plus rare que le male et vole un peu plus tard.
Ce beau Salyre n'est pas encore très-répandu dans les collections. Il a les mêmes mours que Mara. En Hongrie on le trouve volant avec Clymene, mais bien plus rarement. Il est aussi bien plus difficile de se procurer des femelles que des mâles. Les antennes de ceux-ci sont d'un brun roux sans annelures bien distinctes, avec la massue noirc ì sommité fauve, tandis que celles de la femelle sont annelées très-visiblement, comme celles de Mara, Megura, etc.

(126) Antennes annelés dans les deux sexes, termines brusquement in bouton aplatl. - Taille moyenne.

\section{(Les $V$ icicoles. Dup.)}

MOERA.

Lin.? Fab.?

Bdy. God. pl. $\Rightarrow$ sec, ig. 2 . Adrasta "Ochs. Hub. $856-859$. Le Satyre. Eugr. pl. 26. fig. 50 c. D. $\mathrm{E}$. F, et 50 bis B. C.
Envergure, 43 mill.-Ailes d'un brun| Parties méridionales| Il est moins commum jaunatre, avec une bande anté-terminale et centrales de l'Eura-que $\boldsymbol{M}$ egara. 11 arrive maculaire d'un jaune fauve; celle des pe. Mêmes époques et assez sourent que la supérieures large, coupée inférieure- localités que MA gigera: pupille inférieure de ment par une ligne brune, et marquée Chenille d'un vert l'œil apical est supprisupérieurement d'un grand oil noir bi-clair, arec une ligne mée. Lia teinte du des. pupillé, surmonté d'un autre très-petit; dorsale brune liserée de sous des inférieures celle des inférieures, qui sont légère-blanchâtre, et une ligne varie aussi du grisment dentées, étroite, composée de latérale blanchatre; tête blanc argenté au gris quatre taches, dont les deux anales ar-let pattes vertes. Vit sur|violatre un peu sau- 
|rondies et marquées chacune d'un ocil|les graminées, en avril/poudré, mais jamais aunoir. Dessous des supérieures ayant la et juin. tant que dans Mcgara, ligne qui précède l'ocil longue, briséc, Chrysalide suspen- et d'ailleurs il n'est jamais ne formant point d'angle au bout de due, d'un vert clair ou mais teinté de faure. la cellute. Dessous des inféricures d'un d'un noir verdatre, avec Il est diflicile de sagris blanchâtre uni, avec les trois lignes deux rangées de bou-voir ce qu'il faut considont lanté-terminale double, sinuée, tons fauves sur le dos. dérer comme le Mrera nullement ombrede de roux et surmontée de six yeux presque contigus, entourés de plusieurs cercles bruns et jaunâtres, et dont l'anal double.

o Plus grande, avec la bande des supérieures plus large et s'étendant sur tout le disque; celle des inférieures ayant quelquefois cinf taches. Yeux du dessous des inféricures encore plus grands et plus contigus.

\section{ADRASTA.} Dup. Suppl. pl.46. fig. $1-2$.

Mara. Ochs. $154-175$.

Le Nimusien. Engr. pl. 26 . fig. 5 i A. B.

Ochs, Treits.

Hub. $1=6$.

Bdr. Icon. pl, 44, fig. $1-5$.

Dup. Suppl. pl. 46. fig. 5-'

Maru, var. God,
Un peu plus grand; brun plus foncé ct envahissant aux supérieures la partic de la bande fauve qui est en deça de la

ligne sinueuse; cette bande d'un fative plus roux et formant aux inféricures des taches isolées et ordinairement au nombre de trois seulement. Dessous des sures d'un gris foncé, très-saupoudré de brunatre ou de violatre, et laissant mieux roir une tache plus blanche a la côte.

q Partageant tous ces caractères et ayant ì peine le disque des supéricures d'un fauye très-roussâtré. typique. Les auteurs allemands donnent le nom d'Adrasta it notre Mara ct rice rersa : aussi faudrait-il peut-être faire d'Adrasta l'espèce typique; mais le nom de MLara est si généralement adopté pour l'espèce que nous décrivons, que nous n'avons pas cru devoir le changrer.

Parties froides et Ce n'est qu'une légèmontucuses de l'Eu-revariété de Mora, arec rope.

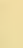

Envergure, 40 mill. - Ailes d'un Suisse, Allemagne, brun-noiratre terne, avec la frange plus Italic, Servic, Auttiche. claire; supérieures arec un oil unipu- En mai et aoot.

- pille, surmonté d'un autre plus petit et placé sur une tache d'un fauve terne au-

. dessous de laquelle se trouvent quelque-

fois deux autres taches semblables; inférieures avec une séric de taches antéterminales du même fauve, chargées de trois d̀ cinig yeux noirs. Dessous des supérieures d'un brun terne, avec les taches faures plus grandes et précédées d'une ligne formant un angle au bout de la cellule. Dessous des inférieures d'un gris un peu rosé, trís-saupoudré de noirûtre, arec les trois lignes dont la médiane éclairée à son sommet d'une tache blanchâtre, et suivie d'un rang d'yeux asser petits, ordinairement isolés, entourés de plusieurs cereles bruns et jaunatres, et dont l'anal double.

ㅇ De la même taille, arec les taches fauves plus grandes aux supérieures, et formant bande. Elle ressemble beatcoup, en dessus, au mâle de Mara.

1 MEG KIIA.

Envergure, 40 mill. - Ailes d'un Dans toute l'Europe,

Il n'est pas encore très-répandu dans les collections, parce que jusqu'ici on l'a regardé comme simple rariété de Mara; mais M. Boisduval, qui en a elcxé la chenille, pense, avec $\mathbf{M}$. Treitschke, qu'il torme une espece distincte. DC pareilles preuves et de pareils témoignages ne peuvent rien laisser a désirer sur son authenticité.

Lin. Fal, Ochs.

aune faure, arec les nervures et des li- au bord des cextrumement gnes transverses brunes; supérieures long des murs, dans les long des habitations. Hub. $377-170$. God. pl. 7 sce. fig. 3 . en ayant une plus large et plus terne sur bois, etc. En mai et On roit quelquefois un le disque, et au sommet un grand oeil juillet. 
Le Satyre. Engr. pl. |noir unipupille, surmonté d'un autre api-|chenille d'un vert d'eau, de l'œil apical des supé26. fig. $50 \mathrm{~A}$. B. cal très-petit. Inféricures légèrement avec une ligne dorsale rieures.

dentées, d'un jaune fauve, avec la pre- plus foncée et liserée de mière moitić plus foncée jusqu'd la ligne blanchatre des deux cômédiane, puis traversées d'une autre tés, une ligne latérale ligne incertaine, découpant souvent des blanchâtre, surmontée taches sur lesquelles sont quatre di cing d'une ou deux autres yeux. Bord terminal brun, traversé par lignes également blanune ligne plus claire. Dessous des infé- châtres et un peu sirieures d'un gris jaunatre saupoudré de nuées. Tête, pattes brun, avec les lignes basilaire et mé- membraneuses et poindiane bien marquées, très-dentées, éclai- tes anales vertes, pattes rées de fauve; puis six ycux tris-petits, écailleuses brunâtres. isolés, entourés de plusicurs cercles Vit en mars, avril et bruns et jaunatres. Ligne anté-termi- juin sur les graminées. nale double, très-dentéc et rousse infé- Chrysalide d'un vert ricurement.

pâle ou d'un noir ver-

+ Plus grande, dépourvue de la ligne datre, avec deux rangs plus terne des supérieures, et avec les de tubereules jaunâtres yeux des inférieures également petits et sur le dos. Suspendue isoles.

le long des murs, des arbres, etc., etc.

Tigelivs.

Bonelli. Rambur.

Plus petit, ailes un peu plus arron- Corse et Sardaigne.

dies, d'un fauve plus clair, ayec Rambur a élevé

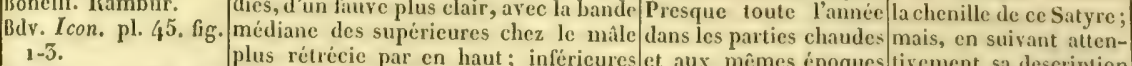

Dup. Suppl. pl. 50 . fig plus rétrécic par en haut; inféricures et aux mêmes époques mais, en suivant atten30. figr manquant absolument de la ligne brune que Megara dans les 5-7.

Paramegara.

$842-844$.

qui précéde les yeux, et ayant le bord montagnes.

IIub. terminal traversé et interrompu par une ligne fauve.

† Analogue d la précédente.

sur plusicurs chenilles de Mlegara, nous nous sommes conraincus qu'elle convenait parfaitement it beaucoup d'individus de celle-ci ; il en est de même de la chrysalide. D'unc autre part, nous arons pris aree des Megara ordidaires un individu qui offre tous les caracteres de Tigelius. Celui - ci n'est done pour nous qu'une varićté locale. Peut-être devrait-on lui restituer le nom de $\mathrm{Pa}$ rameger $\alpha$, mais nous ne sommes par sars que ce nom d'llubner soit antéricur à celui de Bonelli.

Il differe à peine de Megara par le

Dalmatic.

Il y a dans la pl. 44 dessous des inféricures, qui est d'un Pareyss. Bdv. Icon. pl. 44. fig. gris blanchatre uniforme, comme dans 4-6. M ara.

de li. Boisdural une erreur : la fig. 6 représente la var. Lyssa mâle et non le Xiphia.

(127) Antennes annelćes dans les deux sexes, à massue grossissant insensiblement el non distincle de la tige. - Ailes lrunes cn dessus. - Taille moyenne. . . . . . . . . . . . . . . . . (128)

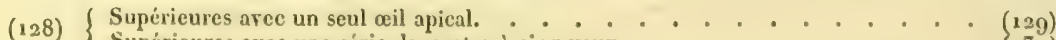
Supérieures avec une série de quatre d cinq yeux. . . . . . . . . (150)

(129) Supérieurcs avec un seul cil apical.

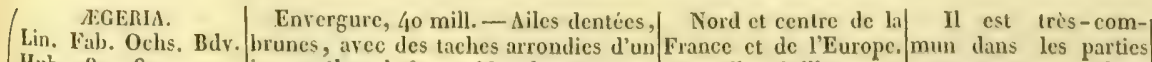

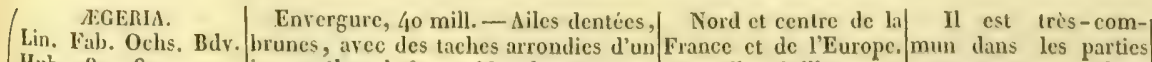
Hub. 18i-182.
jaune pále et la frange blanche. Sommet En arril et juillet.

I. 
God. pl. 8 sce. fig. 1. des supérieures marqué d'un ocil noir; Chenille ridée trans-(gées de tous les bois. Lc Tircis. Engr.

MEONe. Ochs. Treits Hub. $179-180$ Egeria. var. $\mathrm{Bd}$.

Xirais

Fab, Gorl. Bdv. Icon. pl. 44. fig. 7 .

Dup. Supplo pl. 46. fig. 5-6. des supérieures marqué d'un ocil noir;
inféricures en ayant trois ou quatre po- versalement, d'un vert sés sur les taches jaunes anté-margina-pâle, arec une ligne les. Dessous des inféricures d'un jaune dorsale d'un vert foncé, sale, arec lo bord marginal teinté de doublement liserée de gris violâtre et surmonté de quatre à blanc, et une autre ligne cinq points jaunes, cerclés de brun mais blanche au-dessus des peu nettement; ligne médiane éclairéc pattes. Tête et pattes extérieurement de jaune plus pále que vertes. Vit en mai et le fond. plus grandes arinees. Chry salide sus les taches jaunes plus grandes et plus pendae, grec los renflé et pâles.

te, arec le dos renflé et sur l'enreloppe des ailes.

Les taches, au lieu d'être d'un Midi de la France, jaune pále, sont d'un fauvc jaunfitre, Suisse, Allemagne, etc., un peu plus grandes, surtout aux infé- etc.

God. rieures, où les ycux sont plus prononcés; le dessous est, comme le dessus, plus chaud de ton et celui des inférieures est plus largement violatre; la frange est d'un brun faure.

Differe peu en dessus de la variété précédenté. Il est peut-être encore d'un

Espagne, Portugal. ton plus chaud; les supéricures sont plus aiguës au sommet et les inférieures plus prolongées d l'angle anal. En dessous, toutes les parties qui sont violatres chez Meone sunt d'un roux vif chez Xiphia. Les lignes de l'intérieur de la cellule des supéricures sont plus droites; la ligne médiane des inférieures est moins dentée, circonscrit très-bien la partic rousse de la base et est éclairée par une bande d'un blanc sale, courte, partant de la côte, où elle est plus large, et se terminant au bout de la cellule par un petit crochet.

to

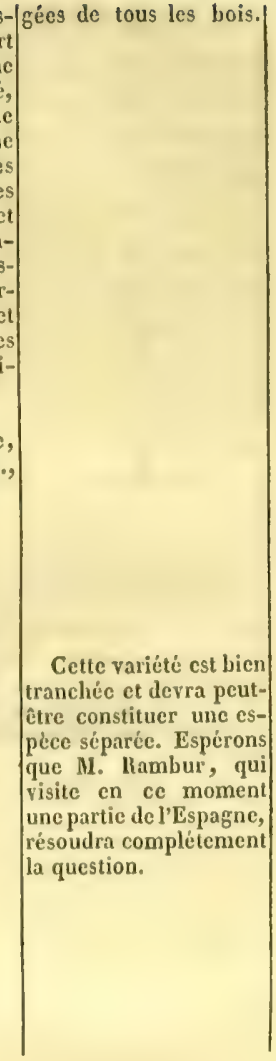

(130) Supdricures avec une sirie de deux a cing youx.

\section{(Les Ramicoles. Dup.)}

\begin{tabular}{|c|c|c|c|}
\hline $\begin{array}{l}\text { IIYPERANTHUS. } \\
\text { Lin. Fab. Ochs. Bdy. } \\
\text { God. pl. } 7 \text {. fig. } 5 . \\
\text { Polymeda. Hub. 172- } \\
\text { Le T'ristan. Engr. } \\
\text { Le Th. }\end{array}$ & \begin{tabular}{|} 
Envergure, 42 mill. - - Ailes arron- \\
dies, d'un brun-noir uni, avec quelques \\
points plus foncés et la frange d'un gris- \\
blanc; dessous d'un brun jaunâtre, avec \\
une série anté-terminale d'yeux noirs à \\
iris jaune, au nombre de deux d̀ quatre \\
aux supérieures, et aux inférieures de \\
cinq, dont les deux premiers isolés. Li- \\
gnes médiane et anté-terminale un peu \\
visibles. \\
q Plus grande, plus oculée ct dont \\
tes yeux paraissent davantage en dessus. \\
\end{tabular} & $\begin{array}{l}\text { Dans une grande par- } \\
\text { tie de l'Europe. En juin. } \\
\text { Chenille pubescente, } \\
\text { d'un gris roussattre, arec } \\
\text { une ligne dorsale brune } \\
\text { interrompue, une autre } \\
\text { latérale d'un blane jau- } \\
\text { natre et quelquefois une } \\
\text { autre cntre les deux, } \\
\text { plus claire que le fond. } \\
\text { Tête rousse, ponetuée } \\
\text { de noir; pattes roussâ- } \\
\text { tres. Vit en mai sur les } \\
\text { graminées. } \\
\text { Chrysalide non sus- } \\
\text { pendue, courte, d'un } \\
\text { jaune d'ocre, avec l'en- } \\
\text { veloppe des ailes plus } \\
\text { claire et marquée de } \\
\text { traits noirs. }\end{array}$ & $\begin{array}{l}\text { Il est fort commun } \\
\text { dans tous les bois des } \\
\text { environs de Paris et de } \\
\text { Chartres, et cependant } \\
\text { nous ne l'avons jamais } \\
\text { vu voler auprès de Châ- } \\
\text { teaudun, quoique les } \\
\text { terrains et la végétation } \\
\text { n'expliquent en aucune } \\
\text { manière cettc dispari- } \\
\text { tion subite. On rencon- } \\
\text { tre parfois des individus } \\
\text { opposés d la variété sui- } \\
\text { vinte, e'est-t-dire dont } \\
\text { les yeux sont très- } \\
\text { grands et oblongs. }\end{array}$ \\
\hline
\end{tabular}


\begin{tabular}{l|l} 
Anete. & N'en differe qu'en ce que les yeux
\end{tabular} Mull. Bork. Schn. sont remplacés par autant de petits Iyperanthus. variété points d'un blanc jaunâtre. God.

Bdv. Hub. 175 .

Le Tristan. Engr. 52 F.

DEJANIRA.

Lin. Fab. Ochs. Bdv. IIub. $170-171$. God. pl. 8. fig. 1. La Bacchante. Engr.
Envergure, 52 mill. - Ailes d'un gris-brun-jaunatre clair, arec une double ligne anté-terminale plus foncée, et Dans toule l'Europe n juin. mun. Il habite de préla frange jaunâtre. Supérieures avecune clair, avec trois lignes braǵes des bois bas et séric de cinq points noirs contigus, cer- dorsales et deux latéra- humides et les prés qui clés de jaune clair, précédés par une lesplus foncées; celles-les avoisinent. Son vol éclaircie à la côte, et dont les inféricurs ci bordées inféricure- est saccadé et sautillant, plus gros. Inférieures avec quatre à cinq ment d'unc ligne blan- et il se pose volontiers points semblables. Dessous plus clair, châtre. Tête et pattes sur les fevilles et sur les avec un trait jatne dans la cellule et une écailleuses, jaunûtres, troncs d'arbres. large bande anté-terminale, d'un jaunc membraneuses, rertes. clair aux supérieures, blanche aux in- Vitenarril sur l'ivraie férieures, et sur laquelle sont les yeux. (Lolium perenne). Ligne anté-terminale triple. ㅇ Semblable.

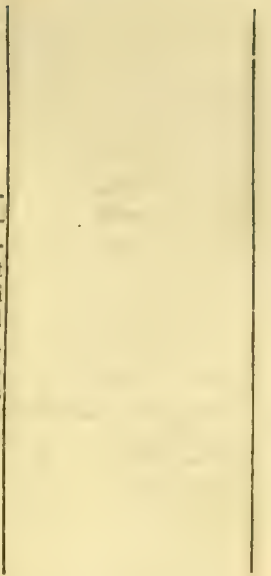

(151) Chenille.... - Toutes les nervures sans renfement brusque a leur base; la costale un peu dilatie, mais faiblement ct longuement; la médiane a peine plus forte que les autres. - Antennes d massue grossissant inscnsiblement, peu dislincte de la tige et courbce à l'extrimité. - Taille moyenne.

\section{Genre GIIONOB AS. Bdv. - Les Arcticoles. Dup.}

Ochs. AELLO.

Dup. Suppl. pl. 51. fig. $1-3$.

Bdr. Icon. pl. 56. fig 1-3. 1-3.
Hub. 5 $19-521$ et Norna féricures d'un oir écartés, et aux in$141-1 / 42$.

Ochs. God.

Hub, $763-766$.

Bdv, Icon. pl, $56, \mathrm{fig}$.

4-6.
Dup. Suppl. pl. 5 s. fig. $4-5$. terminale d'un jaune d'ocre pále, macuféricures d'un oil près de l'angle anal, accompagné interieurement d'un antreplus petit; supéricures ayant en outre un épi oblique, velu et plus foncé sur le disque. Dessous des supérieures d'un jaune d'ocre, plus obscur a la base, strié de blanc et de brun à l'cxtrémité, avec les yeux du dessus. Dessous des inférieures d'un blanc jaunâtre, très-strié de brun, arec les nervures blanches, les trois lignes a peine visibles, et un ocil à l'angle anal; massue des antennes d'un roux clair.

o l'lus grande; plus claire et plus jaunâtre en dessus, sans épi discoidal aux supéricures, quil sont plus arrondies. Yeux des mêmes ailes plus grands et plus nombreux.

Envergure, 45 mill. - Ailes $d^{\prime}$ 'un bruntrecoupée de noir, et une bande anté-

terminale d'un faure jaunâtre, marquéc aux supérieures de deux yeux écartés,

aux inférieures $d^{\prime}$ 'un seut it l'angle anal
Envergure, 45 mill. - Ailes d'un gris-
jaunátre clair, avec la frange blanche jaunâtre clair, avec la frange blanche du Tyrol et de la Sientrccoupée de noir et une bande anté- roie. En juillet. cendré jaunutre, avec la fiange grise en- Scandinavie. En juillet. ct qui manque quelquefois. Dessous des supérieures analoguc it celui d'Atllo. Dessous des inférieures d'un faure pâle très-strié de brun, avec les nervures concolores et les lignes basilaire et médiane, bien distinctes, laissant entre elles une large bande brune, "plus foncée sur ses
C'estl'espèce de cette| section la plus répandue dans les collections. Elle ne se rencontre que sur les montagnes élevées et au-dessus de la région desforêts, et aime d se poser à terre ou contre les parois des rochers. Le nombre des yeux est très-variable, surtout dans les femellcs. Le Norna d'Hubner, $1 / 41-142$, sc rapporte ici sans nul doutc. \begin{tabular}{r|r} 
Laponic, Lironic, & Il est rare dans les \\
candinavie. En juillet. & collections, mais moins
\end{tabular} cependant que les suivants. D'après II ubner et 11. Boisduval, il aurait le même ton en dessus qu'Aello, preuve qu'il rarie par le fond de la couleur, car tous les individus que nous avons rus étaient plus rembrunis que cette espèce. Dalman 'dit qu'il| 


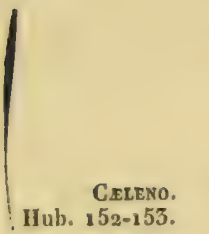

JUTTA.

Hub. 614-615. Bdv. Icon. pl. 38. fig. $1-4$.

Dup. Suppl. pl. 40. fig. 5-5. hords et éclairé de blanchâtre des deux cotes.

O Plus grande, plus pâle, d̀ ailes supérieures plus arrondies et marquées d'un petit point noir entre les deux yeus.

Le fond de la couleur est très-rembruni, les yeux sont plus petits de part et d'autre, et la bande du dessous des inférieures forme dans son milicu un angle plus saillant que dans les individus ordinaires.

Envergure, 50 mill. - Ailes $d^{3} u n$ brun roux un peu violattre, avec la frange grise, entrecoupée de noir, et une bande anté-marginale très-maculaire, d'un . fauve jaunâtre. Supérieures très-aiguës au sommet, ayant un large épi brun et velu sur le disque, et la bande chargée de trois points noirs rarement pupillés. Inférieures avec cette bande egalement maculaire et marquée de deux points noirs près de l'angle anal. Dessous des supérieures d'un gris jaunâtre, avec la bande plus claire et l'angle apical strié de gris. Dessous des inférieures d'un gris cendré légèrement violätre, finement strié de brun, arec les lignes basilaire et médiane sensibles, mais pru marquées et éclairées de gris violatre plus clair que le fond. Massue des antennes d'un roux foncé en dessous.

q Plus grande, plus arrondie, ayant les points plus gros, plus souvent ocellés et parfois au nombre de quatre aux supérieures, qui n'ont point d'épi discoidal. Dessous des inférieures un peu moins violatre et à lignes oblitéries et indiquées seulement par trois ou quatre taches plus claircs que le fond.

\section{BaLder.}

Bdr. Icon. pl. 39. fig. 1-3.

Dup. Suppl. pl. 49 .

Beaucoup plus petit (40 mill.).-Supéricures dépourvues de l'épi discoîdal noirâtre. Dessous des inférieures ayant fig. 4-5.

\section{. la bir} sible du côté interne. Ligne anté-terminale plus prononcée. la bande un peu moins sinueuse, forte-
ment dentéc du côté cxterne et peu sen-

Idem.

varie aussi pour le nombre des points ocellés.

C'est à peine une variété, et on retrouve tous ses passages arec Norna.

Laponic, environs de Il est encore extrêTorneo et de Lyckscle. mement rare dans les En juillet.

collections. Nous en avons vu quatre individus.

\section{Cap-Nord.}

M. Duponchel pense qu'il n'est qu'une variété plus petite de Jutta, et M. Boisduval le regarde comme espèce séparée. Pour nous, qui ne l'avons pas ru en nature, nous ne pouvons nous prononcer it cet ígard. Il est trèsrare, et se trouve anssi au Groënland et en Islande.

TARPEIA.

Esp. Ochs.

Envergure, 45 mill. - Ailes d'un

Russie méridionalc fauve sale, arec les nerrures plus fon-entre le Volga et les cées et la frange entrecoupie de noiritre. monts Ourals.

Supéricures aiguës au sommet, arecla Tarpeius. Fab. God. Dup. Suppl. pl.51. fig. - base un peu obscure et une bordure d'un brun roussâtre, précédéc d'une série de cinf points noirs, aveugles. Inférieures ayant la base légèrement brunâtre jusqu'd la ligne médiane, et une bordure comme les supérieures, précédée de quatre points noirs. Dessous des
Il n'existe pas en France. Notre description est faite sur une figure fort exacte que ii. Lefebvre a fait faire a Vienne, lors de son royage en Autriche. Celte figure représente une femelle, e'est pourquoi nous nvons mieux áimé la décrirc seule 
|supéricures fauve, strié de brun, arec| le sommet jaunatre, et une ligne brunâtre formant un angle aigu au bout de la cellule. Dessous des inféricures avec les nervures blanchatres, la ligne médiane bien marquée, éclairée de jaune clair, et la ligne basilaire un peu indiquée par une éclaircie de la même couleur, qui se voit en transparence en dessus. Points pareillement aveugles; antennes d'un roux clair. $q$.

BOOTES. Envergure, 45 mill. - Ailes minces, $\mathrm{Bdv}$. Icon. pl, 37. fig. d'un gris-brun jaunâtre; les supéricures 4-6.

tris-aiguës au sommet; les inférieures Dup. Suppl.pl. 32. fig. légèrement dentées, aree le disque et 3-5.

Treits. Suppl. la bordure plus foncés; cctte dernière
surmontée d'un rang de taches incertaines, mais bien sensibles, d'un jaune d'ocre. Dessous des supéricures plus jaunatre que le dessus, avec le sommet blanchatre, strić de brun ct marqué presque toujours d'un point blanc. Cellule fermée par un trait noiratre, renfermant quelquefois une ligne et suivie d'une autre ligne semblable, formant un angle aigu tris-prolongé sur la quatrième nerrure. Dessous des inférieures gris, strie de brun, avec les nervures blanchitres et les lignes basilaire et médiane nettement coupées, laissant entre elles une bande d'un brun noir, eclairée de blanc des deux cólés; ligne anté-terminale formée d'une suite de traits interrompus.

f Plus grande, plus arrondie, plus jaunatre, avec les bandes du dessous visibles en dessus, même aux supéricures.

BORE.

Envergure, 45 mill. - Ailes très-56? $154-156$. minces, d'un gris jaunattre pâle, avec Bdv. Icon, pl. 5y. fig. plus claire, surtout aux inféricures. $1-3$. Dessous des supéricures très-strié de Dup. Suppl. pl. 32. brun et blanchatre au sommet. Dessous fig. 1-2. Fortunatus. Fab. God. avec les nervures blanchâtres et la ligne médiane plus foncée, denticulée et éclairée de blanchatre; ligne basilaire quelquefois également marquée et éclairée, mais moins distinctement; pattes et antennes d'un jaune roussître.

P Plus grande, plus arrondie, un peu strice en dessus.

OENo *

Bdv. pl. $59 \cdot$ fig. 4-6. Dup. Suppl. pl. 49 . fig. $1-5$.
La coulcur du fond est plus foncéc et les supéricures portent qquelqquefois unc série de taches jaunattes antémarginales a peine sensibles. La frange est plus distinctement entrecoupée. I.e dessous que de donner la des-1 cription du mâle d'aprés les auteurs, ce qui ne serait qu'une répétition inutile. La figure qu'en a donnée M. Duponchel a été également copiéc sar ce dessin, mais l"enluminure a rendu le fauve trop vif et le brun. trop tranché.

Cap-Nord.

11 est tres-rare. On le trouve également an Groënland et an Lalsrador. On remaique quelquefois sous les inféricures, au-dessus de la ligne anté-terminale, une série de petits points blancs. Il doit présenter du reste plusieurs variétés, mais les espèces hyperboréennes sont si rares dans les collections, qu'on ne peut les étudier que sur un petit nombre d'individus. Nous n'en arons vu qu'une paire de cette espèce.

Alpes de la Laponie. N'ayant eu 'à notre En juillet.

disposition qu'un scul individu de cette espèce, nous n'arons pu bien juger des diflérences qu'il présente avec les especes si roisines qu'on a décrites dans ces dernicrs temps. Nous pensons que cet individu est le Fortunatus de Godart et Fab., ou Bore de Dalman, ctc., elc.; mais nous n'oserions assurer qu'il soit celui de M. Boisdural, lequel nous semble différer du Bore des auteurs.

Laponie.

Nous n'avons vu que deux femelles parfaitement conservées de ce Satyre, et il nous est impossible de nous pro-

d'M. Boisduval décrit sous le nom d'Also le male d'un Satyre trís-voisin de ceux-ci; mais, comme il n'a encore eté pris que dans la Siberie et aux Litats-Unis d'Amérique, nous ne pensons pas qu'il doive être considéré comme européen. 
des inférieures est plus strié; la bande transyerse y est moins sensible que dans Bore. Les antennes sont brunes en dessus, d'un ferrugincux foncé en dessous (cependant $\mathbf{M}$. Lefebrre a ru un individu qui les arait tout-à-fait semblables id celles de Bore). Le corps est garni de poils trés-noirs.
Inoncer sur sa validitê sans en aroir comparé un certain nombre des deux sexes arec Bore. Nous devons dire senlement que ces deux femelles présentent la plus grande analogie avec ce dernicr.

(153) Chenille glabre et luisante. - Les trois nervurcs des supérieurcs trìs-renflées d leur origine, au moins dans les mâles. -Massuc des antennes distincte de la tige.-Taille petite.

(Les deux premieres nerrures seulement renflées i leur base dans la femelle.-Massue des antennes globuleuse et en

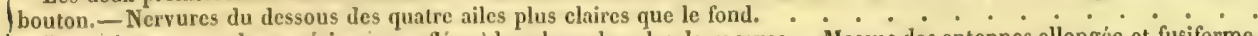
Les trois nerrures des supérieures renflées il leur base dans les deux sexes.-Massue des antennes allongée et fusiforme. -Dessous des ailes ayant les nerrures concolores.

(154) Les deux premieres neroures seulement renflées d leur base dans la femelle. - Massue des antennes globuleuse ct en bouton. -Nervures du dessous des quatre ailes plus claires que le fond.

\begin{tabular}{|c|c|c|c|}
\hline $\begin{array}{l}\text { PHRYNE. } \\
\text { Ochs. } \\
\text { Hub. 200-201.708-gog. } \\
\text { Bdv. Icon. pl. 45. 6g. } \\
\text { 4-6. } \\
\text { Phryneus. Fab. God. } \\
\text { Dup. Suppl. pl. 33. fig. } \\
\text { s-4. } \\
\text { Le Phryné. Engr. }\end{array}$ & 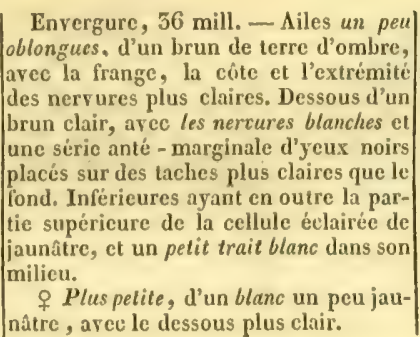 & $\begin{array}{l}\text { Russic méridionale, } \\
\text { bords du Volga, Cri- } \\
\text { mée. En juin. }\end{array}$ & $\begin{array}{l}\text { Il est très-rare dans } \\
\text { les collections; nous } \\
\text { n'en arons vu qu'une } \\
\text { seule paire, mais il sem- } \\
\text { ble varier fort peu. Il } \\
\text { se rapproche d'A fra par } \\
\text { le dessous des inférieu- } \\
\text { res et surtout par le } \\
\text { trait de la cellule, mais } \\
\text { il s'en éloigne sous tous } \\
\text { les autres rapports. }\end{array}$ \\
\hline
\end{tabular}

(135) Les trois nervures des supéricures renflées à leur base dans les deux sexes. - Massue des antennes allongée ct fusiforme. - Dessous des ailes ayant les nervures concolores.

\section{(Les Dumicoles. Dup. - Petits Satyres vulgairement.)}

OEDIPUS.

Fab. Ochs. Treits.

God. pl. 19 s. fir. 5-6. OEdipus. Bdv. Dup.

Pylarge. Hub. 2द5-2,6. 702-705.

Miris. Fab.
IIERO.

I.in. Ochs. Bdr.

Hub. $252-253$.

Var. accid. 849-85o.

God. pl. 8 sec. fig. 2.

Sabaus. Fab.

L. Matibic. Ingr.
Envergure, 37 mill.-Ailes entières, arrondies, d'un brun noiratre uni. Des-Hongric, centre de la sous d'un brun jaunatre clair, avec une France. Dans les bois. ligne anté-terminale coulcur de plomb, I la fin de juin.

brillante. Supéricures ayant cette ligne précédée, près du bord interne, d'un ou deux yeux noirs à iris jaune. Inférieures en ayant une série de six plus grands, dont celui de la côte isole; ces yeux souvent précédés d'une bande courte et ondulée d'un jaune clair ou d'un gris argenté.

ㅇ Ayant deux des yeux apparents aux inferieures en dessus, et un troisième aux supérieures en dessous.

Envergure, 5 f mill. - Ailes d'un Nord et centre de la brun noirâtre; les supérieures entieres, France et de l'Europe. arec un tres-petit point noir apical cer-En mai et juin.

clé de fauve; les inférieures très-légèrement polygonées, avec trois it quatre points semblables, dont deux beaucoup plus gros, et un trait fauve a l'angle anal. Dessous d'un brun plus clair, arec une
Il est commun aux environs de Beaugency (Loiret), mais ses localités sont assez restreintes en France. Nous pensons avec Godart que le $S$. Miris de Fabricius n'est autre que la femelle de cette espéce.

Il n'est pas rare dans certaines localités des environs de Paris, mais a mesure qu'on approche du midi il devient moins abondant. Nous ne l'avons trourè qu'une seule fois aux 
|ligne anté-terminale couleur de plomb. Inférieures marquées au-dessus de six yeux noirs a iris d'un faure rouge, précédés d'une bande blanchatre. Bord terminal longé par une ligne également d'un rouge fauve.

o $\Delta$ yant les yeux micux marqués, et ordinairement aux supíricures un point fauve assez distant de l'oil apical, qui est précédé en dessous d'une ligne blanchatre.

LEANDER.

Fab. Ochs. God. Dup. Suppl. pl. 23. fig. 5-7. Bdv. Icon. pl. 45. fig. 7-8.

Clite. Hub. 526-527. $747-748$.

ARCANIUS,

Jin. Fab. Bdv. God. pl. 8. fig. 3. Arcania. Ochs. Hub. 240-2/2. Le Cephale. Engr.

Herbst.

AMARILLIS.

Eyersmann.
Envergure, 38 mill.-Ailes entières; les supérieures un peu aiguës au sommet, faures, avec une large bordure, le bord interne et l'extrémité des nerrures d'un brun noirâtre, et marquées d'un point noir apical. Inférieures d'un - brun noiratre, arec une tacke faure d l'angle anal, et une série de points noirs anté-lerminaux. Dessous d'un faure mêlé de gris, avec une ligne anté-terminale plombée; inférieures ayant au - dessus une bande d'un fauve plus vif, surmontée d'une séric d'ycux noirs, petits, égaux, bien alignés et légèrement cerclés de jaune clair.

o Plus grarde, plus arrondic, ayant les ailes supérieures entièrement fauves, arec une bordure noire étroite, et aux inférieures une bande anté-terminale fauve, diviséc par les nerrures et sur laquelle ressortent bien les points noirs. Ycux du dessous des inférieures plus largement cerclés de jaune.

Envergure, 36 mill. - Ailes d'un brun noirâtre. Supéricures ayant le dis- tie de l'Europe. En juin que fauve, inféricures ayant un trait et juillet. fauve d l'angle anal. Dessous des supérieures fauve, avec une ligne anté-ter- une ligne dorsale noi- ric comme tous ses conminale plombée et un ocil apical noir a ratre, liscrée de jau-génères pour le nombre iris jaune et précédé d'un trait de cette ne des deux côtés, puis et surtout pour la taille couleur. Dessous des inféricures d'un une autre ligne sembla- des taches oculées.

gris jaunâtre jusqu'a la ligne médiane, ble, liserée ausside jauqui est nettcment coupde et largemeni nâtre inférieurement, éclairie de blanc jaunatre, puis ayant le puis une ligne latérale bord anté-terminal $d^{\prime}$ un faure foncé, tra- jaune au-dessus des patversé par une ligne plombée et surmon-tes, parfois surmontée té de trois à six yeux noirs, dont trois d'une ligne pareille $\mathrm{a}$ la plus grands, surtout celui de la côte, seconde, mais plus fine qui est très-rentrant en dedans et pres- et peu visible. Stigmaque perdu dans la partie grise. les roussâtres; tête et

f Semblable, mais ayant souvent un pattes vertes. Vit en petit point noir cerclé de faure près de mai sur les graminées. l'angle apical des supéricures.

Envergure, 38 mill. - arec une ligne fine anté-marginale juillet. interrompue et précédée d'une série de points noirs inégaux et peu marqués. Dessous des supérieures faure, avec une ligue anté-marginale plombéc, surmontéc de quatre ou cinq yeux noirs it due, courte, d'un vert jaunâtre, quelquefois marquée d'une ou deux lignes noires.

Jenvirons de Châteaudun, bien que nous l'y ayons souvent cherché.

Le point apical est souyent accompagné d'un second, surtout en dessous. Ce Satyre est rare, et la plupart des collections ne le possćdaient pas; mais depuis quelquesannées les mar. chands allemands l'ont un peu plus répandu.

Il est commun dans nos bois. In dessus le

hones noires.

juillet.
Ourals. En $\begin{gathered}\text { Il n'existc pas en } \\ \text { France, et nous l'arons }\end{gathered}$ décrit sur la figure et la description que M. Eversmann en donne dans les Mémoires de la Société des naturalismile se rapproche de 
firis jaune, précédés cux-mêmes d'un trait jaune. Dessous des inférieures d'un gris juunâtre, arec une ligne plombéc surmontée d'une bande fauve, sur laquelle sont cinq yeux noirs cerclés de jaune, précédés eux-mêmes d'une ligne d'un blanc argenté, au bout de laquelle est un sixième oeil, près de la côte, perdu dans la partie grise et mal aligné avec les autres.

CORINNA.

Ochs. Bdv.

Hub. 536-55 7 .

Corinnus. God. pl. $22 \mathrm{~T}$. fig. $7-8$.

Ochs. Bdr.

1)ORUS.

God. pl. 20 т. fig. 5-6.

Dorion. Ilub. 247-248. Le Palémon. Engr.

PIILEA.

Hub. $254-255$.

Bdv. God. pl. 20 T. fig. $1-2$.

Salyrion. Ochs.

Envergurc, 29 mill. - Ailes d'un fauve vif; supéricures avee une bordure et un grand ail apical d'un brun noir; infégrand et diris d'un jaune clair. die, plus pâle, arec les supérieures moins marquées de noir.

Envergure, 32 mill. - Ailes supé-

clé de fauve. Inférieures fauves, avec rie de points noirs arqués et dont la convexité lournée vers la base. Bessous d'un jaune d'ocre grisâtre, avec unè ligne placé sur un espace plus clair, coupé intérieurement par une ligne plus foncée. Dessous des inférieures d'un cendré jaunâtre jusqu'd la ligne médiane, puis d'un jaune clair, avec la ligne plombée festonnée et surmontée de six yeux disposís très-irrégulièrement.

온 lyant les supérieures fauves en dessus, avec une bordure brune.

Envergure, 52 mill. - Ailes entières, ricures largement lavées de celte couleur a la cóte et marquées d'une série antélés. Dessous faure, arec une ligne antéterminale plombée; supéricures ayant d'une autre plus foncée, le plus petit du genre, l'cil apical cerclé de jaune clair; infé-puis au-dessus des pat-varie beaucoup. Comricures avee la ligne médiane sinuée, tes une ligne jaunâtre raeses analogues, il prééclairée de jaune clair, et une série de sinuée. Tête d'un vert féré les endroits remplis cing à six yeux, dont le costal plus obscur; pattes écailleu-d'herbes sèches, et sa

o Un peu plus grande, plus arronrieures un brun clair, avec un gros point apical cer-juillet. la côte largement lavée de brun et une séplombée; supérieures avec l'œil apical arrondies, $d$ 'un brun clair. Supérieures du Tyrol, etc., etc. Én ayant le disque largement teinté de juillet.

fauye, sans ceil apical; inféricures ayant un trait fauve à l'angle anal. Dessous des supérieures d'un fauve terne, avec le sommet et le bord marginal d'un grisverditre clair. Dessous des inféricures de cette dernière couleur jusqu'd la ligne médiane, qui est bien détachee et éclairéc

Chrysalide suspen- Le papillon est com- tes de Moscow. Il n'y est point question du sexe, et nous ignorons si c'est un mîte ou une femelle ; mais il est évident que ce Satyre ne se rapporte point à Leander, comme on l'a cru jusqu'ici. La ligne argentée qui surmonte les yeux du dessous des inféricures manque quelquefois.

Corse, Sardaigne, Si- Souvent les nerrures le. En juin et aont. du dessous des inférieuChenille verte, avec res sont plus claires que ne ligne plus foncée et le fond; quelquefois serée de vert pále sur même on remarque da la ses roussatres, mem- chenille même, d'après les observations de M. Vit en arril, mai, juil- Rambur, dédaigne les let et aout sur le Carex graminées qui croissent gynomane et le Triticum dans les lieux frais et cespitosum. humides. due, courte, d'un gris mun en Corse et en roussattre, variée de Sardaigne, mais il est noir et de blanchâtre. encore assez peu répandu dans les collections.

Espagne, Portugal,

Il cst très-commun et varic assez, principalement pour la grandeur des yeux, qui sont quelquefois réduits à de très-petits points.

Alpes de la Suisse,

Il est moins commun que le précédent et le suivant. Il varic un peu comme ce dernier pour la teinte du dessus. 
0chs. Bdv.

IPIIIS.

IIub. $249-25$ I. arrondies, d'un brun clair. Supérieures

Gou. 0 T. fig. 3. ve, sans ail upical. Inférieures ayant or-

50 C. D.

DAVUS.

Lin. Fab. Ochs. Bdv.

God. pl. 21-v. fig. $1-2$.

Tullia. Hub, 243-244.

Le Daphnis. Engr.

PAMPIILUS.

Lin. Fab. Ochs. Bdy.

God. pl. 8 scc. fir. 3.

Nephele. Hub. 237 239.

Le Procris. Ingr.

Linvergure, 32 mill.-Ailes entières, ries d'une série de quatre d̀ cinq petits yeux cercles de gris blanc et dont le costal plus gros et rejete en dedans.

$q$ Différant du ơ par les mêmes caracteres que celle de Plailea.

Envergure 55 mill. Ailes d'un jaune fauve. Supérieures plus claires sur le

disque, arec un très-petit point bruná-

tre cerclé de faure. Inférieures plus sombres, avec un ou deux points parcils près de l'angle anal. Dessous des supérieures fauve, avec le sommet gris et deux ou trois petits yeux précédés d'une ligne plus claire. Dessous des inférieures d'un gris jaunatre, avec la ligne médiane indique seulement par deux ou trois taches blanchatres, suivies d'unc série d'yeux noirs cerclés de jaune. o Scmblable. d'unc bande d'un blanc jaunâtre, continue, large et marquée de six yeux bien alignés. Bord terminal entièrement $d^{\prime} u n$ fauve roussatre, traversé par une ligne plombéce.

\& Ayant les supérieures fauves, légèrement ombrées de gris-brun clair près du bord terminal, et les inféricures d'un gris-brun, avec une petite ligne antéterminale fauve.

Suisse, Allemagne, Suède, Est de la France, ctc. En juín.

Chenille verte, poinOn voit sonrent sous es ailes inférieures, surtout chez les individus pris en Suisse, une dinairement un trait fauve à l'angle anal. tillée de jaunâtre sur le petite ligne fauve i Dessous des supérieures d'un fauve dos, avee une lione dor-l'angle anal dans les terne, avec le sommet et le bord mar- sale noirútre on d'un máles, et les femelles ginal d'un gris-verdâtre clair. Dessous vert foncé. Tête et pat- présentent parfois queldes inférieures cntiirement de cette der- tes rertes. Stigmates ques points fauves auniere couleur, ayant la ligne médiane roux. Vit sur les grami- dessus de cette ligne en non visible et seulement indiquéc par nées en avril et mai. dessus. L'Iphis est comdeux taches irrégulières blanchatres, sui- Clirysalide suspendue, mun; pour l'avoir frais verte, arec un double il faut le chasser en juin, rang de tubercules blan- et non en juillet comme châtres sur le dos.

l'indiquent quelques auteurs.

Nord ct Est de l'Europe. In juin.

Envergure 29 mill.-Ailes d'un janne Dans toute l'Europe. fauve, arec une bande terminale brunâ- Endroils secs et herbus. tre, ordinairement peu prononcée. Su- En mai et juillet.

Il est très-commun et périeures ayant en outre a l'angle apical Chenille d'un raric assez, surtout pour un point brunatre, ordinairement petit pomme, arec une ligne pour le plus ou le moins et quelquelois tout-it-fait effacé. Dessous dorsale d'un vert foncé de netteté des points du d'un gris verdâtre, arec le disque des liserée deblanchâtre des dessous dos inféricures. supéricures fauve et marqué à l'angle deux côtés, et une ligne On le troure pendant apical $d^{\prime} u n$ point ocellé; les inférieures latérale semblable, mais presque toute la belle un peu plus foncées jusqu'à la ligne mé- plus étroite et liserée saison.

diane, qui est visible dans toute sa lon-sculement inférieuregueur, et qui forme au bout de la cellu-ment. Tête et pattes d'un le une saillic éclairće de blane jaunâtre. Fert jaunâtre; pointes Ligne anté-terminale it peine sensible, anales roussattres. Vit en brune et surmontée de petites taches $l \delta$ - arril, mai, aoot et sepgèrement ocellées et souvent presque insen- tembre, sur les gramisibles, de la mème couleur.

o Semblable.
Très-sourent les taches blanchâtres du dessous des inférieures sont marquées en clair en dessus, surtout chez les praics. Il aime les montagnes.
Chrysalide suspendue, d'un vert-pale uni ou rarié de quelques lignes noires sur l'enreloppe des ailes. 
Lycuss. | Bande terminale des quatre ailes bru- France méridionale, Certains exemplaires Ochs. God. pl. 20 x. no et bien arrêtie; point upical gros et Espagne, Portugal, Si- de ce Satyre paraissent fig. 9-10. $\quad$ et bien marqué; une série de petits cile, etc. En nati, juillet d'alsord très-llistincts de Pamphila. Hub. 557-points bruns anté-marginaux aux infé- et aoot.

558. rieures. Dessous d'un gris blanchâtre carné, avec la ligne médiane des inférieures bien marquée, sinuéuse, mais sans saillic principale, et ćclairće dans toute sa longueur; points ocellés plus nombreux. ㅇ Semblable.

Pamphilus; mais ec dernier varie tellement suirant les localités, qu'on ne saurait fairo une espece de Lyllus avant la décourerte de sa clienille. Il est très-commun. La femelle offre quelquefois une ligne plombée sous les ailes supérieures, et ses ailes inférieures sont légèreiment dentées.

(156) Jambes postérieures ayant deux paires d'épines. - Ailes non parallèles verticalement dans le repos. . . . .

\title{
Tribu III. IIESPERIDI (IIEspérides).
}

\author{
(Latr. Heteropterus. Duméril. - Involuti (tribu des). Bdv.)
}

Cirractères principaux. - Chenilles tortriciformes", minces et delicates, vivant à l'abri du contact de l'air, soit dans des fouilles replices, soit dans l'interieur des tiges. - Chrysalides enveloppées dans des feuilles roulées. - Six pattes ambulatoires, les posterieures munies de deux paires d'épines ou ergots. - Cellule des ailes inférieures ouverte.

Ciractires secondaires. - Corsclet robuste. - Tite aussi grosse que lui.-Abiomen des mâles pourcu de poils a son"cxirimite. - Ailes presque toujours musculeuses et operant un vol vif et rapide, quelquefois à l'ardeur du soleil, plus souvent vers l'apris-midi.

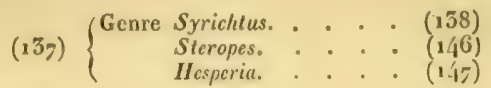

(138) Genre XVIII. SYRICHTUS (sYricite).

$$
\text { (Nobis. Syrichtus et Thanaos. Bdv. - Syrichtus, Thanaos et Spilothyrus. Dup.) }
$$

Caractires principaux. - Chenilles dête grosse et saillante et a premier anneau tris-étranglé. - Chrysalide conique, sans aucune pointe que celle de l'extrimile posterieure: - Les quatre ailes d peu prés horizontales dans le repos. - Palpes écartés, velus, leur dernier article nu et tris visible. - Corps robuste. - Ailes superieures ayant le plus souvent un repli ** d la côte dans les mâles.

Caractìres secondaires. - Y ol vif et rapide. - Ailes d fond brun, avec de petites laches blanches ou vitrdes. - Taille petite. . . . .

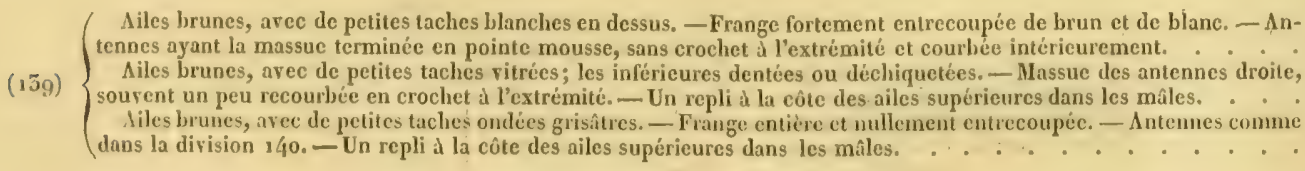

- Ce nubl, cmplnye par Dahman, cas acterise fort bien ces chenilles, dont la peau fine et transparente comme celle de la plupart des Toriricides, soulfriail de l'influence de l'air.

*- Ce repli, qui semble n’avoir pas encore été bien observé, est analogue à celui que présentent les ailes inféricures de quelques Papilio exotiques. Quand on Je relère, l'intérieur en parait canaliculé et jaunâtre, tandis que la partie relevée saillit notablement sur la cote. Il n'est pas plus facile d'en deviner l'usage que de ceux des Papilio que nous venons de citer, ou de la poche qu'on voit aux ailes inférieures dans certaines cepèces du genre Danais, Ces differents organes semblont être le gastage cxclusif des mâles. 

(140) Ailes brunes, avce de petites taches blanches cn dessus. - Frange fortement cntrccoupcic de brun cl de blanc. - Antennes ayani la massue
terminée cn pointe mousse, sans crochet a l'extrémité et courbée intéricurement.

Genre SYMICHTUS. Bdv. Dup.

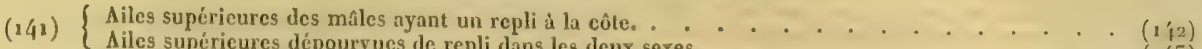

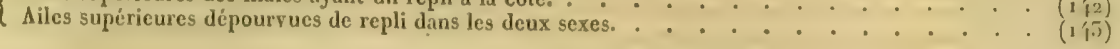

(142) Ailes supéricures des mâles ayant un repli d la côte.

SID $\pi$. Fab. Ochs. Bdv. II ub. 468 . IIesp. cle Sida. God. $\mathrm{pl}$ 27 A a. fig. 5-6. Le Chamarre. Engr.

\section{CAITHAMI.}

Ochs. Hub. $720-725$. Envergure 50 mill. - Ailes d'un gris Tesselum.God. Encycl. brunâtre très-saupoudré de blanchâlre. Hesp. Plain-Chant. Supéricures avec beaucoup de taches God.pl. 12, fig. 4-5. blanches assez grandes, dont une dans Le Bigarré. Engr.

\section{la cellule, et neuf autres composant unc} séric transperse et très-sinuée, bien nettement coupees, les autres incertaines et plus ou moins marquées, celles de la séric anté-terminale toujours distinctes. Inférieures tres-développes, entières ou à peine sinuées près de l'angle anal, arec deux séries de taches blanches plus ou moins marquées et quelquefois un point blane it la base. Dessous des supéricures ayant à l'angle apical, qui est blanchatre, deux petites taches grises en anneau allonge et longitudinales. Dessous des inféricures d'un gris clair, tirant parfois sur le verdâtre ou le roussatre, avec le bord marginal blanchatre et trois séries de taches blanches cerclées de gris fonce, dont celles de la série postérieure fondues dans le bord terminal, et étant, savoir, la deuxieme à partir de la côte bifide intérieurement, et celles qui aroisinent l'angle anal, lunulées et marquées à la base chacune d'un point de la coulcur du fond. Antennes ayant le côté interne de la massuc d'un ferrugineux foncd ou brun.

q'antôtsemblable, tantôt plus foncè, etalors saupoudrée de jaune verdatre au lieu de blane, avec la série anté-terminale de taches anx supérieures, et toutes celles des inférieures peu marquées et jaunatres; dessous des inférieures à dessins plus marqqués et plus verdâtres.

Tesserlem. Ochs. Treits. Hub. $469-470$.
Plus grand (55 mill.). Ailes d'une teinte plus foncée, arec les poils et les atomes moins nombreux et plus jaunatres, ct conséquemment les taches plus|
Italie, Hongrie, Tur-1 Une description trèsfuie, Russie méridiona- Ionmue de relle erpiece serait inutile; les bandes jaunes du dessous la feront loujours sarement distinguer de ses congréneres. Quelquefois la bande interne se ramifie, de maniere ì en former une troisieme trèscourte di la base de l'aile. Cette espéce est rare et peu répandue dans les collections.

Dans presrue toute Il est commun aux Europe. En mai et environs de Paris. Nous ont.

l'avons décrit longuement parce que beaucoup d'amateurs le possedent sous le nom de Tessetum, et qu'une deseription bien précise peut seulo le faire nettement distinguer. Il varic beaucoup, quoique moins que Fritillum, et certaines de ses rarićtés ne sont pas moins tifficiles ì étudier que celles de ce dernier. Le genre Syrichtus, au reste, sera long-temps le désespoir des entomologistes qui ne se fient pas. au premier coup d'oil pour nommer une espèce.

Nota. Hubnex figure sous le nom de Tartarus, 716-717, un Syrichtus exolique et dont la patric est le jirésil.

France méridionale. Jusqu'à ce qu'on ait tronvé la chenille de ce Syrichtus, nous ne saurions le regarder comme| 
filitiLu,

Ochs. Dalm.

God, pl. 28 b b. fig. 1-3.

Alveus. IIub. 461-463.

Fritillum. Fah.?

Malrce. Lin.?

ALYevs.

Ochs. Hub. 506. Bdr. I con, pl. 46 . Gg. $1-3$. netles et frappant davantage au premier coup-d'oil. Supéricurcs ayant la tache du bout de la cellule plus longue, dentée intérieurement et surmontée des trois traits blancs bien marqués. Taches de la série transverse presque toutes lunulées en dehors; les deux du hord interne presque réunies. $\Delta$ iles inférieures peutêtre un peu moins larges et semblant moins sinuées à l'angle anal; dessous arec le dessin plus prononcé. Bandes des inféricures rerdâtres, très-nettement bordées, avec les taches blanches plus grandes. Antennes, corps, palpes, etc., comme dans Carthami.

Envergure 27 mill. au moins. - Ailes d'un brun assez foncé. Supéricuresayant la base largement saupoudrée de jaune Bois secs et montueur. verdatre et parsemées de taches blan- En mai et aoat. ches, petites et isolées, celles de la série anté-terminale non visibles et remplacées par de larges espaces saupoudrés de jaune cerdalte. Inféricures avec un point it la base et deux séries de taches d'un blanc sali de jaune verdatre; celle du bout de la cellule plus large et bifide extéricurement. Dessous des supérieures ayant au bout de la cellule une tache en anneau bien prononcée. Dessous des inférieures ' $d$ 'un jaune-verdâtre obscur, avec trois séries de taches blanches, la basilaire composée de trois taches dont la supéricure plus grande; la médiane for. unant une bande à peine interrompue et plus large jusqu'd moitié à partir de la côte. Antennes ì massue, d'un roux clair intéricurement.

q Semblable, mais ayant sourent les taches du dessus des inférieures un peu plus marquées.

Ne differe sensiblement de Fritillum qu'en ce que les taches des supéricures se, du Tyrol et de la sont tris-petites et que les inférieures de Norwìge.

la série transterse n'existent pas, non plus que celle du bord interne. Le dessous des inférieures offre le même dessin que Fritillum, mais les taches sont plus incertaines et plusieurs d'entie elles sont oblitérées. Le dessus de ces mêmes ailes n'offre aucune tache, parfois cependant quelques restiges, surtout dans la . distinct de Carthami, dont il differe très-peu, si ce n'est par la taile; encore avons nous vu des variétés de Carthamiqui atteignaient presque celle de Tesselum. L'Hespérie figuréc par 31. Duponchel dans son supplément sous le nom le Curthami et décrite sous cclui d'Alveus, ne nous semble pas se rap. porter, comme il le présume, it cette rariélé, qui est fort rare, et dont nous n'avons pu voir qu'un seul individu.

Il varie prodigicusement, même pour la taille. La figure de Godart est trés-bonne et représente parfaitement les individus de nos environs; mais nous l'avons recu de IIongrie sous lo nom d'Alicets, et complétement semblable à la figure d'llubner que nous citons; cnfin, nous en avons pris un aux environs de Châteaudun qui se rapproche beaucoup d'Alreus Oclis., et qui dépasse à peine la taille de l' $A$ treolus.Quoique nous ayons trouvé le Fritillum plusicurs fois dans nos environs, on ne saurait dire qu'il y est commun, comme Godart le prétend; mais M. Boisduyal nons semble faire une erreur en supposant que Godart l'a confondu avec Carthami. Les descriptions et figures de Godart sont au contraire très-précises, comme nous l'arons dit plus haut.

Nous arons ru plusieurs individus de cette prétendue espèce, et. nous demeurons convaincus avec M. Treitschke qu'clle n'est qu'une variétć de Fritillum, modifiéc par la diflérence des localités. Nous avons $\mathrm{ru}$, comme l'entomologiste que nous renons de citer, des individus formant passage d'Alceus à Fritillum. 


\section{G. SYRICHTUS.}

ALVEOLUS.

Ochs. Bdv.

Ilub. $466-467$.

Var. accid. 847-848.

IIesp. du Chardon. God. pl. 12 sec. fig. 3.

Malva. Dalm. Lin.?

Le Plant-Chant. Engr 97 E. $F$.

Fab.

ILAYATERe.

Althece. Bork.

Alveolus. II ub. 597.

Le Plain-Chant, var.

Engr. 97 G. II.

Oup. Suppt. pl./12, fig. $1-2$.

Hesp. de Milo, id.
Envergure, 25 mill. et souvent moins.

- Ailes d'un brun noirâtre, plus ou En mai. moins saupoudrées de blanchâtre; supé- Clienille pubescente, mides des bois, mais on rieures avec beaucoup de taches blan- d'un brun grisatre ou le rencontre aussi dans ches grandcs el bien marquies; celles qui jaunfitre, avec une ligne les lieux secs. Il varic sont au-dessus de la tache intracellulaire dorsale plus foncée ou beaucoup.

au nombre de deux ou trois et toujours ferrugineuse et deux li- La question de savoir trés-visibles; celles de la rangie anté- gneslatérales d'un jaune si c'est à cetle espèce - marginale bien marquées aussi, quoique clair, ombrées de rous- ou au Fritillum que un peu plus incertaines que les autres; sâtre de chaque côté du doit être rapporté le $\boldsymbol{P}$. enfin la sous-cellulaire, auprès de la corps. Tête noire avec Malva de Linné, est base, toujours bien visible et souvent des poils isolés. Vit en trés-controversée. Dalgéminéc; inféricures à peine sinuées avril surle fraisier (Fra-man assure positiveprès de l'angle anal, avec deux rangs garia vesca) ment (pag. 202) qu'au-
de taches semblables, dont l'anté-mar-
cune espece voisine la base. France et un point al chetéc et rayée de bleû́- n'habite la Suède, et en la base. Frange nettement et fortement treet ponetuée de noir. entrecoupéc. Dessous des inférieures d'un gris olivatre, arec les nerrures plus claires, le bord abdominal entierement d'un gris obscur et des taches blanches; la supéricure des trois qui sont à la base plus petite ou du moins ne dépassant jamais les autres; l'intermédiaire presque toujours plus grande; celles du milieu de l'aile formant jusqu'it moitié une bande continue, puis un ou deux petits points arrondis, et celles de la rangic anté-terminale punctiformes, mais variant de taille et souvent oblitérćes en partie. Massue des antennes d'un ferrugineux foncé ou brun.

○ Sernblable.

Les taches blanches du milieu des ailes supéricures sont conluentes, et forment ainsi une large bande blanche transverse. En outre, celles de la série llexueuse sont allongées et terminces en coin intérieurement. Aux ailes inféricures la rangée anté-terminale est toujours bien marquée, mais la précédente est ordinairement réduite à un trait blanc, et on voit à la côte un gros point de même couleur. Le dessous se ressent plus ou moins de ces modifications.

Plus grand ( 28 mill.). - Les taches blanches sont grandes et aussi prononcées que dans les Alveolus les micux marqués. Aux ailes supérieures clles sont pour la plupart un peu arrondies; la sous-cellulaire est géminéc. Aux inférieures la rangée médiane est trismarquéc, et les deux taches qui suirent celles du bout de la cellule sont bien risibles et rectangulaires. Le dessous des supéricures ne differe point de celui d'Alvcolus, les taches y rederiennent quadrangulaires. Quant au dessous des inféricures, les dessins y sont presque complétement oblitérés et remplacés par une suance blanchatre, la tache du bout de la cellule est réunic avec celle marginale qui est vis-a-vis et la basilaire, de
Mêmes localités. conclut que l'slveolus cst très-certainement l'espèce que Linné a décrite sous le nom de Malia, M. Boisdural a adopté l'ayis de Dalman. D'un autre côté Godart (Encyel. 784) donne en faveur de l'oraisons qui semblent fort plausibles et qui sont appuyées par M. Zincken-Sommer. EnGi M. Treitschke ne se prononce point et reste dans le doute. Cette question est donc encore et sera long-temps indécise.

Nous arons pris plusicurs fois dans nos environs cette variété, qui est plus ou moins prononcée. Il parait qu'elle se trouve fort souvent, car presque tous les auteur's en ont parlé.

Ile de Milo. En mai.

Nous arons sous les ycux l'unique individu de ce Syrichtus, qui a servi a M. Duponchel pour sa figure et sa description. Il est tellement endommagé que nous n'osons aflirmer qu'il se rapporte bien a Alecolus; mais il présente d'une manière evidente les caracteres d'une variété accidentelle, et nous ne pensons pas qu'il doire constituer une espèce. Le dessous des inféricures est loin d'être aussi marqué que dans pinion contraire des 


\begin{tabular}{|c|c|c|c|}
\hline & $\begin{array}{l}\text { sorte qu'elles figurent une bande trans- } \\
\text { verse blanchatre qui se prolonge jusqu'd } \\
\text { la base. }\end{array}$ & & $\mid \begin{array}{l}\text { la figure, et d'ailleurs } \\
\text { il est en si mauvais état } \\
\text { qu'on n'y remarque rien } \\
\text { de bien distinct. Ce- } \\
\text { pendant cet individu } \\
\text { nous semble se rappro- } \\
\text { cher davantage d'Alveo- } \\
\text { lus (qui comme on sait } \\
\text { varie beaucoup pour la } \\
\text { taille) que d'aucune au- } \\
\text { tre espéce. }\end{array}$ \\
\hline $\begin{array}{l}\text { Frrmiles. } \\
\text { Hub. } 464-465 . \\
\text { Alveolus. var. Ochs. } \\
\text { God. }\end{array}$ & $\begin{array}{l}\text { - Beaucoup plus grand qu'Aleolus } \\
\text { (5o mill.). - diles peu saupoudrées de } \\
\text { blanehatre; supérieures ayant les taches } \\
\text { du bout de la cellule, de la rangée trans- } \\
\text { rerse et la sous-cellulaire, trés-appa- } \\
\text { rentes, la rangée anté-terminale nulle } \\
\text { ou indiquée senlement par deux ou trois } \\
\text { espaces d'atomes blanchatres, légers, au } \\
\text { bord interne; inféricures avec la tache } \\
\text { seule du bout de la cellule bien sensible, } \\
\text { la rangée anté-terminale peu marquée. } \\
\text { Dessous des supéricures assez semblable } \\
\text { A Alveolus. Dessous des inférieures avec } \\
\text { les mêmes dessins, mais ayant le fond } \\
\text { d'un. rouge de brique clair, aussi pro- } \\
\text { noncé que dans les individus clairs de } \\
\text { Sao. } \\
\text { q Semblable. }\end{array}$ & & $\begin{array}{l}\text { Bien que nous ayons } \\
\text { sous les yeux une belle } \\
\text { paire de ce. Syrichtus, } \\
\text { parfaitement sembla- } \\
\text { bles a la figure d'Hub- } \\
\text { ner, notis hésiterions a } \\
\text { le rapporter à Alveolus, } \\
\text { sans l'autorité d'Och- } \\
\text { scnheimer, Treitschke } \\
\text { ct Godart. Il a quelques } \\
\text { rapports arec les Fritil- } \\
\text { lum de Hongrie, et, s'il } \\
\text { est constant dans ses } \\
\text { caractères, on aura crée } \\
\text { bien des espèces qui } \\
\text { sont moins tranchées } \\
\text { que cette variété. }\end{array}$ \\
\hline $\begin{array}{l}\text { PROTо. } \\
\text { Ochs, God Bdv. } \\
\text { Hub. 98-921. } \\
\text { Bdv. Icon. pl. } 46 . \text { fig. } \\
\text { 4-5. } \\
\text { Dup. Suppl. pl. } 42.65 . \\
7-8 \text {. }\end{array}$ & 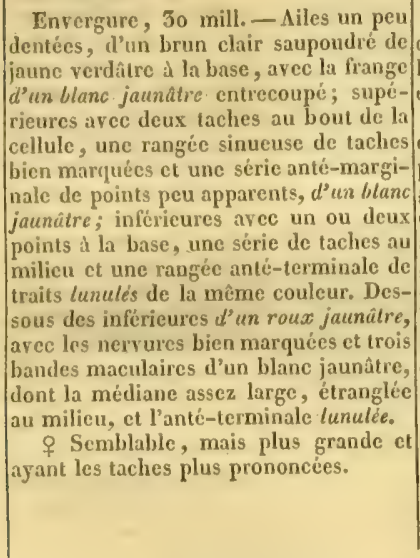 & $\begin{array}{l}\text { Espagne, Portugal, } \\
\text { environsde Montpellier, } \\
\text { II ongrie? Fin de juin } \\
\text { et courant de juillet. } \\
\text { Clienille pubescente, } \\
\text { d'un gris jaunâtre, à } \\
\text { premier anneau étran- } \\
\text { gle, marqué de deux ta- } \\
\text { ches d'un brun roux, et } \\
\text { ayant sur le dos une li- } \\
\text { gne et des atomes de } \\
\text { cette couleur. Poils } \\
\text { blancs, tête noire, pat- } \\
\text { tes jaunatres, stigmates } \\
\text { cerclés de brun. Vit en } \\
\text { mai sur le Phlomis lych- } \\
\text { nitis, dont elle lie les } \\
\text { feuilles avec de la soie. } \\
\text { Clirysalide allon- } \\
\text { gie, rougeatre, cou- } \\
\text { verte d'une poussière } \\
\text { blanche. }\end{array}$ & 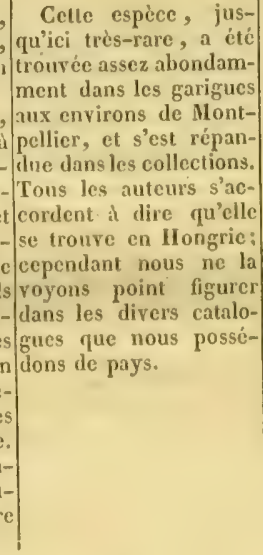 \\
\hline
\end{tabular}

(143) Ailes supérieures dépourvues de repli dans les deux sexes".

\begin{tabular}{|c|c|c|c|}
\hline $\begin{array}{l}\text { ORBIFER. } \\
\text { Treits. II ub. } 803-806 . \\
\text { Bdv. Icon. pl. 47. fig. } \\
\text { 1-2. } \\
\text { Dup. Suppl. pl. 42, fig. } \\
\text { 5-6. }\end{array}$ & $\begin{array}{l}\text { Envergure, } 29 \text { mill. - - Ailes entières, } \\
\text { d'un gris-brun foncé à reflet rougefitre, } \\
\text { arec la frange blanche entrecoupéc de } \\
\text { noir ct des taches blanches disposées } \\
\text { comme dans Sao. Dessons des inférieu- } \\
\text { res } d^{\prime} \text { 'un brun verdâtre ou jaunâtre, avec } \\
\text { troisscrics }\end{array}$ & $\begin{array}{l}\text { Iongrie, Morée, Dal- } \\
\text { matie, Italie. En mai } \\
\text { et juillet. }\end{array}$ & $\begin{array}{l}\text { Elle est maintenant } \\
\text { assez ripandue dans les } \\
\text { collections. }\end{array}$ \\
\hline
\end{tabular}

- Outie ce caractére, cette petite race se distinguera encore de la premiere $(1 / 4)$ en ce que les ailes inferieures sont generaleınent plus arrendies, un pü

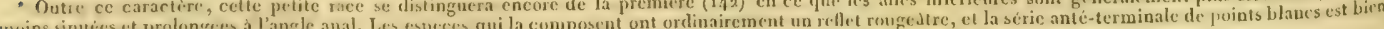
distiacte sur les quatre ailes, au lieu d'étre incertaine et formée d'atomes blanchatres comme dans les autres Syrichtus. 
Evcrate*

Ochs. God.

Dup. Suppl. pl. 4r. fig 7-8.

Orbifer. var. Treits. $10^{\circ}$ rol. p. 96 .

SAO.

IIub. $47 \mathrm{t}-472$. Bdv. God. pl. 28 B. fig. 3-4. Sertorius, Ochs. Le Tachete, Engr.

\section{mbur.}

THEAAPRE.

Bdv. Icon. pl. 46. fig. 6-7.

Dup. Suppl. pl. 42. fig. 9-10. |dont les plus grosses à la cóte et au bout de la cellule. Troisième entrecoupé de la frange des ailes supérieures égal aux autres. Collier et extrémité de l'anus d'un gris verdître. flet.

o Plus arrondie, plus píle et sans re-

Plus petite (2/f inill.), quelques taches grisâtres à la base des inféricures. Dessous des inférieures d'un rougeátre pâle, arec les taches disposées de même que dans Orbifer et également arrondies. Dessous de l'abdomen blanc, avec les bords et l'extrémité un peu rougeâtres.

Lnvergure, 24 mill. - Ailes entieres, d'un brun noir d reflet rougeâtre, avec Ia frange blanche entrecoupce de noir et arides. En mai et juillet des taches blanches; supérieures ayant la série anté-terminale composée de taches petites, arrondies, mais bien visibles; inférieures avec un trait discoïdal allongé, souvent maculaire, et une séric anté-terminale comme aux supéricures. Dessous de celles-ci semblable au dessus, mais plus clair et arec les taches plus grandes. Dessous des inférieures d'un rouge brique plus ou moins vit, avec trois rangées de taches blanches, dont l'intermédiaire composée de taches plus grandes et irregulieres. Troisieme entrecoupé blane de la frange des supérieures bcaucoup plus large que les autres. Collier et extrémité de l'anus rougeâtres.

o Semblable, mais d̀ ailes plus arrondies.

Differe de Sao, d'apres M. Rambur, par la série anté-marginale de taches

rapprochée du bord, par les taches discoidales des inféricures qui sont beaucoup plus larges, et enfin par la massuc des antennes, qui a la face interne d'un rouge foncé, tindis qu'elle est (dit-ilj noire dans $S$ aco.
Portugal.

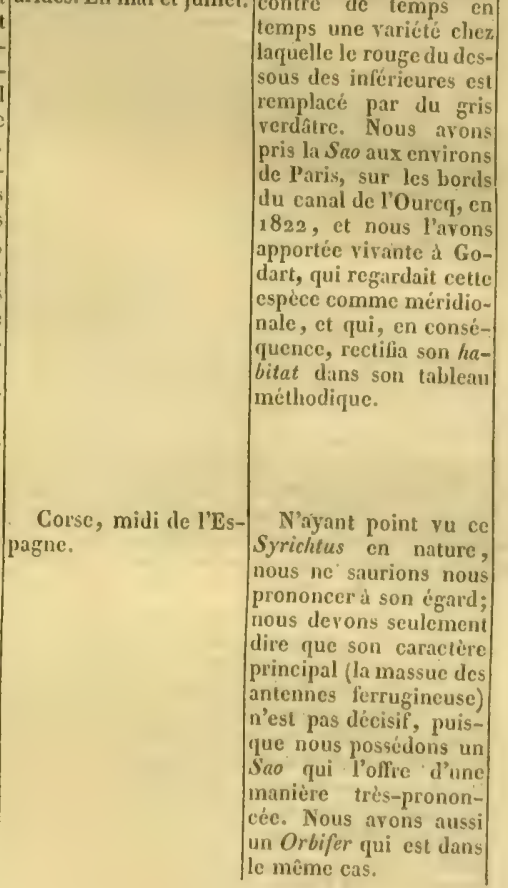

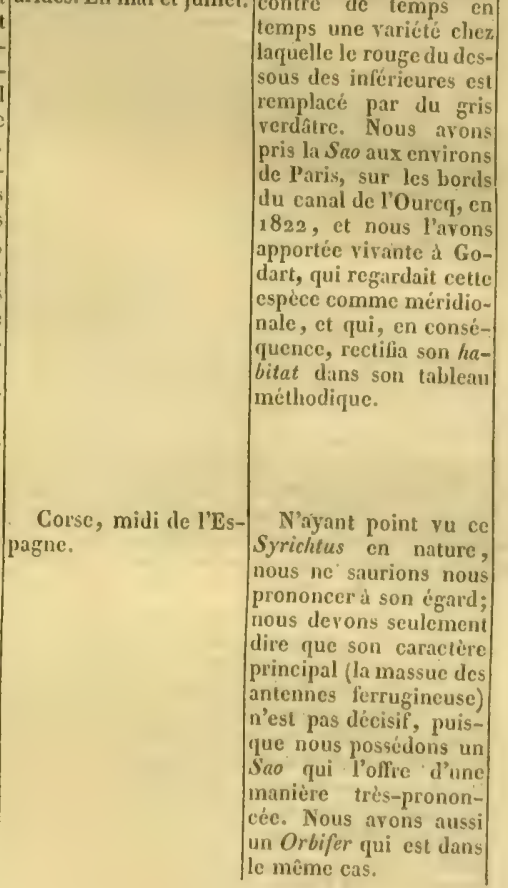

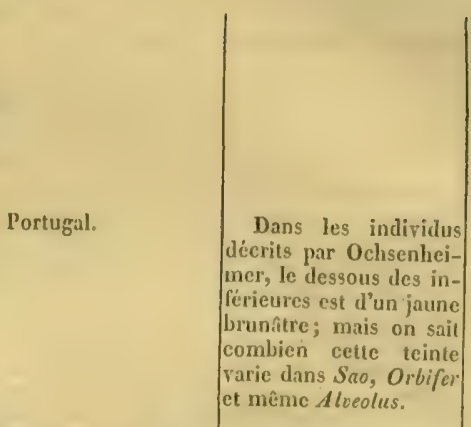

Dans une partic de Elle n'est nulle part Europe, lieux sees et(très-cummune. On rentemps une variété chez laquelle le rouge du dessous des inférieures est remplacé par du gris pris la $S_{\alpha o}$ aux environs de Paris, sur les bords du canal de l'Ource, en apportée virante \& Godart, qui regardait cette espece comme méridioquence, rectifia son habitat dans son tablean méthodique.

Syrichtus en nature,
nous ne saurions nous
prononcer à son égard;
nous devons seulement
dire que son caractère
principal (la massue des
anternes ferrugineuse)
n'est pas décisif, puis-
que nous possédons un
Sao qui l'offre d'une
nanière trés-pronon-
céc. Nous arons aussi
un Orbifer qui est dans
e même cas.

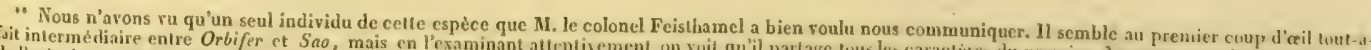

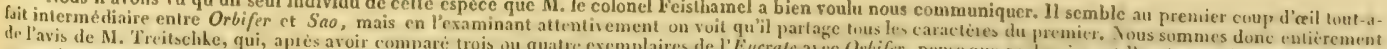

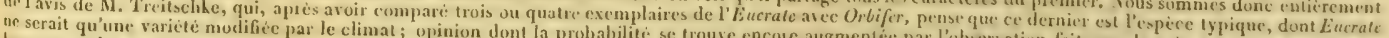

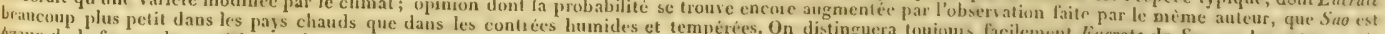

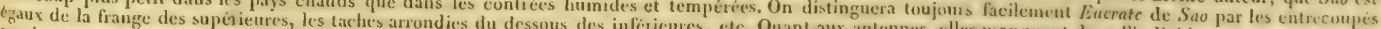

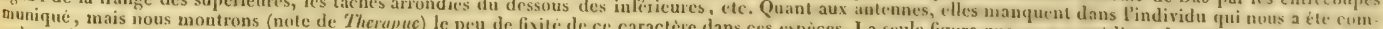
qu'en a dunné M. Duponchel, nous devons dire que l'enluminue ce caractère dans ces espéres. La seule figure que nons pussediuns de cette : spece itant eelle. ansi beaucoup moins arrondies et généralement moins grandes que dans la nature. 
MALV E.

Envergure, 29 mill.- - Ailes d'un gris| Dans une gronde par-| Il est très-commun, Fab. (non Lin.). Bdv. brun légèrement rougeâtre; supéricures lic de l'Europe. En må

God. pl. $12 \mathrm{sec}$. fig. 5 .

Hub. 45o-45z.

Malvarum. Ochs.

Le $\boldsymbol{P}$. Grisette. Engr.

ALTHEX

IIub. 452-453.

God. pl. 28 в b. Gg. 5-6. Hesp. de la Guimaute. légèrement dentées, as ec deux bandes et juillet. plus foncées; la première près de la Chenille pubescente, tivés. Il rarie un peu base, coudée sous la cellule, nettement couverte d'aspérités et pour les couleurs et coupée extérieurement, fondue inté- comme chagrinée, d'un inême pour la grandeur rieurement; la seconde flexueuse, in-gris cendré, avec deux des taches vitrées, mais certaine, interrompue, et éclairće cxté- lignes longitudinales ellesn'atteignent jamais ricurement d'une bande d'un gris ver-d'un gris plus clair de la taille et affectent bien dátre; les mêmes ailes ayant en outre chaque côté, et un col-rarement la forme de six petiles taches vitrées, dont trois réu- lier d'un jaune vif mar-celles de l'espèce suinies près de l'angle apical et les trois qué de deux taches noi- vante. Si cette espèce se autres groupées d̀ l'extrémité de la ccl-res. Tête noire et ru-trouve en Suéde, ce qui lule; inférieures très-fortement dentées, gueuse. Vit en juin et lest douteux, elle y est avec un point ì la base, une série mé- septembre sur différen-extrêmement rare et diane, puis une anté-terminale, de ta-tes espices de Mau-n'habite que les parties ches grisatres: le tout assez confus. Des-ves, enfermée dans une méridionales. Il est done sous plus clair que le dessus, plus uni, feuille qu'elle roule en certain que le $\boldsymbol{P}$. Matavec les taches des inféricures blanchî- cornet.

vae de Linné ne se raptres et plusapparentes, quoique plus ré- Chrysaliderenfermée porte point ici. Pour trécies. Massue des antennes étant in-dans cette feuille, d'un qu'il n'y ent pas de contéricurement d'un ferrugineux obscur et brun rougeâtre saupou-fusion, Ochsenheimer un peu courbée en crochet à l'extrémité. dré de bleuâtre. o Plus grainde, mais semblable. et quelques auteurs ont appelé celui-ci Malvarum; mais, comme on n'est point d'accord sur la véritable Malva, nous avons cru pouvoir laisser à celui-ci ce nom de Fabricius, sous lequel il est universellement connu en France.
Envergure, 32 mill. - Il ressemble beaucoup a Malva; et nous le ferons
micux connaître en l'y comparant. II est plus foncé; tout ce qui est d'un gris rougeâtre chez Malra est ici d'un gris verdâtre; la première bande noiratre est moins coudée, plus arrondie; les taches vitrées sont plus grandes: celles du disque sont droites intérieurement, lunulées extérieurement; les ailes inférieures sont presque noires, marquées au bout de la cellule de deux á trois taches blanchatres; en dessous elles ont au bord terminal de petits traits blancs longitudinaux qui s'avancent jusqu'au tiers de l'aile; enfin les supérieures ont en dessous, au bord interne, près de la base, un bouquet de poils d'un gris jaunatre ou verdatre fortement prononcé. La massue des antennes est droite, obtuse et non en crochet à l'extrémité, d'un noir profond, avec un point apical brun, visible seulement ì la loupe.

o Plus grande et un peu plus claire.
Centre de la France. En mai.
MII. Treitschke et Boisduval rapportent cette espèce di la précédente comme varieté Notre intention était d'abord de suivre leur exemple, mais un examen approfondi de ses caracteres nous en a empêchés. En eflet, indépendamment de ce que tous les individus que nous avons vus sont parfaitement semblables entre eux, et des différences constantes de conleur et de dessins qu'ils présentent, la forme des antennes et surtout le bouquet de poils dont nous parlons sont des différences d'organisation qui doivent sarement en amener d'équivalentes dans la chenille. Sa découverte, que nous n'avons pu faire, car l'espece est rare, prouvera si nous arons? 
Ochs. Bdv.

IIub. 454-455.

God. pl. 28 в b. fig. $7-8$. Alcere. Fab.

Engr. $9^{8 \text { E. D. }}$
Envergure, 34 mill. - Ailes d'un blanc jaunâtre; supéricures avec les mêmes rie, Alpes, Pyrénées, nous l'avons pris, e dessins que le précédent, mais d'un France méridionale, sieurs fois aux environs brun verdatre et les mêmes laches vitrées; inférieures dentées, avec deux let et aoat. bandes d'un brun verdatre, dessinant à la base un point, au milieu une bande continue, et près du bord une série de taches lunulées, aiguës, de la couleur du fond. Dessous des inférieures $d^{\prime} u n$ blanc jaunatre, sans taches, et sculement avec la transparence de celles du dessus.

q Plus grande ct parfois plus obscure. cu tort ou raison. Nous avons pris l'insecte parfait à La Rochelle en certaine quantité, et depuis, mais plus rarement, à Chartres, à Chatcaudun, et même une fois at laris, dans le bois de Boulogne.

(145) Ailes brunes, avec de petites taches ondies grisutres. - Frange culierc cl nullement cntrccoupéc. - Antennes comme dans la dixision 1 qo. - Un repli à la côte des ailes supéricures dans les máles.

Genre THANAOS. Bdv.

TAGES. $\quad$ Envergure, 27 mill. - Ailes très-en-| Dans une partic de| Cette cspèce est com-| Lin. Fab. Ochs. Bdv. tières, d'un brun clair, avec une série l'Europe. En avril, mai mune. Dans les indiviGod.

Hub. $456-457$. terminale de tres-petits points blancs; su- et juin. dus bien frais, l'extrèpérieures avec deux bandes plus fon- Chenille glabre, d'un mité apicale de la frange 12 sec. fir. God. pl. cées, éclairées de petites ondes blanchâ- vert pistache, avec deux des supéricures offre 12 sec. fig. 4. 4 tres, et dont l'interne maculaire et in-lignes latérales jaunes, une petite tache d'un Engr. Engr. $\quad$ culée des deux côtés et marquée de points noirs dont un doute un facies particutrois points blancs au sommet; inférieu- sur chaque anneau. Tête lier, mais avec un peu res ayant, outre la série terminale, un brune. Vit en mai et d'attention on y retroupoint discoildal et une rangée anté-ter- septembre sur l'Eryn- ve presque tous les caminale de points grisâtres. Dessous plus gium campestre et le Lo- ractères des Syrichtus clair, avec la série terminale bien appa-lus corniculatus. rente; inférieures ayant la série anté- Chrysalide verte, arec mours sont les mêmes. terminale et le point central également les anneaux de l'abdo-Les léécères diférences

MAILLYI.

Bdv, Icon. pl. 47. fig. yisibles.

Envergure, 29 mill. - Ailes très-enmen teintés de roussâ- qu'elle présente ne nous $\begin{array}{ll}\text { tre. } & \text { semblent done pas suf- }\end{array}$ semblent done pas suf-
fisantes pour constituer un genre séparé.

Morée. tières, d'un noir brun; supérieures mélangées, surtout vers l'extrémité, de poils d'un gris blanchatre, et traversées par deux bandes noires dont l'interne un peu maculaire et atteignant la côte et le bord interne, et l'externe gémince, dentée extérieurement et tcrminéc at l'angle apical par un empâtement noiratie et par deux ou trois points blanes; inférieures sans aucune tache. Dessous plus clair, arec un rang de cing ou six points correspondants a la liande giminéc du dessus et s'arrêtant au milicu de l'aile, et le sommet lavé de gris violâtre: inféricures sans taches. Antennes. noires, annelées de gris.
It n'existe de cette espèce qu'un seul individu pris en Mlorée par II. le docteur Marloy. D'après la figure et la description de M. Boisdural, il semble différer beaucoup de Tages, principalement parl'absence des points blanes marginaux: et M. Duponchel, qui l'a yu cu nature, assure, de son côté, qu'il constitue une espece séparce; nous arons done suivi ces deux autorités. No- 


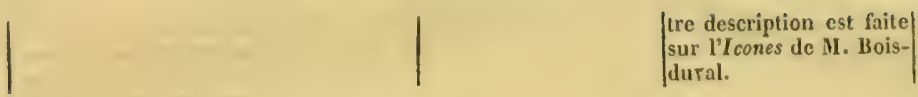

\title{
(146) Genre XIX. STEROPES (stÉropi).
}

\author{
(Bdv. Icon. des Chenilles.-Ueteropterus. Dup.)
}

Caractères principaux. - Chenille de tête saillante et a cou ctrangle. - Chrysalide longue, mince, munie d'une pointe assez longue a la parlic anterieure.- Ailes non parallèles dans le repos; les inférieures presque horizontales; les supdrieures verticales ou obliques. - Massue des antennes courte, renflec, presque droite et sans crochet à l'extrimité. - Abdomen plus lon" que les ailes inferieures. - Corps grêle ou peu robuste.-Point de repli a la côte des supérieures dans les deux sexes.

Caractires secondaires. - Ailes entieres, il frange peu ou point entrecoupie; les infirieures un peu prolongées, mais non sinuées a l'angle anal; fond des ailes d'un brun noir, avec des dessins jaunes.

Fab. Bdv.

ARACYNTHUS.

God. pl. 12 sec. et 12

tert. fig. 1.

Steropes, Ochs.

Hub. $475-474$.

Le Miroir. Engr.

pANISCUS.

Fab. Ochs, Bdy.

God. pl. 12 . fig. 1-2. Brontes. Iub. $47^{5}$ 476.

L'Echiquier. Engr.

Ochs. Iub. $477-478$. 641-644.
Envergure, 53 mill.-Ailes d'un brun| Centre de la France| Cette especec, si dif-| noir; les supérieures arce deux ou trois et de l'Europe, dans les férente des autres Hestaches jaunes près du sommet, et dont bois. Fin de juin et pérides de nos contrées, la plus grande est divisée en trois par les commencement de juil- n'est pas très-répandue. nervures et part de la cóte; les inféricu- let.

Nous l'ayons prise dans res larges, sans taches. Dessous des su- Chenille légèrement les elairières humides périeures brun, avec les taches du des-pubescente, d'un blanc de la forêt d'Hallate, sus et une ligne terminale courte, den- rerdatre, arecune ligne près de Pont-Saintetée intérieurement, d'un jaune vif. Des- obscure sur le vaisscau Maxence (Oise); on la sous des inférieures dı même jaune, dorsal et deux autres li- trouve aussi communéavec le bord abdominal brun et douze gnes latérales d'un blanc ment dans quelques lolar'ges taches ovales, d'un blanc jaunatre, jaunatre très-pale. Stig- calités des environs de cerclées de brun et contiguës. o Ayant la frange un peu entrecou- chagrinée, d'un brun La l'emelle est plus rare, pée, plus de taches jaunes sur les supé-- roux, avec une tache son vol est lourd, et on rieures et une série de taches grisatres/rousse sur le devant. est obligé de frapper les très-peu apparentes sur les inférieures. Pattes écailleuses rous-buissons pour la faire sâtres, membraneuses sortir.

de la couleur du fond.

Vit en mai et juin sur les graminées.

Chrysalide allongée,

d'un vert pâle, avce la

lête saillante et termi-

née par une pointe la-

vée de roussatite.

Envergure, 28 mill. - Ailes très-en- Nord et centre de l'Eutières, d'un brun noiratre, avec des taches d'un jaune fauve; celles des supéricures irrégulières et dont la série anté-terminale peu sensible; celles des inférieures arrondies et disposées, saroir: une près de la base, puis deux au- dos plus-foncé et deux l'ayons trouvéc, mais tres, dont la supérieure plus grande, lignes latérales jaunes. elle y est commune. La puis une série anté-terminale de six à Collier orangé, tête femelle vole plus tard scpt autres plus petites. Dessous des su. noire. Vit en avril sur le que le mâle, et ce n'est périeures jaune, avec des taches noires. plantain (Plantago ma-que vers quatre heures Dessous des inférieures d'un jaune sau- jor). poudré de brun, aveo les taches du dessus d'un brun plus clair et cerclées de noir.

$q$ Un peu plus pâle.

Ailes plus arrondies; supérieures en-

Allemagne, forêt Cette variété est bien du soir qu'on peut s'en procurer une certainc quantité. La chenille, que nous décrivons sur la figure d'Hubner, est peu connue, et cet iconograplie ne donne point la chrysalide.

èrement jaunes, ayant trois points al- d'Elm, duché de Bruns- constante et devra peutlongés à la base, un plus gros au bout de wick, Lironic, Russie être former uno ospèce; 
God. pl. 27 a a. fig. |la cellule, etune série anté-terminale de|méridionale, bords delmais on y retroure si $x-2$. pointsdont les intermédiaires plus petits l'Oural, En mai. l.c Jonquille. Engr. $\quad$ et plus marginaux, bruns; les inférieures sont scmblables à celles de Paniscus, mais le brun est saupoudré do jaune et les taches sont plus grandes. Dessous des supérieures semblable au dessus. Dessous des inférieures ayant la tache basilaire plus allongée. Antennes moins annelées et entièrement d'un jaune d'ocre en dessous.

+ Plus rapprochée de Paniscus et n'en diflérant qu'en ce que les taches jaunes des ailes supéricures sont conluentes en dessus.

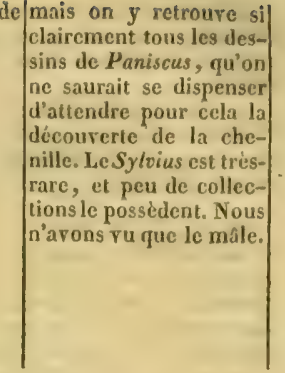

\section{(147) Genre XX. HESPERIA (Hespérie).}

(Latr. Ochs. God. Daim. etc.)

Caractères principaux. - Chenille de lete grosse et à premier anneau un peu étranglé. - Chrysalide allongée, mince, terminée antérieuremént par une pointe asscz courte, et ayant une gaine ventrale prolongée en un filet saillant. - Ailes superieures relecées dons le repos; inférieures horizontales ou obliques. - Antennes courtes, terminees en massue presque oroide, droile et souvent munie d'un petit crochet a l'extrémité. - Abdomen aussi long ou plus long que les ailes inférieures.-Corps robuste. -Point de repli a la côte des supérieures dans les deux sexes.

Caractires secondaires. - Frange non entrecoupie. - Ailes ordinairement jauncs; supericures ajant le plus souvent un trait noir discoidal, oblique, dans les mâles; inférieures sinuées près de l'angle anal. - Vol rapide d l'ardeur du soleil.

NOSTRADAMUS.

lab. God.
Bdr. Icon. plar; supérieures ayant la cóte et le Dup. Suppl. disque plus foncés; les inférieures ve4-6.

Pumilio. Ochs.

Pygmerus. 460.

COMMA.

Lin. Fab. Ochs. Bdv. God. pl. 12 tert. fig. 4

Hub. 479-481.

taches carrées, d'un jaune plus clair que r. le fond; supérieures très-aiguës au sompl. 45 . fig. 95 A. B. c D. G. II.

Dessous d'un cendré brunitre; supérieures ayant un peu de noir a la base

58- et une séric de taches jaunâtres peu sensibles; inférieures unicolores.

f Plus claire, ayant le disque des superieures marqué d'une série de petites taches d'un blanc jaunatre, la frange d'un gris clair et quelquefois le disque des inférieures jaunâtre.
Envergure 28 mill. - Ailes d'un jau- Dans toute l'Europe. ne faure, avec une large bordure brune En aoat. Chenille glabre, d'un ne se trouve jamais en vert-obscur mélangé de très - grande quantité ferrugineux, arec les dans nos environs. Les met, et ayant sur le disque un trait noir stigmates noirs, le col- bois sees et herbus, les ligne grise, trillante. Dessous des infé- de cette couleur au bas lées des parcs sont les rieures verdatre, avec deux séries de ta- des neuvième et dixiè-endroitsqu'elle fréquenches carrées, blanchätres et bordés de me anneaux. Tête bru- te de préférence. La finoir cxtérieurement. Antennes a massue ne. Pattes de la couleur gure d'Engramelle, très-globuleuse, arec un très-petit crocluet du corps. Vit en juillet quoique fort grossière, à l'extrémité.

f. Ayant les supérieures mnins aiguës au sommet et sans trait discoïdal.

SYIJVANUS

Fab. Ochs. Bdv.

Envergure 51 mill. - Ailes d'un fauve vif, avec une large bordure obscure et clairières des bois. En God. pl. 12 sec. fgg. 2 et une série anté-terminale de taches car-mai et juin. 12 tert. fig. 5. Hub. $482-484$. rées de la coulcur du fond; supérieures assez aiguës au sommet, et ayant sur le disque un gros trait noir, aigu aux deux extrémités. Dessous des inférieures d'un jaune verdâtrc, avec unc série de taches

\section{sur la Coronilla raria.}

Chrysalide brune.

nous semble se rappor-

ter ici plutôt qu'ì Sylvanus.

Elle est communc. On la rencontre surtout dans les endroits des bois un peu couverts et elle se pose de préférence surles feuilles; différant en cela de la précédente, qu préfíre les lieux ari- 
Ochs. Bdv.

ACTEON.

Hub. 488-490.

God. pl.27 $\Delta$ a. fig. 3-4. |unicolores, plus claires ot peu marquedes. Antennes a massue un peu allongde et munic a l'extrémité d'un crochet tressaillant.

\& Plus grande, plus rembrunie, à taches plus distinctes, avec les ailes supérieures plus arrondies et dépourvues de trait discoïdal.

Envergure 25 mill. - Ailes d'un brun-fauve clair; supérieures ayant la Hongrie, Autriche, cellule fauve, suivic d'une série courte et France, etc., etc. En

- Ilexuense de petites taches de la meme cou- juin et aoat. leur et soulignée d'un trait noir linéaire; inféricures sans taches. Dessous d'un gris jaunatre, tcinté de fauve à la côte des supérieures.

$q$ Dépouryue de trait noir discoidal et ayant parfois sur les inférieures quelques taches jaunâtres eflacées.

LINEA.

Fab. Ochs. Bdr. Hub. 485-487.

Envertrure 25 mill. - Ailes faures, arec une bordure trds-itroite et l'extré- En juillot, oot mité des nervures noire; supéricures tembre.

En juillet, aoot et sep-

II esp. Bande noire. ayant sous la cellule un trait noir linéai-
God. pl. 12 tert. re, continu, un peu courbe et assez long. God. pl. 12 tert. fig. 2.

La Bande noire. var. $9^{5}$ E. F. Engr.

VeNoLA. IIub. 666-669.

God.

Dup. Suppl. pl. 41. fig. $1=3$.

Buv. I con. pl. $47, \mathrm{gg}$ 4-5.

Virgula. Hub. 660-665 des antennes rousse on dessous. noir sur les supérieures.

N'en differe qu'en ce que le trait dis= coïdal noir manque, même chez le mâle.

Comme elle serapproche extrêmement Allemagne, France.

de Linea, et que tout le monde possède En juillet.

cette derniere, notre description sem Dessous des supéricures fauve, avec le crtendre, arec une li- engainantes delatige,

des et qui se pose surtout par terre ou sur les graminćes.

Elle n'est pascommune en France. Nous l'arons prise quelquelois aux environs de Chartres et de Cháteaudun; elle est moins rare dans le midi, et très-commune, dit-on, en Italie et en Dalmatie. Stes mours sont celles de la Commu.

Elle est très-commune; mais sa chenille est assez dillicile i trouver. Chenille glabre, d'un Elle vit dans les feuilles ert tendre, arec une li- engaînantes de la tige, et, sommet d'un gris jaunatre. Dessous des diviséę par un filet plus la tige même des gramiinféricures du même gris, arec le bord clair, puis deux lignes nées, dans les cndroits terminal faure; ces nuances bien tran-latérales d'un blanc jau-un peucouverts des bois cliées dans les individus frais. Massue nâtre. Tête et. pattes ou le long des murs des

o Un peu plus grande et sans trait bles à l'ocil nu. Vit en á élever. L'insecte paruin sur les graminées. fait vole dans les lieux Chrysalide d'un vert sees, et, dans nos conblanchâtre, avec la tête trées, il fréquente de saillante et terminće par préférence les champs une pointe courte. de céréales, qui servent peut-être aussi de nourriture it sa chenille.

A part le trait noir, la figure d'Hubnex présente bien tousles caractères de Linea; nous croyons donc que c'est ici, el non i Lincola, que cette variété dait se rapporter.

On a jusqu'ici trop confondu celte espece comparative. - La bordure noire du jaunâtre pâle, arec le puisse bien préciser son dessus des ailes est plus large, plus fon-vaisscau dorsal marqué habilat. On l'a d'abord due intérieurement; la frange est plus d'une ligne d'un blanc crue propre aux régions - claire; les supérieures sont moins aiguës jaunatre, et se prolon-montucuses du midi; au sommet, et leur trait noir est court, geant jusqu'd̀ la tête, mais nous l'arons prise droit, souvent interrompu et peu sensible. (qu'elle divise en deux; plusieurs fois auprés de Le dessous des supérieures est d'un fau-puis deux autres lignes Chateaudun, en plaine; ve presque uniforme, avec le sommet latérales de la nême ct M. Maillard, qui en a légèrement grisâtre; celui des inféricu-couleur, dont l'anté-décourert la chenille, res est d'un jaune un peu grisâtre, arec rieure en partie effacéell'a rencontrée près de le bord abdominal un peu plus jaune et sur les deux premiers Paris. Nous pensons plus clair; mais jamais ces nuances ne anneaux, et la posté- donc qu'en cherchant sont tranchées comme dans Linca. Les rieure bordantles pattes, attentivement eette esantennes ont la massue noire de part et.plus pale et à peine pèce on la trourera dans d'autre. 


\section{G. IIESPERIA.}

\& Plus pâle, arec les teintes grises du|tre. Vit en juin sur les/qu'habite $L$ inea, Il arridessous presque blanches.

graminées.

ve sourent que le trait

Chrysalide de même noir des supérieures

forme que Linea, arec manque, ce qui produit

le dos d'un vert-jaune une variétć analogue

marqué de trois lignes celle de l'espèce précé-

longitudinalesvertes. dente.

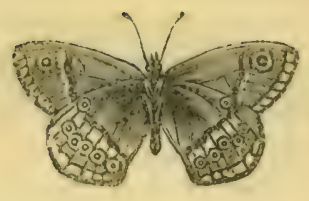




\title{
SUPPLÉMENT AU PREMIER VOLUME.
}

\section{PREMIERE PARTIE.}

\author{
Especes découvertes depuis la publication de nos premieres livraisons; additions, rectifications, descriptions \\ de chenilles ì visu *, etc., etc.
}

\section{PAGE 1, JGNE 6.}

\begin{abstract}
Aux caractères secondaires des Diurnes ajoutez celui-ci : - Ailes rivement el diversement cólorées en dessous.
\end{abstract}
Nors. Ce caraclère n'est pas tout-à-fait exclusif, il s'observe également chez quelques Nocturnes des tribus des Chélonides, Gatocalides et mềne cher ynelques Botys; mais, sauf ces rares exceptions (dans lesquelles mème il est pen prononcé), tons les Nocturnes, méme les plus brillants, ont le dessous des ailes de coulcurs sans éclat et unies, ou marquées de dessins rares et insignifiants.

\section{PAGE 1, LIGNE 21 .}

Le dernier caractère de la tribu des Ilespérides est exprimé d'une manière trop exclusive, le genre Syrichtus portant, dans le repos, les quatre ailes presque horizontalement. Voyez, pour les véritables caractères des IIlespérides, la page 214.

\section{Genre PAPILIO.}

$\left\{\begin{array}{l}\text { podalinus. } \\ \text { Piig. } 2 . \\ \text { Feistmanelit. } \\ \text { Pağ. } 5 .\end{array}\right.$
Nous ayons donné, it l'article de ces deux Lépidoptères, une description succincte de leurs chenilles; mais, une discussion s'étant élevée dans ces derniers temps sur la validité du second, considéré comme espèce, nous croyons faire plaisir à nos souscripteurs en leur donnant une description plus étendue des deux chenilles, afin de les mettre à portée de juger par eux-mêmes ce point d'histoire naturelle.

Nous arons sous les yeux deux chenilles de Podalirius, l'unc jeune, l'autre adulte; mais elle change tellement de livrée à ses différents ages, qu'elle est fort difficile à décrire. Voici d'abord les caractères qu'elle offre constamment et dans tous les périodes de son existence.

Elle est très-renflée antérieurement, atténuée postérieurement, complétement rase, mais d'un aspect velouté, d'un vert d'herbe gai qui jaunit à mesure qu'elle avance en age. Sur le vaisseau dorsal rìgne une ligne fine d'un jaune clair, et au-dessus des pattes on en voit une semblable un peu en relief qui fait le tour du cou, mais qui s'arrête d̀ la paire de pattes anale. Entre ces deux lignes on aperçoit une série de traits obliques du même jaune, mais moins prononcés, et qui sont plus ou moins nombreux et distincts, suivant l'âge de la chenille. Eñfin sur chaque anneau sont quatre points vésiculcux et disposés transversalement, jaunes dans le jeune age, orangés dans l’agge adulte, et qui palissent en approchant des derniers anneaux. Les pattes, vraies et fausses, et tout le dessous du corps, sont d'un rert blanchâtre très-pále. Les stigmates sont très-risibles dans le jeune âge, invisibles à l'oeil nu dans l'age adulte, d'un blane jaunâtre, cerclés finement de noir. La tête est verte et cachée en partie sous le premier anneau, comme dans tous les Papilio. Le tentacule en $\mathrm{Y}$ est jaunatre et transparent.

Indépendamment de ces caractères, qui, comme nous l'arons dit, ne varient pas, il existe sur cette chenille des taches d'un brun ferrugineux dont le nombre et l'intensité varient suivant l'âge. Dans la jeunesse on n'en voit allcune trace, mais dans l'age adulte clles se dessinent peu à peu. Nous prendrons pour les décrire un individu ayant subi sa dernière mue et huit d dix jours avant sa iransformation.

Les points ferrugineux sont disposés de chaque côté de la ligne dorsale ainsi qu'il suit : sur le premier anneau 1 , peu marqué, sur le second 2 , sur le troisième 3 , plus marqués et rangés obliquement, sur le quatrième 2 , mai leaucoup plus gros, surtout l'externe, rapprochés, raguement cernés de jaune et disposés en triangle avec le dernier point de l'anneau précédent. Chacun des segments suivants est marqué d'un point faisant suite au plus gros du quatrième, mais ils sont petits et diminuent de grosscur jusqu'au dixieme anneau, où ils deviennent très-grands et rä́uement cernés de jaune, après quoi ils s’arrêtent complétement. En outre les cinquième et sixième anneaux ont, au-dessus de la ligne latérale, chacun un point semblable oblong et géminé, et le quatrième en a aussi un, mais arrondi et situé au-dessous de cette lingne, qu'il interrompt sur cet anneau. Indépendamment de ces points, on en roit encore d'autres, mais peu apparents et d'un vert foncé ou brunâtre; ils ne deviennent bien sensibles que

\footnotetext{
- En terminant ce volame, nous répéterons une observation que nous avons déjá faite dans la préface. Mlalgré l'imporiance que nous attachons a la connaissance des chenilles et les recherches auxquelles nous nous livrons chaque jour pour les trouver, nous n’avons pu en observer par nous-mêmes dans les Diurne

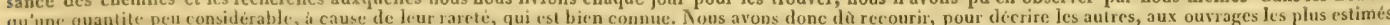

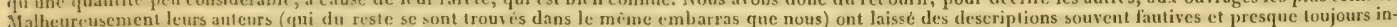

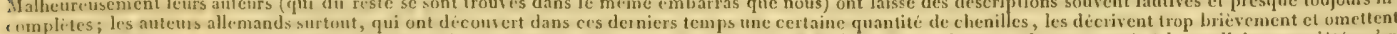
cemplites; les autetus allemands surtout, qui ont decousert dans ces derniers temps une certaine quantite de chenilles, les decrivent trop brievement el ometlent wharve dans les premiers ctats, varicti qui surpasse souvent celle des insectes parfaits; d'ailleus, àl'e pogue où beaucoup d'entre eux ont donné leurs figures, les arts de la gravure et de l'enluminure étaient trop peu avancés pour qu'on puisse asseoir avec cerlitude une description exacte sur leurs ouvrages.

Fous ne chercherous denc pas à disimuler a nos lectems que la scicnee a beateoup à faire sur ce point si important, et que plusieurs des descriptions que

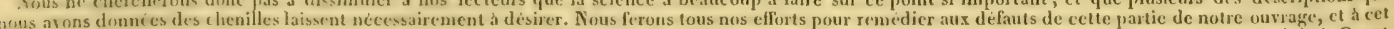
titet chacun des volumes suivants fresentera dans un petit supplement des rectifications successives. Deja nous en donnons quelques-unes dans celui-ci. Quaut aur. Bueturnes, teurs clenilles sont beaucoup micux connues, generalement moins difficiles a oblenir, et sous ce rapport nos volumes suivants laisseront beaucoup weins à désircr.
} 
frois ou quatie jours avant la transformation, et on en voit entre autres une série all-clessous. de la ligne laterrale. $\Lambda$ cette époque la couleur de la chenille a passé tout-d-fait au jaune sale.

Cette chenille est lourde, paresseuse, marche peu et imprime à son corps de temps à autre un bizarre mourement d'oscillation. Nous arons décrit page 2 sa chrysalide, qui est figurée dans notre planche (fig. 19).

La chenille de $F$ cisthamelii, d'aprés les dessins et les observations de M. Duponchel, est presque complétement semblable. Seulement les points orangés saillants n'existent pas; la ligne jaune latérale n'est point interromput sur le quatrieme annean, ou le point lemugineux latéral manque. Linfin les points dorsaux du dixième annean ue sont pas plus gros que les autres. Les chrysalides se ressemblent parfaitement.

Quelque minutieuses que soient ces différences, elles nous paraitraient sans doute suffisantes pour constituer une espèce si clles étaient constantes, celles que présente l'insecte parfait étant invariables sur tous les individus ohservés; mais ce point important n'est rien moins que prouvé. H. Duponchel n'a vu que deux chenilles du Feisthamelii; encore lui ont-elles été envoyées mortes et conservées dans l'esprit de vin, qui a da altérer leurs dessins et les rendre peu apparents, surtout les points orantrés, qui sont fort petits et dont la couleur est très-fugace; enfun, comme nous le disons plus haut, la chenille de Podalirius change considérablement ì ses diflérents îges, et elle oflre peut-être d'ailleurs des variétés. Il est done absolument indispensable, selon nous, arant de considérer ces deux Papilio comme distincts, qu'un bon observateur élève concurremment plusicurs chenilles des deux ot les examine scrupuleusement aux différentes époques de leur développement; ce qui n’est point difficile à réalises, les deux papitions se trourant, dit on, en Eispagne. En attendant nous derons laisser Fcisthamelii parmi les especes douteuses; puisque, a l'absence près des points orangés qui nous semble s'expliquer naturellement, les antediférences nous semblent du nombre de celles qui peuvent s'observer d'un individu i l'autre.

Pag. 4.

Aux caractères que nous arons donnés de cette variété il fiut ajouter celui d'aroir les bandes noires des iser. vures et celle transverse anté-terminale des premićres ailes plus épaisese et plus marquées en noir que dans les. Hachaon ordinaires. Ce caractére, du reste, est très-peu sensible sur la firgre d'Hubner; 'qui a créc celte prétendue espéce; mais il se trouve plus ou moins marque dans les individus que les amateurs sont convenus d'appeler Splryrus, et qu'on recoit le plus souvent du midi de l'Europe. C'est une variété très-peu remarquable et que nous ne décrivons que parce qu'elle a resu un nom séparé.

\section{Genre TIIAIS.}

HYPSIPYLE. CISSANDHA.

Pag. 4 et 5 . $P_{i g}, 5$.

Les descriptions que nous arons données des chenilles de ces deux Thais ont été faites, ainsi que nous l'arous dit, sur les figures d'Huloner, et nous n’avons séparé ces deux Lépidoptéres si voisins que d'aprés l'assertion de II. Boisduval, que leurs chenilles diflëraient constamment. Depuis, cet entomologiste lui-même nous a confirme ce fait; mais la figure de la chenille de Cassandra qu'il vient de publier difiere beaucoup de celle qu'a donnée Huliner. D'un autre côté, M. Treitschke insère dans son dixième volume un long article sur ces chenilles; il élève, dit-il, celle d'Hypsipyle tous les ans par centaines, et elle lui a offert une foule de variétés qui semblent analogues a celle qu'Hubner a figuríe sous le nom de Cassandra, et même à celle que M. Boisduval vient de fignter sous le même nom. De plus, il a vu éclore chez Dahl une quantité de chrysalides de Cassandra que ce marchand avait rapportées d'Italie, et il assure avoir trouvé parmi elles l'U ypsipyle ordinaire.

Ces épreuves trancheraient complétement la question s'il était bien démontré que les Cassandra ainsi obtenues sont identiques avec nos individus de France, et que les varietés de chenilles élevées par M. Treitsclike oflrent bien tous les caractères de celles de nos pays. Mais cette assertion suffit toujours, quant ì présent, pour que nous, donnions connaissance à nos lecteurs de cette divergence d'opinion entre deux entomologístes également recommandables. Il faut done attendre de nouveaux renscignements avant d'adopter un avis définitif, d'autant plus qu'il n'est pas certain que la Cassandra de M. Boisduval (qui est également la nôtre) soit bicn la mênze que celle de Dahl, puisque ectle derniere se trouve en Italic, pays que M. Boisduval assiarne pour patrie à l'Hypsipjle ordinaire. Nous engageons les amateurs a qui leur position le permet a comparer entre clles un grand nombre de chenilles de ces deux especes et à s'assurer qu'elles different constamment l'une de l'autre ainsi que leurs varictés. Ce ne sera qu'alors que la question sera définitivement résolue.

Nот. La Thais que M. Duponchel a figurée dans son supplément sous le nom de Cassandra est une variété de cette espéce dans laquelle la couleur jame est bien plus foncée et les taches rouges plus larges que dans les indi-
vidus ordinaires.

MEDESICASTE. Nous arons trouvé abondamment la chenille de celte espéce dans un voyage que nous arons fait cette année (1855) a Montpellier. Voici la description que nous en avons faite e.x visuet qui est un peu dif́rérente de celle que nous arons donnée d'aprés MI. Duponchel.

"Cheville jaunatre, avec quatre lignes noires interrompues, dont deux dorsales et deux latérales. Epines - fauves, cilićes de noir et plus foncées sur les premiers anneaux. Tête fauve, couverte de poils. Pattes écailleuses ") brunes, membraneuses jaunes; elle varie un peu pour la teinte du fond. Elle reste quelquefois deux ans en nchrysalide et est très-sujette dêtre piquéc par les Ichreumons. „)

\section{Genre DORITIS. Fob. Bdv.}

Plu.ieur's entomologristes nous ont exprimé le désir de nous voir adopter ce genre crúé par Fabricius et restreint par H. Boistuval a l'dpollinus des auteurs. Déja nous nous étions aperçs, en l'étudiant sur un individu en trés-maurais étal, que ce savant entomologiste n'avait pas minqué de raisons pour l'isoler; mais notre répugnance a multiplier les genres nous arait empêchés de l'adopter, par la raisun que les 
palpes different bien peu il l'œil nu de ceux des Parnassius (au moins de ceux de Mncmosyne), et que son facies le rapproche tout-i-fait de ce dernier genre; la tête, l'abdomen n'en différent pas non plus bien sensiblement. liestent done l'absenee de la poche cornée ct la forme des antennes qui le rapprochent des Thais, parmi lesquelles plusieurs auteur's l'ont placé, mais dont il difïre complétement par les palpes et l'habitus général. Ayant eu depuis à notre disposition des individus mienx conserrés, nous nous sommes convaincus de lit stabilité de ces caractéres, et nous pensons maintenant avec Ma. Boisduval et Duponchel, qu'ils sont suffisants pour constituer un genre séparé, vu la difficulté de rapporter l'A pollinus d'une manière bien satisfaisante à l'un ou à l'autre des genres roisins. Espérons, d'ailleurs, que la découverte de la chenille, que nous avions cru prudent d'attendre, viendra confirmer les différences que présente l'insecte parfait.

Voici done les caractères des deux genres Dorilis et Parnassius, caractères qu'il faudra substituer a ceux que nous arons donnés et adopter dans l'ordre suivant.

\section{Genre III. DORITIS (DoRitis).}

(Fab. Ochs. Bdv. Dup. (Tab. Méth.). - Thais. Latr. God.)

Cinractires priucipaux. - Chenille ........ - Antennes torminces par une massue allongete et sinuée. - Palpes tris-zelus, ne dépassant pas la touffe de poils qui garnit le front, $d$ arlicles indistincts. - Point de poche cornée snus l'abdomen des femelles.

Ciaractives secondaires. - Taille moyenne; les quatre ailes cnlicres, pen courertes d'écailles, gauffrces ct ridées transversalement; les infiricures non dentées au bord terminal, mais non arrondies el coupées un peu carrément; dessous luisant.

Oeserivation. Il u'est pas encore bien prouvé que ce gene se reproduise en Europe. Nous avions dit qu'il se trouvait en Morée, mais 'est d'aprés 11. Duponchel, qui avait été mal renseigné. La véritable patrie de l'A pollinus est l'Asie mineure, d'ot̀ proviennent presque tous ceux qui sont dans les collections. Cependant on dit qu'il habite aussi la Calabre et l'ile de Naxos.

\section{Genre III bis. PARNASSiUS (parnassien).}

(Latr. God. Bdv. Dup. - Doritis. Fab. Ochs.)

Ciaracteres principaux. - Chenille pubescentc. - Chrysalide arrondic, saupoudrée d'une poussierc bleuatre, attachie comme toutes celles des $P_{a-}$ pillonides, mais renfermée dans un leger réscau. - Antennes courtes, terminées par une massue slroite, grosse et presque ovoide. - Palpes courts, très-velus, dépassant a peine le toupet frontal, d articles assez distincts. - Abdomen tres-zelu dans les mâtes, et pourcu dans les femelles d'une poche cornée d son extrémitc.

Cirraclires secondaires. - Taille grande ou au-dessous de la moyenne, - Les quatre ailes entiires, arrondies; les superieurcs dipourzues d'icailles au bord marginal; dessous luisant mais uni.

Noт4. Supprimer la division et commencer à Apollo.

Il arrive sourent que les espèces de ce genre sont presque entièrement saupoudríes de noir. On observe fréquemment cette variété chez Apollo et Phobus, mais plus rarement chez Mnemosyne.

\section{Genre PIERIS.}
$\left\{\begin{array}{l}\text { Engaxe. } \\ \text { Pag. } 9 \\ \text { Freyer. Treits. }\end{array}\right.$
Y'ig. 11 .
DAPLIDICE. Pag. 11.
Bellidice.

\begin{abstract}
Vienne, et que Dahl, qui l'a prise en abondance en Dalmatic, l'a envoyée sous le même nom. Enfin M. Freyer l'a figuréc avec cette dénomination. On ignore ce qui a engagé Ilubner à la changer.

Cetle Piéride, qui constituc peut-être une espèce distincte, vole en mai et juin en Dalmatie, auprès de Rağuse, et en Italie, près de Florence.

Cette espèce forme avec Callidice, Chloridice et quelques espèces exotiques, un petit groupe très-naturel et bien distinct par le facies des autres Piérides marbrées de vert en dessous. Cependant ces. caracteres sont trop légers pour pouvoir fonder un genre séparé.

Cette jolie Pieride, d'ailleurs bien constante dans ses caracteres, n'est bien certainement qu'une varieté de Daplicide, et, ce qui le proure jusqu'd l'évidence, c'est qu'elles ont été élevées de la même chenille. Nous l'arons reçue de IIongrie, où elle parait commune. On nous l'a également envoyée de Suisse, mais plus grande que nos individus et ccux de Ifongrie.
\end{abstract}
Il paraît que cette Piéride est connue depuis long-temps sous le nom de Narcaca dans le Musée impérial de
M. Marchand possède une belle variété de Daplidice qui est entièrement d'un beau jaune-serin en dessus.

Genre ANTHOCHARIS. Bdv.

M. Boiscluval (dans son Iconographic des Chenilles, et dans l'Histoire naturelle des Lépidopteres du nord de la France de MI. Cantener) donne pour caractire a ec genre "une tache aurore au sonmet des ailes supéricures dans les miles, et les anneaux abdominaux de lit chrysalide immoliles et i peine visibles». Ces caracteres nous ont paru trop légers pour alopter ce renre, et d'ailleurs il nous semblait difficile de le distinguer de la Pier. Tagis, qui presente le second caractere sans offir le premier. Depuis, nous avons trouvé les chenilles des l'ier. Belia 
et Ausonia, et leurs elirysilicles nous ont irzilement offert la même particulariti. Il est même vraisemblable qu'clle existe aussi dins les autres Piéricles de cette division (Glauce, Belemia, cte.). Sur l'observation que nons en fimes à MI. Boisduval. il nous répondit que a toutes

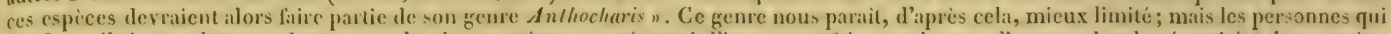

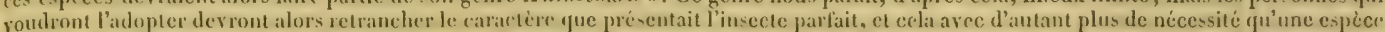
nouvellement découverte (Pier. Pyrothe Frersm.) semble faire la transition des espèces i sommet orangé (Cardamines, ete.) aux espices it ajles supérieures à sommet aiğts et sans tache orangée (Belia, Ausonia, ete,.).

Pour nous, ce genre, mêtue atec cettr extrusion, ne nous parait pas d’une absolue nécessité, les chenilles et leur nourriture ue différant point sensiblement de celles des autres l'iérites, et les mours de toutes ces espéces étant en général les mêmes. Nous nous contenterons done de changer les divisions de notre genre Pieris, \& partir de Callidice, ainsi qu'il suit:

Nous conservons la dirision (25).

Chrysalides de la même forme que celles de la division 22 bis. - Ailes inférieures des femelles plus ou moins mar-
quées de noir à lenr bord terminal.

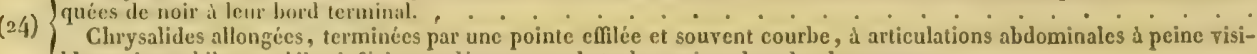

(bles et immobiles. - Ailes inférieures dépourvues de taches noires dans les deux sexes. . . . . . . . . . (25 bis)

(25) Chrysalides de la mime forme que celles de la dirision 22 bis. - Ailes inférisures des femelles plus ou moins marquées de noir a laur bort terminal.

\section{Callidice, Daplidice, Bellidice, Culubidice.}

(25 bis) Cheysalides allongies, dirticulations abdominales a peinc visibles ct immobiles. - Ailes infiricurcs dépournues de laches noires dans les deux sexes.

Genre ANTHOCHARIS. Bdr. . . . . . . . . . . . (26)

(26) Point de lache aurore au sommet des supérieures dans les mâles. . . . . . . . . . . (a6 bis)

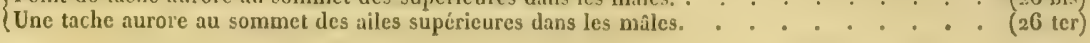

Point de tache aurore au sommet des supérieures dans tes mâles.

Belemia, Glavce, Belia, Thgrs, dusonia, Manchande.

Une tache aurore au sommet des ailes supcrieurcs dans les mâlcs.

Genre ANTHOCHARIS. Dup. Cant.

Pxnothor, Ciabanines, Euphemb, Euphexo.

BELIA.

Pag. 12.
AUSONIA.

Pug. 15.

Simplonia. $\left\{\begin{array}{l}\text { MaRCMANDEX } \\ \text { Pag. } 13\end{array}\right.$
Nous avons trouvé sa chenille cette annéc aux environs de Montpellier. Voici sa description : chenille d'un vert jaunâtre, couverte de petits tubercules pilifères noirs, avec trois lignes longitudinales d'un bleu riolâtre, dont une dorsale et les deux autres latérales, ces dernierres suivies d'une ligne blanchâtre. Pattes vertes. Tête verte, couverte de petits tubercules comme le corps, mais plus courts.

Elle vit en mai sur la Biscutella Didyma. Chrysalide d'un blanc-jaunâtre carné, arec la pointe antérieure trèslongue, légèrement arquée en dedans, d'un gris obscur; une ligne dorsale de la même couleur, accompagnéc de chaque cóté do très-petits points, et un trait pareil sur le hord de l'enveloppe des ailes; celle-ci peu saillante, striéc de gris clair, avec un point médian et une série anté-terminale d'autres plus petits, noirs.

Nora. Le papillon que nous a produit cette chenille se rapproche un peu de notre Var. $\mathbf{A}$. par la forme de ses taches nacrées, et tous les $B$ clia que nous avons pris pendant les mois de mai et de juin partagent plus ou moins ce caractère, mais la tache costale est semblable à celle des Belia ordinaires. Cependant, d'après le témoignage des naturalistes du midi, la véritable Belia vole en mars, et nous arons pu chez M. Adrien Devilliers un dessin de sa chenille qui differe de celle que nous arons élevée en ce que la ligne dorsale est jaune. Aurait-on confondu deux espèces sous le nom de Belia, on bien cette Piéride aurait-elle deux générations, l'une qui subirait toutes ses métamorphoses dans le courant de mai ct de juin, l'autre qui passerait l'hiver en chrysalide pour éclore au mois de mars? Nous appelons sur ce point l'attention des entomologistes du midi de la France.

Voici une description de la chenille qui nous a élé communiq̨uée par M. Germain, de Montpellicr.

Chenille d'un jaune-verdâtre, ayant une ligne dorsale violette, puis une ligne jaune, puis au-dessous une autre d'un vert-clair, enfin au-dessus des pattes un filct blanc bordé de jaune. Cette chenille est parsemée de tubercules piliferes violaties. Elle vit en mai sur la Biscutella Didyma.

Il serait peut-être plus juste de restituer à cette Piéride Ic nom de Marchande qu'Ilubner lui avait imposé arant tous les autres auteurs. 
PYROTHOE. Fiversm.

EUPIEME.

Esp.

Erothoe. Eversm.
Envergure, 35 mill. - Ailes blan- Russic méridionale. ches; supérieures aree la tache costalc Bords de l'Oural intélunulée et une tache apicale d'un rouge rieur. Ẻn arril. orangé enlouré de noir et découpant des taches marginales blanches. Dessous des mêmes ailes avec une ligne blanche dans la tache costale, et toute lat partic apicale noire du dessus colorie en vert, sans tache orangie ; inférieures du même rert avec beaucoup de taches blanches, dont trois oblongues et plus grandes. Antennes entitrement jaunatres.

Envergure, 45 mill. - Ailes $d^{\prime}$ 'un blanc jaunatre; supérieures avec la ta- Bords Volda bin che costale lunulée et une tache apicale transverse', itroite, d'un rouge orangé, saupoudrée al l'entour de noir et terminée à la côte par un espace de la couleur du fond. Dessous des mêmes ailes ayant le sommet jaune lavé cxtérieurement le blanc. Dessous des inférienres d'un jaune vif strié de noir à la côte, avec des taches blanches de diverses grandeurset très-irrégulières. Antennes blanches, cuisses roses.

o Ayant la lunule costale des supéricures plus grande et le sommet des mêmes ailes dépourvu de tache orangée et à peine teinté de cette coulcur.
Nous n'avons pas vu| cette espèce remarquable en nature, et notre description est faite sur le texte et les figures d'un mémoire inséré dans le recueil de la Société des Naturalistes de Moscow. Il n'y est point fait mention des sexes.

Nota. Gomme dans toutes les espèces roisines, la couleur du dessous des ailes se montre en transparence en dessus. Il en est de même de l'espèce suivante. - On placera celle-ci immédiatement avant Carlamines.

Russic méridionalc. Même observation que pour la précédente. Cette espéce semble se rapprocher un peud'Eupheno. M. Eversmann lui-même l'a reconnue dans l'Eupheme d'Ésper, dont la figure, dit-il, est très-mauvaise; mais, ainsi que l'observe fort justement M. Duponcluel, ce n’étnit pas une raisun pour lui donner un nouveau nom; nous lui avons done conservé le premier. Il faudra retrancher de notre article Eupheno tout ce qui est relatif à l'Eupheme d'Esper.

Celte espèce se placera entre Cardamines let Eupheno.

\section{Genre COLIAS.}

CinYsorumete. l'ate. 17.
On nous a reproché de ne regarder cette Coliade que comme une varicte. C'est done ici le cas de répéter ce que nous arons dit dans la préface : que les Lépidoptères renfermés dans une accolade avec d'autres et en plus petits caractères ne sont pas pour nous des zariètés, mais seulement des espèces clouteuses, Avant d'affirmer positivement qu'un Lépidoptère est variété d'un autre, il faut l'avoir obtenu de la même chenille, de mêmé qu'il faut avoir obtenu deux lépidopteres de chenilles différentes arant d'aflirmer qu'ils constituent deux espèces. Or, dans l'ignorance où on est des premiers états de la plupart des Diurnes, ce qu'il y a de plus sagre, suivant nous, est de rester dans le doute toutes les fois qu'une espece se rapjproche assez d'une autre pour le faire naître. Nous n'avions donc pas d'autre manière d'exprimer ce doute que de réunir prorisoirement dans la même accolade les espèces quí ne nous semblaient pas bien authentiques; et d'ailleurs, ainsi que nous le disons a l'article Chrysotheme, il est bien plus facile d'aperceroir les caractires qui séparent deux papillons par une description differencié que par deux descriptions complètes, quoique la première espèce soit bien plus difficile et prête beaucoup plus à la critique que la seconde. Nous espérons done que nos lecteurs nous sauront gré de cette marche, qui leur évitera des longueur's inutiles et qui d'ailleurs laisse dans son entier la question, que chacun peut alors décider suivant sa maničre de roir. 


\section{Genre POLYOMAIATUS.}

lag. $2 x$.

DORYLAS.

ANGUS.

Pag. 25.

C Prlaon.

risch.

Pag. 23.

EGON.

IIub.

Pag. 24.

BAVIUS.

liversm.

\section{Ortilete.}

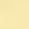

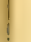


let jaunâtres aux ailes postérieures. $\Delta \mathrm{n}-$ tennes annelées, arec l'extrémité de la massue fauve.

BOETICUS.

Pag. 5 z.

Pag. 40.

Pag. 36

EVIPPUS parg. 41.

TRIANGULUM. l'ag. 54 . l'ag. 62 .

CIIAIUCI.EA, Pag. 73 .

MESIROPE.
La descriplion que nous arons donnéc de la chenille de ce Polyommate est incomplète. Eñ voici une plus circonstancié, faite d'après plusieurs individus adultes.

Elle est d'un rert plus ou moins terne, ou d'un rouge brun foneé. Sur le vaisseau dorsal se voit une ligne plus foncée, et au-dessus des paltes une baude d'un vert site ou d'un jaune lrunitue. mais, dans tous le's cas, plus pâle: que le fond. $A u$-dessus se roit une sietic de traits obliques, doubles, de la même coulerur, séparcis par un petit espace de la couleur du fond. Enfin, au-dessous sont les stigmates, qui sont gros, bien visibles et blanchitres. I.e demier anneau est marqué de quelques points de celte derniere couleur. Le dessous du corps est de la couleur de la bande latérale. La tête est pelite, noire ou d'un roux chair.

La chrysalide est d'un gris roussitre plus on moins obscur, pointilléc de noir, arec deux rangies dorsales de points noirs et les jointures des anneaux plus claires.

Elle vit, comme nous l'avous dit, dans les siliques du Baguenaudier, où on la trouve sourent en abondance. pour se unctre en chrysalide elle perce la silique, mais celles qu'on élive en captivité se métamorphosent quelquefois sans en sortir. On reconnait facilement sa présence en mettant la silique entre l'oil et la lumiere. On ţa for alors facilement dans le bas une masse noire produite par l'accumulation de ses exeríments. Si la grousse est percéc d'un trou non fermé avec de la soic, on peut être certain que la chenille l'a abandonnée.

Nous avons reçu plusieurs individus de ce Polyommate, et nous l'arons pris nous-même de nouveau cette anne (1855). Les caractires qui le séparent du Lymceas sont bien constants, et de plus, les femelles n'ont point ordinarement de taches faures sur les supérieures. Il ent done trés-vraisemblable que l'Essuli doit former une capéce distincte. Nous espérons que nos correspondants du midi de la France nous procureront bientôt sa chenille, ce qui nous mettra en état de décider la question.

Voici une description de sa chenille qui nous a été communiquée par M. Germain, de Montpellier :

"Chenille tris-déprime dans sa partic posterieure, d'un lirun sombre, arec une raic dorsalc noire bordéc de chaque cóté par des nunnces inégales de couleur jaune, peu apparentes,"

Nous regrettons que cetle deseription soit si courle et si peu circonstanciese, surtout a canse de lit forme particuliere de l'insecte parlait, yui en amene peut-être une correspondante daus la chenille. Nous engrageons les amiteurs du midi de la Erance à l'élever de noureau et à en donner une bonne figure et une description exacte, ainsi que de la chrysalide, ce qui n’a pas été fait jusqqu'ici.

Il arrire souvent que la chenille est dépourvue des points jaunes qui entrecoupent les traits obliques latéraux.

\section{Genre VANESSA.}

M. Germain, de Montpellier, nous a également communiqué la description suivante de la chenille :

Elle est d'un bleu clair rayé transreralement de jaune, surtout sur les quatre premiers anneaux. Chaque anneau est rarni de epe épines velues, jatnes it la base, brunsitres à l'extrémité; celles du dos sont moins longrues que les autres, et a cúté se trourent deux points d'un noir hleu luisant, visibles seulement à patir du quatrieme anneau, et entre lesrquels la couleur du fond est d'un brun clair. La tête est d'un rouge faure, surmontée de deux épines courtes et aiguës.

Elle vit en mai sur la pariétairc (Parietaria officinalis).

\section{Genre ARGYNNIS.}

Nous arons dit d l'article de cette Argynne que nous présumions qu'elle n'était autre que celle dont on a voulu faire une espèce dans ces derniers temps sous le nom de Boisduralii". Depuis, nous avons été confirmés dans notrc foring

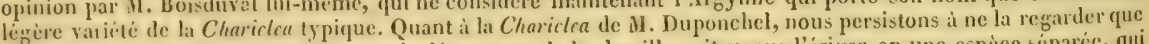
comme variété de Palr's, jusqu'i ce que la découverte de la chenille soit venue l'ériorer en une espéce séparce, qui alors devrait recevoir un noureau nom.

Ia Chariclea est toujours très-rare dlans les collections.

\section{Genre MELITAA.}

M. Auderreg, de Gamsen, ient de découvrir la chenille de cette petite Mélitée si semblable i l'Arlemis. Il a envoyé cette chenille ì 11 . Boistuval, qui a élé a même de se conyaincre qu'elle est complélement différente de celle d'Arlemis, puisqu'elle présente de larges taches jaunes sur un fond noir, tandis que la dernière est, comme nous

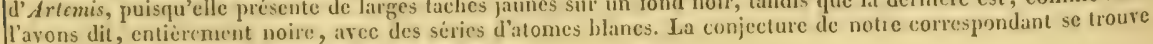

- Il s'eat glissé dans cet article, page 63, ligne 1ze, une faute tgpegraphique assez grave; au licu de : touchant la question, il faut lire : tranchant la question. 
done vérifiée, et l'on devra désormais considérer, avec MM. Trcitschke et de Prunner, la Melitaa Merope comme lune espece bien distincte.

\section{Genre SATYRUS.}

PIHLEA

Pag. 112.

Nous avons regu de Suisse une variété de ce Satyre chez les deux sexes de laquelle le disque est d'un fauve qui tranche fortement sur la bordure, ce qui lui donne la plus grande ressemblance en dessus arec Arcanius, dont elle ne se distingue absolument que par la taille. Mais on la reconnaitra facilement par le dessous des inféricures, où les yeux sont plus petits, mieux alignés, et la ligne médiane plus régulièrement dentée, sans”anfractuosité plus forte au bout de la cellule, enfin par ses antennes, dont la massue est noiratre en dessus et blanchítre en dessous, au lieu d'être rousse inf̦érieurement comme chez Arcanius. Cette variété est assez remarquable et differe beaucoup de nos Philea de France.

Noти. Chez la femelle de $\boldsymbol{P}$ hilea le petit œil apical reparait quelquefois en dessous; il en est de même chez cectte variété.

\section{Genre SYRICITUS.}

FRITILLUM Pag. 216.
Nous ayons pris cette année plusieurs indiridus d'une variété cliez laquelle le jaune rerdâtre est remplacé par dı blanc légèrement jaunatre comme chez Carthami, mais qui présente tous les autres caractères de Fritillum. 


\section{SUPPLÉMENT.}

\section{SEGONDE PARTIE.}

\section{Eispices itrangères (suivent nous) ì l'Europe, mais qui ont ilé donnés comme curopicmes par plusicurs auteurs*.}

\section{Genre PAPILIO.}

$\Lambda \mathrm{JAX}$.

Smith-Abboth. Bdy. Lépid. de l'Amérique. pl. 1, fig. 1-4. Ochs.

Dup. Suppl. p. 11 514.

XUTIIUS.

Lin. Fab. Cram. God. Bdv. Icon. pl. 1. fig. 1-2
Envergure, 75 mill. - Ailes d'un brun| noir, avec des bandes d'un jaune clair, Virrinie. En mers, mo dont la médiane bifurquée par en haut, et juin.

l'anté-terminale ondulée ; inférieure arec deux lunules bleues anté-termina-deux lignes latérales cherches qu'on a faites les et une tache anale noire, marquée d'un blanc verdâtre et pour le trouver en Liud'un trait bleu et surmontées de deux une bande transverse rope ayant été sans régrosses taches d'un rouge vif. Dessous sur le quatrième an-sultat, MM. Treitschke, des inférieures avec une ligne médiane neau, composée de trois Duponchel, Boisduval, rouge accolée d̀ une ligne blanche.

$$
\text { \& Semblable. }
$$

Envergure, $9^{5}$ mill. - Ailes noires Chine, Perse, Thibet, arec des taches et des raies d'un jaune Sibérie, Russic asiatisoufré; supéricures en ayant deux au que. bout de la cellule en forme de croissant et figurant une sorte d'œil; inférieures ayant une série anté-terminale de taches lunulées surmontées chacune d'un groupe d'atomes d'un gris jaunatre, angle anal marqué d'un oil jaune pupillé de noir et surmonté d'une tache bleue.

o Semblable, mais plus grande. couleurs, bleu foncé, etc., ctc., l'ont rejeté bleu clair et jaune vif; avec raison du catalogue tentacules de cette der-de nos Lépidoptères, nièrecouleur; stigmates dont il differe compléroussatres; tête et pat- tement par son facies. tes yertes. Vit en arril ( 11 formerait le $n^{\circ} 1$ et seplembre sur le Por- du genre Papilio.) celia Pygmaa.

Chrysalide ferrugineuse, arec des lignes plus claires.

Pespece n'a cncuropéenne que par M. Boisuluval, qui considere les insectes de la Sibéric comme indigènes. Nous ne saurions partager cet avis, quelque estime que nous professions pour cet entomologiste; et quand même le Pap. Xuthus ne s'éloignerait pas par son facies des especes de nos contrées, nous attendrions pour l'admettre parmi elles qu'il ait ćté pris plusieurs fois et qu’il soit prouvé qu'il se reproduit en deca des limites que les géographes assignent à l'Europe.

(II se placerait après
jax.)

- Indejendamment des espices qui vont suivre, il en est d'autres sur la patrie desquclies on n'a pas encore toute la certilude désiralıle, cl qu'un facies équiso-

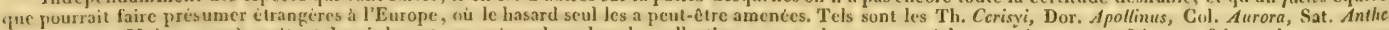
fea, Mc. etc. Mlais ces espèces itant depuis long-temps répandues dans les collections, nous n'avons pas osé les supprimer; toutefois nous faisons des roux pour que les observalcurs à qui leur position le permet elevent leurs chenilles et s'assurent qu'ils se reproduisent annuellement dans les contrées européennes. 


\section{Genre PARNASSIUS.}

Handwickir.

Tab. Synopt.

lag. 7 .
Il n'est pas bien certain que la variété femelle de Phobus, que nous avons décrite sous ce nom et qu'on nous a communiquéc comme venant de Suisse, soit le véritable Parn. Hardwichii de M. IIope. Comme nous n'avons point vu en nature les individus sur lesquels cet entomologiste a établi cette espèce et qui n'habitent point l'Europe, il est possible qu'ils offrent des différences sensibles d'avec celui que nous avons décrit. Nos souscripteur: devront done attendre cette comparaison avant de ranger deffinitivement ce Parnassien au nombre des Lépiclop-
tères européens.

\section{Genre PIERIS.}

CHEiRAN'TII. God. Hub. 6/77-648.

Fab. God. Ochs.

Dup. Suppl. pl. 5. fig. $1-2$.

Esp.?

II llica. Ilub. Lép. cxot
Un peu plus grande que la P. Brassica, avee laquelle elle a les plus grands rapports. Fond des ailes d'une teinte plus jaunatre, au moing dans les femelles; taches noires beaucoup plus dilatées et envahissant une partie des ailes supéricures; celle du bord interne se joignant fréquemment is la liture du mème bord. Dessous plus vif en couleur.

Envergurc, 48 mill.-Ailes blanches; les supéricures un peu aiguës au sommet, où elles sont marquées d'une large tache noire divisée par de gros points blancs, les mêmes ailes marquées d̀ la côte d'une grosse tache noire; inférieures arec quelques points noirs à l'extrémité des nervures. Dessous de celle-ci blane, arec les nervures légèrement dessinées en gris noirâtre, decoupant des lunules marginales blanches, et saupoudré gà et lit, mais surtout à la base et au milieu, de jaune d'ocre.

q Ayant une tache noire au bord interne des supéricures, et le bard marginal des inférieures noir et divisé par des laches blanches comme chez Daplidice.

\section{Ténériffe.}

Peut-être n'est-clle| qu'une modification locale de notre Pier. Brassica. (Elle se placerait immédiatement avant clle).

Cap de Bonnc-Espérance.

Elle fait partie du même groupe que $D a-$ plidice et Callidice, mais elle est certainement étrangère à l'Europe. (Elle se placerait immédiatement arant $\mathrm{Cal}$ lidice).

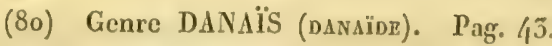

(Katr. God. Bdy. Dup. - Euplexu. Fab. Ochs.)

Cinractères principaux. - Chenille rase, munic de prolongernents charnus ou dpines molles et filiformes. - Chrysalicle grosse, courte, non anguleuse et marquée de taches métalliques. - Antennes longues, d massue grossissant insensiblement. - Palpes tricsecartés, dépassant peu la tele, lcur secont article d peine une fois plus long que le précédent. - Cellule discoildale des
secondes ailes fermée.

Caractires secondaires. - Abulomen grele, assez long. - Ailes larges, sinuées; les infirieures ajant, dans les mûles, une petite poche sous la cellule discoillale. Des points blancs sur la lete, le corselet, la poitrine et la bordure des ailes.

CIIIYSIPIUS,

Lin. Fab. Ochs. Hub. $678 \cdot 679$.

Bdv. Icon. pl. 18 , fig. 3. Chrysippe. God. Encycl. Dup. Suppl. pl. 17. fig. $:-2$.
Envergure, 75 mill. - Niles d'un fauve . Indes orientales, Syroux, avee une bordure brune marquéc rie, Egypte.

de points blanes; supérieures sinuées Chenille d'un blan dans les contrées que au bord terminal, avec le faure plus violatre, ayee blanc nous cilons. Le hasard foncé le long de la côte, et la porduci-layant amené quelques très-ćlargie à l'angle apical, où elle est sions noires 'et des an- indiviclus sur les côtes même con-d'Italie, l'espèce s'y est marqués de jaune propagée pendant decix naux, d'une bande blanche divisée en dans leur milieu; épi- années (1806 et 1807), taches par les nervures, et de quelques nes au nombre de six, mais en 1808 elle a dispoints blancs; inférieures d'un fiuve deux sur le cou, deux paru complétement et uniforme, avec la bordure étroite, trois sur le cinquième an-ne s'y est pas montréc points noiratres suirant la cellule, et lalneau ct deux sur le on-lepuis. 
poche de la même couleur. Dessous zième. Tête de la coupresque semblable, mais ayant le som-leur du corps et rayée met des supéricures faure.

o Semblable, mais dépourrue de la clepias. poche des ailes inférieures.

Chrysa lide' d'univert clair, arec une ligne noire sur l'abdomen et des points argentés.

\section{ALCIPPUS}

Fial Ochs. cédente, dont elle ne differe du reste Bdv, Icon. pl. 18. 6g. 4. que par les ailes infërieures, qui sont Alcippe. Goul Encycl. blanches de part et d'autre, arec un Dup. Suppl. pl. $17 \mathrm{fg}$. I peu de faure sur les bords. 5.

Même localités.

Nous ne connaissons point sa chenille, mais II. Boisduval dit qu'elle differe constamment de celle de la précédente.

\section{Genre SATYRUS.}

\begin{tabular}{l|r|r} 
DARGETI. Envergure, $48 \mathrm{mill}$ - - Ailes un peu & Syrie,
\end{tabular}

I.efebv. Ann. de la Soc. arrondies, blanches, arec urre bordure
Entom. Dup. Suppl. pl. 26. 6g. nales de la couleur du fond; supérieu5-6.

res ayaut la base de la cellule cntiere-

Titea. Klug. Emprich ment blanche, et au-dela de son milieu un et Elrenb. Symb. petit point grêle se confondant presque phys. déc. $5^{\circ}$. pl. 29. avec la tache annulaire, qui est très-irréfig. $15-18$.

ALSO.

Vdv. Icon. pl. gulière, non évidée au centre et saupoudrée à l'entour de grisâtre; un petit cil bleuâtre au sommet de l'aile; inféricures ombrées de noir à la base, mais peu largement et a peine jusqu'd la ligne basilaire; leur bordure marquce de trois il quatre yeux pupillés de bleu. Dessous des supérieures ayant la tache annulaire très-petite et bien évidée. Dessous des inférieures arec les lignes basilaire et médiane comme dans Herta. Antennes rousses au somroet.

o Ayant les yeux micux marqués, surtout en dessous.

Envergure, 50 mill. - Ailes minces, un peu transparentes, d'un grisátre sale mêlé de jaunitre, avec queliques atomes bruns plus denses près de la frange; supérieures ayant une ombre légère sur le disque; inféricures laissant apercevoir en transparence les dessins du dessons, qui est brunâtre jusq̨u'ì la ligne médiane, arec des atomes grisatres, puis d'un gris blanchâtre un peu violacé et légèrement strié de noirâtre; frange grisâtse entrecoupéc de noiratre. Antennes comme dans les analomues.

I 9 Inconnuc.
.

Sibérie.
Malgré les rapports évidents que présente ce Satyre avec les espèces d'Lurope, et quoiqu'il soit le seul exotique qu'on connaisse dans cette section (Arge, Boisduval). II nous semble indispensable d'at tendre, pour l'admettre parmi les européens, qu'on l'ait trouvé plusieurs fois dans les limites de cette partie du monde. (La place de ce Satyre serait entre $\boldsymbol{H e r}$ ta et Clotho.)

Nous ne l'arons point ru en nature, et notre description est faite d'aprìs Al. Boisdural. Son habitat dit assez qu'il ne saurait être admis parmi les espèces européennes. (II se placerait apres $A$ Bno.)
$7 / y$ se coscors

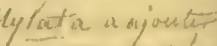

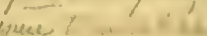




\section{DES TERMES DONT NOUS NOUS SERVONS DANS CE VOLUME.}

\section{AVERTISSEM ENT.}

Au lieu de donner une simple explication de la planche qui accompagne ce premier volune, nous avons pensé qu'il serait plus utile pour les commençants de leur donner une analyse complete des parties qui composent les Lépidopteres sous leurs troiy états. C'ést ce quis: nous allons faire ci-aprìs; mais comme cette analyse n'oflrirait pas, pour les recherches précipitées, toute la commodité d'un dictionnaire, nous disposerons à la suite, par ordre alphabétique, tous les termes que nous allons expliquer par ordre de matières, avec l'inslication de la page oit on en trouvera l'explication. On n'aura done qu'à se reporter à cette page oú chuque terme, étant imprimé en italique, fiapper. facilement les yeux.

Nous devons également prévenir que tout ce que nous allons dire ri-après ne s'applique qu'aux Diurnes. Chacun des volumes suivants contiendra les généralités relatives aux familles qui y seront traitées.

Un Lépidoptere ou Papillon passe par quatre états bien distincts : celui d'auf, celui de chenille, celui de chrysalide et celui d'insecte parail. Les phénomènes du premier état lui étant communs arec une foule d'animaux des classes supérieures et ne présentant rien de pårticulier, nous pas ¿ rons de suite d̀ l'état de chenille.

\section{CHAPITRE $I^{\text {er }}$.}

ÉTAT dE chenille (fig. $16,17,18$ ).

C'est le seul, des trois états qui nous restent à examiner, ou l'insecte prenne une nourriture bien substanticlle ct où il soit susceptible d'acquérir un développement extérieur, en un mot de grandir. Ce développement est pour ainsi dire de deux sortes : celui qu'il acquieit en assimilant les substances qui le nourrissent et qui lui est commun avec les autres animaux, et celui auquel il parvient par des mues on changements de penu successifs. C'est dans le jeune âge et peu aprés la sortie de l'œuf que ces changements de peau sont les plus fréquents ct les plus rapprochés.

Parvenue à l'age adulte, la chenille présente extéricurement les parties suivantes:

Lá têle (a. fig. 16 et 17 ) est composée de deux calottes de consistance cornée, dont les faces latérales se nomment joues et sont marquées de petits points saillants, souvent noirs, dont on ignore l'usage. $\Lambda$ sa partic inférieure se voit la bouche, dans la composition de láquelle entrent deux mandibules, deux mâchoires et une livre au milieu de laquelle est un bouton percé d'un petit trou qu'on nomme filiir et qui est destiné à donner passage à un liquide qui se durcit it l'air et forme la soie dont lit chenille se sert pour filer sa coque ou se suspendre aux branches, Chacune des mâchoires porte un palpe, et la lèvre elle-même en a deux.

Le corps (a. d. fig. 16) est composé d'une suite d'articulations au nombre de douze et qu'on nomme segments ou anneaux. La partie par laquelle ces anneaux se touchent se nomme jointure ou incision. Le corps est long, le plus sourent cylindrique, parfois aplati en dessous; d'autres fois court, convexe en dessus et atténué aux extrémités. La chenille est dite alor's onisciforme, à cause de sa ressemblance avec les cloportes (oniscus). On dirise le corps en trois parties, le dos, les côtés et le ventre.

Sur le dos court un filet longitudinal, souvent transparent et laissant apercevoir le vaisseau dorsal. Sur les côtés se voient des ourertures en forme de boutonnieres, bordées d'un petit bourrelet saillant et qu'on nomme stigmates. Ces stigmates sont au nombre de neuf seillement de chaque côté, les deuxième, troisième et douzième anneaux en étant toujours dépourvus; ils correspondent ì autant de petils vaisseaux ou trachees par lesquels la chenille respire. A l'cxtrémité du dernier anneau est un autre orifice auquel aboutit le canal digestif. et qui est l'anus. Le dos et les côtés sont ornés de couleurs et de dessins qui varient d̀ l'infini.

Le ventre est souvent aplati, couvert d'une peau plus fine que celle du dessus de l'insecte, jamais velu ni epineux. Il est rare qu'on y observe des dessins, et ses couleurs sont généralement pâles et uniformes. Il est toujours garni d'appendices servant à la progression et qu'on nomme pattes.

Ces paltes sont de deux sortes : celles qui garnissent les trois premiers anneaux sont invariablement au nombre de six; elles sont de consistance cornée, se terminent en pointe et s'appellent pattes dcailleuses ou rrairs pattes (b. b. b. fig. 16), parce que ce sont les seules qui doivent reparaitre dans l'insecte parfait. Les autres (c. c. c. c. c. fig. 16), qui doivent s'eflacer complétement par la suite, ont une tout autre forme; elles sont grosses, molles, cylindriques et terminées par une suite de petits crochets disposés circulairement et formant ce qu'on appelle la couronne. Ces dernieres pattes, qu'on nomme membraneuses ou fausses pattes, sont toujours dans les Diurnes au nombre de dix; elles sont disposées par paires sur les septième, huitième, neuvieme, dixième et douziène anneaux, et la dernière paire s'appelle
anale.

Telles sont les parties extérieures de la chenille. Son anatomie intéricure sort tout-i-fait de notre cadre, et nous ne nous en occuperons pas. Nous allons examiner maintenant les différents vêtemeots et appendices des chenilles, et nous dirnns un mot ensuite sur leurs dessina et leurs couleurs.

Quand le corps de la chenille est complétement dépourru de poils, il est dit ras ou glabre (Sat. Pamphilus, etc.); s’il est chargé de poils très-courts et serrés, on l'appelle pubescent ( Polyommatus, etc.); si ces poils sont plus longs, ils est telu. Les poils sont disposés sur le corps de plusieurs manieres; ainsi, ils sont droits ou couchés, implantés directement sur la peau ou sur des tubcrcules plus ou moins gros. 
Ces tubercules s'appellent alors pilifires (Picris, Colias, ete.). Souvent un tubercule porte une aigrette de poils rangés circulairement on verticilles (Hamearis).

Outre les poils, les chenilles sont souvent chargées d'appendices de formes variées; tantôt ce sont de petites granulations fines et serrées, et alors on dit que la chenille est chagrinie ou rugueuse; tantôt les tubercules sont moins nombreux et affectent la forme de mameloss allongés (Lim., Camilla, Populi) : d'autres fois ils sont coniques et couverts de poils ou ciliés (Melitaa); souvent enfin ils constituent de véritables ćpincs, qui sont elles-mêmes ciliées (Argynnis), ou branchues (Vanessa); plus rarcnent ces épines sont grlabres, molles et flexibles (Danais). La tête elle-même porte sourent plusieurs de ces appendices. Fréquemment elle est granulée et hispide (Van. Atalanta), quelquefois ornée de deux épines divergentes (Van. Prorsa, $C$. album). Si ces épines sont fortes et allongées, elles prennent le nom de cornes (Apatura) et sont quelquefois au nombre de quatre (Charaxes).

La partie postérieure on anale de la chenille est elle-même parfois accompagnée d'appendices ou terminée d'une manière particuliere ; plus souvent elle est arrondie (Argynnis, etc.), mais d'autres fois elle est échancrée (Charaxes); plus souvent encore elle offre deux pointes qu'on nomme pointes caudales (Satyrus).

Indépendamment des appendices que nous venons de décrire, les chenilles en présentent une foule d'autres; mais ils sont tous le partage exclusif des nocturnes, et nous n'arons pas it nous en occuper ici.

Les couleurs des chenilles sont trés-variées; le vert est cependant la plus répandue. Les dessins ne subissent pas moins de modifications; les figures qqui les composent (points, taches, bandes, etc.) scront expliquées au chapitre III. Nous nous bornerons donc ici à expliquer les directions qui sont spéciales aux chenilles.

Une ligne ou une bande est dorsale, latcrale, ventrale, anale, suivant qu'elle est placéc sur l'une ou l'autre de ces parties. Elle est dite lon. gitudinale quand clle se dirige de la tête à l'anus (fig. 16 i d), transverse quand elle est parallèle aux incisions, oblique quand elle s'écarte de l'une ou de l'autre de ces directions.

Les chenilles des Diurnes vivent exclusivement de plantes, et beaucoup affectionnent chacune leur nourriture particulière. Quand elles mangent indistinctement tout ce qu'on leur donne on les appelle Polyphages; mais c'est le plus petit nombre. Presque toutes fuient la lumière et la chaleur, et se retirent pendant le jour sous les pierres, la mousse, les herbes, les écorces, etc., etc.Quelques-unes se reneontrent facilement, mais la majeure partie est rare, quoique souvent les insectes parfaits snient très-répandus. Les innombrables variations de forme qu'clles affectent suivant les races offrent pour la classification des caractères souvent plus sars que ceux de leurs papillons; aussi leus connaissance constitue-t-elle seule le véritable entomologiste. C'est d'ailleurs al ce premier état que la nature a attaché le plus,d'intérêt, en variant a l'infini leurs manières de vivre, les ruses par lesquelles elles échappent à leurs nombreux ennemis, les moyens qu'elles emploient pour se soustraire à l'influence des saisons contraires et les conséquences de leur adresse et de leur admirable prèvoyance. Nous devons donc recommander vivement aux jeunes amateurs l'étude de ces ingénieux animaux, seule source encore fíconde d'importantes décourertes et de plaisirs sang cesse renouvelés.

\section{CIIAPITRE II.}

ÉTAT DE CMRYsAXIDE (fig. $19 \cdot 20,21)$.

Quand la chenille a acquis tout le développement dont clle est susceptible, elle se dispose à subir sa transformation. Pour cela elle cesse de prendre de la nourriture, cherche une place commode, s'y suspend de la maniere qui lui est propre, on file sa coque quand elle doit en avoir une. Ces préliminaires terminés, ses couleurs se salissent, ses dessins s'oblitèrent, son corps se contracte et se raccourcit; enfin elle change de peau une dernière fois, et des-lors elle est changée en chrysalide.

Sous cette forme l'insecte ne prend aucune nourriture, et son état habituel est une complète immobilité. Il vit cependant et se prépare peu d p̉eu à subir une métamorphose plus étonnante encore que la première.

La chrysalide se compose de deux parties bien distinctes qui ont recu les noms d'antérieure el de postérieure; la dernière ne comprend que l'étui de l'abdomen : elle est composéc d’anneaux portant latéralement des stigmates comme ceux de la chenille, et se termine en une pointe conique.

La partie antérieure comprend les étuis de la tête, du thorax et des ailes. On y distingue facilement les enreloppes des pattes et des aniennes, qui sont appliquées longitudinalement sous la poitrine, entre l'enveloppe des ailes, qui est toujours bien marquée. Enfin l'étui do thorax se voit sur le dos et se prolonge jusqu'aux anneaux de la partic postérieure.

La lorme des chrysalides varic beaucoup : clles sont anguleuses (fig. 19) quand elles présentent, dans une partic queleonque, des angles aigus, obtuses quand ces angles sont émoussés (fig. 21), arrondies quand elles n'offrent aucune espèce d'angles (Parn. Appollo). Cette derniere forme est pres(yue exclusivement le partage des Nocturnes. Les angles principaux se voient sur la tête (Papilio, Pieris) et sur le thorax (Satyrus, Limenitis); si cette partie est longitudinalement taillée en coin, elle est dite caréne.

Les chrysalides offrent quelquefois, comme Ies chenilles, des poils et des appendices, mais plus rarement; des bosses sur le thorax ( $L i$ menitis, $V_{\text {ancssa) }}$, des pointes à la partic antéricure (Papilio), des séries de houtons sur le dos (Sat. Mara, Megara, Melit. Artemis, etc.), enfin quelqques petits poils courts et serrés (Polyom. W. Album), tels sont les principaux appendices des chrysalides dans les Diurues eưropéens.

Le mode de transformation est très-varié et fort important pour la classification. Souvent les chrysalides sont attachées par la queue et maintenues par un fil qui ceint le corps (Papillonides, fig. $19-21$ ). I a tête est alors presque toujours en haut, mais cette posture n'est pas

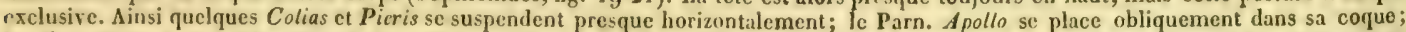
les Gonopteryx tournent la pointe de leur tête vers la terre, etc., etc. D'autres fois la chrysalide est suspendue seulement par la queue, et la tête est alors constamment dirigéc en bas (Nymphalides, fig. 20); enfin d'autres chrysalides sont roulées dans des feuilles (II esperia), ou posées sur la terre sans aucun licn (quelques Satyrus). Chez les nocturnes, Ic mode de transformation est encore plus varie.

Quant aux couleurs et aux dessins, ils sont en général peu remarquables chez les chrysalides. Cependant plusicurs offrent des taches dorćes ct argentées très-brillantes (Vanessa, Argynnis, Dunais, etc.).

Le temps que l'insecle passe à l'etat de chrysalide est nrdinairement, chez les diurnes, de quinze jours ou trois semaines. Aux approches de celte époque, celle-ci acquiert une couleur plus foncéc, puiselle devient transparente et luisse voir sur l'enveloppe des ailes uuc partic 

des dessins de l'insecte parfait; enfin celui-ci s'en digage d'abord mıu et humide; mais il se sèche et se raffermit i l'air en peu de temps,
et jouit dés lors de la faculté de voler et de se reproduire. C'est sous cet ćlat que nous allons maintenant l'étudier.

\title{
CHAPITRE III.
}

\author{
ÉTAT D'INSECTE RAMPAIT (fig. I et 14).
}

C'est ce chapitre que nous allons traiter le plus longuement, tant à cause de son importance qu'i cause de la facilité que présente l'insecte La vie du papillon est courie et dép position ne permet d'étudier l'entomologie que sur des collections.

sitôt qu'il a rempli ce but de la nature.

Les Diurnes volent tous en plein

ferent dans toute son ardeur.

Nous allons examiner successiyement toutes les parties du papillon.

\section{S Ie:. De la tele.}

La tête (fig. 2-3) est composée de plusieurs parties bien distinctes. Les yeux (d. fig. 2-5) sont arrondis, globuleux, très-saillants et taillés en une multitude innombrable de facettes destinées à suppléer à l'immobilité de ces organes. Ils varient pour la grosseur proportionnelle, et peurent servir à distinguer quelques races; mais ce caractère est en général peu distinctif parce qu'il ne présente rien d'exclusif.
$\mathrm{Au}$-dessus des yeux est le front (a. fir. 2-5), qui est touje

toupet frontal, et qui, dans la classification, 2-5), qui est toujours plus ou moins garni de poils; ces poils forment en avant ce qu'on appelle lisses ou stemmates; mais ils sont très-petits, difficiles a a relativement la longueur des palpes. $\Lambda$ sa partic supérieure sont situés les yeux

$\mathrm{Au}$-dessous des yeux sont placés les palpes *; ils se distingercevoir et ne s'observent que dans les Nocturnes.

ceptibles, tandis que les seconds sont très-développésetsont d'un palpes supérieurs et palpes inférieurs; mais les premiers sont ì peine persés de trois articles qui rarient de longueur et se s'aperçoit à peine; le second est plus long et habituellement plus ou moins facilement. Le premier est situé pour ainsi dire derrière l'œil et tus, droit ou courbe, nu ou poilu, etc., etc. La fig. 5 remént velu. Quant au dernier, il est tantót très-petit et tantôt prolongé, aigu ou ubon peut facilement compter les articles.

Entre les palpes est située la spiritro

monde connât l'usage de cet organe.

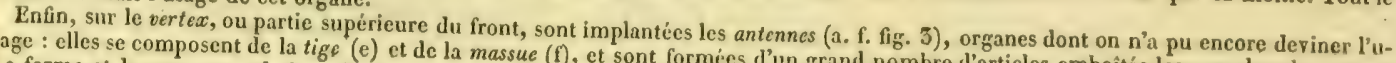
La forme et la grosseur relatives de la massue sont (₹), et sont formées d'un grand nombre d'articles emboités les uns dans les autres. quand elle forme un boutón plus ou moins détaché d'un grand usage dans la classification. On dit qu'elle est abrupte oudistincte de la tige

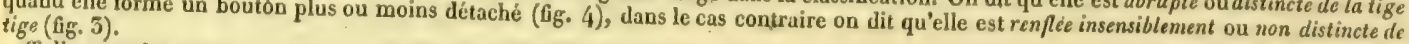
Telles sont les parties qui composent la tête. L'ensemble des palpes, de la trompe et de la partie sur laquelle cette dernière est située
se nomme la buuche.

\$II. Duthorax.

Le thorax est la partie qui suit la tête et qui porte les pattes et les ailes. Il est composé de trois pieces principales, dont l'antérieure se nornme prothorax ou collier, Ie médiane mésothorax, et la postérieure métathorax; ces deux dernières parties sont peu distinctes dans sculement ici deux des pièces du mésothorax, quiéces articulées dans le détail desquelles nous n'entrerons pas; nous mentionnerons (b. fig. 1).

Le dessous du thorax s'appelle la poitrine : clle porte des orranes essenticls, c'est-i-dire los patts (0 nombre de six, et se composent de plusicurs pie porte des organes essenticls, c'est-i-dire les pattes (fig. 7 ); ces pattes sont toujours au cuisse (b), qui est beaucoup plus longue et habituellovoir : la hanche (a. fig. 7 ), piece trés-courte qui attache la patte a la poitrine; la (f) dans les hespérides, et le tarse (d), et habituellement velue; la jambe (c), dont l'extrémité est arméc d'une paire d'er zols ou d'épincs Dans les nymphalides le tarse des deux pattes antérieures s'oblitère (fis. 8 ) terminé par un double crochet (c) serrant à la préhension. chets â l'extrémité; d'où il suit que cette paire de pattes s'oblitère (fig. 8) et se réduit à un scul article ordinairement velu et sans crop ttes ambulatoires; on les appelle abusirement Tetropodes peut ctre utile a la progression. On dit de ces insectes qu'ils n'ont que quatre dits Hexapodes.

Le thorax porte encore, comme nous l'arons dit, les ailes ; mais ces ormanes sont n'en traitions pas dans un paragraphe séparé:

\section{SIII. De l'abdomen.}

L'abdomen (c. fig. 1) est composé d'une suite d'anneaux comme la chenille et la chrysalide. C'est la seule partie dans laquelle on retrouve des traces du premier état de l'insecte. Il est pourvu, comme celles-ci, de stigmates lateraux, et se termine par une ouverture qui les femelles en une saillie cornée, rétractile ci consistent chez les mâles en deux valves latérales et l'organe mâle proprement dit; chez

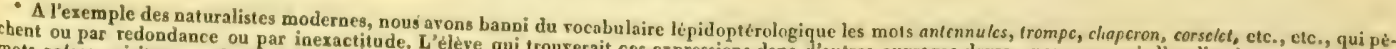

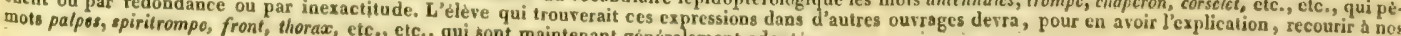
mots palpes, spiritrompo, front, thorax, etc., etc., qui bont maintenant généralement adoptés.
} 
L'aludomen est en général peu important pour les diagnoses spieifiques. Chez quelques genres (Thais Papilio) il est orné de bardes Jongitudinales et de points laterax ; dans d'autres, et c'est la plus grande partic, il est brun ou noir en-dessus, gris nu jaunitre en desnnus. A pris la mort de l'insecte il se desseche et se replie en dessous, au moins dans les mâles; car les femelles l'ont quelquefois si rempli l'œufs qu’il conserve alors sa forme primitive.

\section{SIV. Des ailes.}

Les ailes des Lépilopteres sont au nombre de quatre, formíes de deux membranes minces et transparentes exactement superposées, et recouvertes sur leur surface extéricure d'une infinité d'ćcrilles imbriquées, adhérentes à l'aile par un pédicule, et qui, à l'œil nu, ont l'asject d'une poussiere fne. Elles sont variables de forme et très-diverscment colorées et forment les dessins des ailes dont nous nous occuperons tout ì l'heure.

Les toublanes des ailes sont supnortées par une charpente qui les consolide et les vivifie. Cette charpente n'est autre chose que ce guron appelle les nercures : ce sone de petits canaux creux dans lesquels circule une liqueur incolore et dont nous allons détailler la disposition. La figure g représente deux des ailes d'un Lipidoptire du grenre Papilio. Tout ce que nous allons dire de ces deux ailes concerne inalement les deux autres, qui leur sont toujours exaciement semblables pour l'organisation.

La premiere (1) s'appelle aile supéricure ou anlérieure. Elle a presque toujours une forme subtriangulaire, et offe conséquemment trois angles principaux et trois côtés ou bords. Le premier angle (a) se nomme la base, c'est lui qui s'articule avec le thorax; le second (b) se nomme angle catcrne ou mieux angle apical; le troisieme (c) angle intsme. Le bord supéricur (a-b) a reru le nom de bord externe ou untiricur, mais surlout de cole; celui qui lui est opposé se nomme bord interne, intérieur on postcrieur (nous l'appelons toujours bord interne); cnfin le troisième (b-c) s'appelle bord extericur, et surtout bord terminal ou marginal.

La surface de l'aile a, en outre, recu d'autres noms, mais tout-i-fait de conrention puisqu'on n'y aperçoit aucunes limites. $\Lambda$ insi toute la partic de l'aile qui aroisine la base s'appelle elle-même base et donne le nom de basilaires aux dessins qu'elle supporte. L'angle apical indique une autre portion qui se nomme le sommet de l'aile et donne le nom d'apicaux aux dessins qui s'y trouvent; les figures qui longent la rite se nomme costales; tout le milieu de l'aile a recu le nom impropre de centre ou cclui plus convenable de disque, et communique aux dessins qu'on y aperçoit le nom de discoidaux ou cenlraux; enfin le bord terminal fit appeler terminaux ou marginaux les dessins qui le touchent.

(Duant aux nerrures, clles sont principales ou sccondaires. Les premières sont au nombre de trois (ou micux de quatre, voyez plus bas); elles partent de lit base de l'aile et se ramifient pour former les nervures secondaires ou nervules, dont le nombre varie un peu. I,es pre作 j'une autre nerrure souvent très-renfléc, mais qui la suit tellement qu'on a l'habitude de les considérer comme une seule et même nerrure; clle envoie i l'extrémité de l'aile plusicurs petites nervules peu importantes; la seconde (g) s'appelle médiane; elle s'écarte de la premicre, et poisse quatre ou cinq rameaux ou nerculcs. L'espace qu'elle laisse entre elle et la costale se nomme cellule (i), et la position

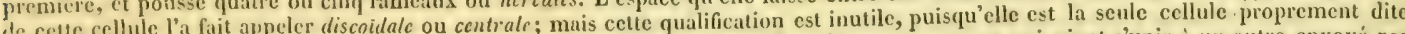
qu'on voie sur les ailes des Lépidoptéres. Cette cellule est souvent forméc (fig. 9) par un rameau qui vient s'unir à un autre envoyé par has toujours (fig. 10) et on a tiré parti de cette disposition pour la classification, comme on jeut le voir dans le courant de l'ouyrage. Quant aux espaces qui sont entre les nervules, ce ne sont point it proprement parler des cellules, et on Jes appelle cspaces internervuraux. Ia troisième nervure (h) n'a point recu de nom bien précis, à cause de son peu d'importance; cn effet .lle ne se subdivise pas, et longe parallilement le bord interne. On yeut la nommer radiale ou sous-médiane. Indépendamment de ces nerrures, on peut voir dans la firure o un très-petit rameau sous la dernière; mais il n'existe que dans le genre Papilin.

La seconde aile (B) a reçu les mêmes noms quant aux angles et aux bords, avec les différences suivantes : a-d se nomme plus généraLa sonplle toujours dans notre ouyrage bord abdominal; d est un angle peu important et f'a pas d'autre nom que celui d'angle supérieur; e, au contraire, s'appelle exclusirement angle anal.

Guant aux nervures, leur disposition varie aussi un peu; leur direction est moins compliquée, la costale est moins importante et celles qui partent de la base de l'aile sont en plus grand nombre; mais elles n'ont point recu de noms particuliers. On retrouve ici la rellule, et c'est - lle qui fournit le meilleur caractère : le rameau qui la ferme est très-prononcé; et si clle paraît quelquefois fermée par une légére membrane, on n'en doit pas tenir compte et la considérer comme ouverte.

11 nous reste à parler des modifications que subissent les hords marginal et abdominal. Ces modifications pour le premier sont innombraHes : tintôt il est denlé régulierement (Argynnis); tantôt, sans présenter de dents distinctes, il cst coupé: pour ainsi dire à ficettes : c'est ce que nous appelons p.lygoné; tantôt il est composé d'angles inégraux (Vanessa); tantôt il est fulqué (Gonoptcryx; tantôt il est entier, et alors il Pire droil (Papilio) ou courbe (Polyommalus); ce qui fait appeler l'aile obtuse, arrondie, coupée carrément, etc.. etc. Aux inférieures, il - muni, dans quelanes espéces, d'un long appendice en forme de queue (Papilio), ou d'un filet léger et fragile (Poly. Boeticus); enfin il 'st mimi, dans que

Le bord ablominal de son côté offre plusicurs modifications; ainsi, il est ou échancré et plan, et alors il supprime une des nervures des

fan sans échancrí, ou plus ou moins corcare, et formant alors une sorte de gouttiere qui embrasse le dessous de l'abdomen:

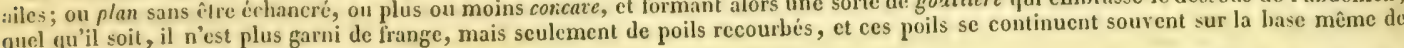
l'aile.

Telles sont les différentes parties des ailes. Nous allons roir maintenant en quoi consistent les dessins qui les ornent; et qui, quelque variés qu'ils soicnt, suivent cependant des règles générales.

\section{$\mathbb{S} \mathbf{V} . D e s$ dessins des ailes.}

Nous ayons indiqué dans le parnozraphe précídent les noms que les dessins tirent de leur position. Nous allons ajouter quelques spéciflcations i cette richle, et nous verrons ensuite quels sont les noms des dessins généraux, quelque position qu'ils occupent.

Conme nous l'ivons dit, toute tache partant de la côte est nomméc costale; si cette tache se prolonge au-deli du tiers de l'aile, elle devient une bande qu'on nommera également costale (e. fig. 1); mais si elle se prolonge plus avant, elle se nomme simplement bande transterse.

On donne, par extension, le nom de costale ì cette petite tache noire que les Pieris portent au bout de la cellule (a. fig. 12 ), bien que 
rhez la plupant d'entre elles ectte tache n'atteigne pass la côte. Par lit même fietiun, en womme apicale une tache qui est seulement dans lit région apicate, sans toucher l'angle de ce nom (b. fig. 12, et g. fig. 14).

Les anteurs ont jusqu’ici nommé égalenent dessin terminal ou marginal hut dessin qui s'approche seulement lu bord de ce nom: mais ces dessius sont d'ordinaire si compliqués, que nous n'avous pas cru devoir employer ce terme si vaguement. Nous n'appelons donc terminal ou marginal qu'un dessin qui touche immidiatement le hord. Tout antre qui en approche seulement ou le longe, est pour nous ante-terminal.

Nous croyons inutile d'expliquer ce que nous entendons par ligne intra-cellulaire (f. fig. 14), et de répieter la note de la page $7^{5}$ sur la tache annulaire (e. fir. 14) dans les satyres de la division 101 ; mais nous devons indiquer sur la pliache les lignes du dessous des inféricu-

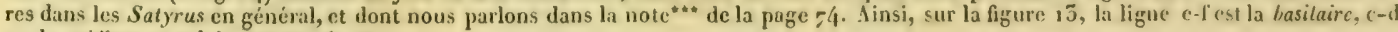
est la midiane et a-b l'anté-terminale.

On nomme point un dessin ordinairement arrondi et qui occupe peu d'espace (cependant rous donnons ígalement re uons it tout ait nen pupillé). Si ce destin aurmente de grandeur, il devient une tuche; si cette tache a une forme allongée, elle s'appelle bande; si au contraire la surface du dessin est très-petite relativement a sa longueur, il se nommera ligne s'il est très-long; trait s'il l'est moins; stric s'il est très-mienu ct trés-court.

Une ligne on bande est longitudinale si elle est parallèle aux nervures; transverse si clle les croise à peu prè à angle droit; oblique dan: les autres cas (ces termes ne doivent point s'entendre avec une rigueur mathématique). Nous pensons qu'il est inutile de difinir les lignes denties, frstonnées, courlies, arquées, bifides, etc., etc., non plus ujue les taches sagitties, cordiformes, pyriformes, etc., tous ces mot: portant leur étymologie arec eux.

Mais la forme oculie est si commune aux taches, que nous devons en indiquer les parties, qui sont souvent confondues par les amateurs et même par quelques écrivains.

Il suffit qu'une tache soit arrondic et porte au milieu un point de couleur différente pour recevoir le nom d'ceil. Ice point s'appelle alors pupille (d. tigr. 15 ), le cercle qui l'entoure prunelle (c. même fig.); enfin, si la prunelle est elle-même entourée d'un cercle nouven. celui-ci se nomme iris (b.fig. 15); passé ce nombre, les autres dessins entourants s'ippellent simplement des cercles (a. fig. 15, etc.).

On entend généralement par lunule toute tache en forme de croissant; cependant ce terme en entomologic est un de ceux qui reẹoirent le plus d'extention. Il faudra en tenir compte.

Enfin, une règle à peu près générale est celle-ci : les dessins aulres que les bandes transecrses ne sont point coupés par les nervurcs, ct occupent les espaces internervuraux. Cette observation, trés-utile pour la peinture des Lépidoptires, peut aussi être de quelque utilité pour lenr étude. 


\section{VOCABULAIRE.}

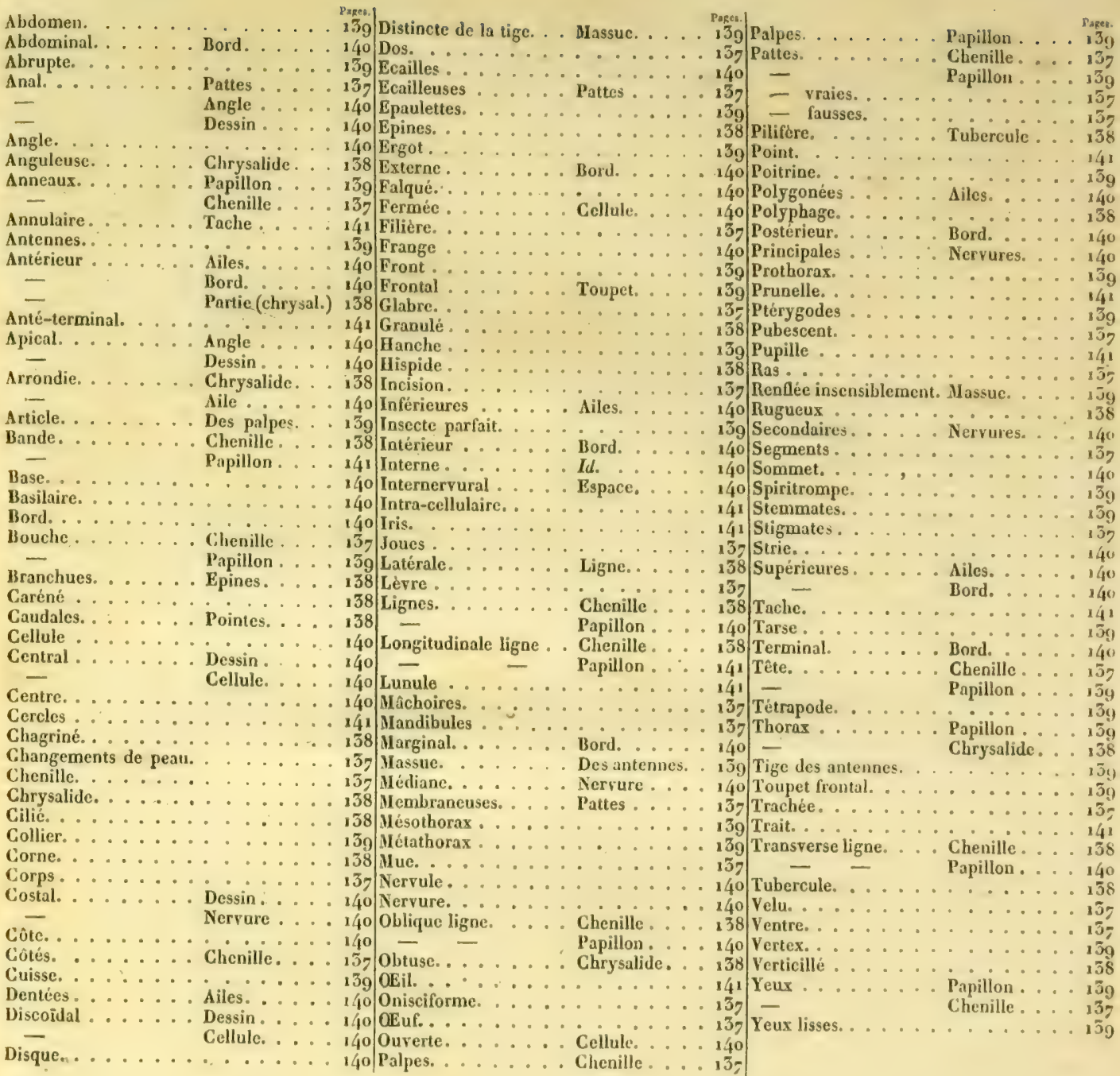


. 


\title{
ALPII ABÉTIQUE ET SYNONYMIQUE
}

\author{
DU PREMIER FOLUME.
}

Nola. Les noms des tribus sont en grandes majuscules, ceux des genres en petites majuscules, ceux des espèces adoptés dans cet ourrage en caracteres ordinaires, ceux de la synonymie en italique.

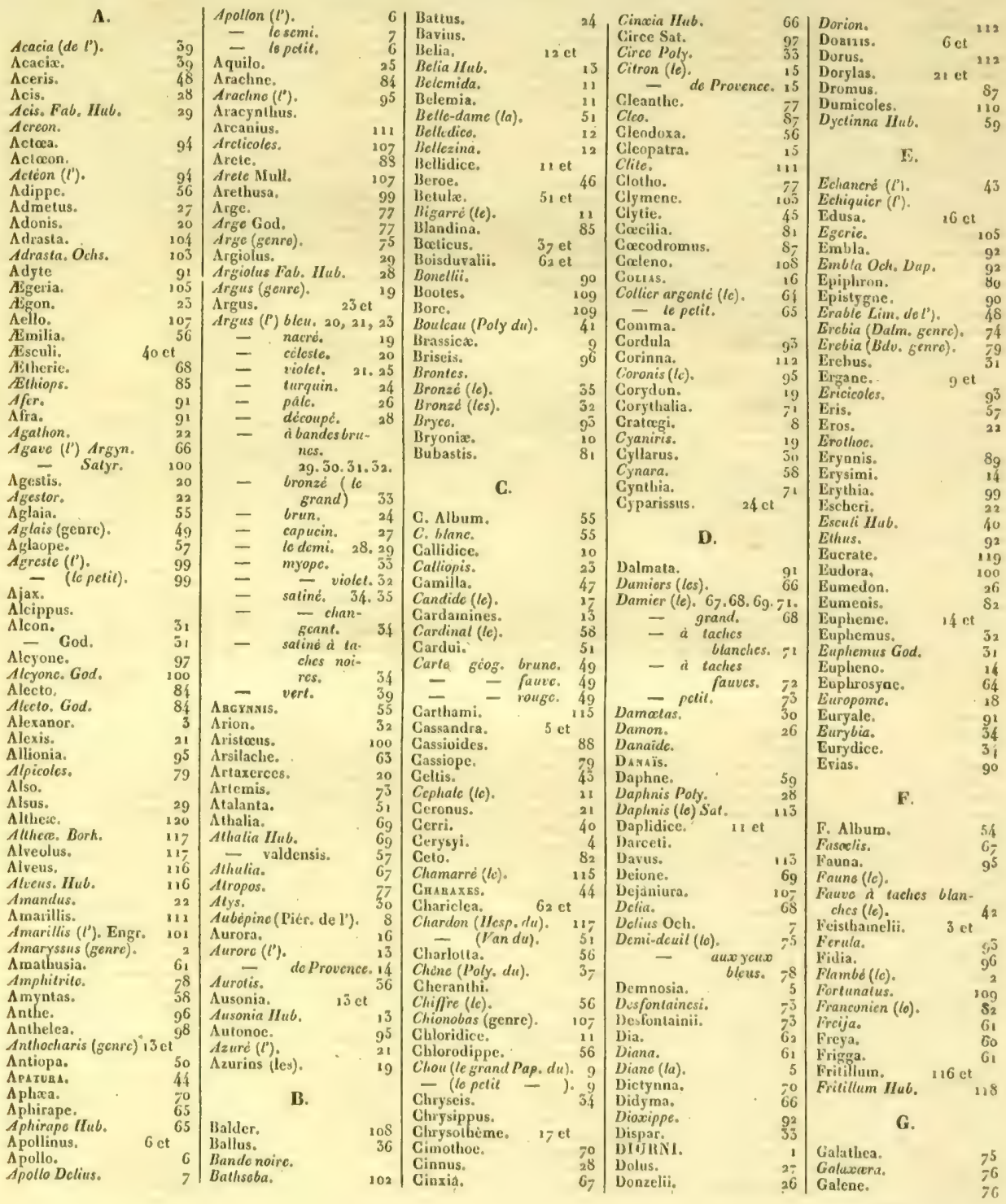




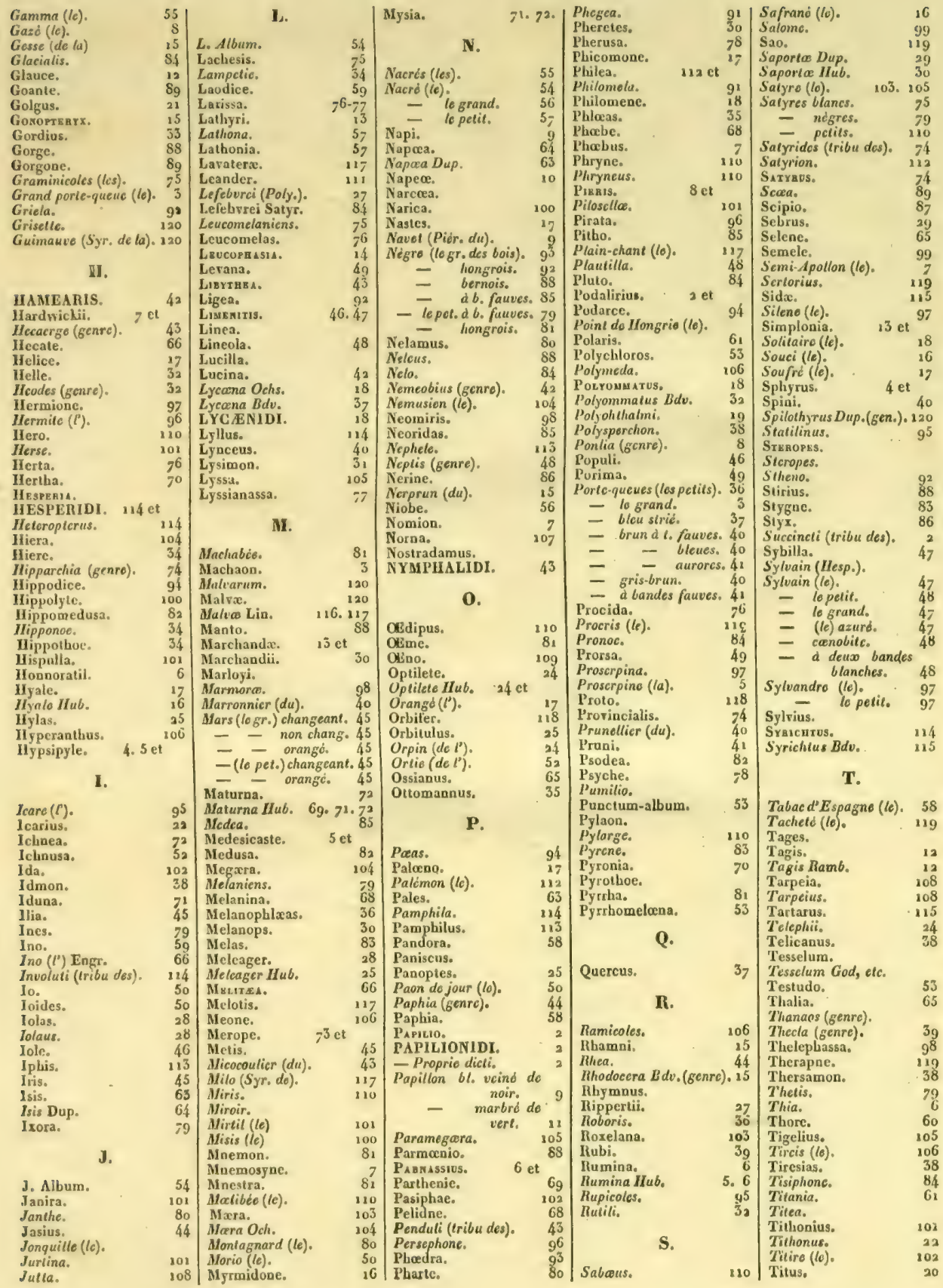


TABLE ALPHABÉTIQUE ET SYNONYMIQUE.

Tortue (la grande). - la moyenne.

- la petite.

Tremulae,

Triangulum.

Triclaris.

Tristan (le)

Tullia.

Tyndarus.

\begin{tabular}{r|l|l} 
& \\
53 & & \\
53 & \\
53 & Unedonis. \\
44 & Urticx. \\
47 & \\
65 & \\
106 & \\
113 & V. Album. \\
87 & V. blane.
\end{tabular}

U.

\begin{tabular}{l|l} 
& Valaision (te). \\
44 & Valesina. \\
52 & Vaxissa. \\
Venula. \\
Vertumne (le). \\
& Vicicoles. \\
54 & Violette(la petite). \\
54 & Virgaureæ. la grande.
\end{tabular}

\begin{tabular}{r|l}
58 & Vulcain (le). \\
58 & \\
49 & \\
16 & W. Album. \\
103 & W. Blanc. \\
62 & \\
59 & \\
35 & Xanthe.
\end{tabular}

5.)

Xanthe Nub. Xanthochloros.

Xanthomelas.

Xiphia.

Xuthus.

X.

33 Zephyrus (genre).

\section{ERRATA DU TOME PREMIER.}

Pag. 4, ligne 19. Chenille chargèo d'ejpines, charnue et velue. Lisez: Chenille chargée d'épines charnues et velues.

Idem. 3" colonne, ligne dernière, Chrysalide anguleuse. Lisez : Chrysalide peu anguleuse.

Pag. 11, 4* colonne, ligne 37. Bellidice. Lisez : Daplidice.

Pag. $12,4^{\circ}$ colonne, ligne 53 . Belledice. Lisez : Bellidice.

Pag. 38, $x^{\text {re }}$ colonne. Relevez les mots IDMON, AMYNTAS et riagsias, de sorte qu'ils se trouvent en face : le premier de la ligne15, le second de la ligne 33, et le troisième de la ligne 46 de la seconde colonne, aveo leur synonymie.

Pag. $48,1^{\text {re }}$ colonne, ligne 15. Platuilla. Lisez: Plautilla.

Pag. 55. Genre XIV (Argynnis), Lisez : Genre XV.

Pag. 6o, $2^{\text {xe }}$ colonne, ligue 16. Ereya, Lisez: Freija Idem.

Pag. 63, $4^{\circ}$ colonne, ligne $1^{r e}$. Touchant. Lisez; Tranchant.

Pag. 97, $4^{\circ}$ colonne, ligne 39. Fascelis Fab. au pas de Suze, Lisez: Fascelis Fab. pris au pas de Suze,

ligne dernière. Dans les bois. Lisez: Dans tous les bois.

Pag. 72, 4e colonne, ligne 18. La dernière série. Lisez: Quelquefois, la dernière série.

Pag. 74. Genre XVI (Satyrus). Lisez : XVII.

Idem, ligne 32. (118), Lisez: (121). 

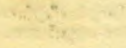


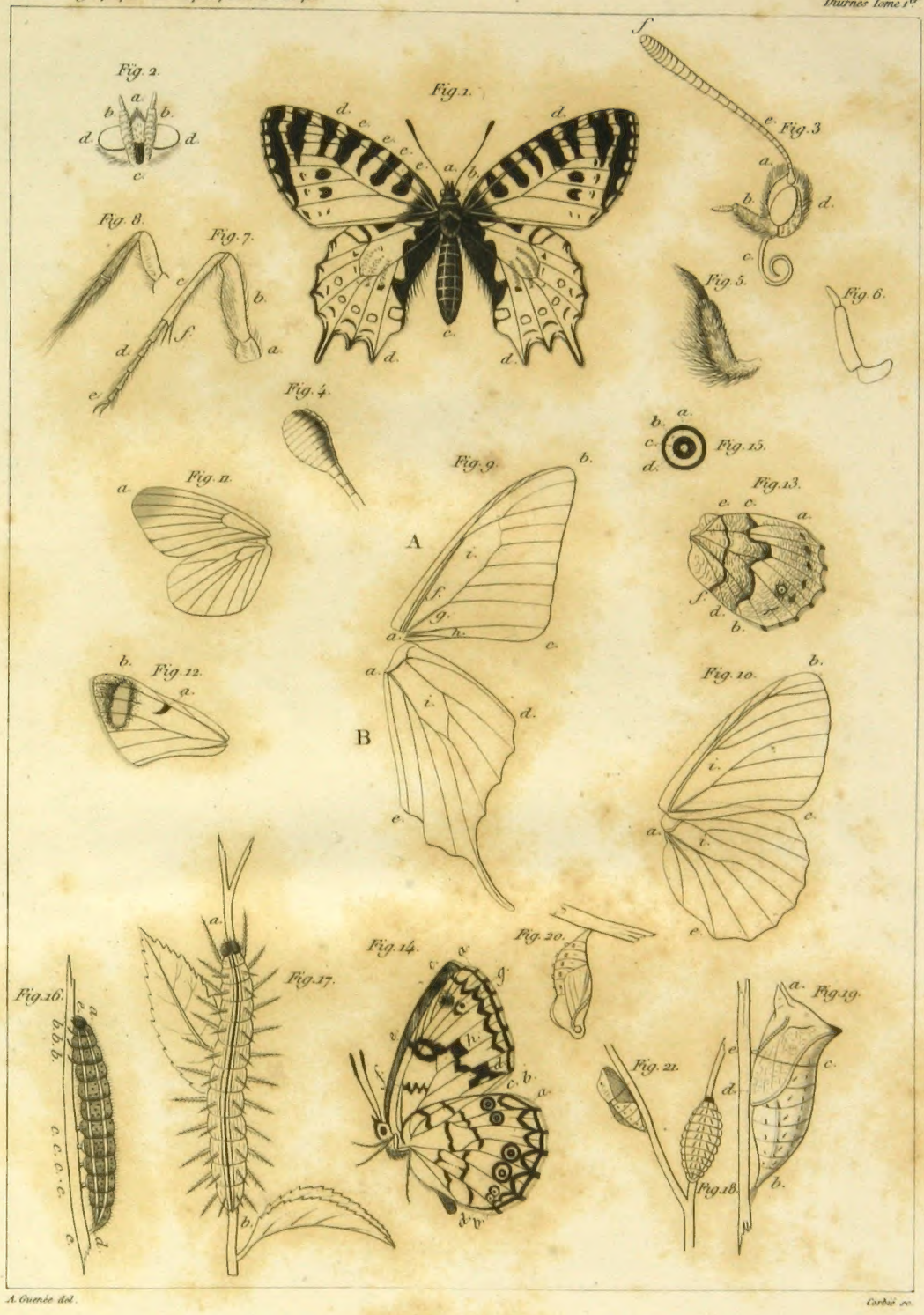

Planche explicative 
